\title{
Search for a low-energy excess of electron neutrinos in MicroBooNE
}

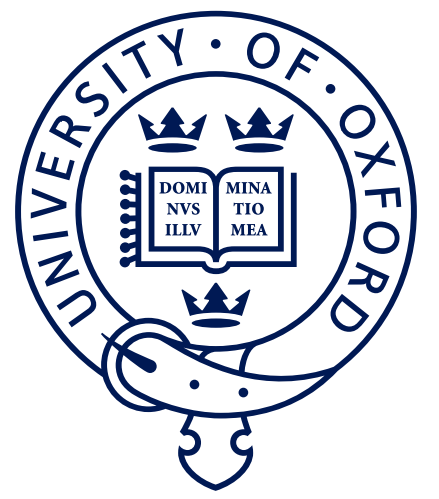

Stefano Roberto Soleti

St Catherine's College

University of Oxford

A thesis submitted for the degree of

Doctor of Philosophy

Hilary 2019 


\section{Statement of Originality}

This is to certify that to the best of my knowledge, the content of this thesis is my own work. This thesis has not been submitted for any degree or other purposes. I certify that the intellectual content of this thesis is the product of my own work and that all the assistance received in preparing this thesis and sources have been acknowledged. Results and figures from published works by others have been clearly attributed.

Chapters 1 and 2 contain a theoretical introduction to the state of the art in the field. Chapter 3 shows the previous results on the same topic and the motivations of the study presented here. Chapter 4 gives a brief overview of the MicroBooNE detector, whose data was used to produce the results of this thesis. Chapter 5 describes the reconstruction techniques used to allow a high-level analysis of the data. The analysis is thoroughly described in Chapters 6, 7, 8, and, where not specified otherwise, is entirely my own work.

I am the corresponding author of the paper in Appendix A, published in Journal of Instrumentation. I produced all the results and plots shown there. 


\section{Acknowledgements}

Three (and a half) years looked like a long time when I started my DPhil at Oxford. However, thank to a large number of people, this period passed in the blink of an eye: time flies when you're having fun!

First of all I would like to thank my advisor Roxanne Guenette. You have been not only an excellent mentor but also an extremely supportive, helpful and friendly person. I am very grateful for the countless quick chats we had about physics, work, and life. Few lines are not enough to thank you for the opportunities you gave me during the DPhil and the once-in-a-lifetime chance to work with you at Harvard. I have been very fortunate. I would also like to thank my advisor Alfons Weber for assisting me in Oxford and helping me with the several postdoc applications!

I also had the luck to work with great colleagues, who gave me advice throughout these years and helped me to produce the results shown in this thesis. Thank you Marco, Wouter, and Nicolò. A special mention goes of course to Corey, Matt, and Justo, the best postdocs a graduate student can ask for.

Being part of MicroBooNE, I also had the opportunity to work with some of the most talented physicists in the world. The special environment offered by Fermilab allowed me to grow as a physicist and as a person.

My time in Oxford wouldn't have been the same without the great friends I made here. Thanks to Andrea, Giovanna, Angela, Martin, Mattia, Virginia and many others for the innumerable dinners at CS. Also my period in Boston felt like home, thanks to Cinzia, Carmela, Giorgio, Steven and the countless number of "Italians" I met there.

It is not common to keep friends in your hometown when you are there a couple of weeks per year. However, Vito, Valerio, Mattia, Leonardo, and Francesco were always ready to have fun whenever I was there.

Even if far, my mother and my father were always very supportive of all my choices and ready to give me a warm welcome back every time I was at home. I wouldn't (literally) be here if it wasn't for them.

Lastly, but most importantly, I would like to thank my partner Marina, the most supportive, amazing, and wonderful person I have in my life. Meeting you was the best (and luckiest) thing of these years in Oxford. 


\section{Abstract}

The Micro Booster Neutrino Experiment (MicroBooNE) is a Liquid Argon Time Projection Chamber (LArTPC) designed for short-baseline neutrino physics at the Fermi National Accelerator Laboratory. The main physics goal of MicroBooNE is to address the low-energy excess of electron-like events observed by the MiniBooNE experiment and, if confirmed, clarify its nature. The MiniBooNE experiment is a Cherenkov detector and this technology does not allow to distinguish between electrons and single photons in the final state. LArTPC detectors, instead, offer excellent granularity and powerful separation between electrons and photons. For this reason, they represent an ideal technology for the detection of electron neutrino interactions. This thesis presents the first fully-automated electron neutrino selection in a LArTPC. The selection looks for charged-current electron neutrino interactions with no pions and at least one proton in the final state. It is applied on a sub-sample of the data acquired by the detector in the Booster Neutrino Beam, corresponding to $4.34 \times 10^{19}$ protons-on-target. A validation of the analysis is performed on two orthogonal side-bands, enriched with neutral-current and charged-current muon neutrino interactions, respectively. The uncertainties on the neutrino cross sections, flux, and detector simulation are evaluated. The MicroBooNE detector is placed off-axis with the Neutrinos at the Main Injector (NuMI) beam. An independent dataset of events acquired by triggering on the NuMI beam is employed to measure the significance of the detection of electron neutrinos in the beam using the selection presented here. The sensitivity of the MicroBooNE experiment to the MiniBooNE low-energy excess of electron-like events is evaluated. The efficiency and backgroundrejection power necessary to achieve $5 \sigma$ sensitivity are also quantified. 


\section{Contents}

List of Figures viii

List of Tables $\quad$ xx

List of Abbreviations $\quad$ xxi

1 Introduction 1

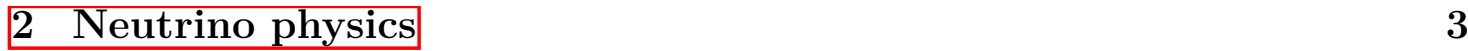

2.1 Introduction . . . . . . . . . . . . . . . . . . . 3

2.2 Neutrino Oscillations Theory . . . . . . . . . . . . . . . . . 5

$2.3 \quad$ Experimental Evidence of Neutrino Oscillation . . . . . . . . . . . . 8

2.4 Massive neutrinos in the Standard Model . . . . . . . . . . . . . . . 13

2.4 .1 The seesaw mechanism . . . . . . . . . . . . . . 15

2.5 Neutrino interaction modes . . . . . . . . . . . . . . . 16

2.6 Future research efforts . . . . . . . . . . . . . . . . . . . . . . . . 19

3 The LSND and MiniBooNE anomalies 21

3.1 The LSND experiment . . . . . . . . . . . . . . . . . . . . . . 21

3.2 The MiniBooNE experiment . . . . . . . . . . . . . . . . . . . . . . 24

3.3 Oscillations anomalies: the global picture . . . . . . . . . . . . . . . 29

3.3 .1 Radiochemical experiments . . . . . . . . . . . . . . 30

3.3 .2 Reactor experiments . . . . . . . . . . . . . . . . . . . . . . . . . . . . . . 33

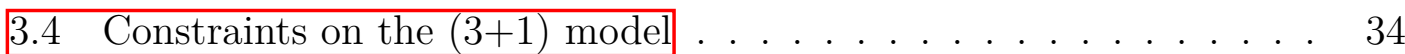

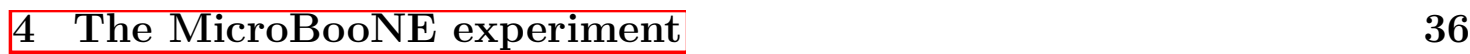

4.1 Motivation . . . . . . . . . . . . . . . . . . . 37

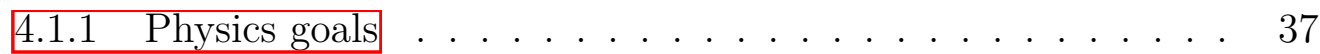

4.1 .2 Research and development goals . . . . . . . . . . . . . . 39

4.2 The LArTPC detection technology . . . . . . . . . . . . . . . . . . 40

4.3 The neutrino beams at Fermilab . . . . . . . . . . . . . . . . . 45

4.3 .1 The Booster Neutrino Beam . . . . . . . . . . . . . . . 46

4.3 .2 Neutrino flux simulation . . . . . . . . . . . . . . . 47 
4.4 The MicroBooNE detector . . . . . . . . . . . . . . . . . . . . . . 49

4.4 .1 The Time Projection Chamber . . . . . . . . . . . . . . . . 49

$4.4 .2 \quad$ The Light Collection system . . . . . . . . . . . . . . . . . 51

4.4 .3 Cryogenics and purification . . . . . . . . . . . . 52

4.4 .4 Electronics and readout . . . . . . . . . . . . . . . . . 53

4.4.5 The MuCS and the Cosmic-Ray Tagger . . . . . . . . . . . . 54

4.4 .6 Trigger system . . . . . . . . . . . . . . . 55

5 Event reconstruction $\quad 58$

5.1 Signal processing . . . . . . . . . . . . . . . . . . 58

5.1 .1 Noise removal . . . . . . . . . . . . . . . . . . . . . . . . . 58

$5.1 .2 \quad$ Signal deconvolution . . . . . . . . . . . . . . . . . 59

5.13 Hit reconstruction . . . . . . . . . . . . . . . . . . . . . . . 61

5.2 The Pandora multi-algorithm pattern recognition . . . . . . . . . 61

6 Selection of electron neutrinos in the MicroBooNE experiment 64

6.1 Signal definition . . . . . . . . . . . . . . . . 65

6.2 Analysis Methodology . . . . . . . . . . . . . . . . 67

$6.2 .1 \quad$ Data and Monte Carlo samples . . . . . . . . . . . . . 68

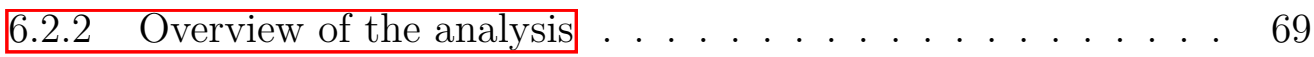

6.2 .3 Cosmic-ray rejection . . . . . . . . . . . . . . . . 71

6.2 .4 Optical selection . . . . . . . . . . . . . . . . . 73

6.2 .5 Topological pre-selection . . . . . . . . . . . . . . . . 77

6.2 .6 Minimum reconstruction quality requirements . . . . . . . . 78

6.2 .7 Selection efficiency and purity . . . . . . . . . . . . . . . . . 79

6.2 .8 Selection performances in BNB events . . . . . . . . . . . 84

6.3 Calorimetry . . . . . . . . . . . . . . . . . . . 88

$6.3 .1 \quad$ Scope of the energy reconstruction . . . . . . . . . . 88

6.3 .2 Electron energy reconstruction and calibration . . . . . . . . 90

6.3.3 Single proton energy reconstruction and calibration . . . . . 94

6.3 .4 Deposited Energy Reconstruction . . . . . . . . . . . . . . . . . . 96

6.3 .5 Deposited energy binning . . . . . . . . . . . . . . . . . 98

6.3 .6 Measurement of the electromagnetic shower energy loss . . . 99

6.3.7 Particle identification of reconstructed tracks . . . . . . . . . 103

6.4 Background Rejection . . . . . . . . . . . . . . . . 106

6.4 .1 Rectangular cuts . . . . . . . . . . . . . . . 106

6.4 .2 Boosted Decision Trees . . . . . . . . . . . . . . . . . . . . . 120

6.4 .3 Interaction types . . . . . . . . . . . . . . . . . . . 128

6.5 Validation . . . . . . . . . . . . . . . . . . . . 128

6.5 .1 Electromagnetic shower energy loss . . . . . . . . . . . . . . 128

6.5 .2 Side-bands checks . . . . . . . . . . . . . . . . . . . . . 129

6.5 .3 NuMI beam event studies . . . . . . . . . . . . . . . . . . 131 
\begin{tabular}{lll}
\hline Systematic uncertainties & 135
\end{tabular}

7.1 Introduction . . . . . . . . . . . . . . . . . . . . . . . 135

7.2 Flux systematic uncertainties . . . . . . . . . . . . . . . 137

7.3 Cross-section systematic uncertainties . . . . . . . . . . . . . . . . . 140

7.4 Detector systematic uncertainties . . . . . . . . . . . . . . . . . . . 140

7.5 Summary . . . . . . . . . . . . . . . . . . . . 145

8 Sensitivity to the MiniBooNE low-energy excess 148

8.1 Estimation of the MiniBooNE signal in MicroBooNE . . . . . . . . 148

8.2 Sensitivity to the excess $\ldots \ldots \ldots \ldots$. . . . . . . . . . . . 151

8.3 Future improvements . . . . . . . . . . . . . . . . . . . . . 154

8.4 Improvements and sensitivity . . . . . . . . . . . . . . . . 155

\begin{tabular}{llr}
\hline & Conclusions & 157
\end{tabular}

\section{Appendices}

A Measurement of cosmic-ray reconstruction efficiencies in the MicroBooNE LArTPC using a small external cosmic-ray counter 161

\begin{tabular}{lr}
\hline References & 181
\end{tabular} 


\section{List of Figures}

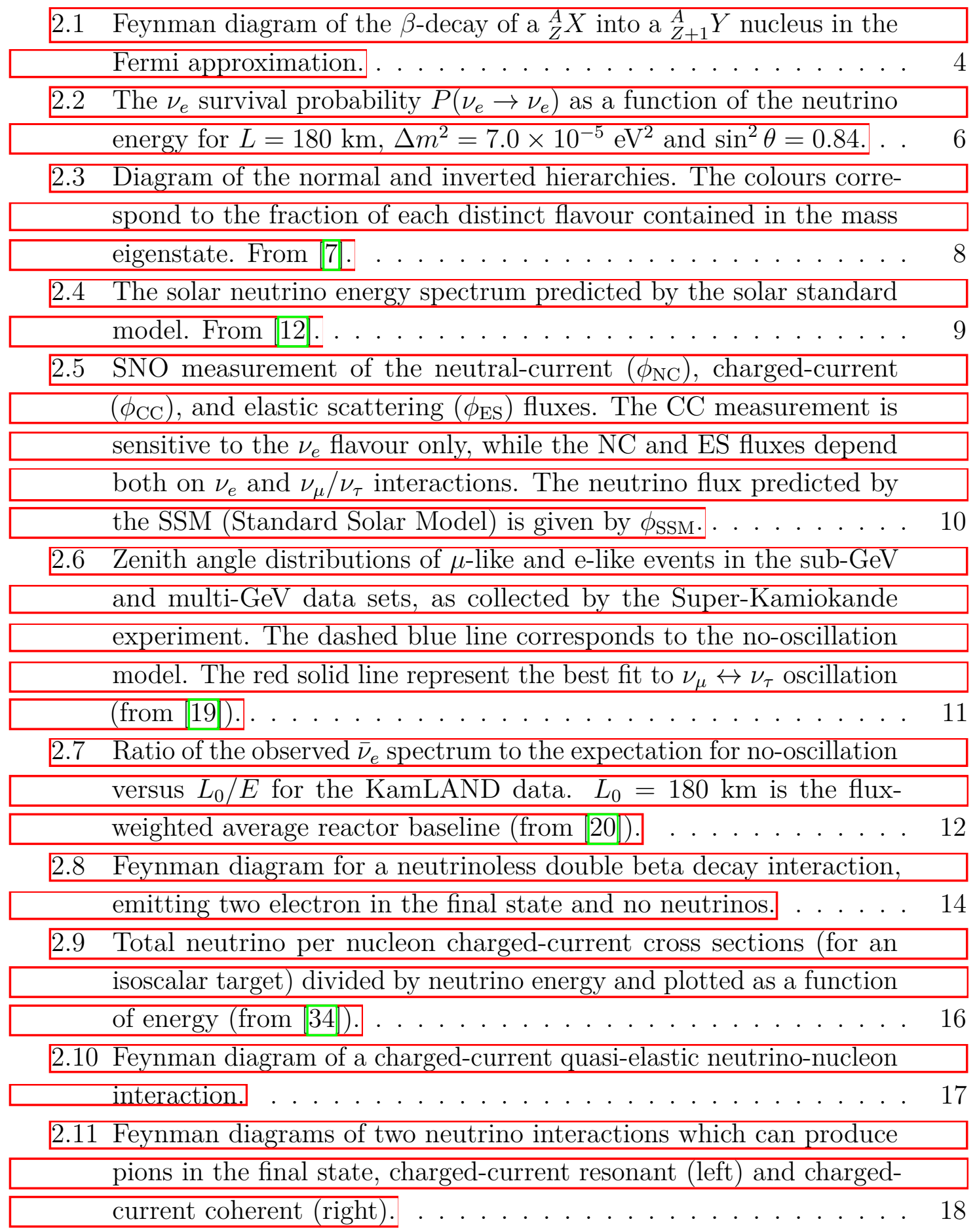


2.12 Feynman diagram of a charged-current deep inelastic scattering - neutrino-nucleon interaction. . . . . . . . . . . . . . . . . . 19

3.1 A schematic of the LSND experiment and its detection technique: the inverse $\beta$-decay of the neutrinos in the detector produce Cherenkov and scintillation light, in delayed coincidence with the light emitted by the neutron capture. . . . . . . . . . . . . . . . . 22

3.2 The excess of electron antineutrinos observed by the LSND experiment (left) can be interpreted with the presence of a fourth neutrino state. The mixing angles and mass splittings allowed by the LSND data are shown on the right at 90\% C.L (blue) and 99\% C.L. (yellow), together with the $90 \%$ C.L. exclusion limits from other experiments (solid red lines). From 43]. . . . . . . . . . . . . . . 23

3.3 Schematics and event display for three topologies in the MiniBooNE

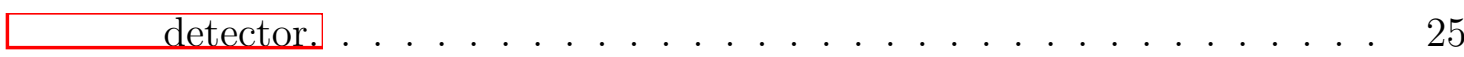

3.4 The MiniBooNE neutrino mode corresponding to the total $12.84 \times 10^{20}$ POT data, for $\nu_{e}$ CCQE data (points with statistical errors) and background (histogram with systematic errors). The dashed line represent the two-neutrino model best fit (left). The appearance probability as a function of the $L / E$ ratio is in agreement with LSND data (right). Adapted from 48. . . . . . . . . . . . . . 26

3.5 Neutrino mass normal hierarchy in the scenario of $3+1$ neutrinos (from 6[). . . . . . . . . . . . . . . . . 29

3.6 MiniBooNE allowed regions for the combined neutrino mode and \begin{tabular}{|c|c|c|}
\hline antineutrino for events with $200<E_{\nu}^{Q E}<3000 \mathrm{MeV}$ within a two- \\
\hline
\end{tabular} neutrino oscillation model. The black point at $\left(\sin ^{2} 2 \theta, \Delta m^{2}\right)=$ $\left(0.96,0.041 \mathrm{eV}^{2}\right)$ represents the best fit $48 . \ldots . . . . . . .30$

3.7 The squared-mass splittings and mixing angles favoured (solid regions) or excluded (open regions) by existing neutrino oscillation measurements. Results are categorised by channels: $\nu_{e}$ disappearance (solid lines), $\nu_{\mu} \leftrightarrow \nu_{\tau}$ (dotted lines), $\nu_{e} \leftrightarrow \nu_{\tau}$ (dashed lines), and $\nu_{e} \leftrightarrow \nu_{\mu}$ (dashed-dotted lines). The normal mass ordering is assumed where relevant. Taken from 19 . Does not include MiniBooNE latest result $48 \ldots \ldots \ldots \ldots \ldots \ldots \ldots$

3.8 The SAGE and GALLEX experiments observed a deficit of electron \begin{tabular}{|c|}
\hline neutrino interactions using radioactive isotopes, which could be \\
\hline
\end{tabular} explained by introducing oscillations into a sterile neutrino state. . . 32

3.9 Fraction between observed and predicted $\bar{\nu}_{e}$ flux at several reactor neutrino experiments. Several models for sterile neutrino oscillation for different mass splitting terms and mixing angles are also shown. 33 
3.10 Inverse $\beta$-decay yield per fission, $\sigma_{f}$, versus effective ${ }^{239} \mathrm{Pu}$ (lower axis) or ${ }^{235} \mathrm{U}$ (upper axis) fission fraction. From $|55| . \quad$. . . . . . . . 34

3.11 There is a severe tension between appearance and disappearance \begin{tabular}{|l|}
\hline results within the $(3+1)$ model. The free (fixed) fluxes lines of the \\
\hline \hline plots on the left refer to the constraining (or not) of the reactor fluxes \\
\hline \hline in the fits. Adapted from 62 . . . . . . . . . . . . . . . . 35
\end{tabular}

4.1 Diagram of the operating principle of the MicroBooNE LArTPC, showing the waveforms produced by the ionisation trails in the collection plane (red) and in one induction plane (blue). . . . . . . . 41

4.2 The ICARUS collaboration measured the recombination factor $R$, \begin{tabular}{|c|}
\hline defined as the ratio between the measured and the theoretical stopping \\
\hline power, as a function of the $d E / d x$ (left) and of the electric field (right). \\
\hline
\end{tabular} power, as a function of the $d E / d x$ (left) and of the electric field (right). From 80 . . . . . . . . . . . . . . . . . . 4 43

$4.3 \quad$ Predicted $\left(\Delta y^{\mathrm{MC}}\right)$ and measured $\left(\Delta y^{\text {Data }}\right)$ space-charge distortions \begin{tabular}{|c|}
\hline as a function of the drift coordinate at both the top and bottom of \\
\hline
\end{tabular} \begin{tabular}{|c|}
\hline the TPC. The discrepancy between data and Monte Carlo is caused \\
\hline
\end{tabular} by the absence of the liquid argon flow in the simulation. Error bars are statistical only. From 83 . . . . . . . . . . . . . . . . . . 44

4.4 Variation of the $Q_{A} / Q_{C}$ charge ratio between February and April

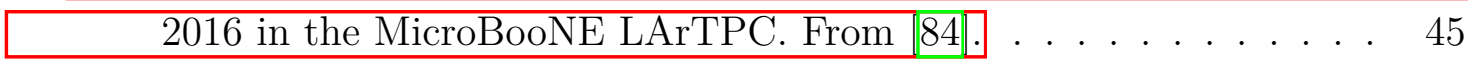

4.5 Schematic of the Booster Neutrino Beam chain in neutrino mode, showing, from left to right, the focusing horn, the decay pipe, the

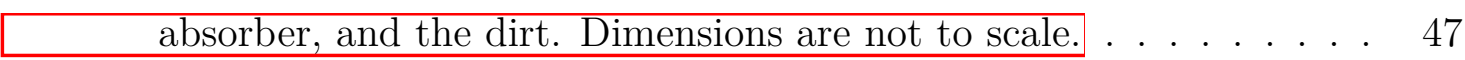

$4.6 \quad$ BNB absolute flux prediction in neutrino mode at the MicroBooNE

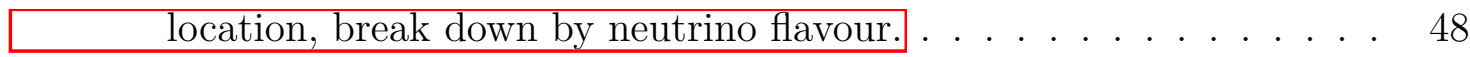

4.7 3D rendering of the MicroBooNE cryostat, showing the TPC wire \begin{tabular}{|l|}
\hline cage and the feedthroughs, which take the signals from the wires and \\
\hline the PMTs (not shown) to the DAQ. In this rendering, the cathode is
\end{tabular} \begin{tabular}{|c|}
\hline the PMTs (not shown) to the DAQ. In this rendering, the cathode is \\
\hline on the right and the wire planes on the anode side are not shown. . 50
\end{tabular}

4.8 Drawing of the MicroBooNE TPC showing the coordinate system \begin{tabular}{|c|}
\hline and the size of each side. The anode and the cathode are respectively \\
\hline
\end{tabular} on the right and the left, as seen from the beam. . . . . . . . . . . . 51

4.9 Event display of a $\nu_{\mu} \mathrm{CC} \pi^{0}$ candidate in the collection plane. The \begin{tabular}{|c|}
\hline colour scale corresponds to the amount of charged deposited on the \\
\hline
\end{tabular}

\begin{tabular}{|l|}
\hline wires. . . . . . . . . . . . . . . . . . . . . . . . . . . . . 52 \\
\hline 4.10 Scintillation light emission spectrum (red) and TPB re-emission \\
\hline spectrum (green), with the quantum efficiency of the PMTs employed \\
\hline \hline in MicroBooNE (blue). PMT borosilicate glass transmittance is \\
\hline represented by the solid black line. From 67 . . . . . . . . 52
\end{tabular}


4.11 3D drawing of simulated cosmic rays (brown lines) hitting the

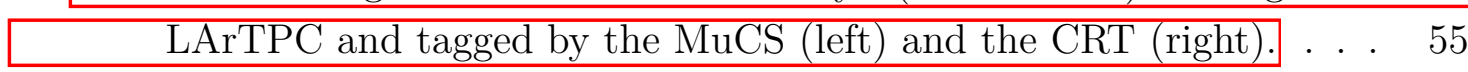

4.12 Software trigger efficiency as a function of the PE threshold, both

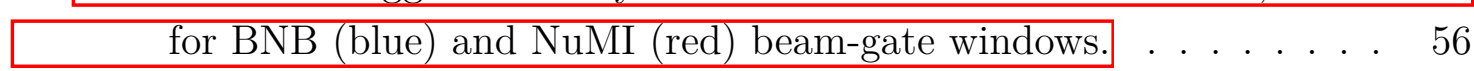

4.13 Distribution of optical flash times with respect to the trigger time for BNB triggered events, shown as a ratio to the expected cosmic \begin{tabular}{|rr}
\hline rate from beam-off data, collected with the EXT trigger. . . . . . . 57 & 57
\end{tabular}

5.1 Data event display of the $\mathrm{V}$ induction plane showing the raw signal \begin{tabular}{|c|}
\hline before (a) and after (b) offline noise filtering. The horizontal axis \\
\hline corresponds to the wire coordinate and the vertical axis correspond \\
\hline to the drift time. The colour scale represents the ADC counts from \\
\hline
\end{tabular} \begin{tabular}{|l|l|}
\hline to the drift time. The colour scale represents the ADC counts from \\
\hline the baseline in arbitrary units. From 94 . . . . . . . . . . . . . . . 60
\end{tabular}

5.2 Data event display with the waveform collected in a wire of the \begin{tabular}{|c|}
\hline collection plane. The white box corresponds to the waveform in the \\
\hline
\end{tabular}

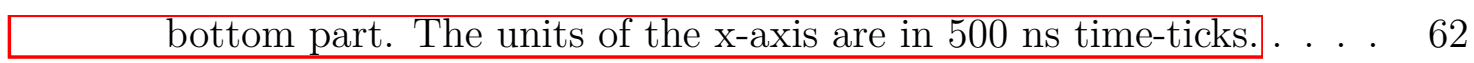

5.3 Pandora pattern recognition output of a simulated CC $\nu_{\mu}$ event with a muon (red), a proton (blue) and a charged pion (pink) in the final state. Each particle is reconstructed as a separate cluster and the positron coming from the $\pi^{+} \rightarrow \mu^{+} \rightarrow e^{+}$decay chain is classified as a daughter of the pion. From $97 . \ldots . \ldots . \ldots 63$

6.1 Monte Carlo $\nu_{e} \mathrm{CC} 0 \pi$-Np event display of the collection plane with an electron and two protons in the final state. The reconstructed shower-like object is represented by the green cone. The reconstructed track-like objects are represented by the red lines. The ionisation \begin{aligned} \hline trails without an associated reconstructed track are cosmic rays \\ \hline\end{aligned} correctly tagged by the cosmic-removal algorithms, described in \begin{tabular}{|r|r|r|}
\hline Section & 6.2 .3 & The colour scale is proportional to the amount of \\
\hline
\end{tabular} \begin{tabular}{|c|}
\hline charge collected by the wires. The vertical gaps are caused by the \\
\hline presence of unresponsive wires in the detector, which are turned off \\
\hline
\end{tabular} - in the simulation. . . . . . . . . . . . . . . . 67

6.2 Schematics of the $\nu_{e} \mathrm{CC} 0 \pi$-Np event selection stages, from the cosmic-ray removal to the rejection of the neutrino and cosmogenic -backgrounds. . . . . . . . . . . . . . . . . . . . 69

6.3 Event display of the collection plane with a muon stopping and \begin{tabular}{|c|}
\hline decaying, producing a Michel electron. Being low-energetic, it \\
\hline
\end{tabular}

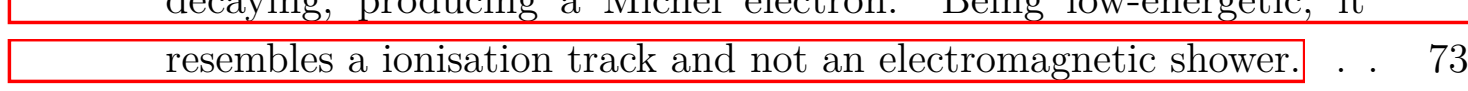

6.4 Reconstructed PE distribution for signal (left) and cosmic background - (right) events. . . . . . . . . . . . . . . . 74 
6.5 Distribution of the distance between the reconstructed flash position \begin{tabular}{|l|}
\hline and the centre of the deposited charge on the $z$ axis for signal (left) \\
\hline \hline and cosmic background (right). The within $5 \mathrm{~cm}$ and further than \\
\hline $5 \mathrm{~cm}$ categories refers to the distance between the reconstructed \\
\hline
\end{tabular}

neutrino vertex and the true neutrino vertex. . . . . . . . . . . . . 75

6.6 Distribution of the ratio between the charge in the collection plane

\begin{tabular}{|l|}
\hline associated to the neutrino candidate (in ADC counts) and the number \\
\hline of PEs collected by the PMTs. The within $5 \mathrm{~cm}$ and further than \\
\hline $5 \mathrm{~cm}$ categories refers to the distance between the reconstructed \\
\hline
\end{tabular}

6.7 An example of flash-matching. The event has 4 neutrino candidates and we make a flash hypothesis for each one, shown in red, purple,

\begin{tabular}{|l|}
\hline and we make a flash hypothesis for each one, shown in red, purple, \\
\hline green, and brown. The observed flash corresponds to the filled blue \\
\hline area. A minimum binned likelihood is calculated, varying the $x$ \\
\hline position of the interaction. The match score is the inverse of the \\
\hline likelihood. The candidate with the highest match score is chosen as \\
\hline neutrino interaction candidate (the green one in this case). . . . . . 77
\end{tabular}

6.8 Schematic of the fiducial volume used in this analysis. The solid line \begin{tabular}{|c|}
\hline corresponds to the TPC borders and the dashed red line corresponds \\
\hline
\end{tabular}

- to the fiducial volume borders. . . . . . . . . . . . . . . . . 78

$6.9 \quad \nu_{e} \mathrm{CC} 0 \pi$-Np selection efficiency on single particles as a function of

\begin{tabular}{|c|}
\hline the true electron (left) and proton (right) kinetic energy. The dashed \\
\hline
\end{tabular}
lines correspond to the threshold applied at truth level. . . . . . . . 79

6.10 Simulated $\nu_{e} \mathrm{CC} 0 \pi$-Np true neutrino energy spectrum in the $0-3 \mathrm{GeV}$ range. Each true proton (true electron) in the final state is required \begin{tabular}{|cc}
\hline to have a kinetic energy larger than $40 \mathrm{MeV}(30 \mathrm{MeV})$. \\
\hline
\end{tabular}

$6.11 \nu_{e} \mathrm{CC} 0 \pi$-Np selection efficiency as a function of the true $\nu_{e}$ energy. Each true proton (true electron) in the final state is required to have a kinetic energy larger than $40 \mathrm{MeV}(30 \mathrm{MeV})$. The inner error bars represent the Monte Carlo statistical uncertainty, while the outer error bars are obtained summing in quadrature the statistical and the systematic uncertainties. . . . . . . . . . . . . . . . 81

6.12 Stacked histogram of generated events as a function of the true neutrino energy, categorised into correctly identified signal events in grey (passed) and different reconstruction or identification failure modes in colour. . . . . . . . . . . . . . . . . . . . . . 83

6.13 Stacked histogram of generated events as a function of the electron $\theta$

\begin{tabular}{|c|}
\hline (left) and $\phi$ (right) angles, categorised into correctly identified signal \\
\hline \hline events (passed) in grey and different reconstruction or identification \\
\hline failure modes in colour.
\end{tabular}


6.14 Distributions of the track and shower multiplicities in data and Monte - Carlo simulation. . . . . . . . . . . . . . . 86

6.15 Distributions of the inclination angle $\theta$ and the azimuthal angle $\phi$ of the reconstructed showers in the selected events for each event category. The black points represent the data with statistical uncer-

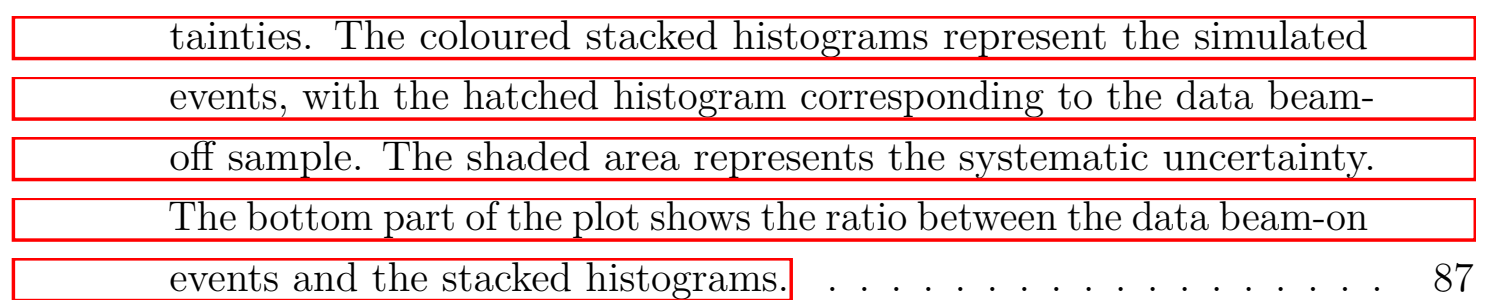

6.16 Distributions of the inclination angle $\theta$ and the azimuthal angle $\phi$ of the reconstructed showers, classified according to the primary particle that generated them. The black points represent the statistically subtraction of the data beam-off events from the data beam-on events.

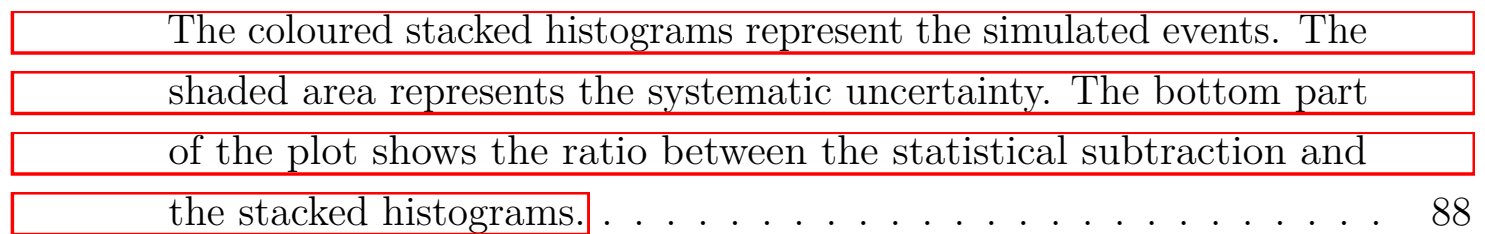

6.17 Event displays of the collection plane of three $\nu_{e}$-like data events \begin{tabular}{|l|}
\hline selected by our algorithm. The gaps are caused by the presence of \\
\hline dead or unresponsive wires. The red lines correspond to reconstructed \\
\hline track-like objects and the green cones correspond to reconstructed \\
\hline shower-like objects. … . . . . . . . . . . . . . . . . . . . . . .
\end{tabular}

6.18 Reconstructed (blue) and true (black) energy distribution for 10 \begin{tabular}{|l|}
\hline intervals of equal size in the $30-2030 \mathrm{MeV}$ energy range. The \\
\hline reconstructed energy distribution have been fitted with a GaussExp \\
\hline function (red line). . . . . . . . . . . . . . . . . . . . . . . . . . . 91
\end{tabular}

6.19 Bi-dimensional histogram of reconstructed electron energy $E_{\text {reco }}^{e}$ vs. \begin{tabular}{|l|}
\hline true electron deposited energy $E^{e}$. The reconstructed electron \\
\hline energy is measured summing the energy of each hit associated to \\
\hline reconstructed showers produced by the simulated electron. The black \\
\hline points correspond to the most probable value of the $E_{\text {reco }}^{e}$ distribution \\
\hline for each $E^{e}$ bin, calculated with a GaussExp fit, as illustrated in \\
\hline Figure 6.18, The red line represents a linear fit of the black points. \\
\hline 6.20 Normalised energy difference $E_{\text {frac }}$ for 10 intervals of equal size in \\
\hline the 30-2030 MeV energy range. The normalised energy difference \\
\hline distributions (blue) have been fitted with a GaussExp function (red \\
\hline line). . . . . . . . . . . . . . . . . . . . . . . . . . . . . . . . . . . . 9 93
\end{tabular} 
6.21 Fractional energy resolution for 10 intervals of equal size in the 30-

\begin{tabular}{|l|}
\hline $2030 \mathrm{MeV}$ energy range. The points, extracted from simulated events, \\
\hline have been fitted the classic calorimeter energy resolution equation \\
\hline \hline 6.9 (red line). . . . . . . . . . . . . . . . . . . 94
\end{tabular}

6.22 Proton kinetic energy as a function of the range of the proton in

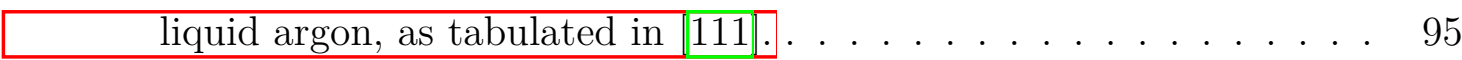

6.23 Bi-dimensional histogram of true proton energy $E^{p}$ vs. reconstructed proton energy $E_{\text {reco }}^{p}$. The calibration is calculated from a linear fit of

\begin{tabular}{|l|}
\hline the most probable values of the $E_{\text {reco }}^{p}$ distribution for each $E^{p}$ bin. \\
\hline The black points correspond to the most probable value of the $E_{\text {reco }}^{p}$ \\
\hline distribution for each $E^{p}$ bin, calculated with a GaussExp fit. The \\
\hline
\end{tabular}
red line represents a linear fit of the black points. . . . . . . . . . . 96

6.24 Bi-dimensional histogram of the total reconstructed energy $E_{\text {corr }}$ vs. \begin{tabular}{|c|c|c|}
\hline the total visible energy $E^{\mathrm{k}}$ vs. Black points are obtained measuring \\
\hline
\end{tabular} \begin{tabular}{|c|}
\hline the most probable value of the $E_{\text {corr }}$ distribution for each $E_{\mathrm{k}}$ bin, \\
\hline
\end{tabular} obtained with a GaussExp fit. . . . . . . . . . . . . . . . . . 97

6.25 Migration matrix between $E_{\text {deposited }}$ and $E_{\mathrm{k}}$. It shows the probability that an event with a true energy $E_{\mathrm{k}}$ in the $i$ bin has a reconstructed energy $E_{\text {deposited }}$ in the $j$ bin. . . . . . . . . . . . . 98

6.26 Event display of an electron shower candidate, showing the $1 \times 4 \mathrm{~cm}^{2}$ area used for the $d E / d x$ calculation. Each small black rectangle

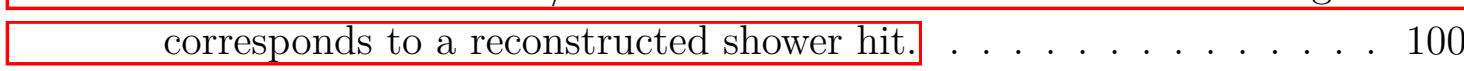

6.27 Area-normalised distributions of the $d E / d x$ median for simulated electrons (red) and photons (blue) produced in neutrino interactions. 101

6.28 Electromagnetic showers aligned with the wire orientation will have \begin{tabular}{|c|}
\hline a large pitch value and their measured $d E / d x$ will be shifted towards \\
\hline
\end{tabular} low values. . . . . . . . . . . . . . . . . . . . . . . . . . 101

6.29 Cross section of gammas on argon between $1 \mathrm{MeV}$ and $1 \mathrm{GeV}$. Here, \begin{tabular}{|c|}
\hline$\kappa$ refers to the pair production cross section for the nuclear field and \\
\hline electron field. Compton scattering is dominant below $10 \mathrm{MeV}$. From \\
\hline \hline 67
\end{tabular}

6.30 Pair production relative cross section as a function of the fraction of the photon energy $k$ transferred to either the electron or positron

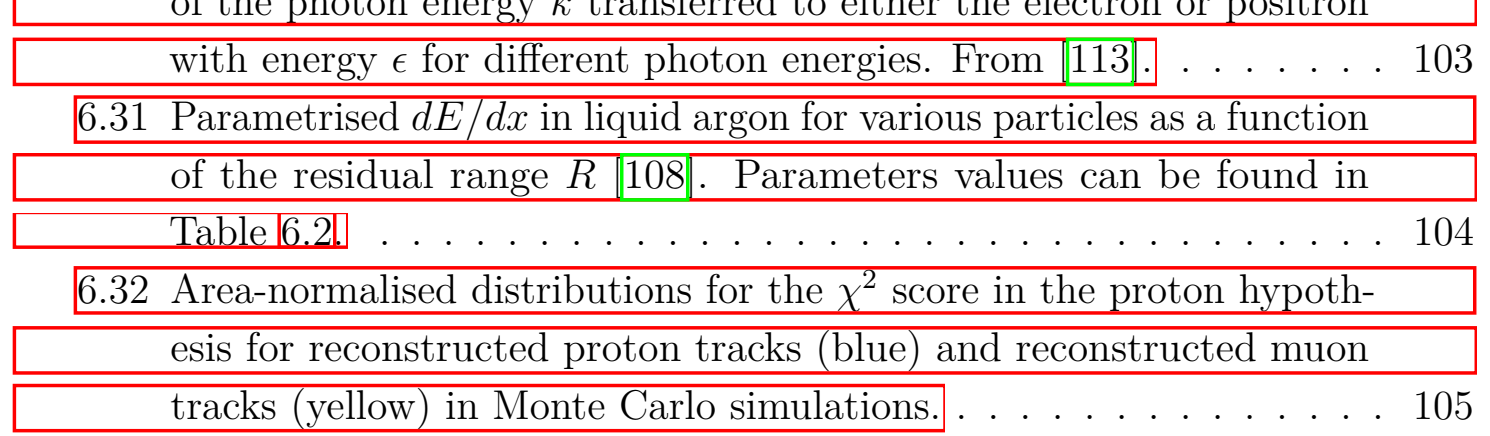


6.33 Reconstructed energy spectrum after the event selection algorithm

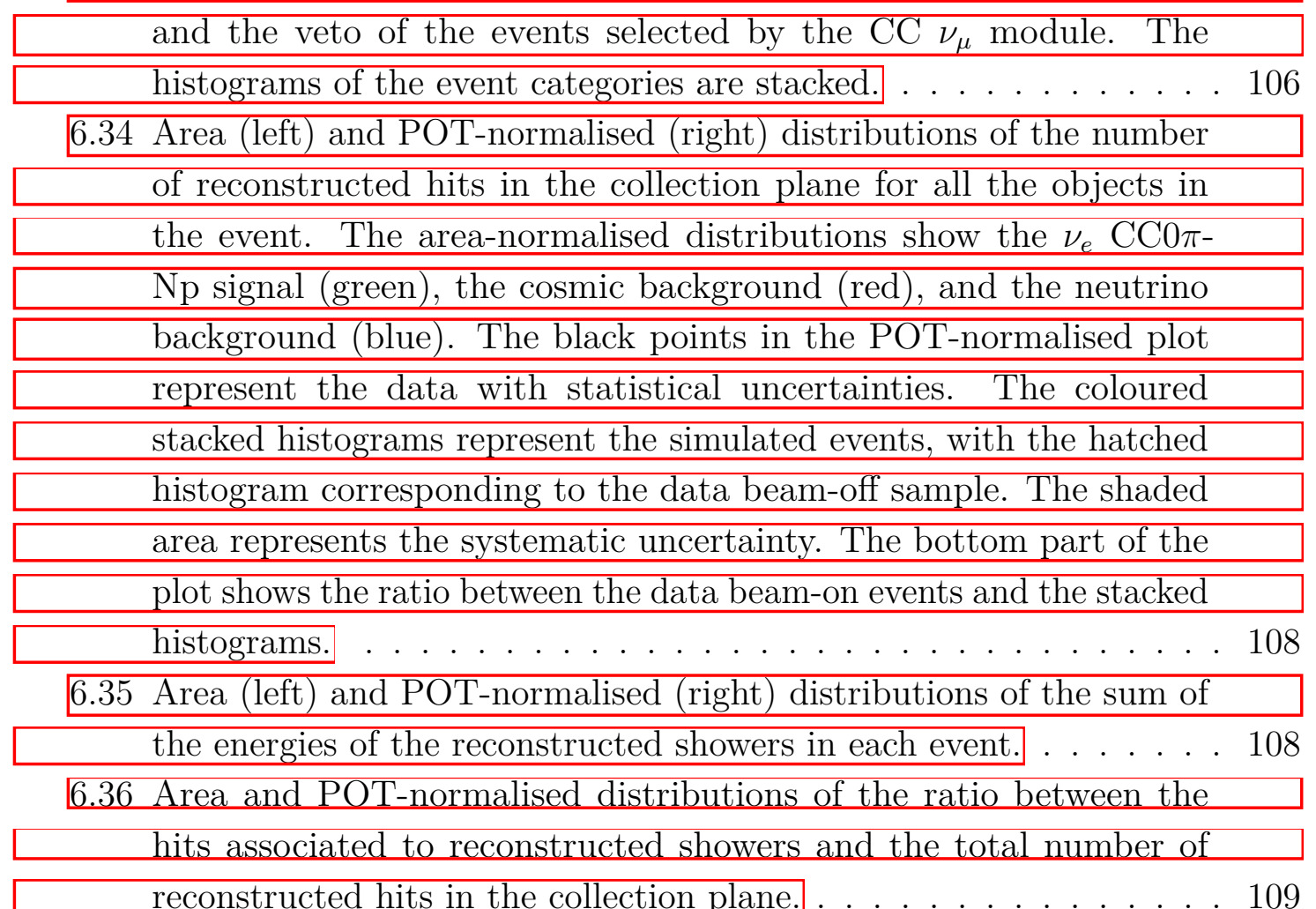

6.37 Area and POT-normalised distributions for reconstructed showers

$d E / d x$, classified according to the primary particle generating the

\begin{tabular}{|c|}
\hline \hline shower (left) and to the event category (right). In the generating- \\
\hline particle histogram, the data points correspond to the statistical \\
\hline
\end{tabular}

subtraction of the data beam-off events from the data beam-on events. 110

6.38 Area (bottom) and POT-normalised distributions of the distance between the reconstructed tracks and the reconstructed neutrino \begin{tabular}{|c|}
\hline vertex, classified according to the primary particle generating the \\
\hline
\end{tabular} shower (top left) and to the event category (top right). . . . . . . . 111

6.39 Area (bottom) and POT-normalised distributions of the distance between the reconstructed showers and the reconstructed neutrino vertex, classified according to the primary particle generating the

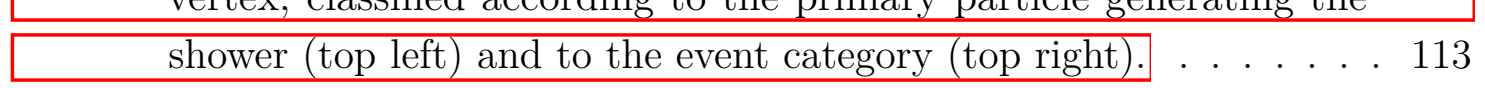

6.40 Area (bottom) and POT-normalised distributions of the proton $\chi_{p}^{2}$ score of the reconstructed tracks, classified according to the primary \begin{tabular}{|c|c|c|}
\hline particle generating the shower (top left) and to the event category \\
\hline
\end{tabular}

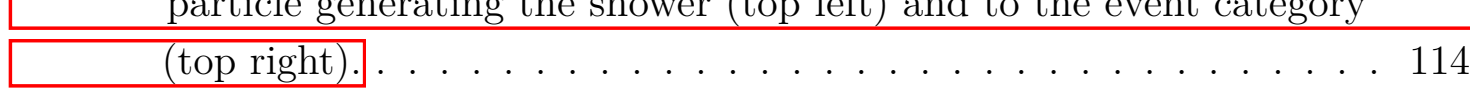

6.41 Area (bottom) and POT-normalised distributions of the angle $\alpha$ \begin{tabular}{|c|}
\hline between each reconstructed track and the leading shower, classified \\
\hline according to the primary particle generating the shower (top left) \\
\hline
\end{tabular}

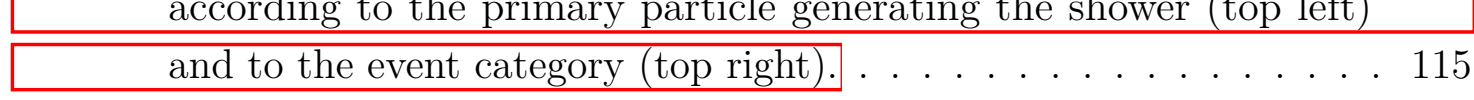


6.42 Area (bottom) and POT-normalised distributions of the reconstructed \begin{tabular}{|l|}
\hline tracks, classified according to the primary particle generating the \\
\hline shower (top left) and to the event category (top right). . . . . . . . 116 \\
\hline 6.43 Purity of the selected sample before (green) and after (orange) the \\
\hline application of the rectangular cuts as a function of the reconstructed \\
\hline energy $E_{\text {deposited }}$ Error bars are statistical only. . . . . . . . . . . . 117 \\
\hline
\end{tabular}

6.44 Selection efficiency (red) and purity (blue) of the selected sample

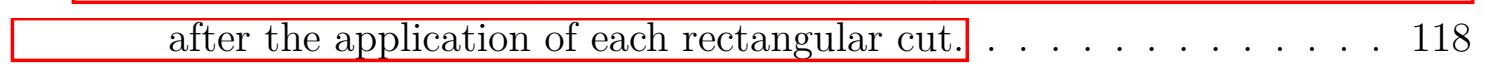

6.45 Reconstructed energy spectrum $E_{\text {deposited }}$ after the application of \begin{tabular}{|c|}
\hline the rectangular cuts. The black points represent the data with \\
\hline
\end{tabular} \begin{tabular}{l}
\hline statistical uncertainties. The coloured stacked histograms represent \\
\hline the simulated events, with the hatched histogram corresponding to \\
\hline the data beam-off sample. The shaded area represents the systematic \\
\hline uncertainty. . . . . . . . . . . . . . . . . . . . . . . . . . 118
\end{tabular}

6.46 Angular distribution of the selected Monte Carlo and data events after the application of the rectangular cuts. The black points represent the data with statistical uncertainties. The coloured stacked histograms represent the simulated events, with the hatched \begin{tabular}{|c|}
\hline histogram corresponding to the data beam-off sample. The shaded \\
\hline
\end{tabular} area represents the systematic uncertainty. . . . . . . . . . . . . 119

6.47 Event displays of the collection plane of background events not

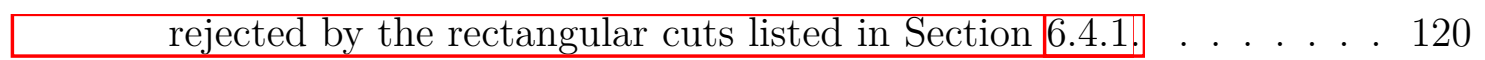

6.48 Coefficient function (6.22) of the AdaBoost boosting technique for

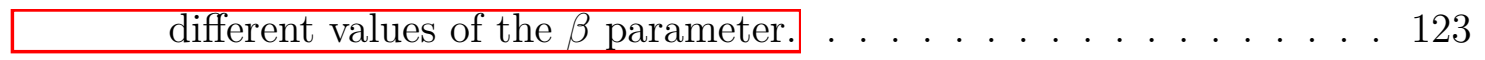

6.49 ROC curves for the three BDTs used in this analysis, showing the \begin{tabular}{|l|}
\hline background-rejection power as a function of the signal efficiency in \\
\hline a sample with 1000 signal events and 1000 background events. The \\
\hline points correspond to the signal efficiency and background-rejection \\
\hline
\end{tabular}

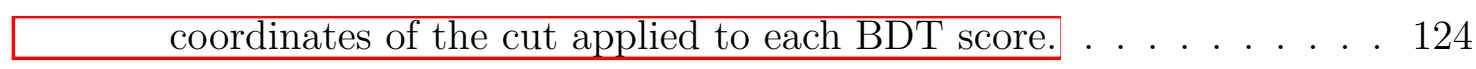

6.50 POT-normalised comparison between the data and Monte Carlo for \begin{tabular}{|c|}
\hline distributions of the BDTs score. The black points represent the \\
\hline
\end{tabular} \begin{tabular}{|l|}
\hline data with statistical uncertainties. The coloured stacked histograms \\
\hline represent the simulated events, with the hatched histogram corre- \\
\hline sponding to the data beam-off sample. The shaded area represents \\
\hline the systematic uncertainty. Vertical axis is in log-scale. . . . . . . . 125
\end{tabular}

6.51 Selection efficiency as a function of true neutrino energy after the \begin{tabular}{|c|}
\hline application of the rectangular cuts (orange), and after the application \\
\hline
\end{tabular} \begin{tabular}{|c|c|c|}
\hline of the BDTs (purple). Error bars are statistical only. . . . . . . 126 \\
\hline
\end{tabular} 
6.52 Purity of the sample after the event selection (green), after the \begin{tabular}{|c|}
\hline application of the rectangular cuts (orange), and after the application \\
\hline \hline of the BDTs (purple) as a function of the reconstructed energy \\
\hline$E_{\text {deposited. Error bars are statistical only. . . . . . . . . . . . . } 126}$ \\
\hline
\end{tabular}

6.53 Reconstructed energy spectrum $E_{\text {deposited }}$ after the application of

\begin{tabular}{|c|}
\hline the BDTs cuts. The black points represent the data with statisti- \\
\hline
\end{tabular}

\begin{tabular}{|l|}
\hline cal uncertainties. The coloured stacked histograms represent the \\
\hline simulated events, with the hatched histogram corresponding to the \\
\hline data beam-off sample. The shaded area represents the systematic \\
\hline
\end{tabular}

uncertainty. . . . . . . . . . . . . . . . . . 127

6.54 Angular distribution of the selected Monte Carlo and data events

\begin{tabular}{|c|}
\hline after the application of the BDTs cuts. The black points represent the \\
\hline
\end{tabular}

\begin{tabular}{|c|}
\hline data with statistical uncertainties. The coloured stacked histograms \\
\hline
\end{tabular}

\begin{tabular}{|c|}
\hline represent the simulated events, with the hatched histogram corre- \\
\hline
\end{tabular}

\begin{tabular}{|c|}
\hline sponding to the data beam-off sample. The shaded area represents \\
\hline
\end{tabular}

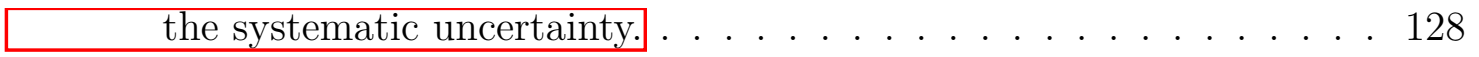

6.55 Selected events classified according to the type of neutrino interaction

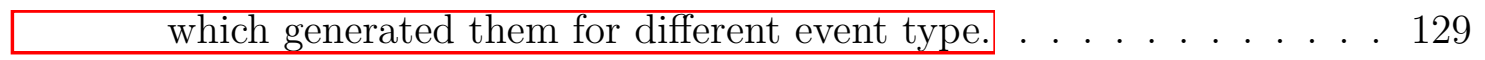

6.56 Distribution of the reconstructed showers $d E / d x$ after rectangular

\begin{tabular}{|c|}
\hline cuts (left) and BDTs cuts (right). The black points represent the \\
\hline
\end{tabular}

\begin{aligned} \hline statistical subtraction of the data beam-off events from the data \\ \hline\end{aligned}

\begin{tabular}{|c|}
\hline beam-on events. The coloured stacked histograms represent the \\
\hline
\end{tabular}

simulated events, classified according to the particle which generated

- the shower. . . . . . . . . . . . . . . . . . . 130

6.57 Reconstructed energy spectrum of the events selected with the NC-

\begin{tabular}{|c|c|}
\hline & it the data \\
\hline & statistical uncertainties. The coloured stacked histograms represent \\
\hline & the simulated events, with the hatched histogram corresponding to \\
\hline & the data beam-off sample. The shaded area represents the systematic \\
\hline & uncertainty. \\
\hline 6.58 & Reconstructed energy spectrum of the events selected with the CC $\nu_{\mu^{-}}$ \\
\hline & enhanced reverse cuts. The black points represent the data with \\
\hline & statistical uncertainties. The coloured stacked histograms represent \\
\hline & the simulated events, with the hatched histogram corresponding to \\
\hline & the data beam-off sample. The shaded area represents the systematic \\
\hline & uncertainty. The bottom part of the plot shows the ratio between \\
\hline & the data beam-on events and the stacked histograms. . . . . . . . . \\
\hline 6.59 & NuMI and BNB neutrino fluxes for each neutrino and antine \\
\hline & component, when the beams are in neutrino mode. \\
\hline
\end{tabular}


6.60 Number of selected events after the selection and rectangular cuts \begin{tabular}{|l|}
\hline described in Section 6.4 .1 , plus an additional threshold on the leading \\
\hline shower energy of $100 \mathrm{MeV}$. The simulated events are classified \\
\hline according to the event category. The number of events corresponds \\
\hline to $23 \times 10^{20} \mathrm{NuMI}$ POT in neutrino mode
\end{tabular} to $2.3 \times 10^{20}$ NuMI POT in neutrino mode. . . . . . . . . . . . . . 134

7.1 Pion production at a fixed angle as measured by the HARP experi-

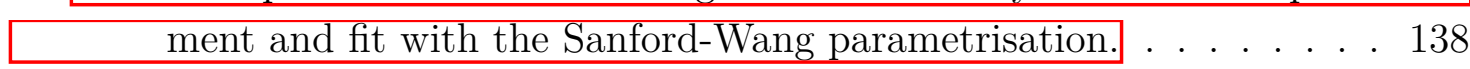

7.2 Selection efficiency, reconstructed energy spectrum, fractional covariance matrix, and correlation matrix obtained by varying the BNB

\begin{tabular}{|c|}
\hline flux parameters in 100 simulated universes. The colour scale for \\
\hline the selection efficiency and the energy spectrum corresponds to the \\
\hline number of universes. The red bars correspond to the central value \\
\hline and its flux systematic uncertainty only. The data beam-off sample \\
\hline
\end{tabular}

is not included in these plots. . . . . . . . . . . . . . . . . . 139

7.3 Selection efficiency, reconstructed energy spectrum, fractional covariance matrix, and correlation matrix obtained by varying the standard GENIE parameters in 100 simulated universes. The colour scale for \begin{aligned} \hline the selection efficiency and the energy spectrum corresponds to the & \end{aligned} \begin{tabular}{|l|}
\hline number of universes. The red bars correspond to the central value \\
\hline and its cross-section systematic uncertainty only. Events with MEC \\
\hline interactions are not included in these plots and the uncertainties do \\
\hline not include RPA effects. The data beam-off sample is not included \\
\hline
\end{tabular} in these plots. . . . . . . . . . . . . . . . . . . . . . . . 141

7.4 Reconstructed energy spectrum, fractional covariance matrix, and \begin{tabular}{|c|}
\hline correlation matrix obtained with the detector variations samples. The \\
\hline red bars correspond to the central value and its detector systematic \\
\hline uncertainty only. The data beam-off sample is not included in these \\
\hline
\end{tabular} uncertainty only. The data beam-off sample is not included in these plots. . . . . . . . . . . . . . . . . . . . . . . 144

$7.5 \quad$ Full fractional covariance (left) and correlation (right) matrices \begin{tabular}{|c|}
\hline obtained by combining the statistical, cross-section, flux, and detector \\
\hline uncertainties for the $E_{\text {deposited }}$ distribution before the background \\
\hline rejection. . . . . . . . . . . . . . . . . . . . . . . . . .
\end{tabular}

8.1 The MiniBooNE low-energy excess compared to the simulated MiniBooNE beam-intrinsic $\nu_{e}$ component. The error bars correspond to the data statistical uncertainties on observed data, and the shaded \begin{tabular}{|c|}
\hline region corresponds to the Monte Carlo systematic uncertainties. From \\
\hline
\end{tabular}

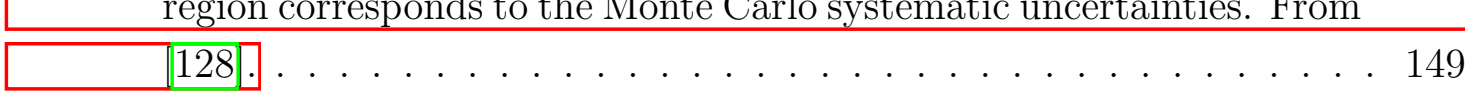

8.2 The MiniBooNE response matrix for the electron hypothesis of the

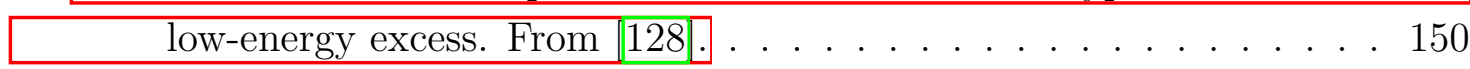


8.3 Unfolded MiniBooNE beam intrinsic $\nu_{e}$ Monte Carlo and data spectra in the electron hypothesis. It represents the result of the unfolding of Figure 8.1. The filled area correspond to the data unfolding uncertainty. From 128. . . . . . . . . . . . . . . . . . . . 151

8.4 Energy spectrum $E_{\text {deposited }}$ of the selected events stacked with the MiniBooNE low-energy excess signal in the electron hypothesis (light green). The black points represent the data with statistical uncertainties. The coloured stacked histograms represent the simulated events, with the hatched histogram corresponding to the data beamoff sample. The shaded area represents the systematic uncertainty. These distributions are identical to the ones in Figure [6.45|and Figure 6.53 , except for the low-energy excess signal component. . . . . . . 152

8.5 Expected sensitivity to the low-energy excess in the electron hypothesis as a function of a constant scaling of our selection efficiency and background-rejection power, with (up) and without (bottom) systematic uncertainties. The red line corresponds to the $5 \sigma$ contour. 156

9.1 Aerial view of the Fermilab campus with the position of the three detectors forming the future Short Baseline Neutrino program. The neutrino beam target is placed on the right side of the picture. From

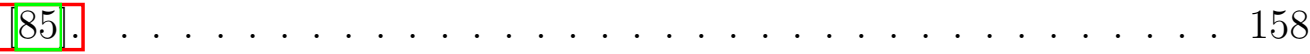

9.2 Sensitivity of the SBN Program to $\nu_{\mu} \rightarrow \nu_{e}$ oscillation signals. The filled areas correspond to the current allowed regions and the lines represent the sensitivity of the SBN program at different confidence levels. From $85 . \ldots \ldots \ldots$. . . . . . . . . . . . . . 159 


\section{List of Tables}

4.1 Systematic uncertainties on the BNB flux calculation. The other category includes uncertainties in pion and nucleon cross-sections on beryllium and aluminium, as well as the horn current calibration uncertainty, and uncertainty in the horn current distribution. . . . . 49

6.1 Summary of the selection results, showing the contribution of each event category, for a MicroBooNE exposure of $4.34 \times 10^{19}$ POT. Efficiency uncertainties are statistical only. For the Cosmic contaminated category the number of generated events correspond to the number of neutrino interactions inside the cryostat. For the Cosmic category, it corresponds to the total number of simulated neutrino interactions, both inside and outside the cryostat. . . . . . . . . . . . 86

6.2 Stopping power parametrisation for various particle types in liquid argon (from 108]). . . . . . . . . . . . . . . . . . . . . . 104

6.3 Summary of selected events in simulation and data with rectangular and BDTs cuts for a MicroBooNE exposure of $4.34 \times 10^{19}$ POT. The uncertainties on the simulated events are caused by systematic effects described in Section 7 . Uncertainties on the data events are statistical only. Numbers are rounded up to the first decimal digit. . 127

7.1 Summary of the detector uncertainties variations in the BNB+cosmic sample, broken down by event category. . . . . . . . . . . . . . . 145

7.2 Summary of the systematic uncertainties variations in the BNB+cosmic sample, break down by event category. . . . . . . . . . . . . . . . 146

8.1 Summary of the sensitivities to the MiniBooNE low-energy excess in the electron hypothesis for an exposure of the MicroBooNE detector of $13.2 \times 10^{20}$ POT. The uncertainties in the number of events include the systematic effects described in Section 77 . . . . . . . . . . . . . 153 


\title{
List of Abbreviations
}

\author{
ADC ... . Analog-to-Digital Converter \\ ASIC . . . A Application Specific Integrated Circuit \\ AdaBoost . . . Adaptive Boost \\ ArgoNeuT . . Argon Neutrino Test \\ BDT . . . . . Boosted Decision Tree \\ BNB ... . . Booster Neutrino Beam at Fermilab \\ BSM . . . . B Beyond the Standard Model of particle physics \\ CCDIS . . . C Charged-Current Deep Inelastic Scattering \\ CCQE ... . . Charged-Current Quasi-Elastic interaction \\ CCRES . . . C Charged-Current Resonant interaction \\ CC ...... Charged Current interaction \\ CNGS . . . . CERN Neutrinos to Gran Sasso \\ CP ..... Charge Parity \\ CRT . . . . . Cosmic-Ray Tagger at MicroBooNE \\ CUORE . . . . Cryogenic Undregroung Observatory for Rare Events \\ DAQ . . . . . Data Acquisition system \\ DAR .... . Decay-at-rest \\ DIF . . . . Decay-in-flight \\ DUNE . . . . Deep Underground Neutrino Experiment \\ EXO .... . Enriched Xenon Observatory \\ EXT . . . . External trigger at MicroBooNE \\ FEM . . . . . Front-End Module \\ FNAL . . . . Fermi National Accelerator Laboratory \\ FSI . . . . . Final State Interactions \\ FWHM ... . Full Width Half Maximum
}


GALLEX . . GALLium EXperiment at the Gran Sasso Laboratories

GEANT . . . . GEometry ANd Tracking simulation toolkit

GERDA . . . GERmanium Detector Array

GUT ... . . Grand Unification Theory

HARP . . . . Hadron Production experiment at CERN

HV ... . . . High Voltage

ICARUS . . . Imaging Cosmic And Rare Underground Signal

KARMEN . . KArlsruhe Rutherford Medium Energy Neutrino experiment

KATRIN . . . KArlsruhe TRItium Neutrino experiment

LArTPC . . . Liquid Argon Time Projection Chamber

LEP . . . . . Large Electron-Positron Collider at CERN

LINAC . . . Linear Accelerator at Fermilab

LSND . . . . L Liquid Scintillator Neutrino Detector, neutrino detection experiment at Los Alamos

MCS . . . . . Multiple Coulomb Scattering

MEC . . . . . Meson Exchange Current

MIP . . . . Minimum-Ionising Particle

MPV ... . Most Probable Value

MSW .... Mikheyev-Smirnov-Wolfenstein effect, also called matter effect

MicroBooNE . Micro Booster Neutrino Experiment

MiniBooNE . Mini Booster Neutrino Experiment

MuCS . . . Muon Counter System at MicroBooNE

NC . . . . . Neutral Current interaction

NEXT . . . . Neutrino Experiment with a Xenon TPC

NuMI . . . . Neutrino at Main Injector beam at Fermilab

OPERA . . . O Oscillation Project with Emulsion-Tracking Apparatus experiment

PE ...... Photoelectron

PMNS . . . . Pontecorvo-Maki-Nakagawa-Sakata mixing matrix of the neutrino sector

PMT . . . . Photomultiplier Tube 
POT .... Protons-on-target

RFG . . . . Relativistic Fermi Gas

RFQ .... . Radio Frequency Quadrupole

RMS .... . Root Mean Square

ROC .... Receiver Operating Characteristic

ROI ... . Region of interest

RPA ... . . Random Phase Approximation

RTD . . . . Resistive Thermal Device

SAGE . . . . Soviet American Gallium Experiment

SBN . . . . Short Baseline Neutrino program at Fermilab

SDK . . . . Software Development Kit

SNEWS . . . SuperNova Early Warning System

SNO . . . . Solar Neutrino Observatory

SVM ... . Support Vector Machine

SciBooNE . . SciBar Booster Neutrino Experiment

V-A . . . . Vector - Axial current in the electroweak interaction 
Secondo la proposta di Pauli si può, ad esempio, ammettere l'esistenza di una nuova particella, il così detto neutrino avente carica elettrica nulla e massa dell'ordine di grandezza di quella dell'elettrone o minore.

- Enrico Fermi 1

\section{1 \\ Introduction}

This thesis describes the first fully-automated electron neutrino search in a Liquid Argon Time Projection Chamber (LArTPC) and the work towards the search for a low-energy excess of electron neutrinos.

The history of neutrinos begins with an anomaly: the continuous energy spectrum of the nuclear beta decay could not be explained with the presence of only two particles in the final state. It took more than 25 years to experimentally confirm their existence, but neutrinos continued to puzzle experimentalists and theorists until the early 2000s, when the existence of neutrino oscillation was finally settled. Furthermore, in the last two decades, several experiments collected results not fully in agreement with a 3-neutrino scenario. In particular, the Liquid Scintillator Neutrino Detector (LSND) first, and MiniBooNE later, found an excess of electron-like events, which could be explained with the existence of a fourth, non-weakly-interacting, neutrino. However, the MiniBooNE experiment is a Cherenkov detector and is not able to distinguish between single photons and electrons in the final state. The LArTPC technology, instead, offers excellent granularity and powerful separation between electrons and photons. The goal of the MicroBooNE experiment is to search for, and definitely clarify the observation of this electron-like low-energy excess.

In Chapter 2 we will provide a brief theoretical introduction to neutrino oscillations and to the main experimental techniques employed to observe them. 
In Chapter 3, the LSND and MiniBooNE experiments will be described. A brief overview of other anomalous results and their possible theoretical interpretations will also be provided. The MicroBooNE experiment will be described in Chapter 4 We will enumerate the main physics goals of the experiment and explain the main features of the detector, with an overview of the Booster Neutrino Beam at Fermilab. The techniques employed to reconstruct the signals coming from the detector will be described in Chapter 5. The pattern recognition is performed by the Pandora framework, which will be briefly outlined. Chapter 6 will thoroughly characterise the fully automated electron-neutrino selection, which aims to obtain a sample enriched with $\nu_{e} \mathrm{CC} 0 \pi-\mathrm{Np}$ interactions. Two background-rejection techniques will be used: one with rectangular cuts on kinematic and calorimetric variables and one employing Boosted Decision Trees. The energy reconstruction and the measurement of the energy loss per length, essential for electron/photon separation, will be outlined. The validation of the selection will be performed with a study on orthogonal side-bands enriched with neutral-current and charged-current $\nu_{\mu}$ interactions and by applying the selection on an independent data sample, containing neutrino interactions from the NuMI beam. The systematic uncertainties in the selection, caused by cross section, flux, and detector effects, will be estimated in Chapter 7 In Chapter 8 the sensitivity to the low-energy excess of the MiniBooNE experiment in the electron hypothesis will be calculated. The performances required to reach a $5 \sigma$ sensitivity will also be evaluated. The thesis ends in Chapter 9 with a summary of the results and an overview of the future prospects for MicroBooNE and for neutrino physics in general.

Appendix A contains a publication, whose corresponding author is the author of this thesis, with the first measurement of the cosmic-ray reconstruction efficiency in a LArTPC. 


\section{2}

Neutrino physics

\section{Contents}

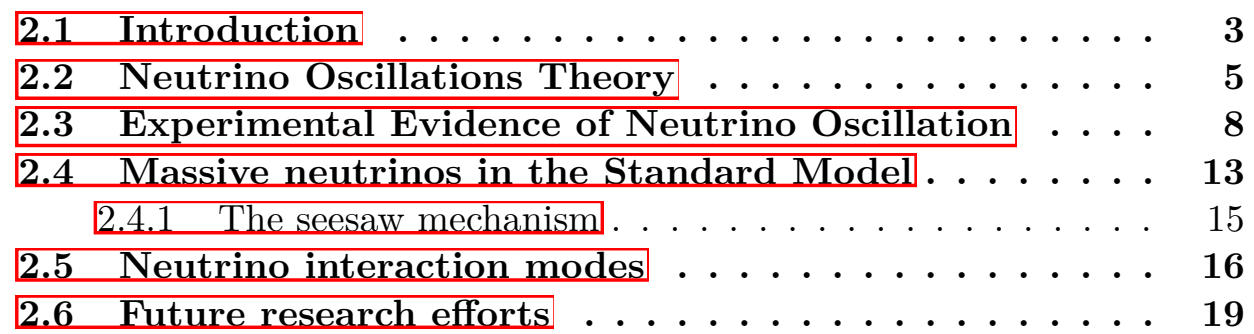

This chapter includes a brief historical overview and a review of the introductory theoretical background of neutrino physics. In particular, the mechanism of neutrino oscillations will be described and several experimental techniques employed to detect them will be reviewed. The existence of neutrino oscillations implies non-null neutrino masses and a mechanism to explain them will be outlined. The modes of neutrino interaction in the matter will also be presented.

\section{$2.1 \quad$ Introduction}

Neutrino physics represents one of the most exciting areas of active research in particle physics. The history of the early days of particle physics shows that neutrinos have challenged physicists since the famous Pauli's letter to his fellow 


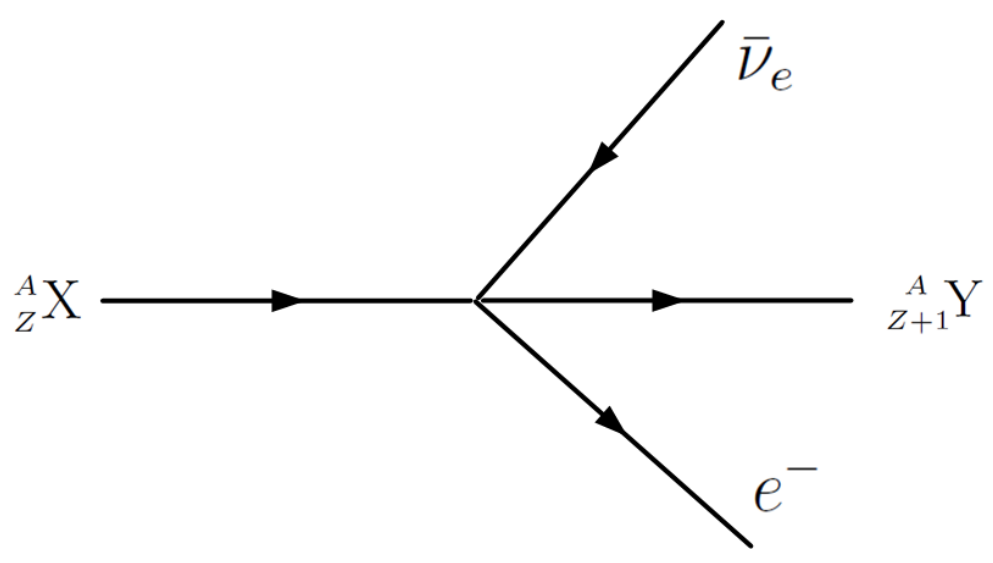

Figure 2.1: Feynman diagram of the $\beta$-decay of a ${ }_{Z}^{A} X$ into a ${ }_{Z+1}^{A} Y$ nucleus in the Fermi approximation.

Radioactive Ladies and Gentlemen [2], where he postulated the existence of a new neutral particle to explain the continuous spectrum of the nuclear $\beta$-decay. Fermi hypothesised the neutrino to be emitted in the three-body process:

$$
n \rightarrow p+e^{-}+\bar{\nu}_{e}
$$

and mediated by a four-fermion interaction in the form of:

$$
\frac{G_{F}}{\sqrt{2}}\left(\bar{n} \Gamma_{N} p\right)\left(\bar{\nu}_{e} \Gamma_{L} e\right)
$$

where, in modern terms, $G_{F}$ is the Fermi constant and $\Gamma_{N, L}$ are a linear combination of the gamma matrices. The Feynman diagram of the four-fermion approximation for the $\beta$-decay is shown in Figure 2.1.

This theory paved the way for the first experimental direct detection of neutrinos by Cowan and Reines in 1956 [3], which exploited the inverse $\beta$-decay process:

$$
\bar{\nu}_{e}+p \rightarrow e^{+}+n
$$

The key detection technique, still used in modern reactor experiments, employed the detection of the $e^{+} e^{-} \rightarrow 2 \gamma$ annihilation in delayed coincidence with the $\gamma$ emitted by the capture of the recoiling neutron shortly afterwards.

The leptonic current $\bar{\nu}_{e} \Gamma_{L} e$ was later hypothesised to be left-handed in the form of $\gamma_{\mu}\left(1-\gamma_{5}\right)$ by Feynman and Gell-Mann $(V-A$ theory) 4 . For massless 
neutrinos, this allows assigning a left-handed (right-handed) helicity to neutrinos (anti-neutrinos), which was experimentally verified by Goldhaber 5.

\subsection{Neutrino Oscillations Theory}

In the modern Standard Model of particle physics there are three flavours of (anti)neutrinos $\left(\stackrel{\leftrightarrow}{\nu}_{e}, \stackrel{\leftrightarrow}{\nu}_{\mu}, \stackrel{\leftrightarrow}{\nu}_{\tau}\right)$, each one paired to a charged (anti)lepton $\left(e^{(+)}, \mu^{(+)}\right.$, $\tau^{(+)}$respectively). However, if neutrinos have masses, it is possible to have three (or more) neutrino mass eigenstates $\left(\nu_{1}, \nu_{2}, \nu_{3}, \ldots\right)$ analogues of the charged-lepton mass eigenstates. In this case, a neutrino produced as a flavour eigenstate would oscillate through its path and change to another flavour eigenstate. This happens because the flavour eigenstate is a mixture of the three (or more) mass eigenstates, which travel with different wavelengths and create interference patterns.

The oscillation probabilities can be derived in the case of two neutrino generations, which we report here for didactic reasons largely following the approach in 6. The flavour eigenstates $\nu_{\alpha}, \nu_{\beta}$ can be expressed as a superposition of the two mass eigenstates $\nu_{1}$ and $\nu_{2}$ using the nominal rotation matrix $U$ :

$$
U=\left[\begin{array}{cc}
\cos \theta & -\sin \theta \\
\cos \theta & \sin \theta
\end{array}\right] .
$$

The flavour neutrino $\nu_{\alpha}$ will then propagate as:

$$
\left|\nu_{\alpha}\right\rangle=\cos \theta\left|\nu_{1}\right\rangle+\sin \theta\left|\nu_{2}\right\rangle
$$

The time evolution of this superposition can be written, in the plane-wave assumption, as:

$$
|\nu(\vec{x}, t)\rangle=\cos \theta e^{-i p_{1} x}\left|\nu_{1}\right\rangle+\sin \theta e^{-i p_{1} x}\left|\nu_{2}\right\rangle
$$

If the neutrino is ultra-relativistic the exponential argument becomes:

$$
\begin{aligned}
p_{i} x & =E_{i} t-\vec{p}_{i} \vec{x} \simeq\left(E_{i}-p_{z, i}\right) L \\
& =\left(E_{i}^{2}-|\vec{p}|^{2}\right) /\left(E_{i}-p_{z, i}\right) L \\
& \simeq m_{i}^{2} / 2 E_{i} L \simeq m_{i}^{2} / 2 E L,
\end{aligned}
$$




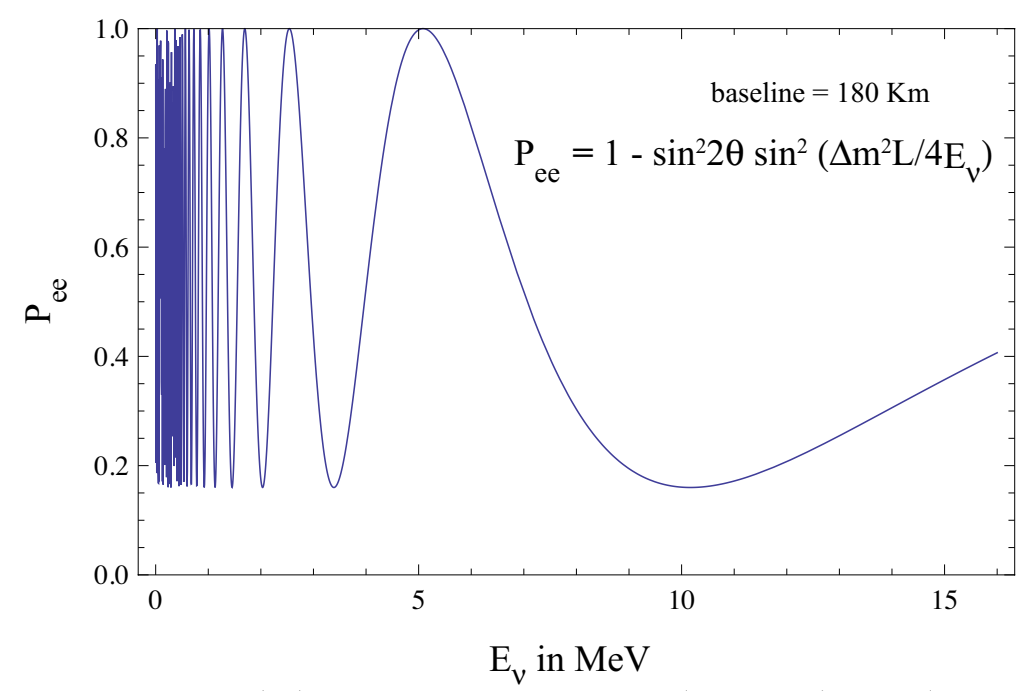

Figure 2.2: The $\nu_{e}$ survival probability $P\left(\nu_{e} \rightarrow \nu_{e}\right)$ as a function of the neutrino energy for $L=180 \mathrm{~km}, \Delta m^{2}=7.0 \times 10^{-5} \mathrm{eV}^{2}$ and $\sin ^{2} \theta=0.84$.

and the oscillation probability of the neutrino with flavour $\alpha$ can be written as:

$$
\begin{aligned}
P_{\alpha \alpha} & =\left|\left\langle\nu_{\alpha} \mid \nu(L)\right\rangle\right|^{2} \\
& =1-\sin ^{2} 2 \theta \sin ^{2}\left(\frac{\Delta m^{2} L}{4 E}\right),
\end{aligned}
$$

where $\Delta m^{2} \equiv m_{2}^{2}-m_{1}^{2}$ is the mass splitting between the two mass states at play. The probability of observing a neutrino of flavour $\beta$ will be then given by:

$$
P_{\alpha \beta}=1-P_{\alpha \alpha}=\sin ^{2} 2 \theta \sin ^{2}\left(\frac{\Delta m^{2} L}{4 E}\right) .
$$

Equations 2.8 and 2.9 show that the amplitude of the oscillation is regulated by the rotation angle $\theta$, while its frequency depends on the mass splitting, at a fixed $L / E$ ratio. Figure 2.2 shows as an example the $\nu_{e}$ survival probability as a function of the neutrino energy for $L=180 \mathrm{~km}, \Delta m^{2}=7.0 \times 10^{-} 5 \mathrm{ev}^{2}$ and $\sin ^{2} \theta=0.84$.

Neutrino oscillations experiments can be divided into two main categories: disappearance experiments, which measure the deficit of neutrinos of a certain flavour (measuring $P_{\alpha \alpha}$ ), and appearance experiments, which look for an excess of neutrinos of a certain flavour (measuring $P_{\alpha \beta}$ ).

The $U$ matrix can be easily extended in the case of the three generations of neutrinos $\nu_{e}, \nu_{\mu}$, and $\nu_{\tau}$. In this case, the flavour eigenstates mixing is obtained from:

$$
\left[\begin{array}{l}
\nu_{e} \\
\nu_{\mu} \\
\nu_{\tau}
\end{array}\right]=\left[\begin{array}{lll}
U_{e 1} & U_{e 2} & U_{e 3} \\
U_{\mu 1} & U_{\mu 2} & U_{\mu 3} \\
U_{\tau 1} & U_{\tau 2} & U_{\tau 3}
\end{array}\right]\left[\begin{array}{c}
\nu_{1} \\
\nu_{2} \\
\nu_{3}
\end{array}\right]
$$


where the rotation is given by the so-called Pontecorvo-Maki-Nakagawa-Sakata (PMNS) matrix. It is also possible to parametrise the $U$ matrix in the following useful way:

$$
U_{\mathrm{PMNS}}=\left[\begin{array}{ccc}
1 & 0 & 0 \\
0 & c_{23} & s_{23} \\
0 & -s_{23} & c_{23}
\end{array}\right]\left[\begin{array}{ccc}
c_{13} & 0 & s_{13} e^{-i \delta_{C P}} \\
0 & 1 & 0 \\
-s_{13} e^{i \delta_{C P}} & 0 & c_{13}
\end{array}\right]\left[\begin{array}{ccc}
c_{12} & s_{12} & 0 \\
-s_{12} & c_{12} & 0 \\
0 & 0 & 1
\end{array}\right]
$$

where $s_{i j}\left(c_{i j}\right)$ is an abbreviation for $\sin \theta_{i j}\left(\cos \theta_{i j}\right)$ and $\delta_{C P}$ is the CP-violating phase. The mixing angles $\theta_{12}, \theta_{13}$, and $\theta_{23}$ are defined by:

$$
\tan ^{2} \theta_{12} \equiv \frac{\left|U_{e 2}\right|^{2}}{\left|U_{e 1}\right|^{2}}, \quad \tan ^{2} \theta_{23} \equiv \frac{\left|U_{\mu 3}\right|^{2}}{\left|U_{\tau 3}\right|^{2}}, \quad \sin \theta_{13} \equiv U_{e 3} e^{i \delta_{C P}}
$$

With three neutrino flavours the squared mass-splitting terms are $\Delta m_{12}^{2}$ and $\Delta m_{13}^{2}$. Being squared mass differences, we do not know the absolute ordering of the three masses: it is possible to have $m_{3}>m_{2}>m_{1}$ (normal hierarchy) or $m_{3}>m_{1}>m_{2}$ (inverted hierarchy). Customarily, $\Delta m_{12}^{2}$ and $\Delta m_{13}^{2}$ are also called $\left(\Delta m^{2}\right)_{\text {sol }}$ and $\left(\Delta m^{2}\right)_{\text {atm }}$, respectively, since they are usually measured with "solar" and "atmospheric" neutrinos. The situation is illustrated in Figure 2.3.

The mass splittings also determine the $L / E$ ratio at which the oscillation probability is maximised. In the assumption of two-neutrino oscillation, the oscillation frequency in Equation 2.8 becomes:

$$
\frac{\Delta m^{2} L}{4 E}=1.267\left(\frac{L}{\mathrm{~km}}\right)\left(\frac{\Delta m^{2}}{\mathrm{eV}^{2}}\right)\left(\frac{\mathrm{GeV}}{E}\right)
$$

which gives for $\theta_{12}, \theta_{13}$, and $\theta_{23}$ a maximum oscillation probability at $\approx 10^{4} \mathrm{~km} / \mathrm{GeV}$, $\approx 10^{2} \mathrm{~km} / \mathrm{GeV}$, and $\approx 10^{2} \mathrm{~km} / \mathrm{GeV}$, respectively. The $\theta_{12}, \theta_{13}$, and $\theta_{23}$ mixing angles are also known as the solar, reactor, and atmospheric mixing angles, since they maximise the oscillation probability at the typical $L / E$ ratios for solar, reactor, and atmospheric neutrinos. In the parametrisation of the $U_{\mathrm{PMNS}}$ matrix of Equation 2.11 the product of the matrices can then be written as:

$$
U_{\mathrm{PMNS}}=U_{\mathrm{atm}} \cdot U_{\text {reactor }} \cdot U_{\mathrm{sol}} \cdot
$$




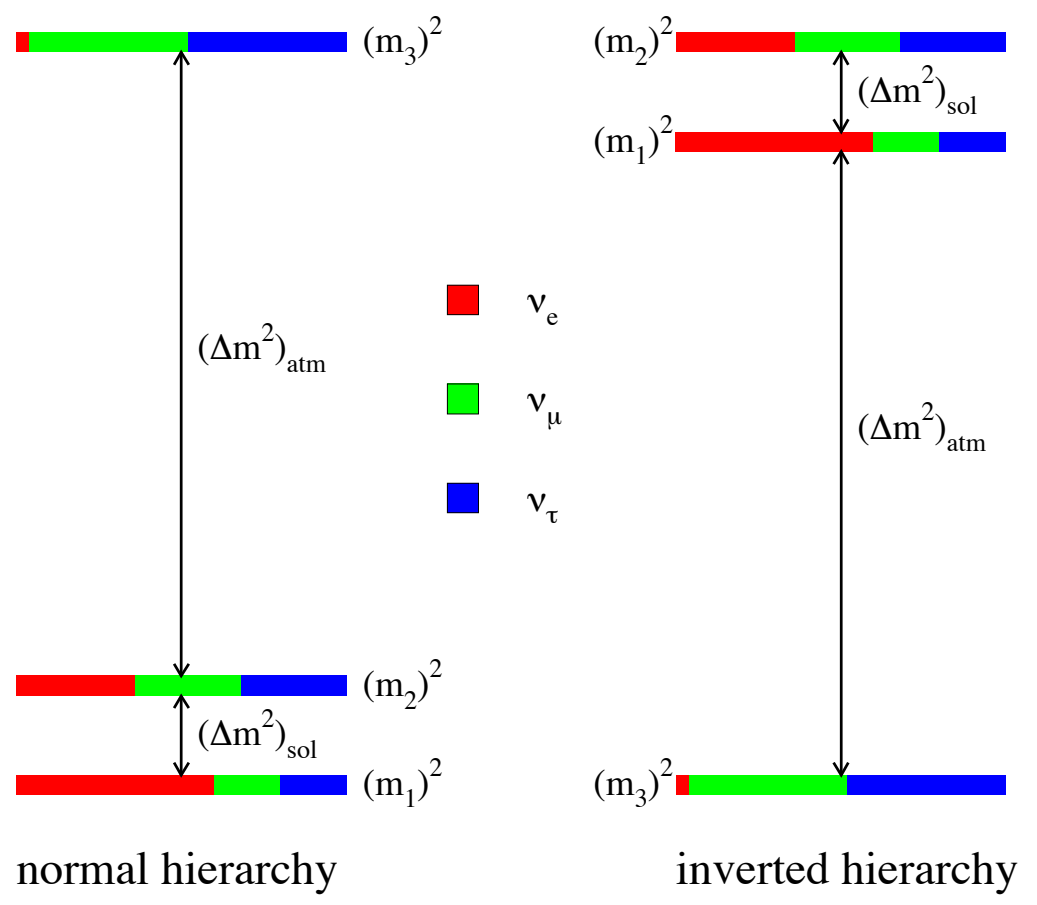

Figure 2.3: Diagram of the normal and inverted hierarchies. The colours correspond to the fraction of each distinct flavour contained in the mass eigenstate. From 7 .

\subsection{Experimental Evidence of Neutrino Oscilla- tion}

After the first direct detection of electron (anti)neutrinos by Cowan and Reines at the Savannah nuclear reactor $[3$, efforts were made in order to observe the other two neutrino flavours. In 1962, Lederman and others 8 first saw evidence of muon neutrinos interacting in the target and producing muons, while the DONUT collaboration finally observed the $\nu_{\tau}$ in 2001 [

The number of light neutrino species (meaning $m_{\nu}<m_{Z} / 2$ ) weakly interacting was also determined by precision measurements of the $Z$ boson width at LEP and SLD 10 as:

$$
N_{\nu}=\frac{\Gamma_{\text {inv }}}{\Gamma_{l}}\left(\frac{\Gamma_{l}}{\Gamma_{\nu}}\right)_{\mathrm{SM}}=2.984 \pm 0.008
$$

where $\Gamma_{l}$ is the leptonic decay width and $\Gamma_{\text {inv }}$ is the invisible decay width, assumed to be caused by $Z \rightarrow \nu \bar{\nu}$ decays. The lepton universality requires each neutrino 
flavour to contribute equally. This measurement does not forbid the existence of heavy $\left(m_{\nu}>m_{Z} / 2\right)$ or sterile (not weakly-interacting) neutrinos.

However, the number of neutrino interactions observed by several experiments was in disagreement with the one predicted by the theory of three massless neutrinos. In particular, two types of anomalies were identified, one involving the detection of solar neutrinos and one involving the neutrinos produced by cosmic rays in the atmosphere.

Solar neutrino anomaly The experiment by Ray Davis and others at Homestake was the first to directly detect the neutrinos produced in the sun 11 by the ${ }^{7} \mathrm{Be}$ and ${ }^{8} \mathrm{~B}$ decays (Figure 2.4).

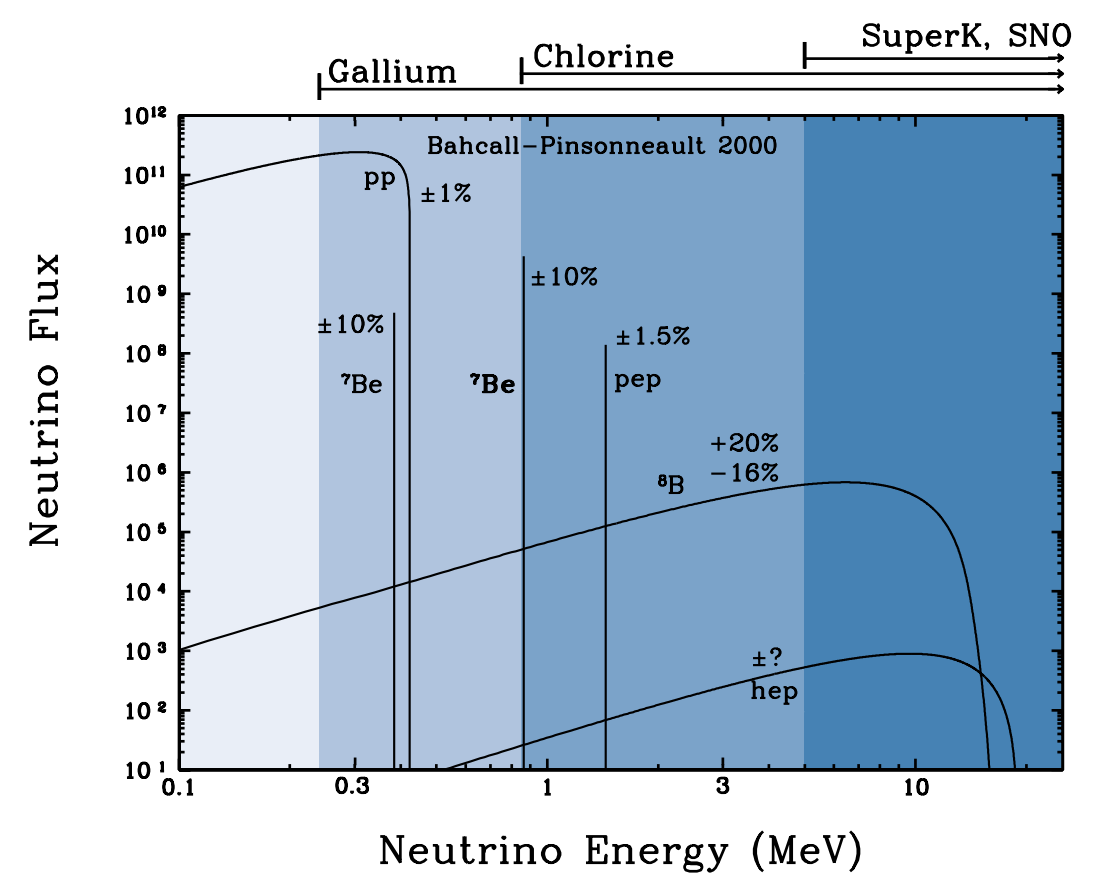

Figure 2.4: The solar neutrino energy spectrum predicted by the solar standard model. From $[12$.

Even if successfully detected, the solar neutrino interactions were less than expected and this deficit was later confirmed by several other experiments, including the Kamioka Observatory in Japan [13], the SAGE experiment in Russia [14, and the GALLEX experiment in Italy 15. The SNO (Sudbury Neutrino Observatory) experiment finally proved in 2001 that the cause of the deficit was the oscillation of 
the solar neutrinos into a different flavour eigenstate 16 , by measuring both the solar $\nu_{e}$ flux and the total solar neutrino flux, as shown in Figure 2.5.

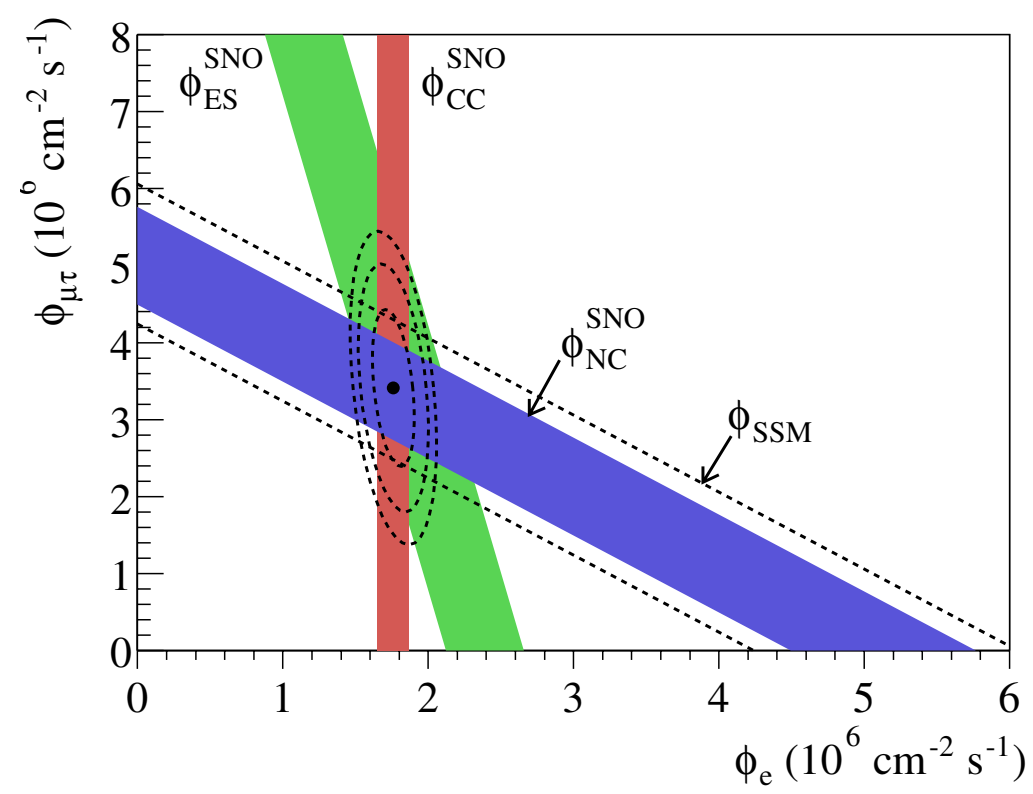

Figure 2.5: SNO measurement of the neutral-current $\left(\phi_{\mathrm{NC}}\right)$, charged-current $\left(\phi_{\mathrm{CC}}\right)$, and elastic scattering $\left(\phi_{\mathrm{ES}}\right)$ fluxes. The CC measurement is sensitive to the $\nu_{e}$ flavour only, while the $\mathrm{NC}$ and ES fluxes depend both on $\nu_{e}$ and $\nu_{\mu} / \nu_{\tau}$ interactions. The neutrino flux predicted by the SSM (Standard Solar Model) is given by $\phi_{\mathrm{SSM}}$.

The propagation of neutrinos in the matter is characterised by the MikheyevSmirnov-Wolfenstein (MSW) effect 17]: in presence of a high density of electrons, electron neutrinos experience a charged current coherent forward scattering. This cause the electron neutrinos to have a different effective mass when they propagate in a high-density medium, modifying the oscillation pattern.

The MSW effect is particularly important to explain the solar neutrino flux (Figure 2.4). Assuming a very high electron density in the sun core and an exponentially decreasing abundance (which are both good approximations in the standard solar model), the probability to observe an electron neutrino when it reaches the Earth is $P_{e e} \approx \sin ^{2} \theta$ for neutrinos above $2 \mathrm{MeV}$ (where the matter effect dominates) 6].

Atmospheric neutrino anomaly Cosmic muons decaying in the atmosphere produce a broad flux of muon (anti)neutrinos. The first underground water 
Cherenkov detectors, the IMB experiment and the Kamioka observatory, observed a discrepancy in the double ratio of muon to electron neutrinos, measured to expected. The evidence that this discrepancy was caused by the disappearance of atmospheric neutrinos was provided by the Super-Kamiokande experiment in 1998 18.

Figure 2.6 shows the zenith angle distributions of events detected by the SuperKamiokande experiment: the data points are in disagreement with the no-oscillation model and they can be interpreted as $\nu_{\mu} \leftrightarrow \nu_{\tau}$ oscillation.

In honour of these discoveries, the Nobel Prize in Physics 2015 was awarded to Takaaki Kajita (Super-Kamiokande) and Arthur B. McDonald (SNO).
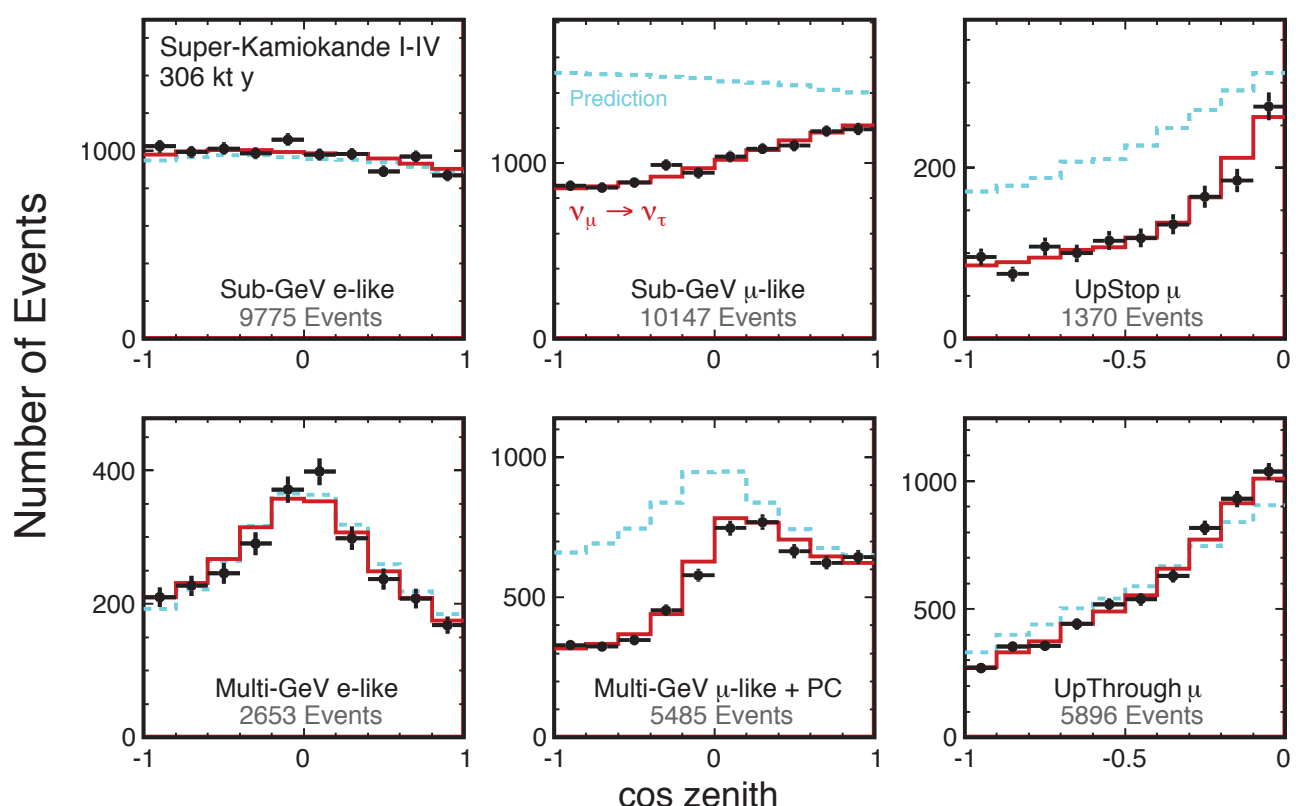

Figure 2.6: Zenith angle distributions of $\mu$-like and e-like events in the sub-GeV and multi-GeV data sets, as collected by the Super-Kamiokande experiment. The dashed blue line corresponds to the no-oscillation model. The red solid line represent the best fit to $\nu_{\mu} \leftrightarrow \nu_{\tau}$ oscillation (from $[19]$ ).

The KamLAND experiment finally spectacularly proved the oscillation pattern and the MSW model by measuring the reactor electron antineutrinos survival probability as a function of $L / E$, shown in Figure 2.7 .

Less than 20 years after the definitive confirmation of neutrino oscillations, the mixing angles and the mass splittings are all known with a relative uncertainty smaller than $5 \%$. The least-known parameter in the PMNS matrix is the $\delta_{C P}$ phase, 


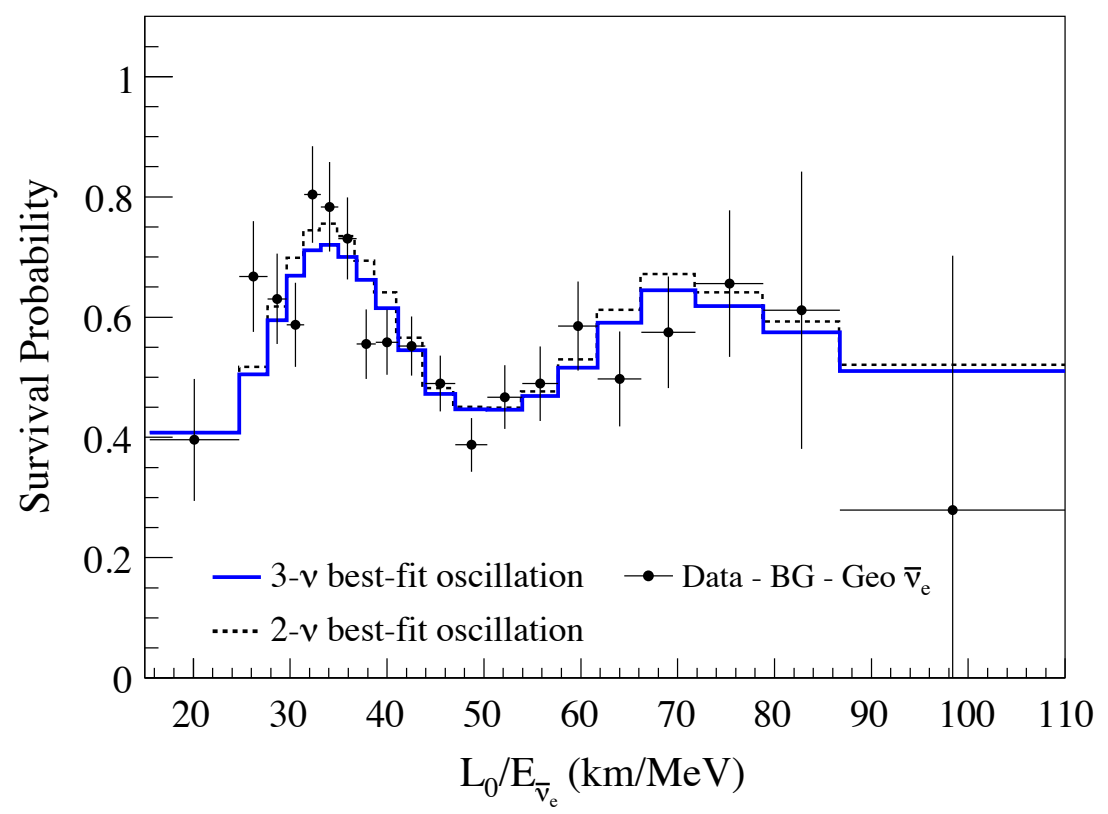

Figure 2.7: Ratio of the observed $\bar{\nu}_{e}$ spectrum to the expectation for no-oscillation versus $L_{0} / E$ for the KamLAND data. $L_{0}=180 \mathrm{~km}$ is the flux-weighted average reactor baseline (from $[20 \mid$ ).

which, if different from $0^{\circ}$ (or from $180^{\circ}$ ), would imply CP-violation in the leptonic sector. This parameter can be measured only with appearance experiments, where it is possible to verify if $P\left(\nu_{\alpha} \rightarrow \nu_{\beta}\right) \neq P\left(\bar{\nu}_{\alpha} \rightarrow \bar{\nu}_{\beta}\right)$. Recent results from the T2K and NOVA experiments give a best fit of $\delta_{C P}{ }^{\circ}=215_{-28}^{+40}\left(\delta_{C P} /^{\circ}=284_{-29}^{+27}\right)$ at $1 \sigma$ in the normal hierarchy (inverted hierarchy) model [21. A definitive measurement of the CP-violating phase would have far-reaching consequences in particle physics and cosmology, since it could explain the matter-antimatter asymmetry $[22$.

A precise estimation of the MSW effect is particularly important for a correct measurement of the CP violation. Since the matter contains electrons and not positrons, the electron neutrinos will experience a MSW effect opposite to the one experienced by antineutrinos. This difference will, in turn, modify the appearance probabilities, leading to $P\left(\nu_{e} \rightarrow \nu_{\mu}\right) \neq P\left(\bar{\nu}_{e} \rightarrow \bar{\nu}_{\mu}\right)$. This effect must the be disentangled from the CP-violating effect caused by the complex phases in the PMNS matrix.

The MSW effect is also dependent on the sign of the mass splitting $\Delta m_{31}$, which offers a way to resolve the hierarchy problem [23]. In this document, however, we will 
focus on short-baseline neutrino experiments, where the MSW effect is negligible.

\subsection{Massive neutrinos in the Standard Model}

The observation of neutrino oscillations poses a challenge for the SM since it requires a mechanism able to give mass to the neutrinos. In the standard formulation of the SM, quarks and leptons can be represented by a four-component Dirac spinor field $\psi_{D}$. This field can be decomposed into left-handed and right-handed twocomponent spinors with the chirality operators $\chi_{R}=\left(1+\gamma_{5}\right) \psi_{D}$ and $\chi_{L}=\left(1-\gamma_{5}\right) \psi_{D}$, respectively. The mass term for these spinors can be generated through the Higgs mechanism, which introduces a Dirac mass term in the Lagrangian:

$$
-\mathcal{L}_{D}=m_{D} \bar{\psi}_{D} \psi_{D}=m_{D}\left(\bar{\nu}_{L} \nu_{R}+\bar{\nu}_{R} \nu_{L}\right)
$$

This term breaks chirality symmetry, making helicity non-Lorentz-invariant, and can be applied also to neutrinos in an extension to the Standard Model. In this case, the neutrino would be a four-component massive Dirac spinor, just as any other fermion, with two components non-interacting. However, the current upper limit on the sum of the neutrino masses is $\sum m_{\nu}<0.12 \mathrm{eV}$ using cosmological constraints 24 and $\sum m_{\nu}<2 \mathrm{eV}$ from $\beta$-decay experiments 25. The KATRIN experiment will directly measure the neutrino mass through a precise reconstruction of the tritium $\beta$-decay spectrum, with an expected sensitivity of $0.2 \mathrm{eV} 26$. These results require a Yukawa coupling six orders of magnitude smaller than the electron one, which is not considered natural.

Most importantly, right-handed (left-handed) neutrinos (antineutrinos) have never been observed. Their existence would be theoretically motivated since quarks and leptons have right-handed components and they could explain the small neutrino masses, as described in Section 2.4.1. Right-handed neutrinos would interact only gravitationally and, for this reason, they are usually called sterile neutrinos. Even if not weakly-interacting, the eventual sterile neutrino(s) would still mix with the active flavours, affecting the measured oscillation patterns and providing an experimental detection signature. 
Another model for the neutrino masses would be the introduction of a Majorana mass term in the Lagrangian:

$$
-\mathcal{L}_{M}=\frac{1}{2} m_{M}\left(\bar{\nu}_{L} \mathcal{C} \bar{\nu}_{L}^{T}+\nu_{L} \mathcal{C} \nu_{L}^{T}\right)=\frac{1}{2} m_{M}\left(\bar{\nu}_{M} \nu_{M}\right)
$$

where the factor $\frac{1}{2}$ accounts for double-counting due to the hermitian conjugate being identical, $\nu_{M} \equiv \nu_{L}+\nu_{R}^{c}$ is the Majorana spinor, and $\mathcal{C}$ is the charge-conjugation operator. Here, the charge-conjugation operator must leave the field unchanged and the particle must then be neutral.

The only fermion that satisfies this requirement is the neutrino. If the neutrino is a Majorana particle, it will be its own antiparticle: in a CC interaction, the left-handed and right-handed components would produce a negative-charged and a positive-charged lepton, respectively.

An experimental evidence of the Majorana nature of the neutrinos would be the observation of the neutrinoless double $\beta$ decay $(0 \nu \beta \beta)$, where a $\nu_{e}$ is emitted and absorbed in the nucleus, producing two electrons and two protons in the final state. Figure 2.8 shows the Feynman diagram for a $0 \nu \beta \beta$ interaction.

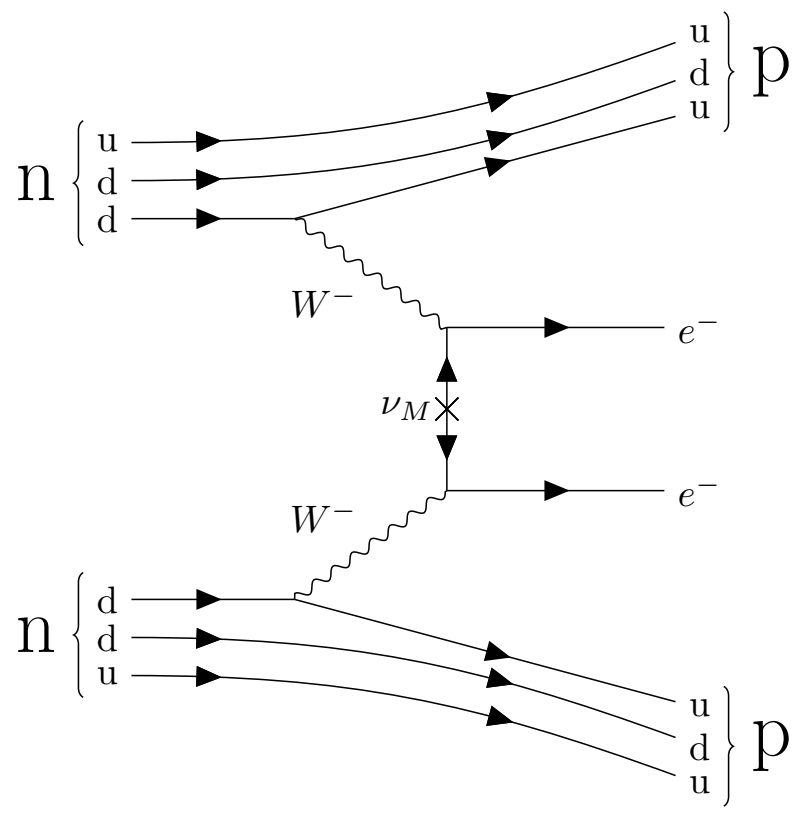

Figure 2.8: Feynman diagram for a neutrinoless double beta decay interaction, emitting two electron in the final state and no neutrinos. 
This process would also violate the lepton number with $\Delta L=2$. In this case, the PMNS matrix in Equation 2.11 acquires two extra Majorana phases in the form:

$$
U_{P M N S}^{M}=U_{P M N S}\left[\begin{array}{ccc}
e^{i \alpha} & 0 & 0 \\
0 & e^{i \beta} & 0 \\
0 & 0 & 1
\end{array}\right]
$$

Several experiments are actively looking or planning to look for $0 \nu \beta \beta$ decays, using ${ }^{130} \mathrm{Te}$ (CUORE 27, SNO+ 28), ${ }^{136}$ Xe (KamLAND-Zen 29, EXO 30, NEXT [31]), or ${ }^{76}$ Ge (GERDA [32]). The KamLAND-Zen collaboration measured the lower limit for $0 \nu \beta \beta$ decay half-life at $T_{\frac{1}{2}}>1.07 \times 10^{26}$ years, which corresponds to an effective Majorana mass of $61-165 \mathrm{meV}$ [29].

\subsubsection{The seesaw mechanism}

A process which could explain the small masses of the neutrinos, compared to the other fermions, is the so-called seesaw mechanism, which here we describe using the approach in 33. The most general Lagrangian with right-handed neutrinos can be written as:

$$
\begin{aligned}
-2 \mathcal{L}_{\text {mass }} & =\mathcal{L}_{L}^{D}+\mathcal{L}_{R}^{D}+\mathcal{L}_{L}^{M}+\mathcal{L}_{R}^{M}+\text { h.c. } \\
& =m_{D} \bar{\nu}_{R} \nu_{L}+m_{D} \bar{\nu}_{L}^{C} \nu_{R}^{C}+m_{L} \bar{\nu}_{L}^{C} \nu_{L}+m_{R} \bar{\nu}_{R}^{C} \nu_{R} .
\end{aligned}
$$

This term can be expressed as a matrix equation

$$
-2 \mathcal{L}_{\text {mass }}=\left[\begin{array}{ll}
\bar{\nu}_{L}^{C} & \bar{\nu}_{R}
\end{array}\right]\left[\begin{array}{ll}
m_{L} & m_{D} \\
m_{D} & m_{R}
\end{array}\right]\left[\begin{array}{c}
\nu_{L} \\
\nu_{R}^{C}
\end{array}\right],
$$

where $m_{D}$ is the Dirac mass term and $m_{L}\left(m_{R}\right)$ is the Majorana mass term for the left-handed (right-handed) component.

Since the SM forbids the left-handed Majorana term (it is not gauge invariant), it is possible to set $m_{L}=0$. For large values of $m_{R}$, the eigenvalues of the mass matrix are:

$$
\begin{aligned}
& m_{1}=\frac{m_{D}^{2}}{m_{R}} \\
& m_{2}=m_{R}\left(1+\frac{m_{D}^{2}}{m_{R}^{2}}\right) \approx m_{R} .
\end{aligned}
$$


With a value of $m_{R}$ at the GUT scale (around $10^{16} \mathrm{GeV}$ ), this would naturally give a small value for the neutrino masses.

\subsection{Neutrino interaction modes}

The interaction between the neutrino and the nucleon can have different forms and a good understanding of their phenomenology is of fundamental importance for the success of any neutrino experiment. Figure 2.9 shows the neutrino crosssection for charged-current interactions divided into three main interaction modes: quasi-elastic (QE), resonant (RES), and deep inelastic scattering (DIS), which we briefly characterise below.

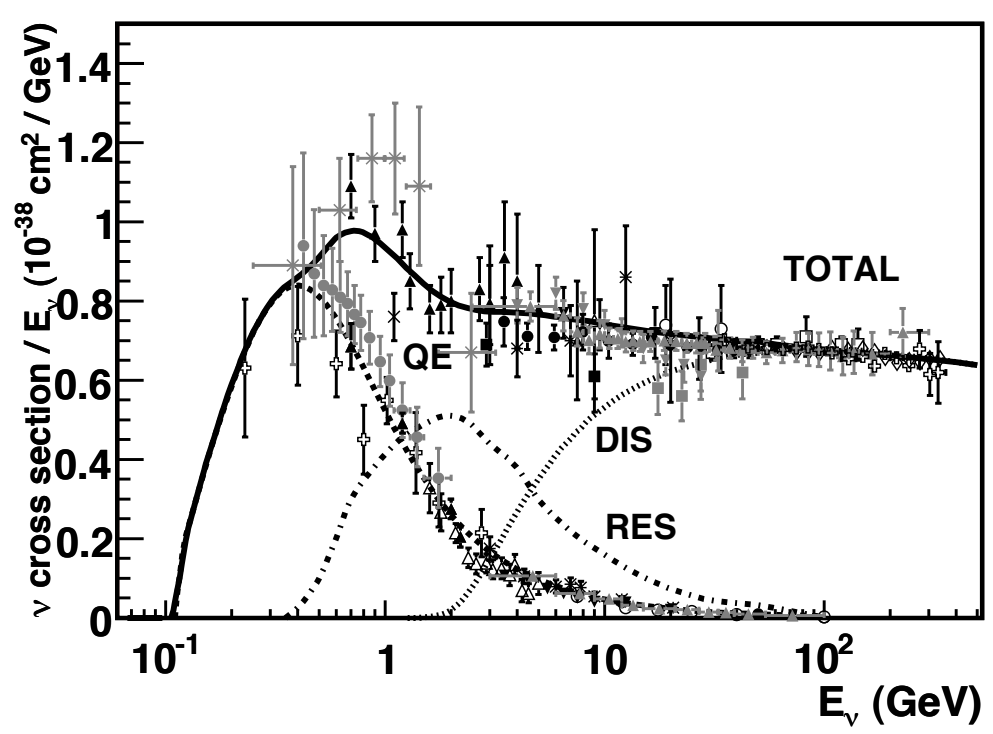

Figure 2.9: Total neutrino per nucleon charged-current cross sections (for an isoscalar target) divided by neutrino energy and plotted as a function of energy (from [34]).

Quasi-elastic interaction. In a charged-current quasi-elastic (CCQE) interaction, the neutrino exchange a $W$ boson with a single nucleon, which is knocked out and leaves a hole in the nucleus. Hence its name 1p-1h (one particle, one hole). In this case, the incident neutrino does not have enough energy to break up or excite the nucleus. This is the dominant interaction in the sub-GeV energy range. Figure 2.10 shows the Feynman diagram for a CCQE neutrino-nucleus interaction. 
The simulation used in this thesis employs the GENIE neutrino generator, set up with the Llewellyn-Smith parametrisation for the CCQE interactions [35 and the Relativistic Fermi Gas model for the nucleus 36. In this model, the interaction is calculated with the impulse approximation (IA) 37: the neutrino interacts with only one nucleon, which can have short-range correlations with other nucleons (bound but independent).

However, hadrons exiting the nucleus can re-interact and change identity or eject other hadrons (Final State Interactions, FSI), so it is possible to have a CCQE interaction with no protons or with a pion in the final state. It is also possible to have multinucleon excitations, mainly through the so-called meson exchange current (MEC) 38. This interaction is responsible for np-nh events, where more than one nucleon is emitted.

The neutral-current equivalent of a CCQE interaction is the neutral-current elastic scattering (NCE), which typically ejects a nucleon.

At low exchanged momentum $Q^{2}$ the neutrino can also scatter elastically with the entire nucleus (coherent elastic neutrino-nucleus) scattering, $\mathrm{CE} \nu \mathrm{NS}$ ), as recently detected by the COHERENT collaboration [39|. In this case, the nucleus remains in its initial state and its small recoil represents the only observable.

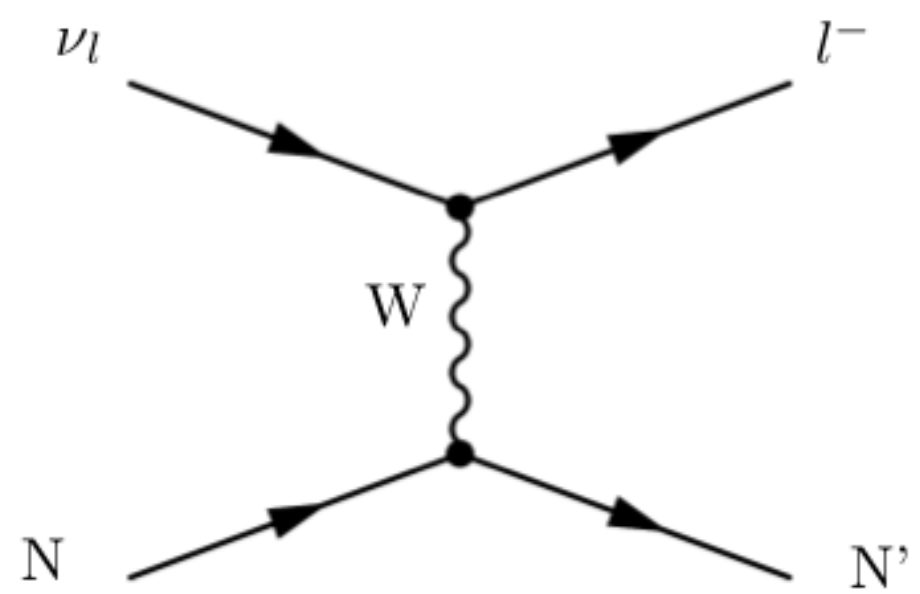

Figure 2.10: Feynman diagram of a charged-current quasi-elastic neutrino-nucleon interaction. 
Resonant and coherent interactions. In a resonant interaction, the neutrino has enough energy to excite the nucleon to form a resonance, which rapidly decays to a nucleon and one or more mesons while still in the nucleus, as in:

$$
\begin{aligned}
& \nu_{l}+p \rightarrow \Delta^{++} \rightarrow l^{-}+\pi^{+}+p \\
& \nu_{l}+n \rightarrow \Delta^{+} \rightarrow l^{-}+\pi^{+}+n .
\end{aligned}
$$

This interaction is allowed both by charged current and neutral current exchange.

The GENIE setup used here employs the Rein-Sehgal model [40] for the resonance production and the Bodek-Ritchie RFG model [38 for the interaction of the nucleon within the nucleus. Also in this case, the FSI can cause the absorption of the pion in the nucleus or exchange the charge of the pion.

At low exchanged momentum $Q^{2}$, pions can be produced also in coherent neutrino-nucleus processes, where the nucleus is left unchanged and a pion is emitted, as shown in the Feynman diagram of Figure 2.11b.

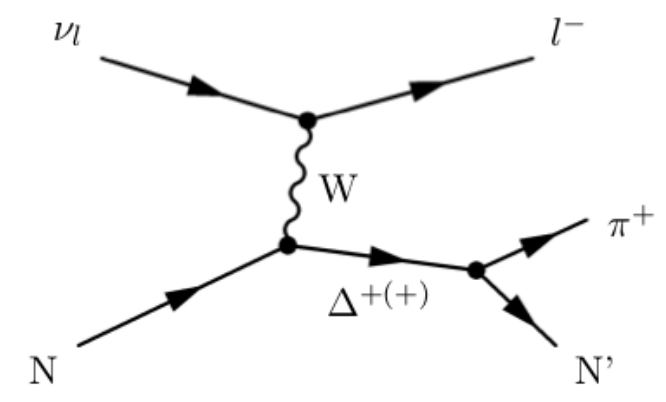

(a) Charged-current resonant interaction.

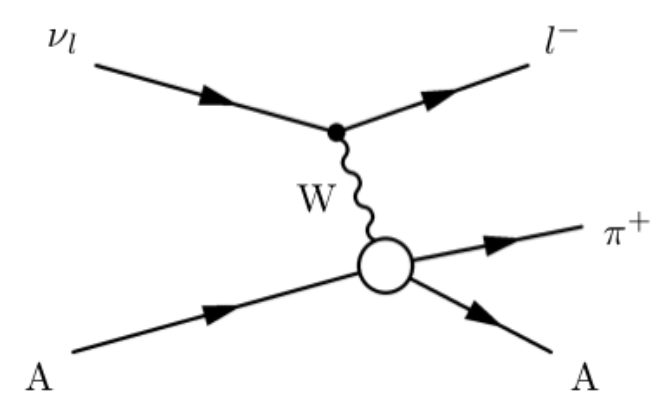

(b) Charged-current coherent interaction.

Figure 2.11: Feynman diagrams of two neutrino interactions which can produce pions in the final state, charged-current resonant (left) and charged-current coherent (right).

Deep inelastic scattering. In this case, the neutrino has enough energy to interact with the single nucleon components, the quarks, and to break up the nucleus. The result of this interaction often consists of several mesons in the final state. This is the dominant interaction mode for high-energy neutrinos $(>5 \mathrm{GeV})$. It is simulated by our setup of GENIE using the Bodek-Yang model 41. 
Figure 2.12 shows the Feynman diagram for this kind of interaction.

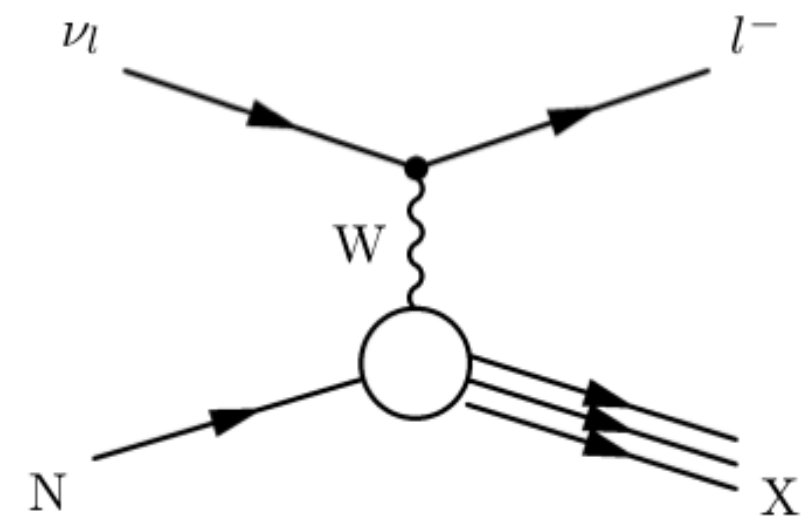

Figure 2.12: Feynman diagram of a charged-current deep inelastic scattering neutrinonucleon interaction.

This rich scenario makes the correct reconstruction of neutrino interactions very challenging for any experiment. Usually, oscillations experiments like MiniBooNE (see Section 3.2) look for CCQE interactions, whose signature in the detector is easier to identify, but which also require a precise assessment of the final-state interactions. The simulation of the nuclear behaviour after the neutrino interaction is very challenging and requires careful validation against hadronic observables, such as the number of reconstructed hadron tracks and the spectra of hadrons [42].

\subsection{Future research efforts}

Several experiments have shown compelling evidence for neutrino oscillations, which in turn require non-null neutrino masses. At the moment, massive neutrinos represent the only portal into BSM physics and, for this reason, neutrino physics is one of the most active sectors in particle physics. In the last decade, the parameters of the PMNS mixing matrix of neutrino flavours (see Equation 2.11) have been constrained to a precision smaller than $5 \%$, but some neutrino properties still need to be measured. In particular, the CP-violating phase, the neutrino mass hierarchy, and the Majorana or Dirac nature of the neutrino are all research topics actively investigated both by present and future experiments. 
The following chapters will focus on the search for a low-energy excess of electron neutrinos, which could be a hint of neutrino oscillation into a sterile state. 


\section{The LSND and MiniBooNE anomalies}

\section{Contents}

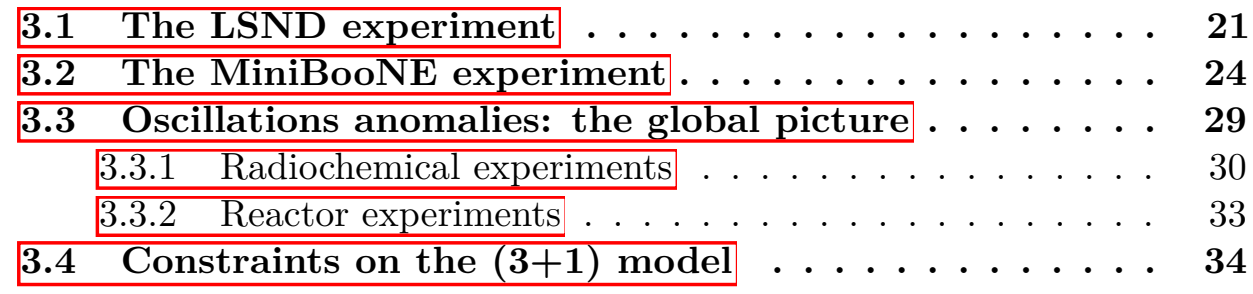

The LSND experiment observed an excess of $\bar{\nu}_{e}$ in a primarily $\bar{\nu}_{\mu}$ beam in 2001 . The MiniBooNE experiment, built to confirm or rule out the anomaly, observed a significant excess of $\nu_{e}$-like $\left(\bar{\nu}_{e^{-}}\right.$like $)$events in a primarily $\nu_{\mu}\left(\bar{\nu}_{\mu}\right)$ beam. Several other neutrino experiments have shown results not fully compatible with the three-flavour scenario and they will be briefly described. A summary of the global picture of the short-baseline anomalies will also be provided.

\subsection{The LSND experiment}

The Liquid Scintillator Neutrino Detector (LSND) was an experiment at the Los Alamos National Laboratory which aimed to detect $\bar{\nu}_{e}$ interactions in a mainly $\bar{\nu}_{\mu}$ beam. The neutrino beam was produced by firing an $800 \mathrm{MeV}$ proton beam into a target, producing charged pions, which were stopped in a beam dump. The $\pi^{-}$ 
part was electromagnetically captured by the nucleus, while the $\pi^{+}$component initiated the decay chain:

$$
\begin{aligned}
\pi^{+} \rightarrow & \mu^{+} \nu_{\mu} \\
& \quad\left\llcorner\mu^{+} \rightarrow e^{+} \bar{\nu}_{\mu} \nu_{e} .\right.
\end{aligned}
$$

Kinematically, it is possible to distinguish between the neutrino beam produced by decays at rest (DAR) of pions and muons, and the neutrino beam produced by decays in flight (DIF) of pions and muons. In LSND, this was achieved by looking at events with energies above (below) $60 \mathrm{MeV}$ to select the DIF (DAR) beam, since the maximum energy for a $\bar{\nu}_{\mu}$ produced by a stopping muon is $52.8 \mathrm{MeV}\left(m_{\mu} / 2\right)$.

The detector was filled with $167 \mathrm{t}$ of mineral oil $\left(\mathrm{CH}_{2}\right)$ and doped with $0.031 \mathrm{~g} / \mathrm{l}$ of organic scintillation material (butyl-PBD).

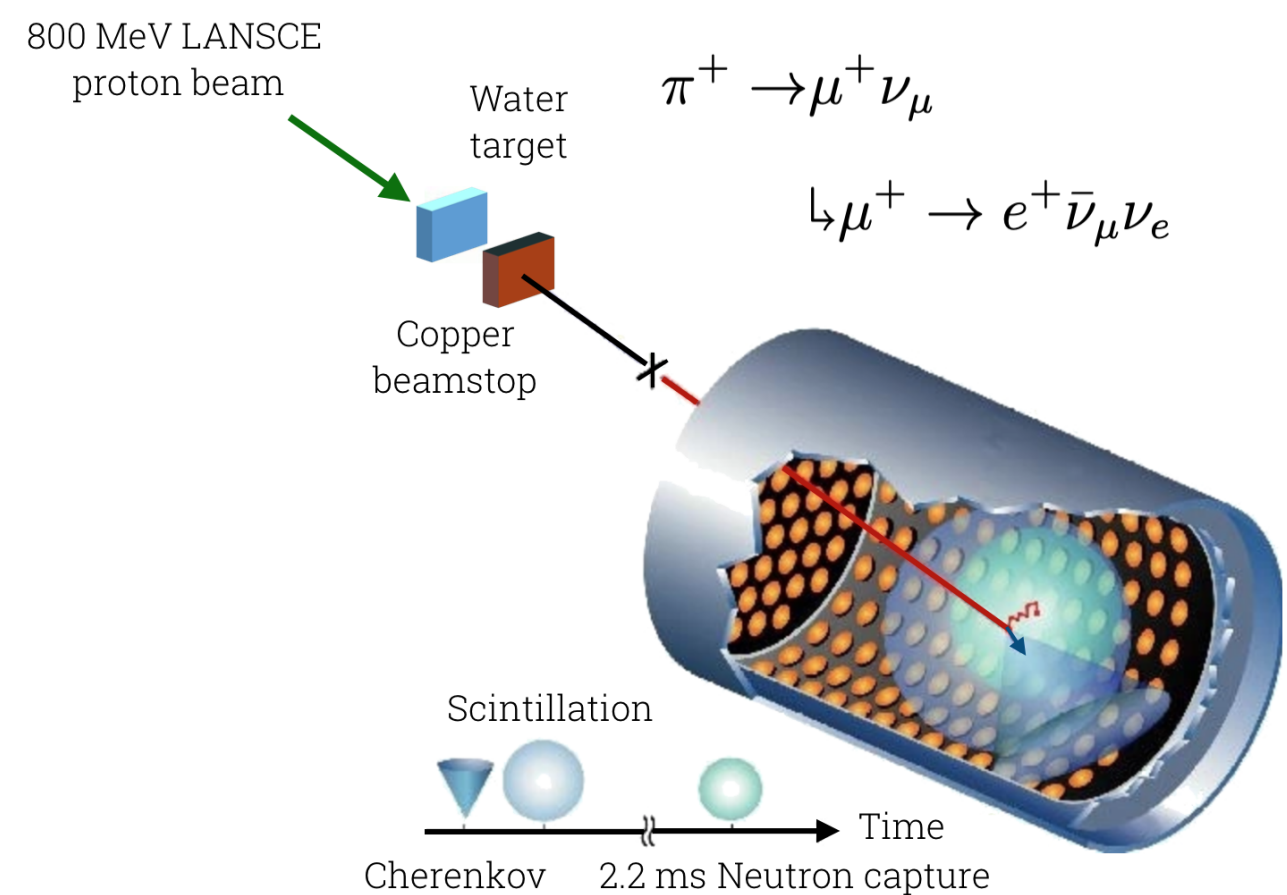

Figure 3.1: A schematic of the LSND experiment and its detection technique: the inverse $\beta$-decay of the neutrinos in the detector produce Cherenkov and scintillation light, in delayed coincidence with the light emitted by the neutron capture.

The $\bar{\nu}_{e}$ interactions were detected via an inverse $\beta$-decay process and tagged with a delayed coincidence between the positron and the subsequent neutron capture, in 
a fashion similar to the Cowan and Reines experiment. A schematic of the LSND experiment and its detection technique is shown in Figure 3.1.

LSND found an excess of $\bar{\nu}_{e}$ interactions in the DAR $\bar{\nu}_{\mu}$ beam with a significance of $3.8 \sigma$, which could be explained as $\bar{\nu}_{\mu}$ oscillating into $\bar{\nu}_{e}$ (Figure 3.2a). Given the $L / E \approx 0.75 \mathrm{~m} / \mathrm{MeV}$ of the experiment, the mass splitting term obtained with LSND data is $\Delta m_{\mathrm{LSND}}^{2} \approx 1 \mathrm{eV}^{2}$. This value is one order of magnitude larger than the mass splitting terms obtained with any other reactor, accelerator, atmospheric, or solar experiment 43 . An excess of $\nu_{e}$ was found also in the DIF $\nu_{\mu}$ beam, compatible with the $\bar{\nu}_{\mu} \rightarrow \bar{\nu}_{e}$ oscillation result [44. The comparison between the region allowed by LSND and the region allowed by other neutrino experiments in the $\left(\Delta m^{2}, \tan ^{2} \theta\right)$ parameter space is shown in Figure 3.7

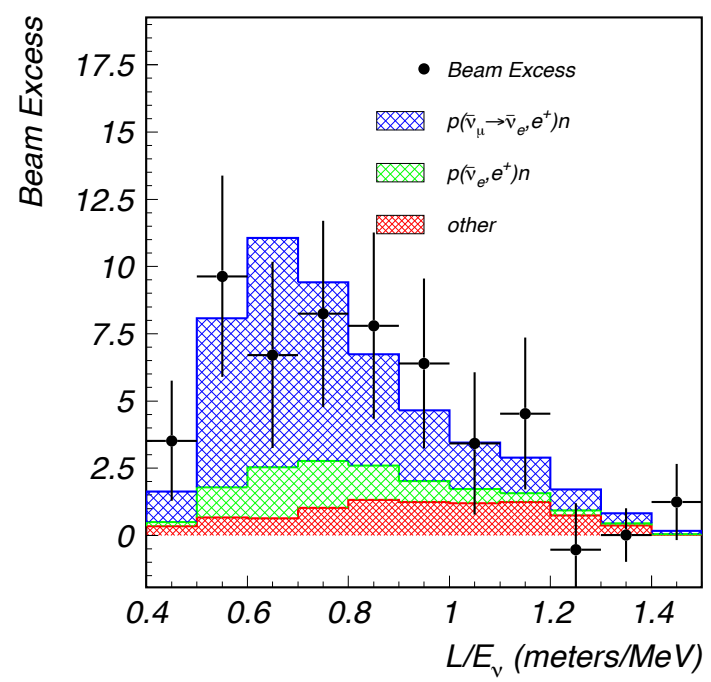

(a) $L / E_{\nu}$ distribution for the $\bar{\nu}_{e}$ events in the LSND experiment.

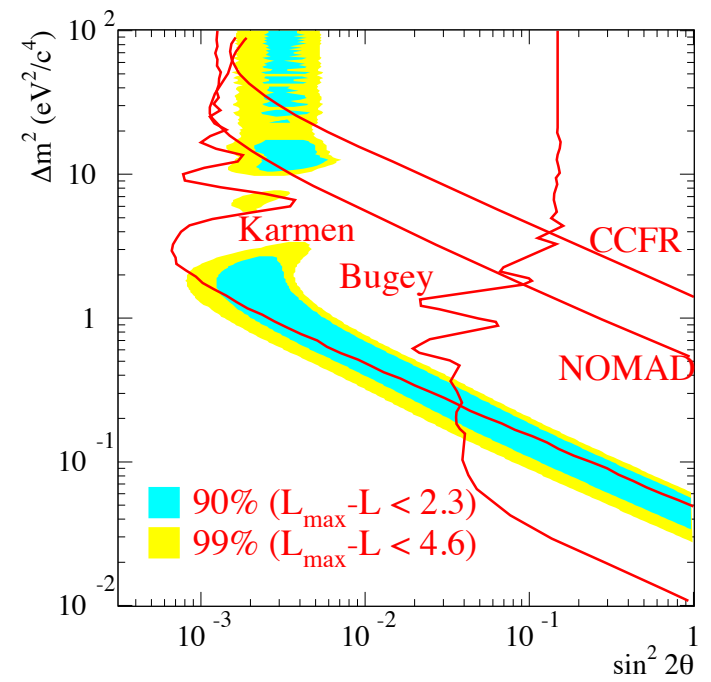

(b) Allowed and excluded regions in the $\left(\sin ^{2} 2 \theta, \Delta m^{2}\right)$ parameter space.

Figure 3.2: The excess of electron antineutrinos observed by the LSND experiment (left) can be interpreted with the presence of a fourth neutrino state. The mixing angles and mass splittings allowed by the LSND data are shown on the right at $90 \%$ C.L (blue) and 99\% C.L. (yellow), together with the 90\% C.L. exclusion limits from other experiments (solid red lines). From 43.

Figure $3.2 \mathrm{~b}$ shows the regions in the $\left(\sin ^{2} 2 \theta, \Delta m^{2}\right)$ parameter space allowed by the LSND data at 90\% CL and 99\% CL. The KARMEN experiment at the Rutherford Appleton Laboratory employed a setup similar to LSND in order to 
explore the same region, but it found no significant excess and ruled out a large subset of the LSND parameter space 45 .

\subsection{The MiniBooNE experiment}

The MiniBooNE experiment was designed to definitely test the LSND result. It consists of a spherical detector filled with mineral oil and located 541 meters downstream of the Booster Neutrino Beam (BNB) production target at Fermilab. This beam can run both in neutrino mode, producing a mainly $\nu_{\mu}$ beam, and in antineutrino mode, producing a mainly $\bar{\nu}_{\mu}$ beam. The BNB neutrino flux is described in detail by the MiniBooNE collaboration in [46] and will be summarised in Section 4.3. The beam energy is one order of magnitude larger than LSND (8 $\mathrm{GeV}$ vs. $800 \mathrm{MeV}$ ), but the two experiments have a comparable $L / E_{\nu}$ ratio.

The detector is equipped with 1280 8-inch photomultiplier tubes (PMTs) and employs a separated outer veto region with an extra 240 PMTs for cosmic-ray rejection. Particles interacting in the mineral oil produce Cherenkov light, if above production threshold. The particle identification is based on the different light patterns that each particle produces in the detector: in particular, high-penetrating, heavy particles such as muons will produce sharp rings of Cherenkov light, while lighter particles like electrons and photons will produce fuzzier rings. A neutral pion will instead produce two fuzzy rings partially overlapping when it decays to two photons $\left(\pi^{0} \rightarrow \gamma \gamma\right)$. This technique introduces an irreducible ambiguity in the final-state particles, since it is not possible to distinguish a single photon from an electron. Figure 3.3 shows three MiniBooNE event displays with a muon, an electron (or photon), and a $\pi^{0} \rightarrow \gamma \gamma$ decay in the final state.

Energy calibration at MiniBooNE was performed with in situ measurements. Cosmic muons, detected with an external hodoscope and stopping in the mineral oil, produce the typical Michel electron spectrum peaked at $m_{\mu} / 2=52.8 \mathrm{MeV}$. The invariant mass of $\pi^{0}$ decays can also be reconstructed to measure the energy response around $135 \mathrm{MeV}$. 


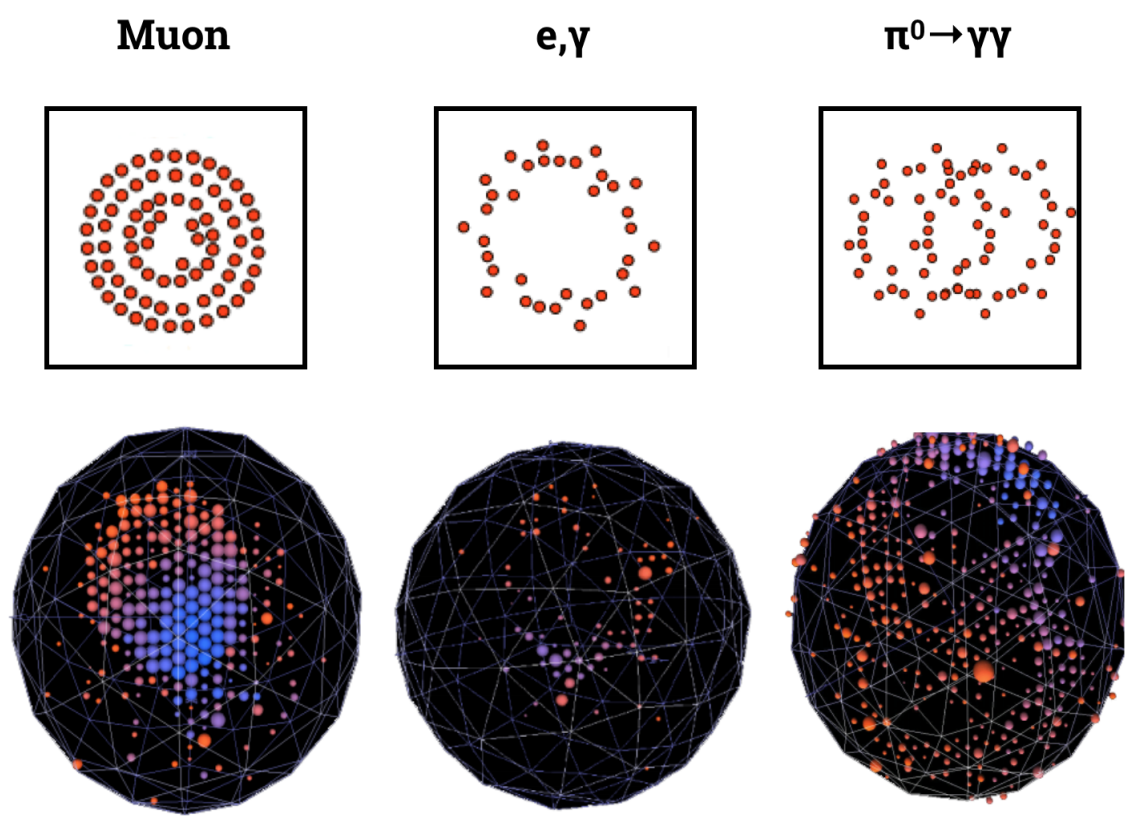

Figure 3.3: Schematics and event display for three topologies in the MiniBooNE detector.

The oscillation analysis of the MiniBooNE experiment looked for $\nu_{e}$ chargedcurrent quasi-elastic (CCQE) interactions, where the $\nu_{e}$ exchanges a charged $W$ boson with a neutron in the nucleus, producing an outgoing electron and a proton. This is the dominant interaction type in the sub-GeV region, as shown in Figure 2.9

However, as outlined in Section 2.5. FSI can alter the particles produced in the interaction and then detected by the apparatus. In particular, $\mathrm{CC} 1 \pi$ events with pion absorption represent a source of uncertainty for a CCQE analysis, since they share the same particles in the final state.

For this reason, in MiniBooNE, the selected events are called CCQE-like, since this definition relies only on the particles in the final state 47 .

The energy of the CCQE-like interaction $E_{\nu}^{Q E}$ is determined by the electron scattering angle $\theta$ and energy $E_{e}$, assuming the nucleon at rest, as:

$$
E_{\nu}^{Q E}=\frac{2 m_{n} E_{e}+m_{p}^{2}-m_{n}^{2}-m_{e}^{2}}{2\left(m_{n}-E_{e}+\cos \theta \sqrt{E_{e}^{2}-m_{e}^{2}}\right)},
$$

where $m_{n}, m_{p}$, and $m_{e}$ are the mass of the neutron, the proton, and the electron, respectively. In reality, the nucleon will have a Fermi momentum, which will smear out the energy measured with Equation 3.3. In this case, the estimation of the 
neutrino energy will depend on the particular model of Fermi motion employed in the simulation, introducing a systematic uncertainty in the measurement.

The presence of events with pion absorption or where the pion escapes the detector introduces as well a distortion in the energy distribution, since the approximation of a 2-body interaction of Equation 3.3 is no longer valid.

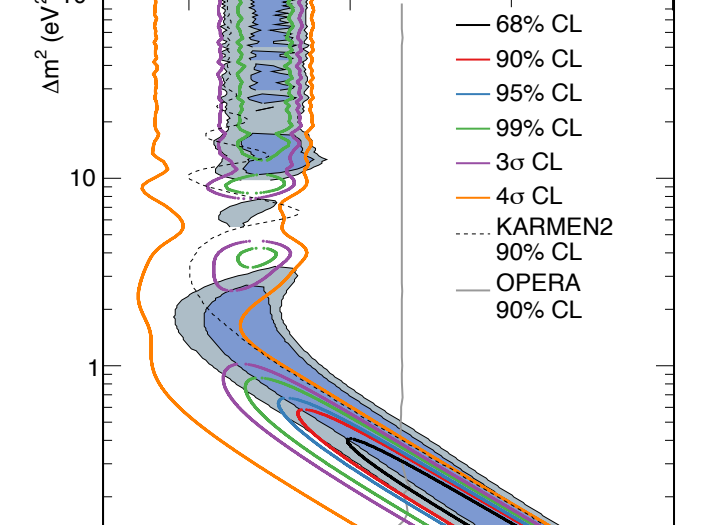

(a) $E_{\nu}^{Q E}$ spectrum in neutrino mode.

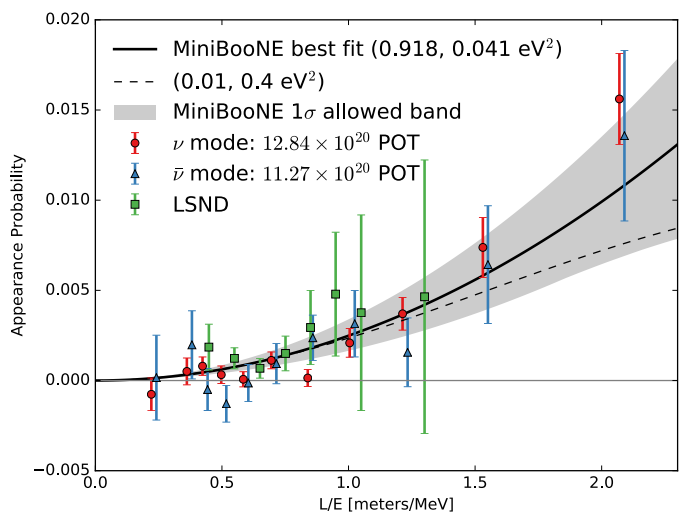

(b) Appearance probability.

Figure 3.4: The MiniBooNE neutrino mode corresponding to the total $12.84 \times 10^{20}$ POT data, for $\nu_{e}$ CCQE data (points with statistical errors) and background (histogram with systematic errors). The dashed line represent the two-neutrino model best fit (left). The appearance probability as a function of the $L / E$ ratio is in agreement with LSND data (right). Adapted from 48 .

The most recent result by the MiniBooNE collaboration [48 shows a $4.7 \sigma$ excess in the combined $\nu_{e}$ and $\bar{\nu}_{e}$ analysis, for $12.84 \times 10^{20}\left(11.27 \times 10^{20}\right)$ POT collected in neutrino (antineutrino) mode. The observed excess of data events is 460.5 \pm 99.0 . The energy spectrum of the $\nu_{E}^{\mathrm{CCQE}}$ selected events in neutrino mode is shown in Figure 3.4a. The analysis followed a blind approach, where the data sub-sample containing the signal events, defined requiring a single isolated electron in the detector, was not opened until the analysis tools and the simulation were well understood.

The $\nu_{e}$ oscillation was measured with a combined fit of the $\nu_{e}$ and $\nu_{\mu}$ selected events. This approach, which will be employed also by the MicroBooNE experiment, allows to measure more precisely the neutrino flux: in this way, the $\nu_{e}$ candidates from $\nu_{\mu}$ oscillation cannot be interpreted as an underestimation of the total neutrino flux, since this would show up as a disagreement in the number of $\nu_{\mu}$ events as well. 
A measurement of the $\nu_{\mu}$ component of the flux allows also to partially constrain the number of intrinsic $\nu_{e}$ since around half of them are produced in the decay:

$$
\begin{aligned}
\pi^{+} \rightarrow & \mu^{+} \nu_{\mu} \\
& \quad\left\llcorner\mu^{+} \rightarrow e^{+} \bar{\nu}_{\mu} \nu_{e} .\right.
\end{aligned}
$$

The excess of data events is in the sub-GeV energy region and is consistent in energy and magnitude with the LSND result. The two excess combined give a significance of $6.0 \sigma$. Figure $3.4 \mathrm{~b}$ shows the agreement of the appearance probability as a function of the $L / E_{\nu}$ distribution for LSND and MiniBooNE.

\section{MiniBooNE backgrounds}

The background events of the MiniBooNE experiment can be divided into four main categories:

Intrinsic $\nu_{e}$. The $\nu_{e}$ component of the beam, coming from $\mu^{ \pm}, K^{ \pm}$, and $K^{0}$, is the irreducible background of the experiment, since it can't be distinguished from $\nu_{\mu}$ oscillating into $\nu_{e}$. This component of the flux is partially constrained by measuring the $\nu_{\mu}$ interactions.

Misidentified $\pi^{0}$. The background from misidentified $\pi^{0}$ events represents the largest component. These events are particularly challenging to reconstruct since very forward-boosted photons will appear in the detector as a single fuzzy ring. The MiniBooNE collaboration has constrained this contribution by reconstructing the invariant $\pi^{0}$ mass of the event and obtaining a sample with a purity $>90 \%$ of NC $\pi^{0}$ events. The total uncertainty on the NC background is $7 \%$.

Misidentified $\Delta \rightarrow N \gamma$. A neutral current resonant interaction can produce a $\Delta$ resonance, which has a rare electromagnetic decay channel $\Delta \rightarrow N \gamma$, where $N=n, p$. This channel is also constrained by the $\mathrm{NC} \pi^{0}$ in situ measurement, times the small branching ratio $(0.56 \pm 0.04) \%$. The uncertainty on this component is $12 \%$. 
Dirt. The dirt background, meaning neutrino interactions happening outside the detector but where at least one particle in the final states interacts inside, is also constrained with an in situ measurement, where events reconstructed close to the detector boundaries and pointing inwards are selected.

Both the $\pi^{0}$ and the $\Delta \rightarrow N \gamma$ backgrounds come from the inability of a Cherenkov detector to distinguish between photons and electrons, which is instead one of the most powerful capabilities of a LArTPC, as it will be described in detail in Section 4.4. MiniBooNE is also not able to distinguish between negative and positive charged particles, making it impossible to distinguish between neutrino and antineutrino events.

Non-beam backgrounds (such as cosmic rays) are removed by requiring light in the detector in time with the BNB spill of $1.6 \mu$ s and no activity in the outer veto volume, achieving a $99.99 \%$ rejection efficiency. In order to reject the beamrelated background events, the MiniBooNE collaboration employed an electron-muon likelihood cut, an electron-pion likelihood cut, and a cut on the invariant $m_{\gamma \gamma}$ mass.

The average selection efficiency for $\nu_{e}^{\mathrm{CCQE}}$ events is $\sim 20 \%$ 48.

\section{Possible interpretations of the LSND and MiniBooNE re- sults}

A proposed solution to the LSND anomaly is to have one sterile additional neutrino state, which would mix with the standard three neutrinos, in a $(3+1)$ scenario. The PMNS matrix, in this case, will have an extra dimension $(4 \times 4)$ and the sterile mass neutrino eigenstate will be written as:

$$
\left|\nu_{s}\right\rangle=\sum_{\alpha}^{3+1} U_{i, \alpha}\left|\nu_{\alpha}\right\rangle
$$

A diagram of the mass hierarchy in this scenario is available in Figure 3.5.

As shown in Figure 3.4b, the LSND excess seems to be in agreement with the results of the MiniBooNE experiment, both in neutrino and antineutrino mode. The MiniBooNE collaboration was able to constrain all the simulated experimental backgrounds with in situ measurements. The excess must then come from an 


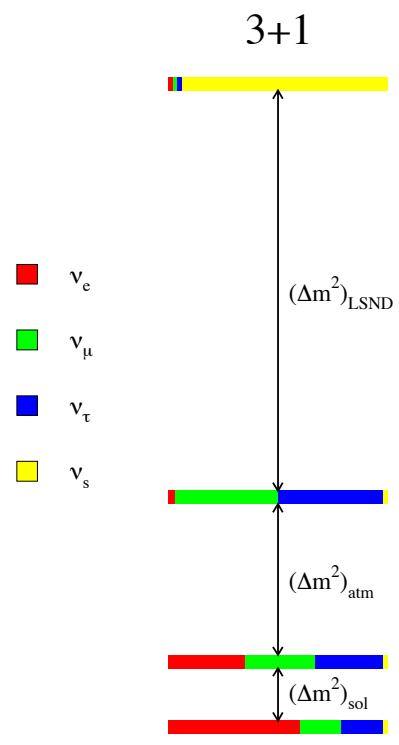

Figure 3.5: Neutrino mass normal hierarchy in the scenario of $3+1$ neutrinos (from $[6]$ ).

unexpected background source or from BSM interactions, such as the existence of one or more sterile neutrinos. Figure 3.6 shows the MiniBooNE allowed regions in neutrino and antineutrino mode for the two-neutrino oscillation model. The best-fit point, however, is disfavoured by the OPERA $\nu_{e}$ appearance analysis at $90 \%$ C.L. 50 .

\subsection{Oscillations anomalies: the global picture}

The global fit of neutrino oscillations experiments in the 2-neutrino approximation (so using Equations 2.8 and 2.9 is shown in Figure 3.7. Atmospheric, solar, and reactor experiments roughly overlap in three regions in the $\left(\tan ^{2} \theta, \Delta m^{2}\right)$ space, giving three mixing angles and two mass splittings values, as expected in a 3-flavour scenario.

However, LSND and MiniBooNE results do not agree with the allowed regions since their mass splitting term, at the same $L / E$, is much larger $\left(\Delta m^{2} \approx 1 \mathrm{eV}^{2}\right)$. Moreover, they are not the only two experiments to have observed anomalies in the neutrino sector. Several other experiments obtained results not completely in agreement with the theoretical expectations. In particular, it is possible to identify two categories of anomalies, classified according to the experimental technique employed: 


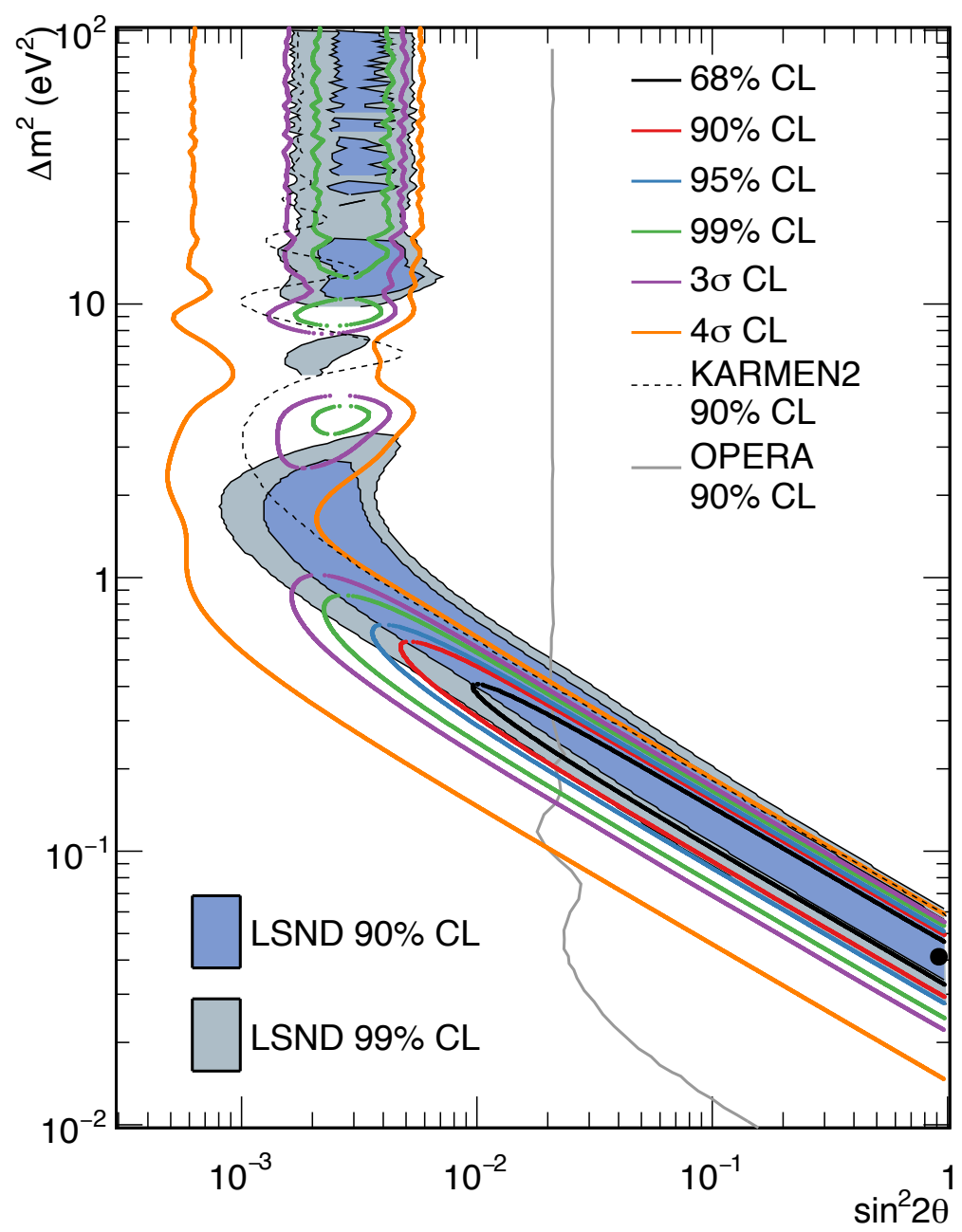

Figure 3.6: MiniBooNE allowed regions for the combined neutrino mode and antineutrino for events with $200<E_{\nu}^{Q E}<3000 \mathrm{MeV}$ within a two-neutrino oscillation model. The black point at $\left(\sin ^{2} 2 \theta, \Delta m^{2}\right)=\left(0.96,0.041 \mathrm{eV}^{2}\right)$ represents the best fit $[48]$.

inverse beta decay from solar neutrinos of gallium into germanium (radiochemical experiments) and inverse beta decay from reactor neutrinos (reactor experiments).

\subsubsection{Radiochemical experiments}

The GALLEX experiment at Gran Sasso and the SAGE experiment at Baksan employed a detection technique similar to the one of Ray Davis experiment at Homestake. In this case, solar neutrino interactions were detected through inverse $\beta$ decay of ${ }^{71} \mathrm{Ga}$ atoms into ${ }^{71} \mathrm{Ge}$ (instead of ${ }^{37} \mathrm{Cl}$ into ${ }^{37} \mathrm{Ar}$ at Homestake):

$$
\nu_{e}+{ }^{71} \mathrm{Ga} \rightarrow e^{-}+{ }^{71} \mathrm{Ge} .
$$




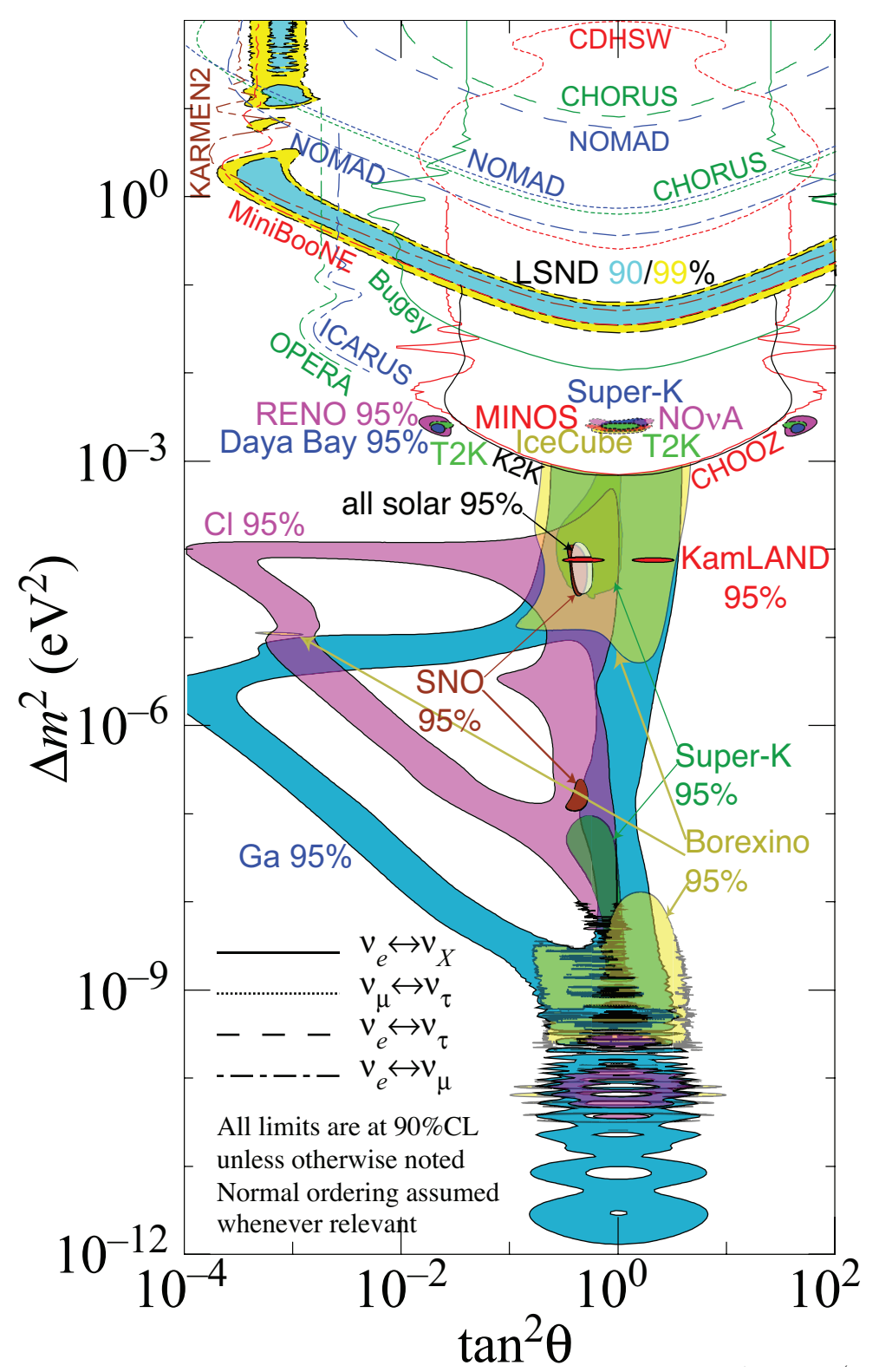

Figure 3.7: The squared-mass splittings and mixing angles favoured (solid regions) or excluded (open regions) by existing neutrino oscillation measurements. Results are categorised by channels: $\nu_{e}$ disappearance (solid lines), $\nu_{\mu} \leftrightarrow \nu_{\tau}$ (dotted lines), $\nu_{e} \leftrightarrow \nu_{\tau}$ (dashed lines), and $\nu_{e} \leftrightarrow \nu_{\mu}$ (dashed-dotted lines). The normal mass ordering is assumed where relevant. Taken from 19. Does not include MiniBooNE latest result 48 . 
The energy threshold for this reaction is $233 \mathrm{keV}$, which allows to observe the neutrino interactions produced in the solar $p p$ chain reaction (see Figure 2.4). Both experiments employed intense radioactive sources for calibration. GALLEX used a ${ }^{51} \mathrm{Cr}$ source, while SAGE used ${ }^{51} \mathrm{Cr}$ and ${ }^{37} \mathrm{Ar}$. These two sources decay via electron capture, emitting an electron neutrino:

$$
{ }_{Z}^{A} \mathrm{X}+e^{-} \rightarrow{ }_{Z-1}^{A} \mathrm{Y}+\nu_{e}
$$

The two experiments observed a deficit of $\nu_{e}$ interactions in all the three cases, which favours with $2.7 \sigma$ significance the hypothesis of short-baseline neutrino oscillation 51 .

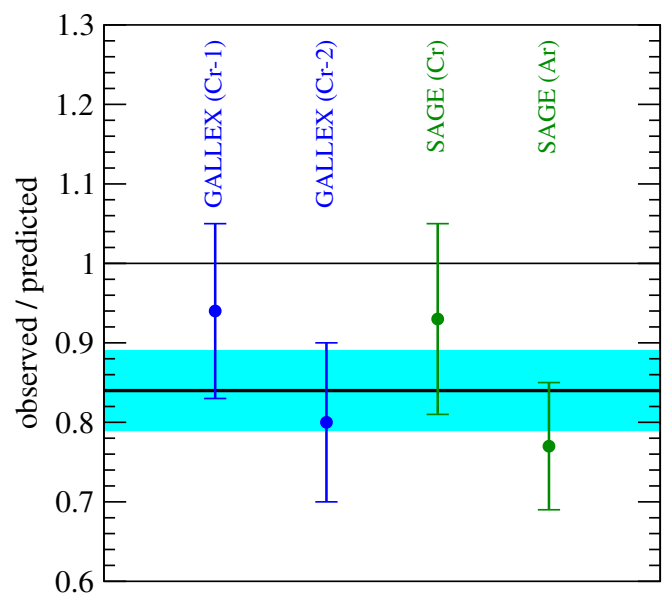

(a) Observed / predicted ratio of $\nu_{e}$ interactions.

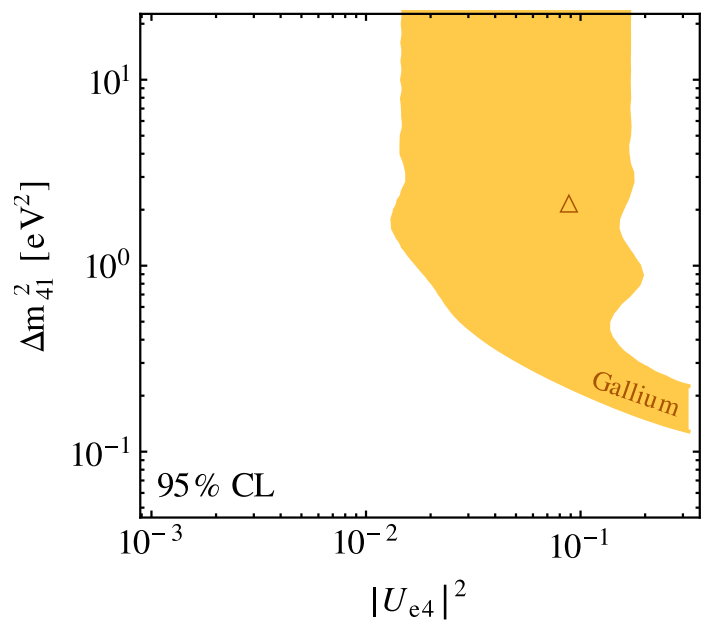

(b) Allowed parameter space for sterile neutrino oscillation.

Figure 3.8: The SAGE and GALLEX experiments observed a deficit of electron neutrino interactions using radioactive isotopes, which could be explained by introducing oscillations into a sterile neutrino state.

Figure 3.8 shows the deficit for the four calibration runs (two with ${ }^{51} \mathrm{Cr}$ for GALLEX, one with ${ }^{51} \mathrm{Cr}$ and one with ${ }^{37} \mathrm{Ar}$ for SAGE) and the allowed parameter space in the case of sterile neutrino oscillations in the $(3+1)$ model.

Curiously, during the second data-taking run of SAGE, 2 tons of gallium were stolen from the detector (3.6\% of the total mass) 52 . 


\subsubsection{Reactor experiments}

Several reactor neutrino experiments have measured a deficit of events in the antineutrino spectra. This anomaly first appeared in 2011, when an improved calculation of the reactor antineutrino spectra was made available 53 . Historical data from several reactor experiments, which before the recalculation were in agreement with the theoretical predictions, all showed a $\sim 6 \%$ deficit in the spectra, as shown in Figure 3.9 .

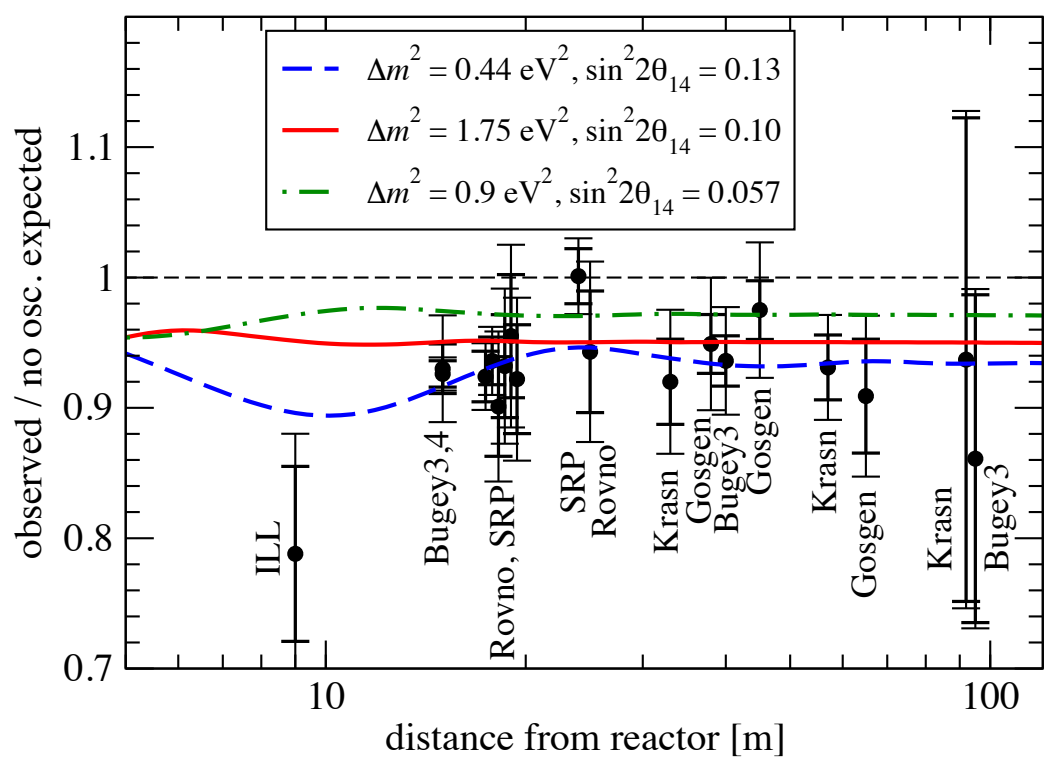

Figure 3.9: Fraction between observed and predicted $\bar{\nu}_{e}$ flux at several reactor neutrino experiments. Several models for sterile neutrino oscillation for different mass splitting terms and mixing angles are also shown.

This anomaly was first confirmed by a blind analysis of the Daya Bay collaboration 54 and then observed also by the RENO and Double Chooz detectors. More recently, these three experiments have also observed an excess of events (bump) around $5 \mathrm{MeV}$. In order to clarify the nature of the flux deficit, the Daya Bay experiment was able to correlate the antineutrino flux with the fuel composition in the reactor. The fuel evolves with time: the main fissile component, ${ }^{235} \mathrm{U}$, gets smaller, while the ${ }^{239} \mathrm{Pu}$ increases. The model used to predict the inverse beta-decay yield is $3.1 \sigma$ in disagreement with data 55 . If the deficit is caused by sterile neutrinos, then it should not depend on the fissile material and the sterile neutrino 
hypothesis cannot be used to explain the fuel evolution model discrepancy. A combined analysis of Daya Bay and NEOS data hints to excess production of ${ }^{235} \mathrm{U}$ as an explanation for the $5 \mathrm{MeV}$ bump $[56$.

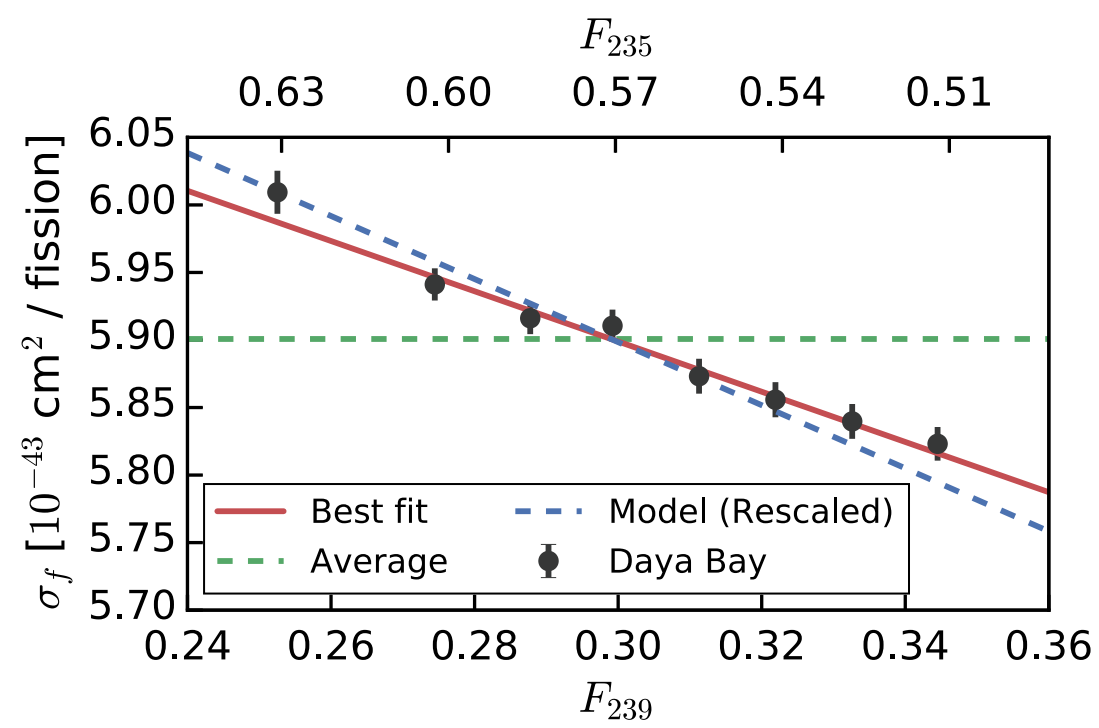

Figure 3.10: Inverse $\beta$-decay yield per fission, $\sigma_{f}$, versus effective ${ }^{239} \mathrm{Pu}$ (lower axis) or ${ }^{235} \mathrm{U}$ (upper axis) fission fraction. From $[55]$.

A new generation of reactor neutrino experiments should definitely solve the reactor anomaly. In particular, experiments like PROSPECT [57], SoLid [58], and STEREO [59] are placed very close to small fission coresmade almost entirely of ${ }^{235} \mathrm{U}$ and they are sensitive to eventual short-baseline sterile neutrino oscillations.

\subsection{Constraints on the $(3+1)$ model}

In the presence of a sterile neutrino, its mixing with the active flavours would affect the $\nu_{e}$ appearance, the $\nu_{e}$ disappearance, and the $\nu_{\mu}$ disappearance probabilities. However, the combined analysis of MINOS, Daya Bay, and Bugey-3 $\nu_{\mu}$ disappearance data 60 also excludes the best-fit point. A recent result from IceCube 61 further restricts the available parameter space, leaving little room for the $(3+1)$ hypothesis. The tension emerges both by comparing appearance and disappearance experiment (Figure 3.11a) and by comparing $\nu_{e}$ data $\left(\nu_{e} \rightarrow \nu_{e}, \nu_{e} \rightarrow \nu_{\mu}\right)$ and $\nu_{\mu}$ data $\left(\nu_{\mu} \rightarrow \nu_{\mu}\right)$ (Figure 3.11b). A global analysis 62 excludes the $(3+1)$ model at $4.7 \sigma$ level. 


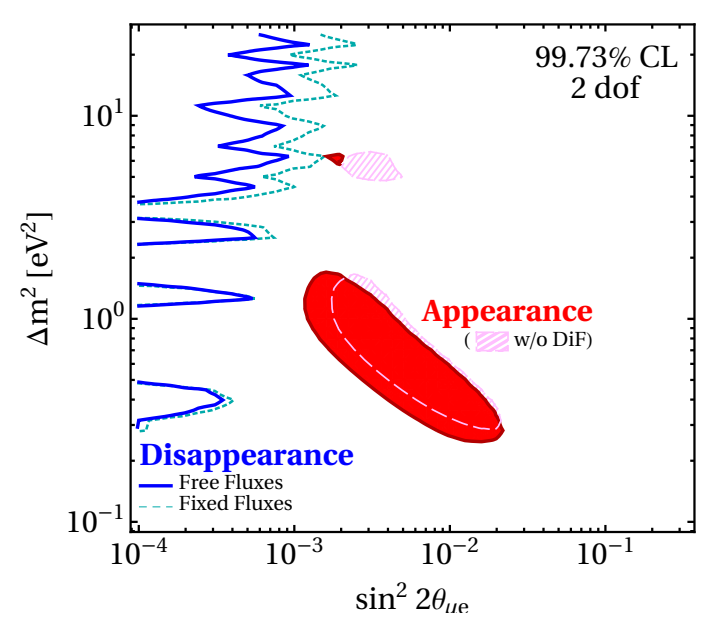

(a) Comparison between regions allowed by appearance results (filled red region) and regions excluded by disappearance results (solid blue line).

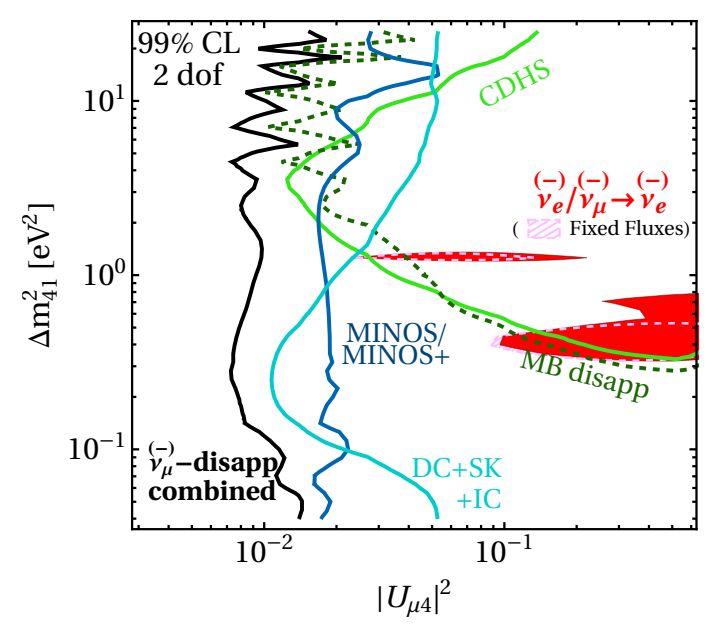

(b) Comparison between regions allowed by $\nu_{e}$ data $\left(\nu_{e} \rightarrow \nu_{e}\right.$ and $\nu_{e} \rightarrow \nu_{\mu}$, filled red region) and regions excluded by $\nu_{\mu}$ disappearance data (solid lines).

Figure 3.11: There is a severe tension between appearance and disappearance results within the $(3+1)$ model. The free (fixed) fluxes lines of the plots on the left refer to the constraining (or not) of the reactor fluxes in the fits. Adapted from 62 .

Other explanations have then been proposed for the excess: $3+N$ sterile neutrinos with $N>1$ [63], CPT violation [64], and resonant neutrino oscillations 65 among the others. The explanation of the excess with the presence of a new particle decaying or scattering in the detector is severely constrained by kinematic arguments 66 .

In summary, after the definitive confirmation of neutrino oscillations, achieved by employing different detection techniques, a series of new experiments collected data not fully compatible with a three-flavour scenario. In particular, a combined analysis of the LSND and MiniBooNE data gives a $6.0 \sigma$ significance for an excess of electron-like events. This result is however in tension with other experiments if interpreted as the oscillation into a sterile neutrino state.

Other experiments have also performed measurements not fully in agreement with the theoretical expectations, using both reactor antineutrinos and neutrinos from radioactive isotopes, but a coherent explanation for these anomalies still has to be provided. 


\section{4 \\ The MicroBooNE experiment}

\section{Contents}

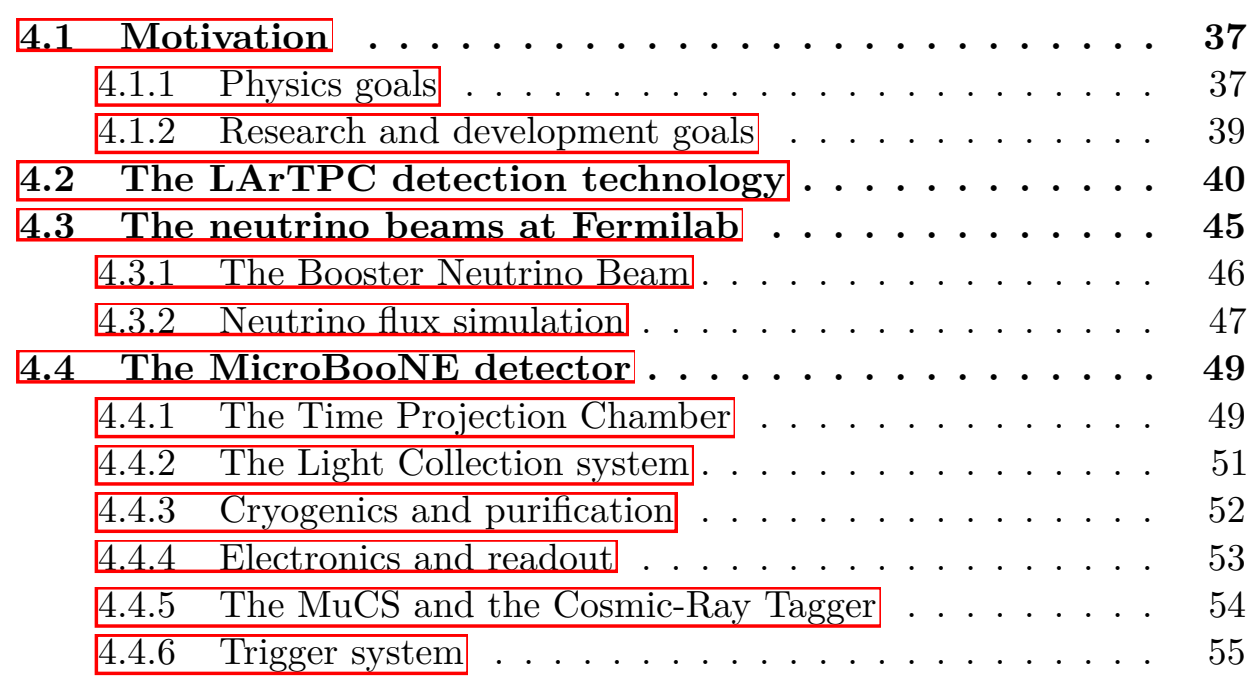

This chapter presents an overview of the MicroBooNE experiment, with a focus on the Liquid Argon Time Projection Chamber technology. A description of the MicroBooNE detector is essential in order to understand the analysis described in the following chapters. The selection efficiency of low-energy electron neutrino events and the rejection of background events can directly depend on the detector properties. The analysis is also affected by systematic uncertainties in the detector simulation, which will be partially addressed here. A brief description of the 
MicroBooNE neutrino beam will also be provided.

\subsection{Motivation}

The MicroBooNE (Micro Booster Neutrino Experiment) experiment is an $89 \mathrm{t}$ active volume Liquid Argon Time Projection Chamber (LArTPC) located at the Fermi National Accelerator Laboratory (FNAL) in Batavia, IL and on-axis with the Booster Neutrino Beam (BNB). The experiment was designed to study shortbaseline neutrino oscillations and neutrino-argon cross section. It is the largest neutrino LArTPC detector currently active in the world. This technology offers very high spatial resolution and calorimetric capabilities, which allow for detailed tracking, vertexing, and particle identification.

\subsubsection{Physics goals}

As described in Section 3.2. MiniBooNE, being a Cherenkov detector, is not able to distinguish between single photons and electrons in the final state. As such, it is not possible to determine the nature of the excess of low-energy events. If the excess is caused by photons, one explanation could be provided by an underestimation of one of the background components. An electron nature of the excess, instead, could be a strong hint for BSM physics.

\section{Addressing the MiniBooNE anomaly}

The MicroBooNE experiment was designed to definitely clarify the MiniBooNE anomaly since the LArTPC technology allows for powerful electron/photon separation. One way to achieve this goal is to measure the spatial gap between the neutrino interaction vertex and the start of the electromagnetic shower. An electron will start producing a ionising trail immediately, whereas a photon will usually leave a visible gap, due to the radiation length in liquid argon $X_{0}=14 \mathrm{~cm}$. A second way to distinguish between electrons and photons is to measure the energy loss per distance travelled $(d E / d x)$ of the electromagnetic shower produced in the liquid argon. For electrons above $100 \mathrm{MeV}$, the theoretical expectation of the most 
probable $d E / d x$ is around $2 \mathrm{MeV} / \mathrm{cm}$, while the photon will have a most probable value approximately twice as large, due to the pair-production process $\gamma \rightarrow e^{+} e^{-}$ 67. In order to clarify MiniBooNE result, MicroBooNE is then performing two parallel analyses, one assuming that the excess is caused by photon production in the electromagnetic $\Delta \rightarrow N \gamma$ decay and one assuming that the excess is caused by electron interactions. In this way, we will be able to definitely identify the nature of the excess and check if it is caused by electron neutrino interactions or by an underestimation of one of the backgrounds. The calorimetric and spatial-resolution capabilities will allow having a sensitivity to the excess similar to MiniBooNE, while having an active detector mass five times smaller. The search for electron-like low-energy interactions will be described in detail in the following chapters.

\section{Cross-section measurements}

MicroBooNE will also provide precise neutrino-nucleon cross-section measurements. The neutrino interactions in the energy range of the BNB span from quasi-elastic to deep inelastic scattering, making it possible to explore several nuclear effects and complex topologies. In particular, it is possible to measure the pion production in neutral current (NC) and charged current (CC) interactions. MicroBooNE should be able to solve the tension between the large $\mathrm{NC} \pi^{0}$ component reported by MiniBooNE 68 and the small CC $\pi^{+}$component reported by SciBooNE 69 and $\mathrm{K} 2 \mathrm{~K}[70$. A precise measurement of the photon production will also help to constrain the $\Delta \rightarrow N \gamma$ background, important for the MiniBooNE low-energy excess analysis.

The measurement of neutrino cross sections in liquid argon is also of fundamental importance for the design of the largest next-generation neutrino experiment, DUNE (Deep Underground Neutrino Experiment). Its current proposed design includes a 40kton LArTPC as far detector, two orders of magnitude larger than MicroBooNE 71 .

\section{Supernova and exotic searches}

The MicroBooNE detector is located just below the surface level and is constantly bombarded by cosmic rays, which interact in the liquid argon leaving ionisation trails. This background, together with the small active volume of the detector 
(compared with large-scale water Cherenkov experiments) can limit the capabilities of certain physics analyses, such as proton decay searches. However, MicroBooNE can study the interaction of charged kaons in the liquid argon, which can represent a background to future proton decay searches in DUNE. The existence of proton decay is predicted by several Grand Unification Theories (GUTs) and a lower proton lifetime of $5.9 \times 10^{33}$ years was set by the Super-Kamiokande experiment looking at the $p \rightarrow \nu K^{+}$channel $[72]$. The kaon decay chain $K \rightarrow \pi \rightarrow \mu \rightarrow e$ represents also an important benchmark for the reconstruction capabilities of the LArTPC.

The MicroBooNE detector is suited to detect neutrinos produced by a supernova $(\mathrm{SN})$ in the Milky Way or in its immediate surroundings, which would result in around $30 \mathrm{CC} \nu_{e}$ interactions with electron energy above $10 \mathrm{MeV}$. However, being constantly hit by cosmic rays, the detector cannot directly trigger on SN neutrinos and it relies on the SuperNova Early Warning System (SNEWS) 73 to record an eventual SN event.

\subsubsection{Research and development goals}

MicroBooNE is currently the largest active neutrino LArTPC in the world, which makes it the first experiment to precisely assess the automated neutrino reconstruction capabilities of this technology. The detection of neutrinos by a large-scale LArTPC was pioneered by the ICARUS collaboration, which designed and assembled the ICARUS T600 detector 74. The detector consists of two LArTPC modules with a total active mass of $476 \mathrm{t}$, around five times larger than MicroBooNE. However, it was employed as a long-baseline neutrino detector on the CNGS (CERN Neutrinos to Gran Sasso) beam and it detected only four $\nu_{e}$ interactions 75 , compared with the several hundred expected at MicroBooNE. It was recently refurbished and moved to Fermilab to be placed on the BNB, as a part of the future Short Baseline Neutrino (SBN) program. On a smaller scale, the ArgoNeuT experiment operated a $0.25 \mathrm{t}$ TPC on the NuMI neutrino beam at Fermilab 76 and measured for the first time the neutrino cross section on argon atoms. 
The ambitious physics program of the DUNE experiment, whose ultimate goal is to verify the presence of $\mathrm{CP}$ violation in the leptonic sector, requires a very good understanding of the technology: in particular, the effect of high electric field on long distances, of the ions recombination in the LAr, and of the ions absorption by the impurities must be precisely quantified. MicroBooNE was also the first large-scale LArTPC to have part of the electronics chain operating directly in the liquid argon (cold electronics), allowing for higher signal-to-noise ratio. An overview of the MicroBooNE detector will be provided in Section 4.4. A small-scale prototype of the DUNE detector, ProtoDUNE, was recently built and commissioned at CERN, with an active mass of 450 t. The ProtoDUNE detector, however, runs on a test-beam line and will not detect neutrino interactions.

\subsection{The LArTPC detection technology}

The concept of a Liquid Argon Time Projection Chamber was first laid out by Willis and Radeka in 1974 [77 and then adapted by Rubbia in 1977 as a detector for neutrino interactions 78 .

Generally speaking, a Time Projection Chamber is a volume with a constant electric field applied between two of its sides, the anode (the positive plane) and the cathode (the negative plane). It is possible to fill the volume with a non-conductive material such as inert gases or liquids.

A charged particle traversing the medium inside the TPC will ionise the material: a trail of ionisation electrons will be produced in correspondence with the path of the charged particle. The constant electric field will transport the electrons towards the anode with a constant drift velocity, preserving their topological and calorimetric information and appearing as a projection of the particle trajectory on the anode plane. The distance between the anode and the interaction point will be given by the time the ionisation electron takes to reach the anode. Figure 4.1 shows the operating principle of a TPC in the case of the MicroBooNE detector.

As the name says, a LArTPC is a TPC filled with liquid argon. This material provides several advantages, which made this technology particularly suitable for 


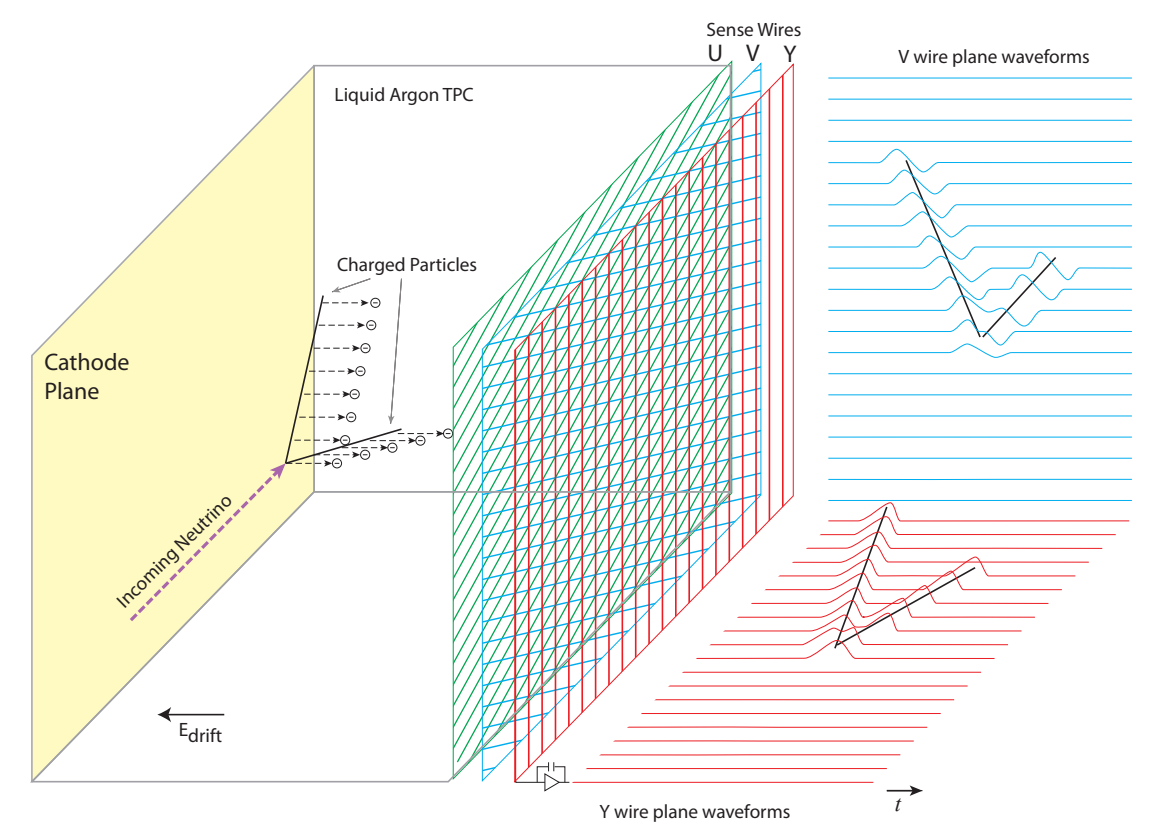

Figure 4.1: Diagram of the operating principle of the MicroBooNE LArTPC, showing the waveforms produced by the ionisation trails in the collection plane (red) and in one induction plane (blue).

neutrino detection. Among the main ones we can enumerate (1) its substantial density $\left(1.4 \mathrm{~g} / \mathrm{cm}^{3}\right.$ at $\left.87.3 \mathrm{~K}\right)$, which allows to have a detectable amount of neutrino interactions, (2) its high stability, being a noble gas, and (3) its natural abundance (1\% of the atmosphere), which makes the LArTPC technology highly scalable and affordable.

However, the relatively slow drift velocity of the electrons in the liquid argon causes the typical read-out of a large-scale LArTPC to be in the order of the milliseconds (which corresponds to the time a ionisation electron takes to travel from the cathode to the anode), making this technology sub-optimal for highrate experiments.

Another fundamental property of the argon, which makes it particularly suitable for high-energy physics experiments, is that it produces scintillation light when excited, being at the same time transparent to the wavelength of this scintillation light, described below. In this way, a detector on surface such as MicroBooNE can collect the light (in our case with photomultipliers placed inside the LAr) and 
trigger the TPC readout in coincidence with the neutrino beam, suppressing the background caused by cosmic rays outside the beam time window.

A LArTPC can achieve a very high spatial resolution, similar to the one of the bubble chambers, allowing at the same time for the digitisation of the signal. For this reason, it has often been called a fully electronic bubble chamber 79 .

\section{Light production}

The scintillation light is produced when an atom of argon in the ground state shares an electron with one argon of atom in an excited state, forming an $\mathrm{Ar}_{2}$ excimer. When the excimer decays and a $128 \mathrm{~nm}$ photon is emitted, the two argon atoms are both left in the ground state. This small wavelength is typically difficult to detect with standard photomultipliers. In the MicroBooNE experiment, the PMT plates are coated with tetraphenyl butadiene (TPB), which acts as wavelength shifter. The time distribution of the scintillation light emission has two characteristics components at $6 \mathrm{~ns}$ and $1.5 \mathrm{\mu s}$.

The liquid argon has also a high light yield, comparable to the one of scintillating crystals, with $4 \times 10^{4} \gamma / \mathrm{MeV}$. The amount of light, however, can be quenched by the presence of nitrogen impurities in the argon, which, in the case of MicroBooNE, are kept below the 2 ppm level.

\section{Ionisation electrons}

The work function for ionising an argon atom is $W_{\text {ion }}=23.6 \mathrm{eV}$, which means that a charged particle in the $\mathrm{MeV}$ range will leave a ionisation trail of tens of thousands of electrons. Under an electric field, these free electrons will travel towards the anode with a constant drift velocity, but during their path they can undergo several attenuation processes, which decrease the actual number of electrons reaching the wire plane.

In particular, the electrons can recombine with ionised $\mathrm{Ar}^{+}$atoms: this recombination effect is usually the main contributor to the signal attenuation. This effect depends on the $d E / d x$ of the particle, on the electric field in the liquid argon, and on the angle of the ionisation particle with respect to the electric field. The ICARUS 
experiment measured the dependence of the recombination as a function of the $d E / d x$ and the applied electric field [80], as shown in Figure 4.2. The ArgoNeuT experiment found the recombination effect in a LArTPC to be well described both by the Birks' model 81$]$ and by a modified version of the Box model 82 .

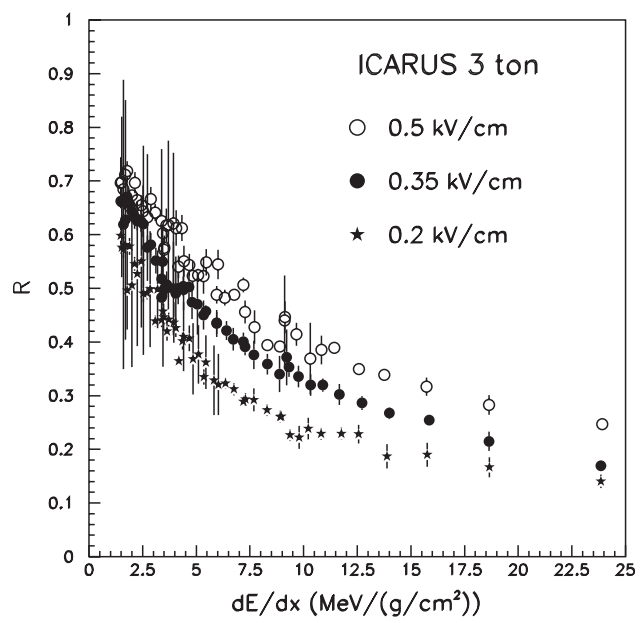

(a) Recombination factor as a function of the particle $d E / d x$.

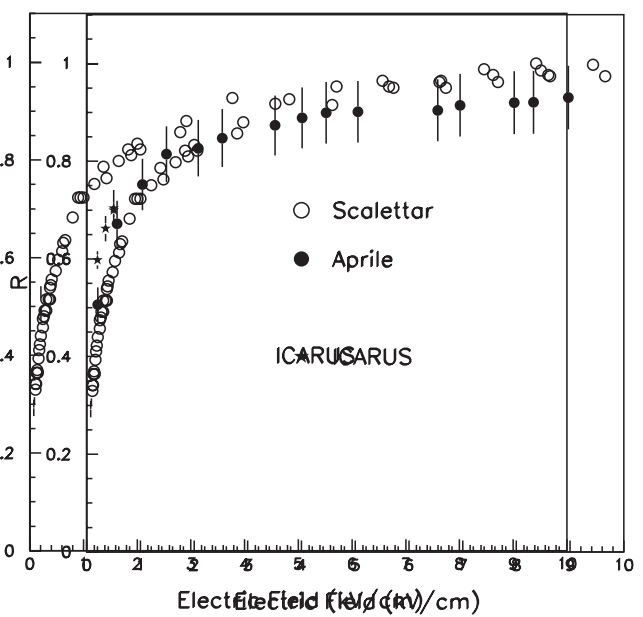

(b) Recombination factor as a function of the electric field in the detector.

Figure 4.2: The ICARUS collaboration measured the recombination factor $R$, defined as the ratio between the measured and the theoretical stopping power, as a function of the $d E / d x$ (left) and of the electric field (right). From 80 .

The transport of the electrons in the LArTPC is affected by diffusion, caused by their thermal velocity, which spreads out the ionisation electrons while they travel towards the anode. It is a three-dimensional effect, usually separated into its longitudinal and transverse components.

LArTPCs placed on surface, such as MicroBooNE, are also constantly hit by an intense flux of cosmic rays, which will produce several ionisation trails. These trails produce ionisation electrons and positive $\mathrm{Ar}^{+}$, with the ions slowly moving towards the cathode until they will recombine with a free electron. The build-up of $\mathrm{Ar}^{+}$ ions leads to a distortion of the electric field within the detector, defined as spacecharge effect (SCE). The SCE causes a displacement in the reconstructed position of ionisation electrons, as well as variations in the amount of charge quenching experienced by ionisation throughout the volume of the TPC. In MicroBooNE, the electric field distortion can be as high as $15 \%$ and was measured using a 
sample of cosmic rays triggered by a small cosmic-ray counter (briefly described in Section 4.4.5 83. The spatial distortions at the top and the bottom of the LArTPC are shown in Figure 4.3 .

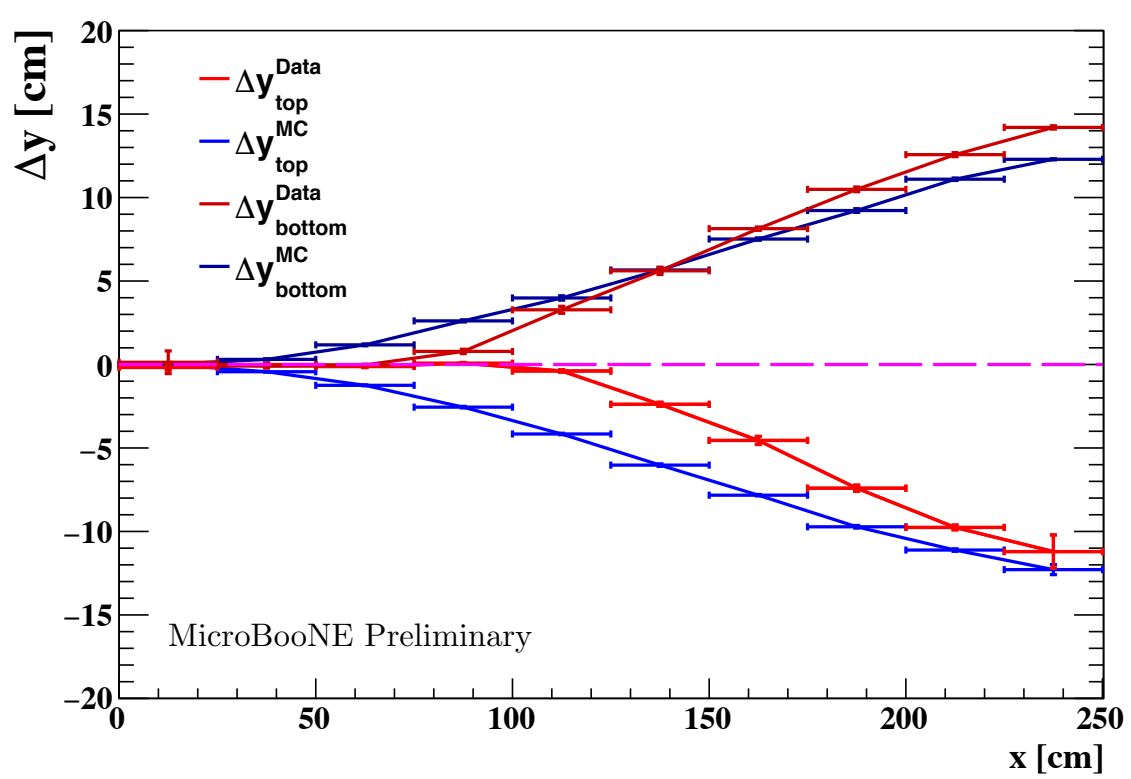

Figure 4.3: Predicted $\left(\Delta y^{\mathrm{MC}}\right)$ and measured $\left(\Delta y^{\text {Data }}\right)$ space-charge distortions as a function of the drift coordinate at both the top and bottom of the TPC. The discrepancy between data and Monte Carlo is caused by the absence of the liquid argon flow in the simulation. Error bars are statistical only. From 83 .

The presence of impurities in the liquid argon, such as oxygen, nitrogen, and water can also attenuate the signal, absorbing the ionisation electrons during their path in the liquid argon. The amount of drifting electrons decline as a function of the distance from the wire plane, since the electrons need to travel a longer path. The attenuation is well modelled by an inverse exponential function and the decay time constant is called electron lifetime. MicroBooNE purification system, described in Section 4.4, achieved an $\mathrm{O}_{2}$ contamination smaller than 100 ppt and an electron lifetime larger than $18 \mathrm{~ms}$ 84. In MicroBooNE, the electron lifetime was estimated by measuring the ratio between the charge at the anode and the charge at the cathode with crossing cosmic muons, through the relation:

$$
\frac{Q_{A}}{Q_{C}}=\exp \left(-t_{\mathrm{drift}} / \tau\right)
$$


where $t_{\text {drift }}$ is the drift time $(2.3 \mathrm{~ms})$ and $\tau$ is the electron lifetime. Figure 4.4 shows the variation of the $Q_{A} / Q_{C}$ ratio over time in the MicroBooNE LArTPC.

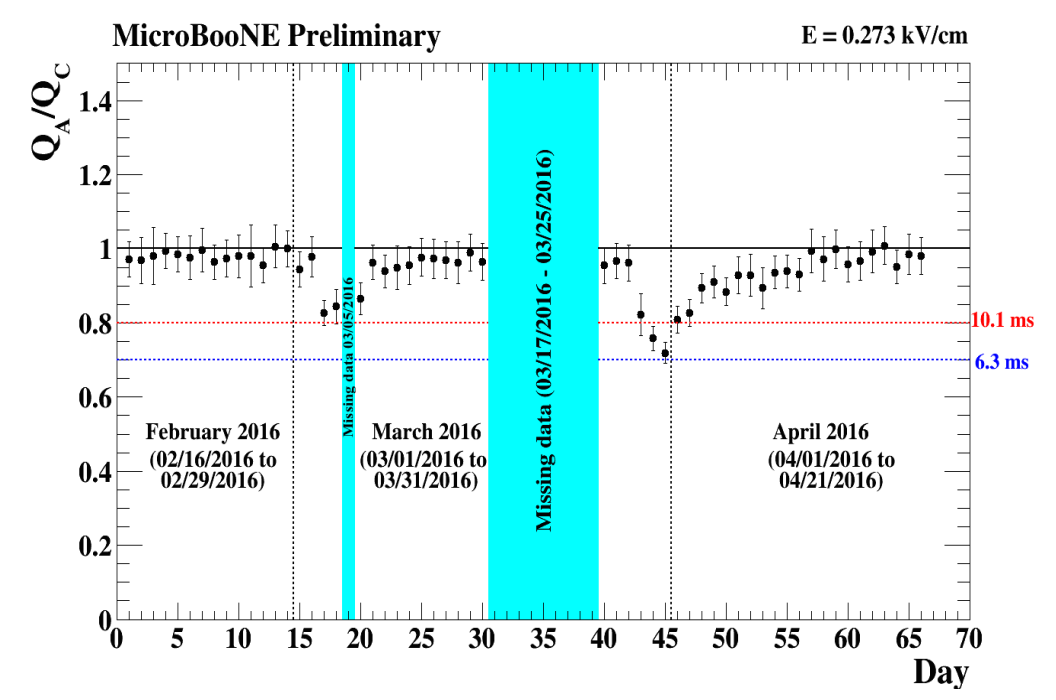

Figure 4.4: Variation of the $Q_{A} / Q_{C}$ charge ratio between February and April 2016 in the MicroBooNE LArTPC. From [84.

\subsection{The neutrino beams at Fermilab}

The use of an artificial neutrino source presents two main advantages: (1) the neutrino energy spectrum and its flavour components can be precisely characterised and tuned for the specific physics goals, and (2) the position of the detector can be optimised to observe the oscillation peak (or dip). The design of a neutrino beam generally follows the same pattern: it starts with a proton beam, which hits a target and produces hadrons. These hadrons are focused by a magnetic horn into an empty pipe, where they decay, producing the neutrinos. The neutrino beam then travels through the ground and hits one or more neutrino detectors.

At Fermilab, there are two neutrino beams currently active: the Booster Neutrino Beam (BNB) and the Neutrinos from the Main Injector (NuMI) beam, with different energy, flavour composition, and direction. MicroBooNE is placed on-axis with the BNB and $470 \mathrm{~m}$ far from the target. While it is also able to detect off-axis neutrinos from the NuMI beam, the analysis presented in this document will focus on the neutrinos coming from the BNB. 
The accelerator chain starts with a source of hydrogen gas, which is ionised and accelerated by an empty cavity with $-35 \mathrm{kV}$ voltage. This $\mathrm{H}^{-}$stream is focused by two solenoids and transformed into a pulsed beam $100 \mu$ s long at $15 \mathrm{~Hz}$. The Radio Frequency Quadrupole (RFQ) then accelerates the beam to an energy of $750 \mathrm{keV}$. The $\mathrm{H}^{-}$beam is then fed to the Linear Accelerator (LINAC), where two series of RF cavities bring it from $750 \mathrm{keV}$ to $400 \mathrm{MeV}$. The negative hydrogen ions are stripped of their electrons by a carbon foil and the proton beam is finally injected into the Booster synchrotron. Here, after several thousand laps, the proton beam reaches their maximum kinetic energy in the Booster of $8 \mathrm{GeV}$.

The $8 \mathrm{GeV}$ proton batch is $1.6 \mu \mathrm{s}$ long and divided into 84 bunches 2 ns wide. From the Booster, the proton batch can be extracted to the Booster Neutrino target or be injected into the Main Injector, where it is accelerated up to $120 \mathrm{GeV}$.

\subsubsection{The Booster Neutrino Beam}

The BNB is the main neutrino beam for the MiniBooNE and MicroBooNE experiments and it will be used also for the future Short Baseline Neutrino program 85 . It was designed mainly for the MiniBooNE experiment and, in order to suppress the background coming from resonant and DIS interactions (as described in Section 2.5), its neutrino flux is peaked below $1 \mathrm{GeV}$.

The BNB target is made of a beryllium disk $0.51 \mathrm{~cm}$ in radius and $71.1 \mathrm{~cm} \mathrm{long}$, which corresponds to 1.7 interaction lengths. This material minimises the radiative losses due to its relatively low density. The number of protons hitting the target (protons-on-target, POT) is measured by two toroids placed upstream the target within a $2 \%$ uncertainty. Each BNB bunch usually delivers $4 \times 10^{12}$ POT.

The hadrons produced in the p-Be interactions are then focused by the electromagnetic horn. The horn is a pulsed toroidal electromagnet with a peak current of $170 \mathrm{kA}$ and a magnetic field at the centre of $1.5 \mathrm{~T}$. It focuses the secondary hadrons along the horn axis: depending on the direction of the current, the positive (negative) charged particles are focused (deflected), producing a neutrino (antineutrino) beam. 


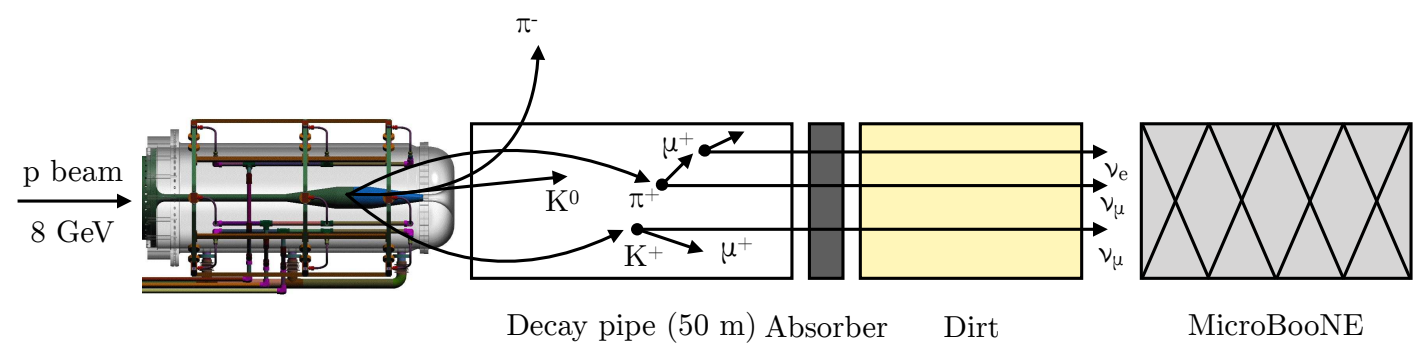

Figure 4.5: Schematic of the Booster Neutrino Beam chain in neutrino mode, showing, from left to right, the focusing horn, the decay pipe, the absorber, and the dirt. Dimensions are not to scale.

The hadrons with the right charge, mainly kaons and pions, travel through the decay pipe filled with air and are then stopped by a concrete absorber. The neutrinos produced in the decays travel through the ground and hit the detector, which in the case of MicroBooNE is placed $470 \mathrm{~m}$ far from the target. Figure 4.5 shows a schematic of the Booster Neutrino Beam stages in the neutrino mode.

The muon neutrinos come from the decay of the pions (and in turns of the muons) and the decay of the kaons. Muons and kaons are also responsible for the electron neutrino contamination in the beam. Figure 4.6 shows the flavour composition of the BNB flux in neutrino mode at the MicroBooNE location.

The pulsed structure of the neutrino beam is of fundamental importance for on-surface experiments like MiniBooNE and MicroBooNE. In order to suppress nonbeam related backgrounds (such as cosmic rays), the detector is triggered only in coincidence with the beam-gate window. In this way, MiniBooNE achieved a cosmicray background rejection of $99.987 \%$ [86. For a LArTPC such as MicroBooNE, the cosmic-ray rejection is more challenging, since the data acquisition window must be at least as long as the drift time window (so in the order of the milliseconds). The techniques employed to reject cosmic rays will be described in Section 6.2.

\subsubsection{Neutrino flux simulation}

The MiniBooNE collaboration developed a detailed simulation of the BNB flux, described in 46 . 


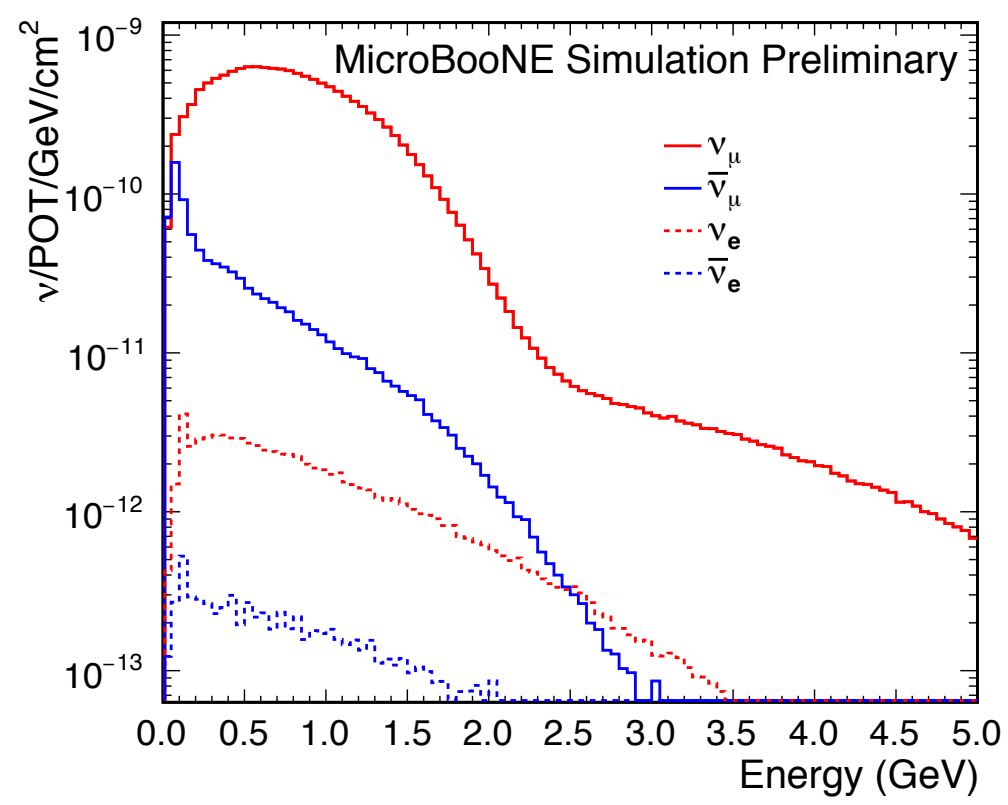

Figure 4.6: BNB absolute flux prediction in neutrino mode at the MicroBooNE location, break down by neutrino flavour.

Here, we will enumerate the main stages of the simulation, in order to clarify the flux systematic uncertainties assessment in Section 7;

Beamline geometry. The geometry of the beamline components is simulated in detail, including the beam horn, the target, and the decay pipe.

Proton generation. The number of protons delivered by the beam must be precisely estimated, accounting also for beam optics effects.

Interactions of protons with the target. This is the step with the largest uncertainty. The MiniBooNE collaboration estimated the $\pi^{+}$and $\pi^{-}$production using the data from the HARP experiment [87, while the $K^{+}$component was constrained using the result of the SciBooNE experiment 88 .

Propagation in the material. The interaction of the particles in the material is simulated with the GEANT toolkit 89 .

Particles decay into neutrinos. The branching ratios and the kinematic properties of the particles which produce the neutrino beam must be assessed with precision. 
Table 4.1 shows the uncertainties on the various components of the BNB flux for the MicroBooNE experiment. These uncertainties will be reflected in the flux systematic uncertainties of the analysis described in Section 7.

Table 4.1: Systematic uncertainties on the BNB flux calculation. The other category includes uncertainties in pion and nucleon cross-sections on beryllium and aluminium, as well as the horn current calibration uncertainty, and uncertainty in the horn current distribution.

\begin{tabular}{lllll}
\hline Systematic uncertainty & $\nu_{\mu} / \%$ & $\bar{\nu}_{\mu} / \%$ & $\nu_{e} / \%$ & $\bar{\nu}_{e} / \%$ \\
\hline Proton delivery & 2.0 & 2.0 & 2.0 & 2.0 \\
$\pi^{+}$ & 11.7 & 1.0 & 10.7 & 0.03 \\
$\pi^{-}$ & 0.0 & 11.6 & 0.0 & 3.0 \\
$K^{+}$ & 0.2 & 0.1 & 2.0 & 0.1 \\
$K^{-}$ & 0.0 & 0.4 & 0.0 & 3.0 \\
$K_{L}^{0}$ & 0.0 & 0.3 & 2.3 & 21.4 \\
Other & 3.9 & 6.6 & 3.2 & 5.3 \\
\hline Total & 12.5 & 13.5 & 11.7 & $22.6 \%$ \\
\hline
\end{tabular}

\subsection{The MicroBooNE detector}

The MicroBooNE detector consists of a rectangular LArTPC with dimensions of $256 \mathrm{~cm}($ width $) \times 233 \mathrm{~cm}$ (height) $\times 1037 \mathrm{~cm}$ (length) placed in a cylindrical cryostat. It sits on-axis with the BNB, $470 \mathrm{~m}$ far from the neutrino beam target. The mass of liquid argon in the active volume, defined as the portion of the argon encompassed by the TPC, is $89 \mathrm{t}$.

Figure 4.7 shows a 3D rendering of the cryostat containing the TPC. The main components of the detector (the TPC, the light collection system, the cryogenics, the cosmic-ray tagger, and the electronics and readout) are described in detail in 90 and will be summarised below.

\subsubsection{The Time Projection Chamber}

The TPC consists of a cathode, an anode, and a field cage. The cathode, made of a plane of 9 stainless steel sheets $2.3 \mathrm{~mm}$ thick, operates at a voltage of $-70 \mathrm{kV}$. 


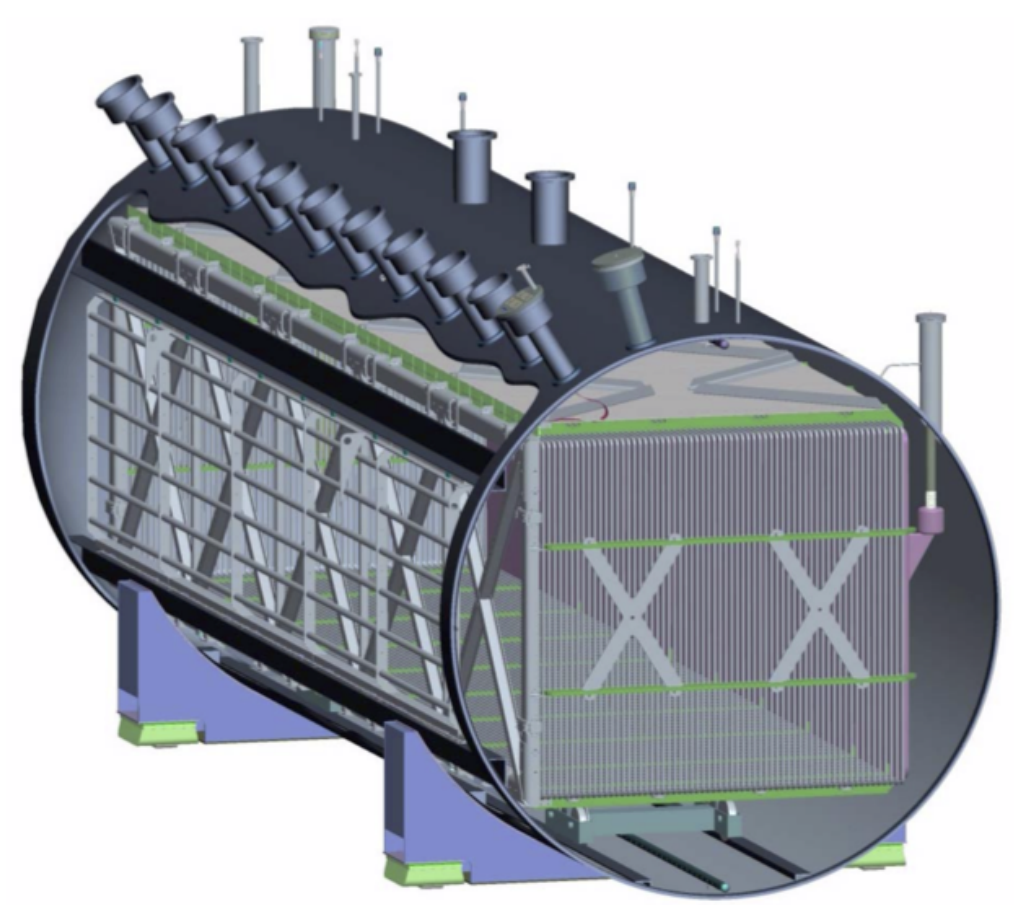

Figure 4.7: 3D rendering of the MicroBooNE cryostat, showing the TPC wire cage and the feedthroughs, which take the signals from the wires and the PMTs (not shown) to the DAQ. In this rendering, the cathode is on the right and the wire planes on the anode side are not shown.

The field cage, made of 64 stainless steel tubes, creates a uniform electric field of $273 \mathrm{~V} / \mathrm{cm}$, stepping down from the $-70 \mathrm{kV}$ at the cathode to almost $0 \mathrm{~V}$ at the anode, using a resistor chain made of eight $10 \mathrm{M} \Omega$ resistors.

The anode consists of three wire readout planes separated by $3 \mathrm{~mm}$ : the drifting electrons induce a bipolar signal in the first two planes $(\mathrm{U}, \mathrm{V})$ at $\pm 60^{\circ}$ inclination, hence their name induction planes, and are then collected by the last plane (Y) at $0^{\circ}$, called collection plane, where they produce a unipolar signal. The induction planes are made by 2400 wires, while the collection plane consists of 3456 vertical wires. The wires are separated by a pitch of $3 \mathrm{~mm}$.

The wire planes are held at different voltages (U plane at $-110 \mathrm{~V}, \mathrm{~V}$ plane at $0 \mathrm{~V}$, and $\mathrm{Y}$ plane at $230 \mathrm{~V}$ ), in order to minimise the amount of charge collected by the induction planes.

The charge deposited in the TPC generates a signal used to create three distinct two-dimensional views (in terms of wire and time) of the event, which can be 
combined to reconstruct a three-dimensional image of the interaction. Figure 4.8 shows a diagram of the MicroBooNE TPC, with the coordinate system used in the experiment.

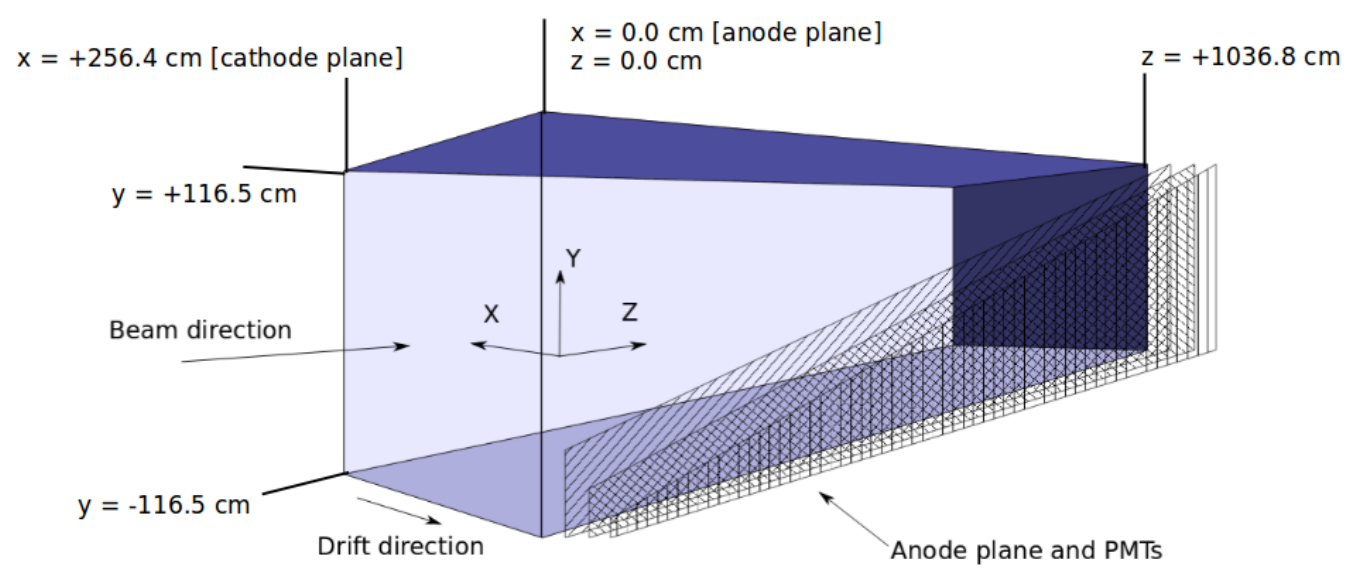

Figure 4.8: Drawing of the MicroBooNE TPC showing the coordinate system and the size of each side. The anode and the cathode are respectively on the right and the left, as seen from the beam.

MicroBooNE started acquiring neutrino data in October 2015. Figure 4.9 shows the event display of a $\nu_{\mu} \mathrm{CC} \pi^{0}$ candidate in the collection plane. The excellent granularity of the detector allows appreciating the electromagnetic showers coming from a $\pi^{0} \rightarrow \gamma \gamma$ decay and also the small $\delta$-rays produced by the cosmic muons. It is also possible to appreciate the challenge of the pattern recognition, with different topologies and overlapping ionisation trails given by the presence of cosmic rays.

\subsubsection{The Light Collection system}

The MicroBooNE light collection system consists of 32 photomultipliers (PMTs) operating in the liquid argon and placed behind the anode plane, which is $86 \%$ transparent to the light. The liquid argon scintillation light has a typical spectrum peaked at $128 \mathrm{~nm}$, which must be shifted to a higher wavelength region, where the PMTs have higher efficiency. MicroBooNE employs tetraphenyl-butadiene (TPB), which absorbs in the UV and emits at $425 \pm 20 \mathrm{~nm}$. The efficiency for transmission through PMT borosilicate glass must also be taken into account 


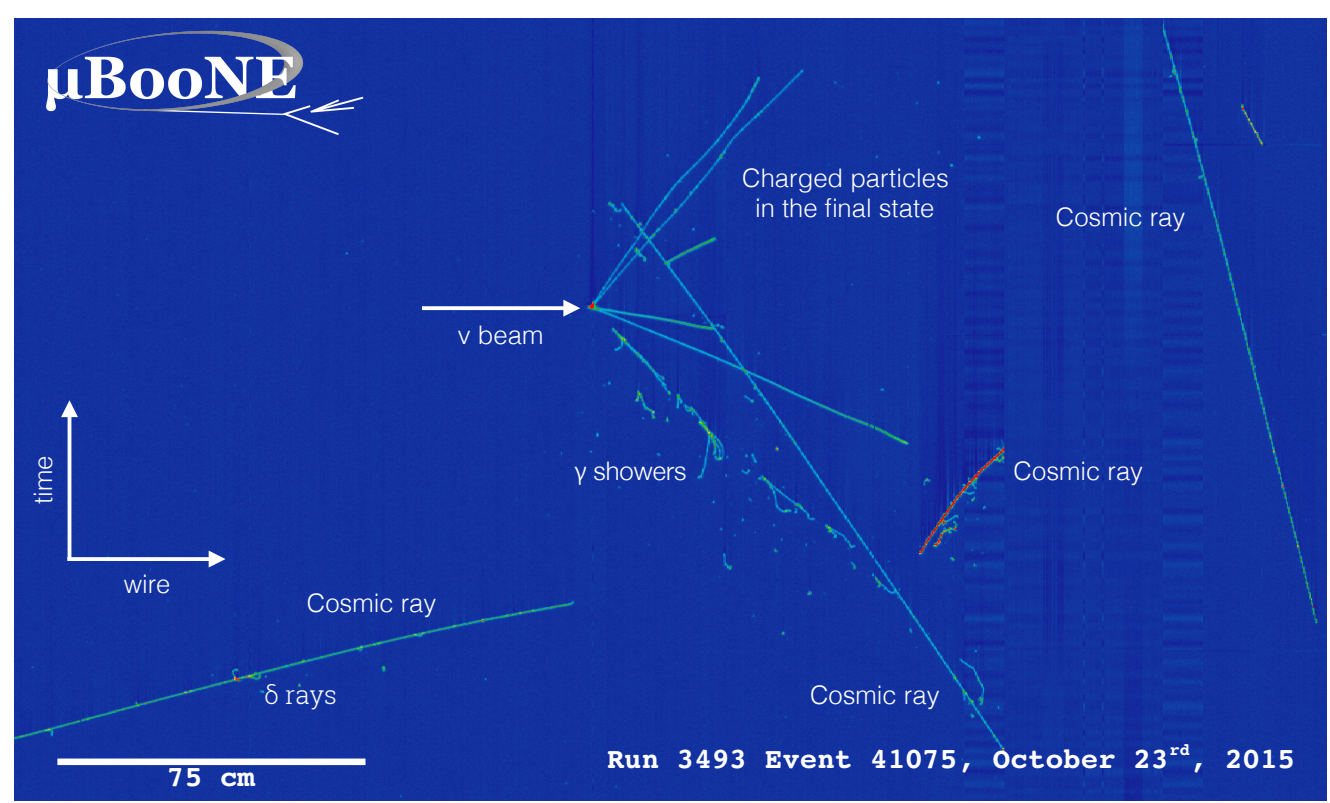

Figure 4.9: Event display of a $\nu_{\mu} \mathrm{CC} \pi^{0}$ candidate in the collection plane. The colour scale corresponds to the amount of charged deposited on the wires.

(around 90\%). Figure 4.10 summarises the light emission and absorption in the MicroBooNE light collection system.

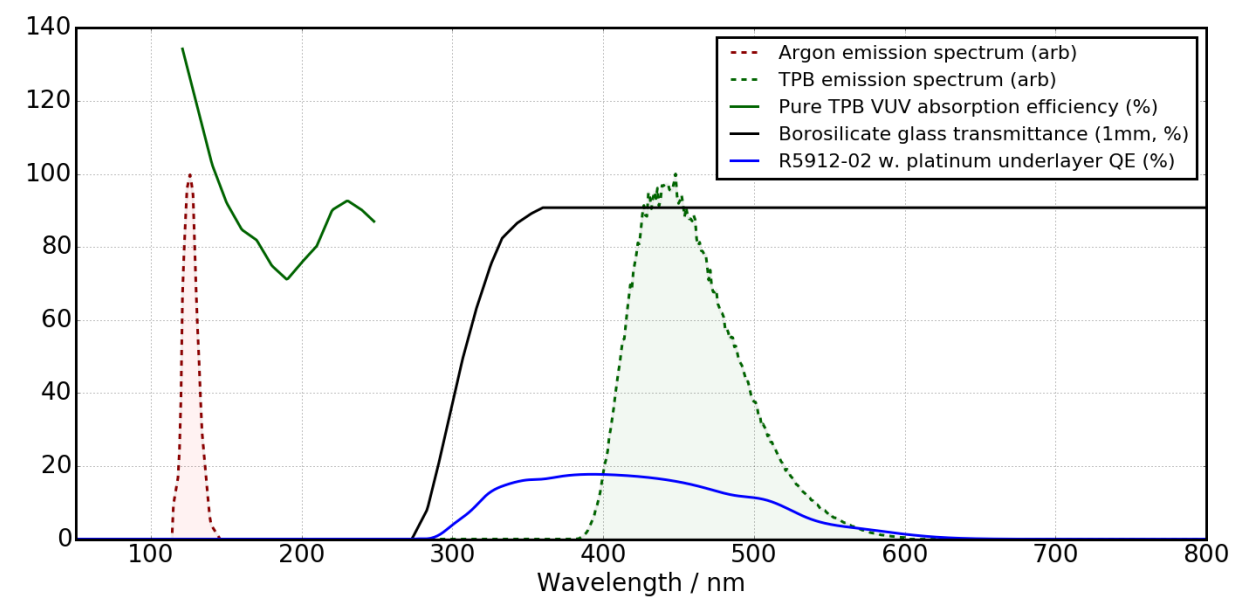

Figure 4.10: Scintillation light emission spectrum (red) and TPB re-emission spectrum (green), with the quantum efficiency of the PMTs employed in MicroBooNE (blue). PMT borosilicate glass transmittance is represented by the solid black line. From 67 .

\subsubsection{Cryogenics and purification}

The liquid argon needs to be kept at a constant temperature and pressure, since they strongly correlate with the drift velocity and, in turn, with the reconstruction 
of the $x$ coordinate.

The temperature is monitored by 12 Resistive Thermal Devices (RTDs) placed in various location of the cryostat, which is covered with insulating foam to prevent large temperature variations.

The purification of the liquid argon is performed by a system of two condensers, two pumps, and two filters. The gaseous argon that leaves the detector enters a condenser kept at boiling temperature by liquid nitrogen coils $(77 \mathrm{~K})$ and it is then pumped into the filters which mainly remove water and $\mathrm{O}_{2}$ molecules. The performances of the purification system can be quantified by measuring the electron lifetime ratio, as shown in Figure 4.4 .

\subsubsection{Electronics and readout}

MicroBooNE readout electronics can be divided into two main parts: one responsible for the digitisation and recording of the TPC wire signals and one responsible for the digitisation and recording of the PMT signals.

TPC readout and electronics The TPC electronics system can be classified into cold electronics, placed inside the cryostat in the liquid argon and responsible for pre-amplification and shaping, and warm electronics, placed outside the cryostat and responsible for digitisation and compression of the signals.

The pre-amplification and shaping of the signals happen in the ASIC CMOS chips on cold motherboards placed near the wires, in order to obtain the lowest possible noise. In particular, the wire noise is reduced by a factor of 2 when going from room temperature to liquid argon boiling temperature [91.

The signals are carried through warm wires to the Analog-to-Digital Converters (ADCs) boards and digitised by a $16 \mathrm{MHz}$ clock. The waveforms are then processed in Front-End Modules (FEMs) and down-sampled to $2 \mathrm{MHz}$. The output consists of time-ordered waveforms of 9600 time-ticks, for a total of $4.8 \mathrm{~ms}$. 
PMTs readout and electronics The signals coming from the 32 PMTs are mainly used in MicroBooNE for the software trigger, as described in Section 4.4.6, and to store information on the light emitted by the liquid argon scintillation.

Signals from the PMTs are first split by a dedicated circuit in a high-gain $(\sim 20 \mathrm{ADC} / \mathrm{PE})$ and a low-gain $(\sim 2 \mathrm{ADC} / \mathrm{PE})$ channel which carry $18 \%$ and $1.8 \%$ of the total signal amplitude, respectively.

Then, they are amplified and shaped with a 60 ns rise time and finally digitised at $64 \mathrm{MHz}$ (15.625 ns time-tick). The waveforms are stored over a window of 1500

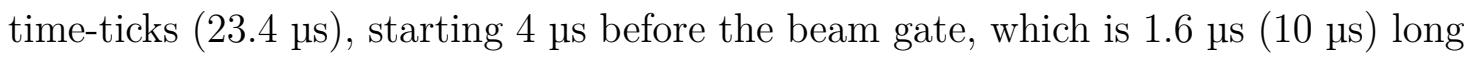
for the BNB (NuMI beam). Digitised waveforms outside this window are stored only if above $130 \mathrm{ADC}$ counts (around 6.5 PE) and for a duration of 40 time-ticks

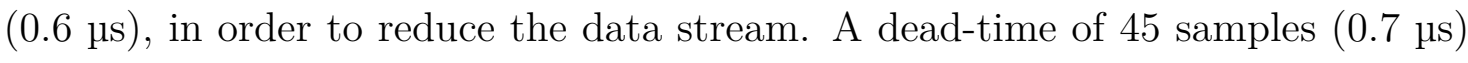
follows each recorded out-of-beam-gate digitised waveform.

\subsubsection{The MuCS and the Cosmic-Ray Tagger}

Being located almost on surface, the MicroBooNE detector is constantly subject to a constant $\sim 5.5 \mathrm{kHz}$ cosmic-ray rate 92 , which corresponds to around 13 cosmic rays for a drift-time window of $2.3 \mathrm{~ms}$.

In order to study the challenges of cosmic-ray reconstruction, the MicroBooNE detector was also equipped with a small external muon counter stack (MuCS), installed at the start of operations in 2015.

This sub-detector was used to measure for the first time the cosmic-ray reconstruction efficiency in a LArTPC. This measurement is described in detail in the publication reported in Appendix A, whose corresponding author is the author of this thesis. Triggering on the signal coming from this small muon counter stack allowed to measure the fraction of cosmic-ray tracks effectively reconstructed in the LArTPC. The measured efficiency is $97.1 \%$, in agreement with the Monte Carlo simulation.

This study was also used to demonstrate the tagging capabilities of a larger Cosmic-Ray Tagger (CRT), which surrounds the cryostat on four sides and represents the first cosmic-ray tagging system integrated with a LArTPC 93 . Figure 4.11 
shows a simulation of the cosmic rays which hit the LArTPC and are tagged by the MuCS and the CRT.

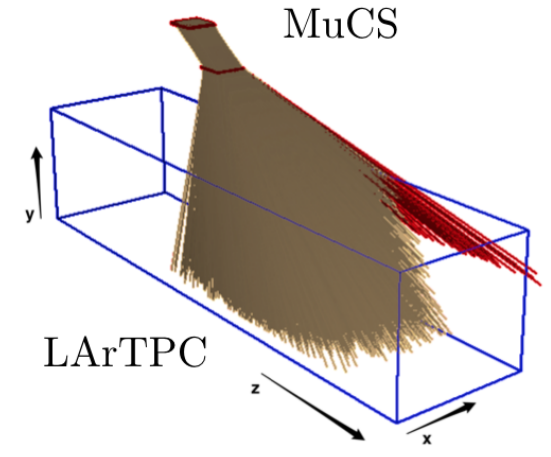

(a) Cosmic rays hitting the MuCS. Red lines correspond to cosmic rays hitting the MuCS but missing the LArTPC.

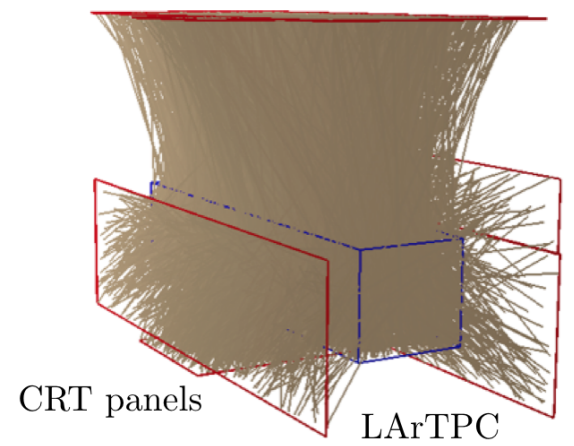

(b) Cosmic rays hitting at least of the one of the CRT panels and the LArTPC.

Figure 4.11: 3D drawing of simulated cosmic rays (brown lines) hitting the LArTPC and tagged by the MuCS (left) and the CRT (right).

This sub-system was installed at Fermilab in 2017 and was not present when the data used in this thesis was acquired.

The CRT panels are made of plastic scintillation modules, which provide time and position information for charged particles crossing the panels and hitting the TPC. Cosmic-ray rejection in the LArTPC can be improved both by spatially tagging crossing cosmic rays and by vetoing events with a signal in the CRT during the beam-gate window.

\subsubsection{Trigger system}

MicroBooNE employs different triggers in order to minimise the amount of stored data, while keeping a very high neutrino efficiency.

A hardware trigger is fired for each beam spill in the Booster and in the NuMI neutrino beams. When received, a 23.4 ss window is opened in the PMT readout and a $4.8 \mathrm{~ms}$ window is opened in the TPC readout. These two triggers (one for the BNB and one for NuMI) have the highest priority, and in case the two beam-gate windows overlap, the precedence is given to the BNB. The beam trigger efficiency is $99.8 \%$. 
Each event in MicroBooNE requires around $30 \mathrm{MB}$ of storage which, for a $5 \mathrm{~Hz}$ beam-trigger rate, would correspond to $13 \mathrm{~TB} /$ day. However, most beam spills do not produce an effective neutrino interaction in the detector. In order to minimise the number of events containing only cosmic rays, thus reducing the amount of data stored, the beam trigger is required to be in coincidence with a PMT trigger, implemented at software level. In this way, it is possible to achieve a higher level of sophistication and makes the Monte Carlo simulation of the trigger easier.

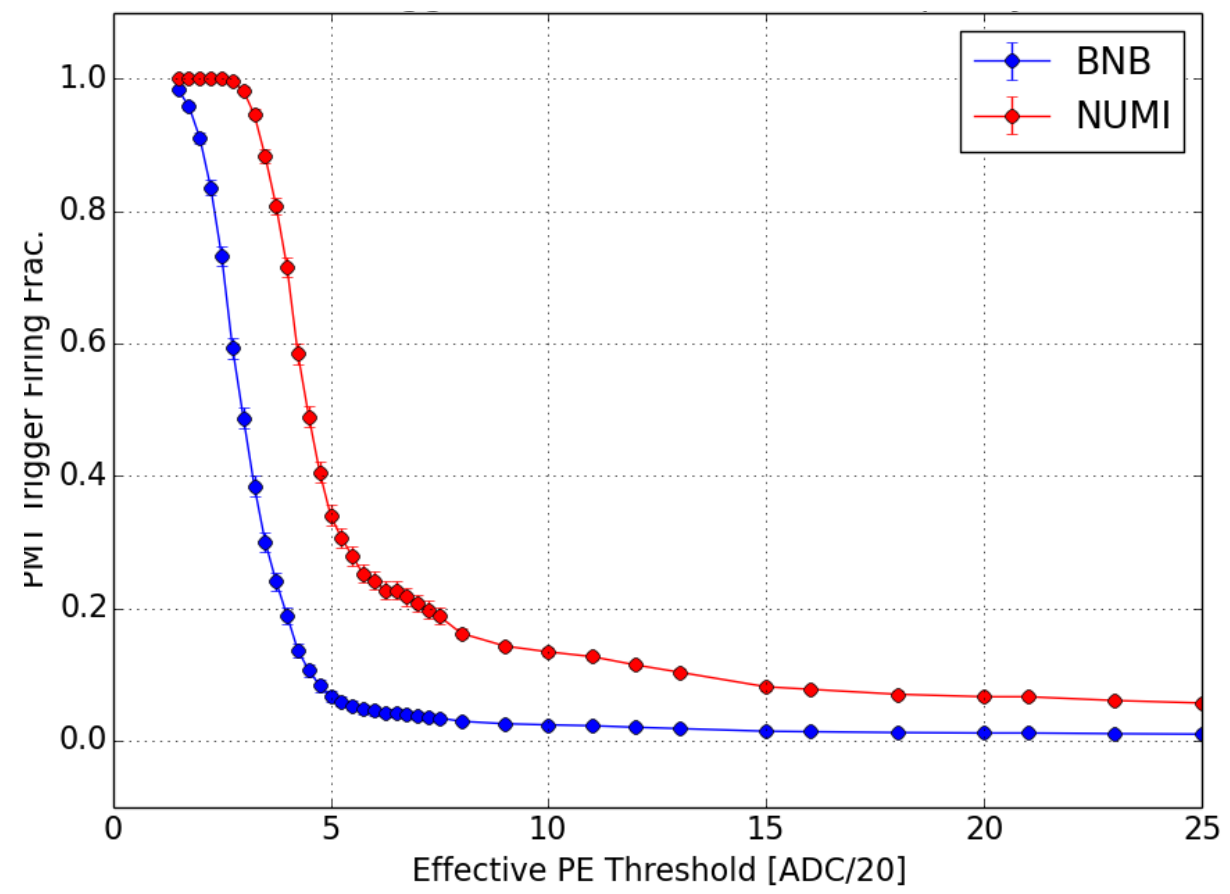

Figure 4.12: Software trigger efficiency as a function of the PE threshold, both for BNB (blue) and NuMI (red) beam-gate windows.

The software trigger requires 6.5 effective $\mathrm{PE}$ (photoelectrons) in the light collection system during the beam gate window, rejecting around $97 \%$ of the beam spills. Figure 4.12 shows the software trigger efficiency as a function of the PE threshold, both for BNB and NuMI beam-gate windows. The NuMI beam trigger has a higher efficiency compared with the BNB beam trigger because the neutrinos are on average more energetic.

Cosmic rays can still produce background events if they cross the TPC during the beam gate window, and produce enough scintillation light. In order to precisely assess this background, a trigger, called EXT, is fired at a $0.1 \mathrm{~Hz}$ rate orthogonally 
to the beam-gate windows, requiring $6.5 \mathrm{PE}$ in a $1.6 \mathrm{\mu s}$ time window (same as the BNB beam trigger). Events selected by this beam-off trigger will then contain only cosmic rays.

Figure 4.13 shows the time-distribution of optical flashes recorded from events triggered during the BNB beam-gate window. An optical flash is a group of light pulses recorded by the PMTs within a 100 ns time difference. The excess of events corresponds to the $1.6 \mu$ s beam spill width.

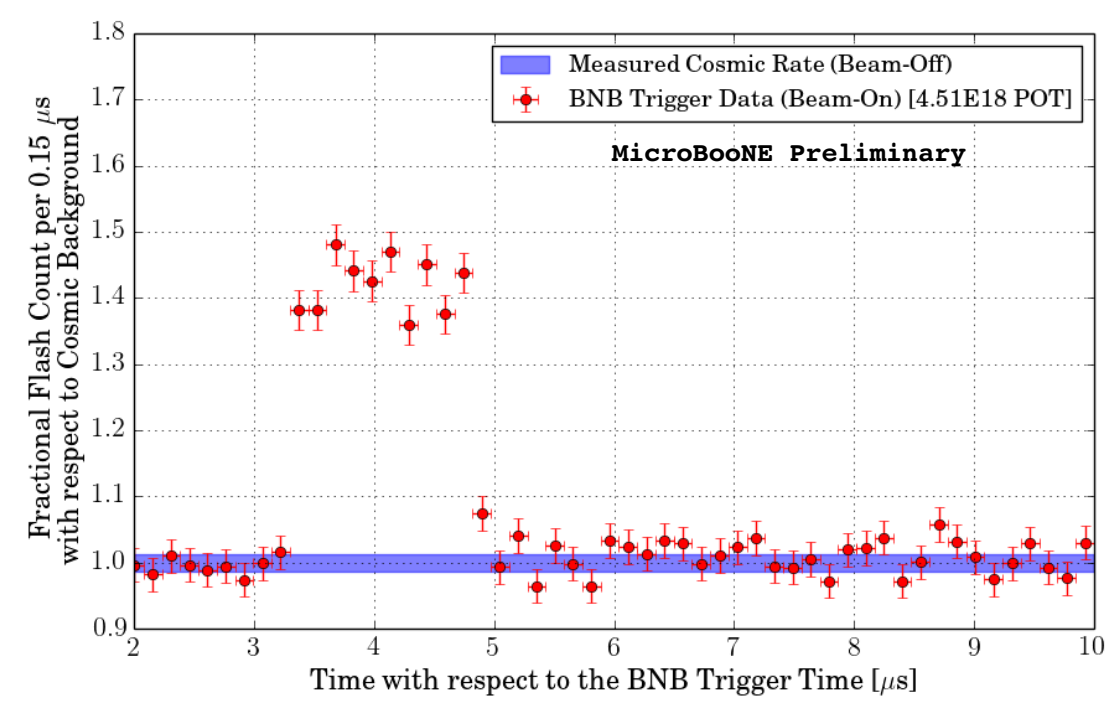

Figure 4.13: Distribution of optical flash times with respect to the trigger time for BNB triggered events, shown as a ratio to the expected cosmic rate from beam-off data, collected with the EXT trigger.

The DAQ (data acquisition) system takes as input the triggered TPC and PMT readouts and translates the raw data format into ROOT files, one for each trigger. As of January 2019, MicroBooNE has collected $1.2 \times 10^{21}$ POT in BNB neutrino mode, which roughly corresponds to the amount of POT collected by MiniBooNE (which collected also $1.1 \times 10^{21}$ POT in antineutrino mode and $1.9 \times 10^{20} \mathrm{POT}$ in beam dump mode). This document will focus on $4.34 \times 10^{19}$ POT collected with the BNB trigger between February 23 and May 22, 2016, which corresponds to MicroBooNE open data sample. 


\section{Event reconstruction}

\section{Contents}

$5.1 \quad$ Signal processing $\ldots \ldots \ldots \ldots \ldots \ldots$

5.1 .1 Noise removal . . . . . . . . . . . . . . . . 58

$5.1 .2 \quad$ Signal deconvolution . . . . . . . . . . . . . . . . 59

5.1 .3 Hit reconstruction . . . . . . . . . . . . . . . 61

5.2 The Pandora multi-algorithm pattern recognition . . . 61

In this section we will describe the process which transforms the raw digitised waveforms collected by the DAQ into high-level objects used for physics analysis.

\subsection{Signal processing}

\subsubsection{Noise removal}

The ionisation electrons moving towards the anode induce a bipolar signal on the induction planes and produce a unipolar signal on the collection plane, as described in Section 4.4.1. These signals need to be extracted and processed after the noise sources, described below, have been removed or mitigated.

The ASIC chips placed in the liquid argon produce an inherent irreducible noise, caused by thermal fluctuations and charge trapping and de-trapping in the input transistor 94 . 
The MicroBooNE readout electronics is also affected by excess from three main sources, whose identification and mitigation is thoroughly described in 94 . Here we provide a brief overview for informative reasons.

Low-voltage regulators noise. The ASIC chips are fed by low-voltage regulators which introduce a noise across all channels at around $30 \mathrm{kHz}$ in the frequency spectrum. This component represents the most significant excess noise source. To mitigate it, a correction waveform is constructed on a per sample basis and subtracted from each channel.

HV power supply noise. This noise is induced by the cathode high-voltage power supply around $36 \mathrm{kHz}$ and $108 \mathrm{kHz}$. An offline filter directly removes this harmonic noise in the frequency domain.

$900 \mathrm{kHz}$ burst noise. The source of this noise is still unknown. It is positiondependent and has a burst nature. The main hypothesis points towards the PMT high-voltage supply. This noise is attenuated by the anti-alias filter and at the moment is not actively mitigated.

The entire noise-reduction filtering chain allows to increase the peak signal-to-noise ratio by a factor of 2 in the collection plane (from 19.5 to 37.9 ) and by a factor of 3 in the induction planes (from 6.6 to 22.3 in the $\mathrm{U}$ induction plane and from 5.7 to 16.2 in the $\mathrm{V}$ induction plane). As an example, Figure 5.1 shows an event display of the $\mathrm{V}$ induction plane before and after the noise removal: a clear neutrino interaction emerges with the improvement in the signal-to-noise ratio.

\subsubsection{Signal deconvolution}

After the noise-filtering stage, a first reconstruction step searches for regions-ofinterest (ROI) in the wire signals. Thus, the signal deconvolution step aims to disentangle the detector electronic response from the original profile of the charge 


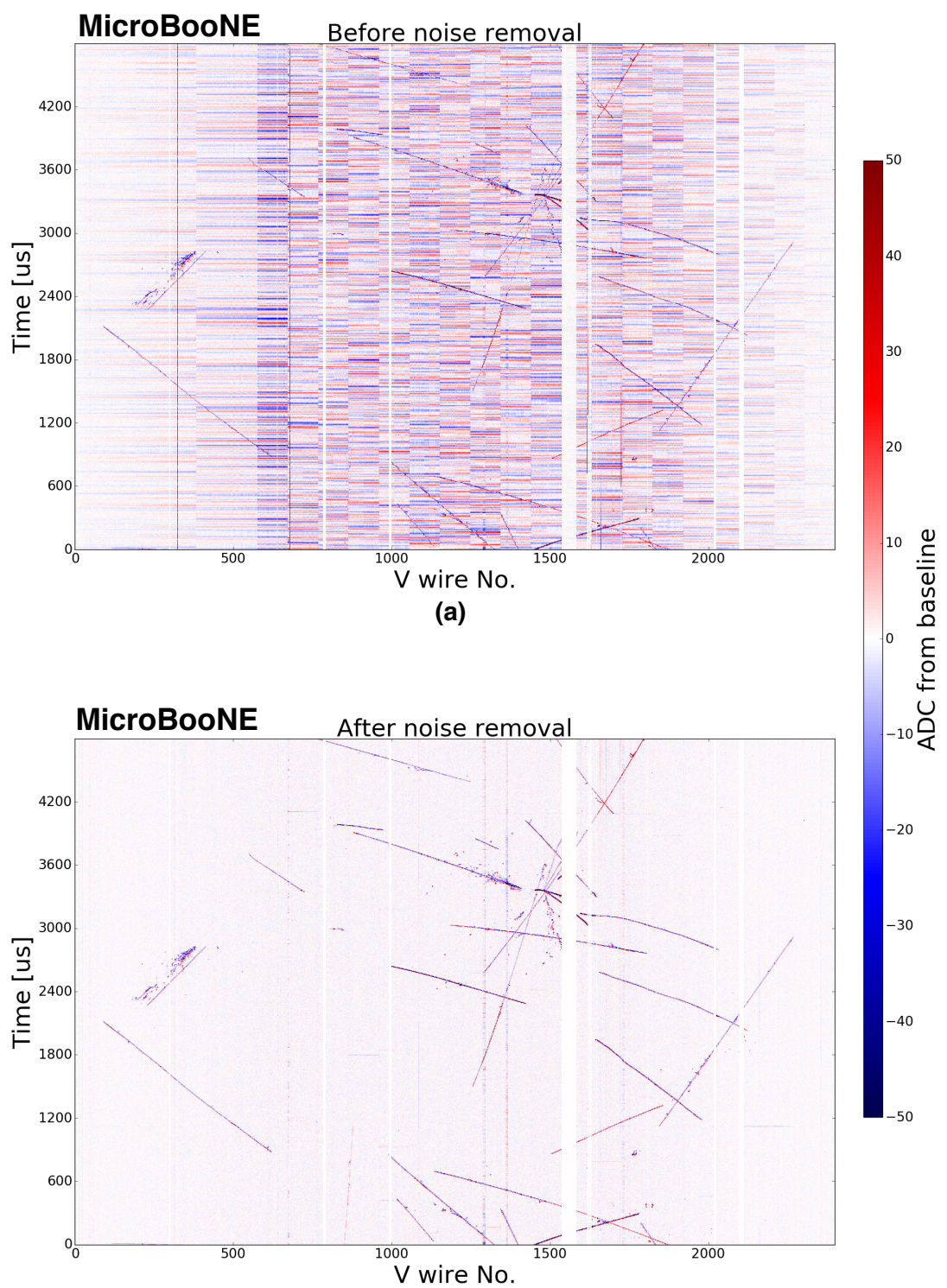

(b)

Figure 5.1: Data event display of the V induction plane showing the raw signal before (a) and after (b) offline noise filtering. The horizontal axis corresponds to the wire coordinate and the vertical axis correspond to the drift time. The colour scale represents the ADC counts from the baseline in arbitrary units. From 94 . 
collected or induced on the wires. This process is usually performed with a Fourier transform of the measured signal, which yields:

$$
S(\omega)=\frac{M(\omega)}{R(\omega)}
$$

where $S(\omega)$ is the original signal, $M(\omega)$ is the measured signal, and $R(\omega)$ is the response function, all in the frequency domain. However, the response function typically decreases at high frequencies, leading to increased noise in this region of the frequency domain. This issue is solved by applying a filter function $F(\omega)$, whose details are thoroughly described in $|95|$.

\subsubsection{Hit reconstruction}

The final stage of the signal processing tries to perform one or more Gaussian fits to the deconvolved signals. Each Gaussian fit corresponds to a reconstructed hit. The time of the reconstructed hit is the mean of the Gaussian and its RMS corresponds to the Gaussian width. The integral of the fitted function gives the charge associated to the hit. Figure 5.2 shows a data event display of the collection plane, with two peaks in the ROI, produced by the ionisation trails of two cosmic rays. These reconstructed hits are finally passed to the pattern recognition framework, which aims to reconstruct $2 \mathrm{D}$ and $3 \mathrm{D}$ clusters.

\subsection{The Pandora multi-algorithm pattern recog- nition}

The results shown in this thesis were produced using the Pandora Software Development Kit for pattern recognition. This general-purpose framework was specifically developed to identify energy deposits in high-granularity detectors, such as LArTPCs 96 .

Several algorithms are applied in sequence to the input information (in our case the reconstructed hits), which gradually build up a complete picture of the event.

As a first step, input hits are separated into three different lists, one for each plane. Then, for each list, an algorithm groups together continuous and unambiguous lines 

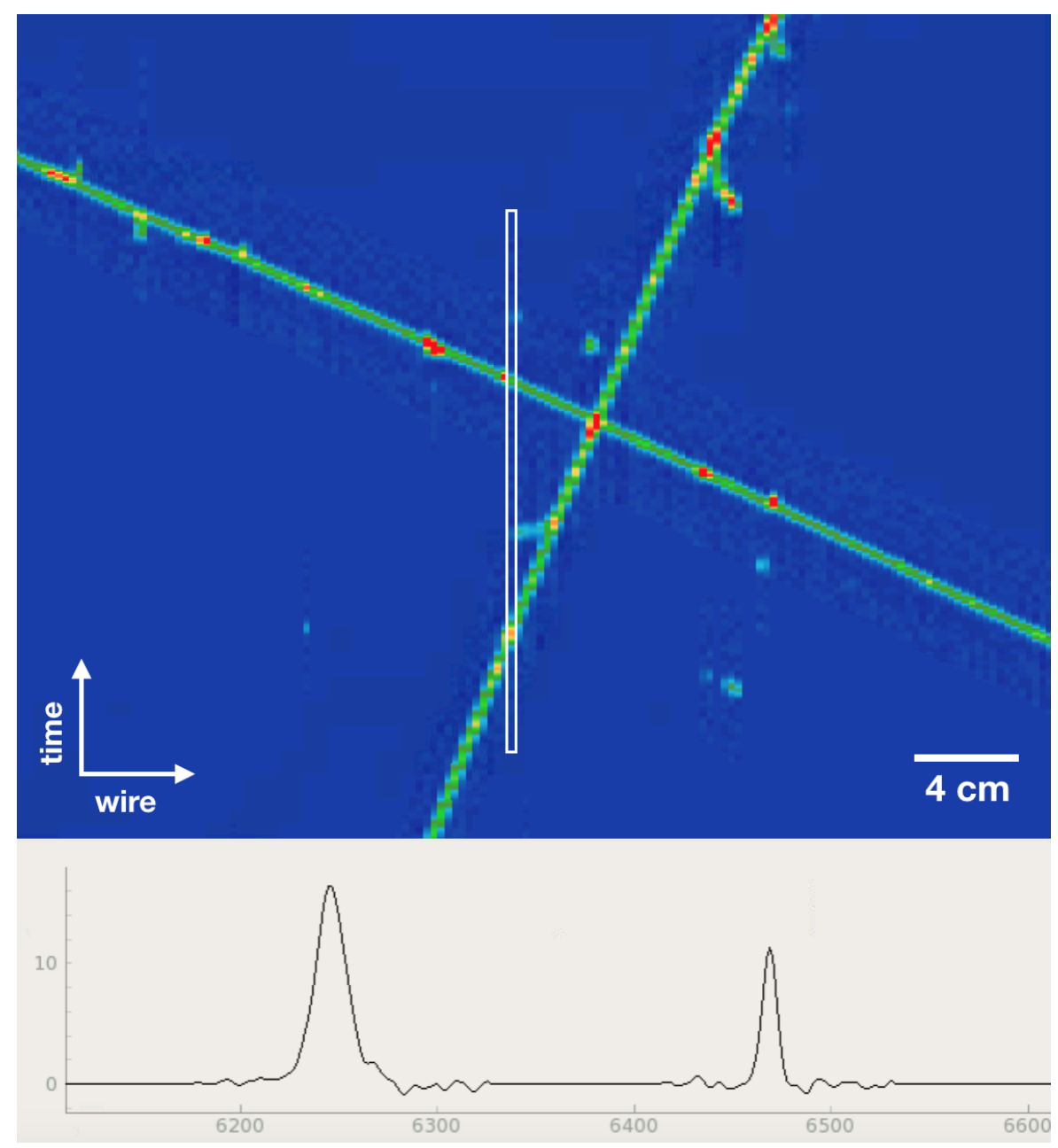

Figure 5.2: Data event display with the waveform collected in a wire of the collection plane. The white box corresponds to the waveform in the bottom part. The units of the $\mathrm{x}$-axis are in $500 \mathrm{~ns}$ time-ticks.

of hits. These two-dimensional clusters are then examined by a series of topological algorithms. Given the presence of unresponsive wires in the MicroBooNE detector, a continuous ionisation trail can be reconstructed as two or more separate clusters. The topological algorithms try to stitch different clusters together, if e.g. they point towards the same direction or if they are in close proximity.

Once the topological algorithms deem the clustering in each plane as complete, the $3 \mathrm{D}$ reconstruction algorithms take as input the clusters from the three planes and aim to reconstruct the three-dimensional objects, which in Pandora are called PFParticles (Particle Flow Particles). Subsequently, a Support Vector Machine trained on Monte Carlo uses the topological and geometrical properties of the object 


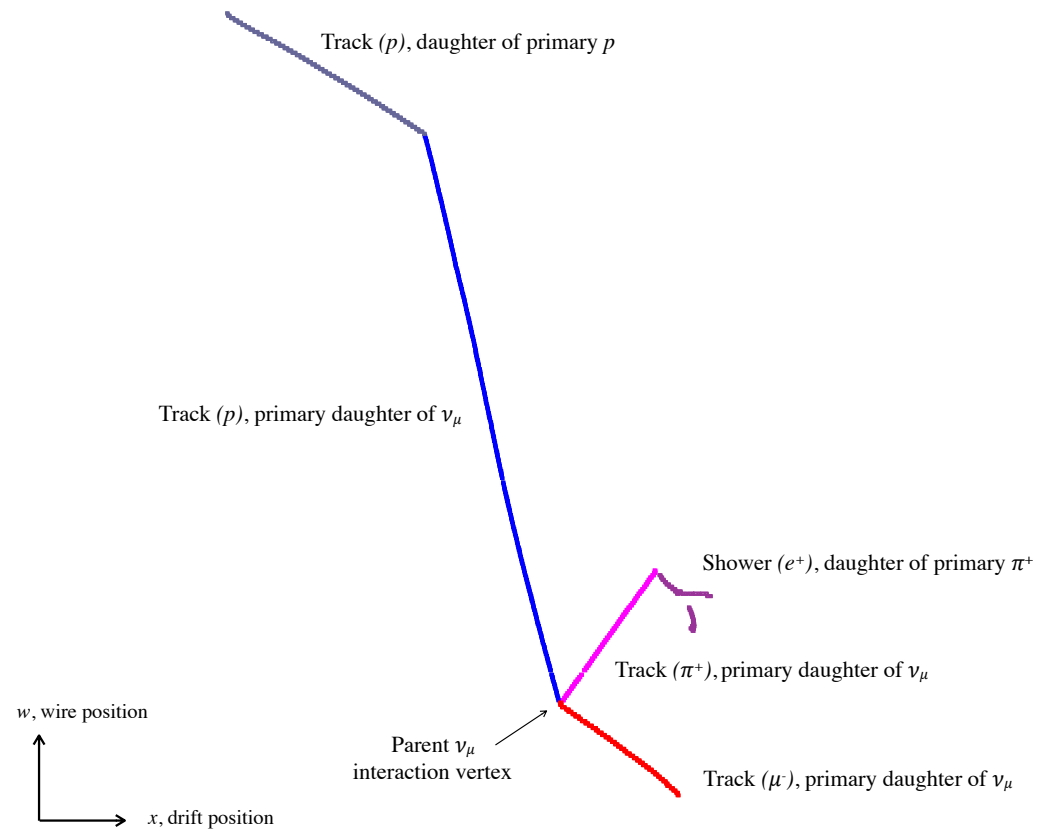

Figure 5.3: Pandora pattern recognition output of a simulated CC $\nu_{\mu}$ event with a muon (red), a proton (blue) and a charged pion (pink) in the final state. Each particle is reconstructed as a separate cluster and the positron coming from the $\pi^{+} \rightarrow \mu^{+} \rightarrow e^{+}$ decay chain is classified as a daughter of the pion. From 97 .

to classify it as track or as a shower. An important feature of the Pandora pattern recognition is its ability to create a hierarchy of reconstructed PFParticles. As an example, the reconstructed shower corresponding to a Michel electron will be considered as the daughter of the reconstructed track corresponding to the stopping muon. This capability is particularly important for the reconstruction of complex neutrino interactions, as illustrated in Figure 5.3 .

Pandora can also run in two modes: one optimised for the reconstruction of cosmic rays and delta rays, the cosmic mode, and one optimised for the reconstruction of neutrino interactions, the neutrino mode. These two modes are used sequentially in our analysis in order to suppress the cosmogenic background, as described in Section 6.2.3. 


\section{Selection of electron neutrinos in the MicroBooNE experiment}

\section{Contents}

6.1 Signal definition $\ldots \ldots \ldots \ldots \ldots$

6.2 Analysis Methodology . . . . . . . . . . 67

$6.2 .1 \quad$ Data and Monte Carlo samples . . . . . . . . . . . . 68

6.2 .2 Overview of the analysis . . . . . . . . . . . . . . . . . . . . 69

6.2 .3 Cosmic-ray rejection . . . . . . . . . . . . . . . 71

6.2 .4 Optical selection . . . . . . . . . . . . . . . . 73

6.2 .5 Topological pre-selection . . . . . . . . . . . . . 77

$6.2 .6 \quad$ Minimum reconstruction quality requirements. . . . . . . 78

6.2 .7 Selection efficiency and purity . . . . . . . . . . . . . . 79

6.2 .8 Selection performances in BNB events . . . . . . . . . 84

6.3 Calorimetry ......................... 88

6.3 .1 Scope of the energy reconstruction . . . . . . . . . 88

6.3 .2 Electron energy reconstruction and calibration . . . . . 90

$\begin{array}{lll}6.3 .3 & \text { Single proton energy reconstruction and calibration. . . } 94\end{array}$

6.3 .4 Deposited Energy Reconstruction . . . . . . . . . . . 96

$6.3 .5 \quad$ Deposited energy binning $\ldots \ldots \ldots$. . . . . . . . 98

\begin{tabular}{lll}
\hline 6.3 .6 & Measurement of the electromagnetic shower energy loss & 99
\end{tabular}

6.3 .7 Particle identification of reconstructed tracks . . . . . . 103

6.4 Background Rejection . . . . . . . . . . . 106

6.4 .1 Rectangular cuts . . . . . . . . . . . . . . . . 106

6.4 .2 Boosted Decision Trees $\ldots \ldots$. . . . . . . . . . . . . 120

6.4 .3 Interaction types . . . . . . . . . . . . . . . . . 128

6.5 Validation . . . . . . . . . . . . . . . . 128

$6.5 .1 \quad$ Electromagnetic shower energy loss . . . . . . . . . . 128

6.5 .2 Side-bands checks $\ldots \ldots \ldots$. . . . . . . . . . . . 129 
6.5 .3 NuMI beam event studies . . . . . . . . . . . . . 131

One of the main physics goals of the MicroBooNE experiment is to search for and clarify the low-energy excess of electron-like events observed by the MiniBooNE experiment 48 .

However, the MiniBooNE experiment employs a Cherenkov detector, which does not have the ability to distinguish between single electrons and single photons in the final state. This technology limitation makes it very challenging to identify a physics model that could definitely explain the excess.

The MicroBooNE detector, being a LArTPC, provides detailed calorimetry, which makes it possible to measure the $d E / d x$ of ionisation tracks and electromagnetic showers [67, and excellent granularity, which allows to measure the gap between the neutrino interaction vertex and the start of the electromagnetic shower. These two methods provide powerful electron/photon separation.

In this chapter we will describe a fully-automated electron neutrino selection using the Pandora multi-algorithm pattern recognition. This is the first fullyautomated electron neutrino search in a LArTPC. The selection will be validated with two orthogonal side-bands, one enriched with neutral-current interactions (both $\nu_{\mu}$ and $\nu_{e}$ ) and one enriched with charged-current $\nu_{\mu}$ interactions. It will also be applied to an independent sample of neutrino events acquired with the NuMI beam. The systematic uncertainties will evaluated in Chapter 7 and the current sensitivity to the MiniBooNE low-energy excess in the electron hypothesis will be estimated in Chapter 8

\subsection{Signal definition}

The MiniBooNE experiment showed an excess of CCQE-like events in the 200$475 \mathrm{MeV}$ neutrino energy range [48, therefore this analysis will focus on a similar topology.

Our selection aims to have a sample with one electron, no other leptons or photons, at least one proton, and no other charged hadrons or mesons in the final 
state. All particles in the final state are required to be above detection thresholds, evaluated in Section 6.2.7. These events are called $\nu_{e} \mathrm{CC} 0 \pi-\mathrm{Np}($ where N > 0) 47.

In MicroBooNE, a $\nu_{e} \mathrm{CC} 0 \pi-\mathrm{Np}$ interaction corresponds to an electromagnetic shower, produced by the electron, and one or more ionisation tracks, produced by the protons.

The channel closest to the CCQE-like events selected by MiniBooNE is $\mathrm{CC} 0 \pi$ (so including events with 0 protons), since the Cherenkov threshold for protons in mineral oil is $350 \mathrm{MeV}$ (kinetic energy) 98. However, the choice of the $\mathrm{CC} 0 \pi-\mathrm{Np}$ channel presents several advantages, described below.

Easier pattern recognition. The presence of a proton in the final state makes vertexing and pattern recognition easier, since there will be typically a kink between the ionisation track and the electromagnetic shower.

Improved energy resolution. As shown in Section 6.3, the length of the ionisation tracks can be used to estimate the energy deposited by the protons. This method provides a higher energy resolution than the one obtained by collecting the deposited charge.

Improved background rejection. Requiring an ionisation track allows to reject $\mathrm{NC} \pi^{0}$ and $\mathrm{CC} \pi^{0}$ interactions with no protons in the final state and where one of the $\pi^{0} \rightarrow \gamma \gamma$ is not reconstructed or escapes the detector. These events represent a large background of our analysis. Also, the presence of the proton allows to reject events with a photon converting far from the interaction vertex, as it makes possible to measure the gap between the shower and the interaction vertex.

As an example, Figure 6.1 shows a simulated $\nu_{e} \mathrm{CC} 0 \pi-\mathrm{Np}$ event display on the collection plane with an electron and two protons in the final state, with the corresponding reconstructed shower and reconstructed tracks. In this case, the pattern recognition is able to correctly identify the electromagnetic shower and both proton tracks. 


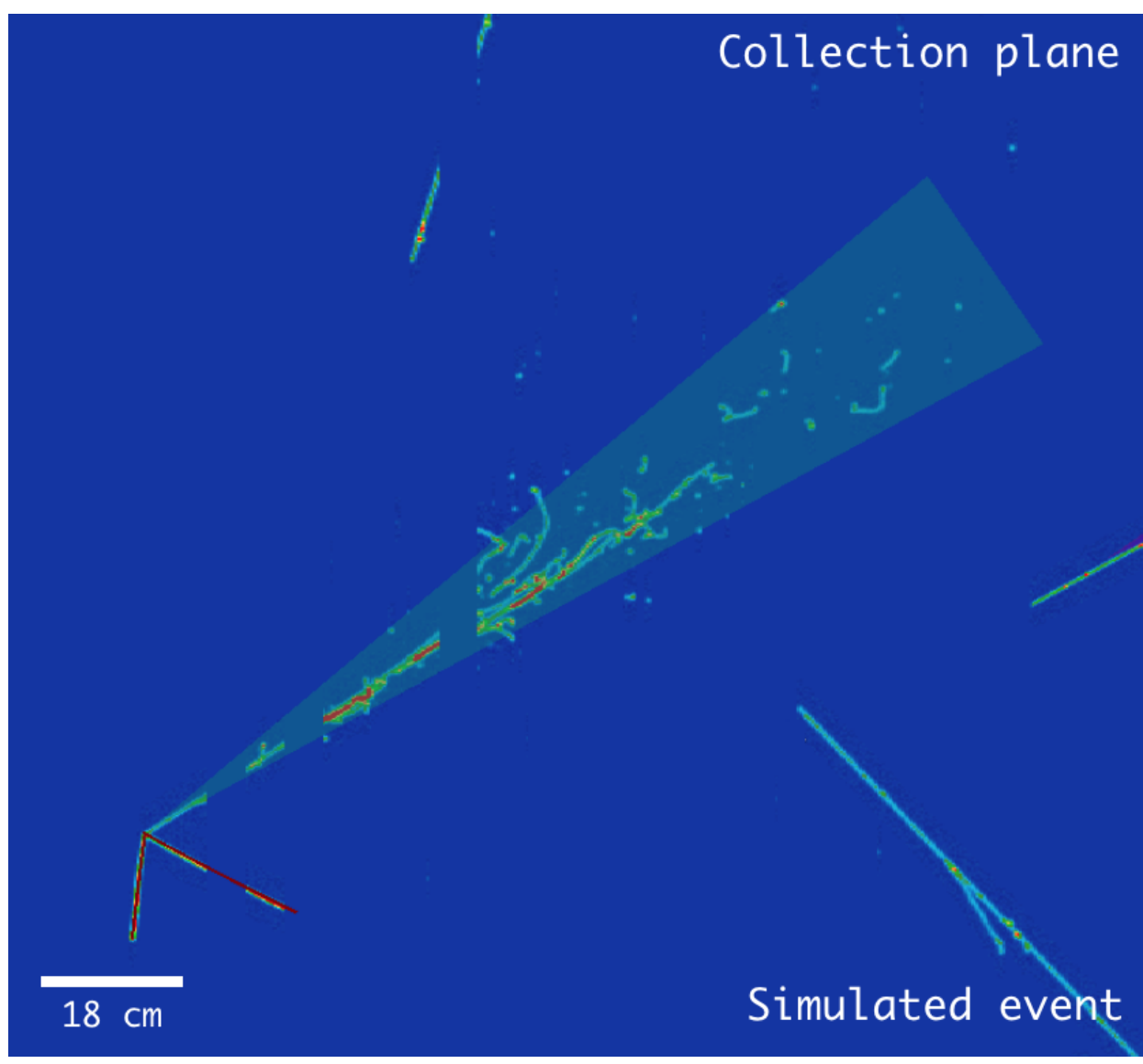

Figure 6.1: Monte Carlo $\nu_{e} \mathrm{CC} 0 \pi-\mathrm{Np}$ event display of the collection plane with an electron and two protons in the final state. The reconstructed shower-like object is represented by the green cone. The reconstructed track-like objects are represented by the red lines. The ionisation trails without an associated reconstructed track are cosmic rays correctly tagged by the cosmic-removal algorithms, described in Section 6.2.3. The colour scale is proportional to the amount of charge collected by the wires. The vertical gaps are caused by the presence of unresponsive wires in the detector, which are turned off in the simulation.

\subsection{Analysis Methodology}

The goal of the event selection is to obtain a sample enriched with $\nu_{e} \mathrm{CC} 0 \pi-\mathrm{Np}$ interactions. The results of the event selection are described in detail in Section 6.2.8. In order to increase the purity, two parallel background rejection strategies have been developed: one with rectangular cuts on kinematic and calorimetric variables, described in Section 6.4.1, and one with Boosted Decision Trees, described in Section 6.4.2. The measurement of the systematic uncertainties affecting the variables used in the background rejection is described in Chapter 7. The full 
covariance matrix has then be used to measure the sensitivity to the MiniBooNE low-energy excess signal in the electron hypothesis, in Chapter 8 .

\subsubsection{Data and Monte Carlo samples}

In this document, we will analyse a sub-sample of the data collected by the detector between February 23 and May 22, 2016. This sub-sample corresponds to an exposure of the MicroBooNE detector of $4.34 \times 10^{19}$ POT. This represents MicroBooNE unblinded sample for reconstruction, event selection development, and performance measurement. The sample is statistically too small to be sensitive to a MiniBooNElike low-energy excess signal.

The data used for this analysis correspond to two separate samples: the data beam-on, obtained with the BNB beam trigger, and the data beam-off, obtained with the EXT trigger. These two triggers criteria were described in Section 4.4.6.

In order to increase the simulated statistics of our $\nu_{e} \mathrm{CC} 0 \pi-\mathrm{Np}$ events, three different Monte Carlo samples were produced:

$\nu_{e} \mathbf{C C 0} \pi-\mathrm{Np}+$ cosmic sample. Each event has a simulated $\nu_{e}$ interaction in the MicroBooNE cryostat and simulated cosmic rays hitting the detector in the same readout window. The interaction is defined as $\nu_{e} \mathrm{CC} 0 \pi-\mathrm{Np}$ if it has one electron, at least one proton, no photons, and no mesons (pions, kaons) above detection threshold. The start and end points of the protons and the start point of the electron are required to be contained within the fiducial volume, as defined in Section 6.2.6. This sample will be used to assess the reconstruction efficiencies of the analysis.

BNB + cosmic sample. Each event has a simulated neutrino interaction inside the MicroBooNE cryostat, where the neutrino flavours are weighted according to the BNB neutrino flux composition (see Section 4.3), and simulated cosmic rays hitting the detector in the same readout window. This sample will be used to understand backgrounds coming from other neutrino interactions. 
Dirt sample. Each event has a simulated neutrino interaction outside the MicroBooNE cryostat, where the neutrino flavours are weighted according to the BNB neutrino flux composition, and simulated cosmic rays hitting the detector in the same readout window. This sample will be used to understand background events coming from interactions outside the fiducial volume.

Neutrino events have been generated using the GENIE Neutrino Monte Carlo generator version 2.8.6 99] and cosmic rays have been generated using the CORSIKA Monte Carlo generator version 7.4003 [100. Simulated secondary particle propagation employs GEANT version 4.9.6 89, and detector response simulation and reconstruction is performed with the LArSoft framework version 6.26.01.10 101.

\subsubsection{Overview of the analysis}

The reconstruction and selection chain to identify $\nu_{e} \mathrm{CC} 0 \pi-\mathrm{Np}$ electron neutrino candidate events for this analysis is divided into several stages, illustrated in Figure 6.2 and described below.

\begin{tabular}{|c|c|c|c|}
\hline $\begin{array}{l}\text { Cosmic-ray } \\
\text { removal }\end{array}$ & selection & Calorimetry & $\begin{array}{l}\text { Background } \\
\text { rejection }\end{array}$ \\
\hline $\begin{array}{l}\text { Pandora cosmic } \\
\text { pattern } \\
\text { recognition } \\
\text { Dedicated cosmic- } \\
\text { ray removal }\end{array}$ & $\begin{array}{l}\text { - } \text { Topology and light } \\
\text { requirements } \\
\text { - } \quad \text { CC } v_{\mu} \text { rejection }\end{array}$ & $\begin{array}{ll}\text { - } & \text { Shower dE/dx } \\
\text { - } & \text { Track dE/dx } \\
\text { - } & \text { Hit-based } \\
& \text { shower energy } \\
\text { - } & \text { Length-based } \\
& \text { proton energy }\end{array}$ & $\begin{array}{ll}\text { - } & \text { Rectangular cuts } \\
\text { - } & \text { BDTs }\end{array}$ \\
\hline
\end{tabular}

Figure 6.2: Schematics of the $\nu_{e} \mathrm{CC} 0 \pi$-Np event selection stages, from the cosmic-ray removal to the rejection of the neutrino and cosmogenic backgrounds.

Cosmic-ray removal. In order to suppress the cosmogenic background, the first step is to run the Pandora algorithms optimised to reconstruct and remove cosmic rays 97. After this step, hits associated with objects deemed as cosmicinduced by several tagging algorithms, external to Pandora and described in Section 6.2.3, are removed from the event. The remaining hit collection provides the input to the Pandora neutrino reconstruction path, which outputs a list of candidate neutrinos. 
Optical selection. A minimum amount of photoelectrons in the optical detection system is required to be coincident with the BNB beam window and at least one of the neutrino candidates provided by the Pandora framework must be compatible with the flash observed in the optical detection system. These requirements are described in detail in Section 6.2.4.

Electron neutrino topological pre-selection. One of the neutrino candidates must be compatible with the topology of a $\nu_{e} \mathrm{CC} 0 \pi$-Np interaction. Rather than accepting strictly $N$ tracks and one shower, at least one track and at least one shower or at least two showers sharing a common vertex are accepted, due to the presence of split showers and split tracks. Multiple showers without reconstructed tracks are accepted due to a current track/shower identification inefficiency, addressed in Section 6.2.7.

CC $\nu_{\mu}$ neutrino candidates removal. Events tagged as $\mathrm{CC} \nu_{\mu}$ neutrino candidates are rejected by an independent $\mathrm{CC} \nu_{\mu}$ selection module, described in 102 .

Calorimetric variables reconstruction. The energy of the electron showers is measured with a calorimetric procedure, converting the collected charge into deposited energy, while the energy deposited by the proton tracks is calculated from the length of the reconstructed track. The $d E / d x$ of the ionisation tracks and electromagnetic showers is also measured for particle identification purposes and are detailed in Section 6.3 .

Background rejection. The $\nu_{e} \mathrm{CC} 0 \pi-\mathrm{Np}$ events can be further isolated by applying a suite of cuts on kinematic, geometric, and calorimetric variables. The electromagnetic showers initiated by an electron in the final state are isolated with a cut on the $d E / d x$ value and the proton tracks are selected with a cut on the $\chi^{2}$ score of their $d E / d x$ vs. residual range profile. An alternative background-rejection strategy has also been developed using Boosted Decision Trees. These are described in Sections 6.4.1 and 6.4.2. 


\subsubsection{Cosmic-ray rejection}

The hits in the TPC, reconstructed with the procedure described in Chapter 5. are passed to the Pandora framework for pattern recognition.

In order to reject the cosmogenic background, Pandora is first run in cosmic mode over all the reconstructed hits. This reconstruction is track-oriented, and the delta rays are reconstructed as showers and considered as daughters of the closest cosmic muon. The starting point of the reconstructed tracks in this mode is assumed to be the highest $y$ coordinate 97 . These reconstructed high-level objects are fed to a series of cosmic-ray tagging algorithms, which are briefly described below.

Geometry and timing algorithm. This algorithm checks if the reconstructed hits have a time compatible with the drift-time window. If a track or a shower has more than four hits outside the allowed drift-time window, then they are tagged as cosmic rays. Then, the algorithm loops over all the reconstructed objects and tag them if they have a trajectory that enters and exits the TPC borders, within a fiducial volume. The fiducial volume has been chosen by taking into account the magnitude of the space charge effect (Section 4.2).

Flash-matching algorithm. This algorithm takes into account the information provided by the optical system to reject cosmic rays interacting during the readout window. The algorithm first requires the presence of a reconstructed flash during the beam-spill window of $1.6 \mu \mathrm{s}$. Then, for each reconstructed particle a flash hypothesis is built, meaning that we create a distribution of the light collected by each PMT compatible with the charge distribution of the reconstructed particle. The reconstructed particle is tagged as a cosmic ray if its hypothetical flash satisfies two requirements:

1. at least one PMT sees an amount of PE $3 \sigma$ larger than the amount of PE in the flash hypothesis in the same PMT.

2. the $z$ coordinate of the flash hypothesis is not compatible with the $z$ coordinate of the flash observed. 
More details can be found in Section 6.2.4

Anode-Cathode Piercing Tracks algorithm. The coordinate along the drift direction (which in MicroBooNE is the $x$ coordinate) can be reconstructed in a LArTPC only by knowing also the time $t$ when the particle interacted in the detector. The $x$ coordinate is then given by:

$$
x=v_{\mathrm{drift}} t
$$

where $v_{\text {drift }}$ is the drift velocity. In the case of a neutrino interaction, the time $t$ correspond to the beam trigger (plus a definite interval), while for a cosmic ray cannot be known without an external cosmic-ray tagger. However, for the subset of cosmic rays piercing the cathode (or the anode) we will have:

$$
t_{S(E)}-t_{F} \sim t_{C(A)}
$$

where $t_{S}\left(t_{E}\right)$ is the time of the track start point (end point), $t_{F}$ is the time of the flash corresponding to the track, and $t_{C}\left(t_{A}\right)$ is the time corresponding to the position of the cathode (anode). Thus, for each track we loop over all reconstructed flash and we consider the track of cosmic origin if there is a flash which satisfies condition 6.2.

Stopping-muon algorithms. Cosmic muons which enter the TPC and stop in the liquid argon are caused by muons which decayed to a Michel electron while in the TPC. They can be reconstructed as a track (the cosmic muon) and a shower (the Michel electron). Figure 6.3 shows a data event display of the collection plane with a stopping muon.

This topology is similar to the one of an electron neutrino interaction. For this reason, stopping muons represent an important background for our analysis. They are tagged in two ways:

- a series of pattern recognition and calorimetric algorithms try to identify the correct direction of the cosmic muon track (by measuring its energy loss profile) and to verify the presence of a kink in the trajectory, caused by the presence of the Michel electron. 


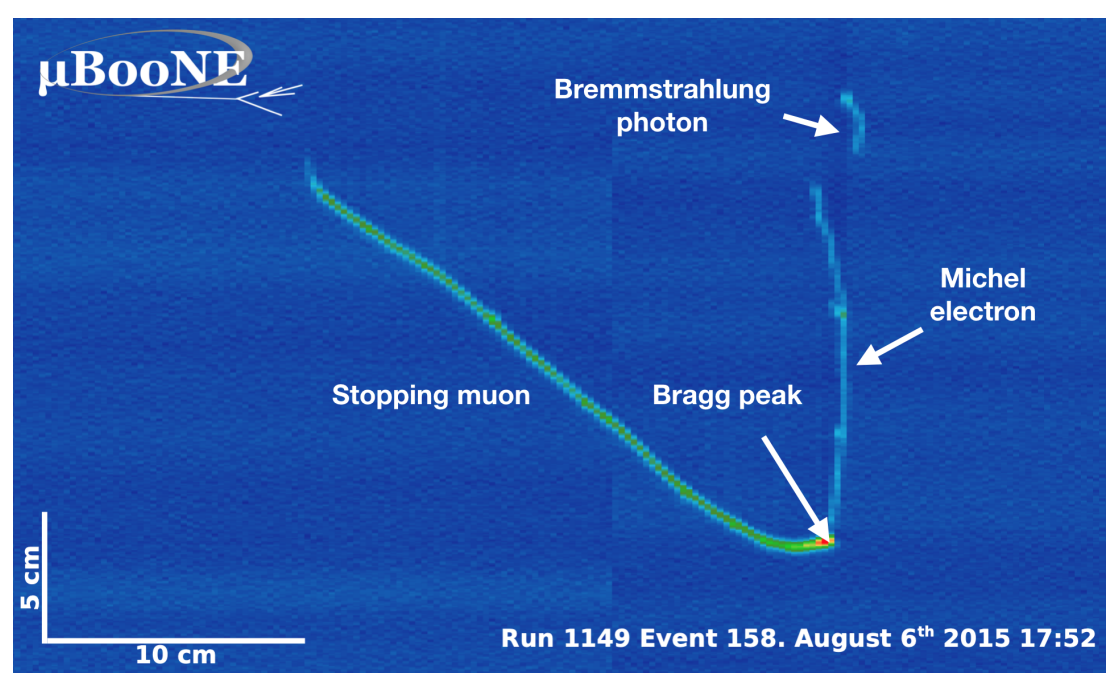

Figure 6.3: Event display of the collection plane with a muon stopping and decaying, producing a Michel electron. Being low-energetic, it resembles a ionisation track and not an electromagnetic shower.

- the multiple Coulomb scattering (MCS) angle of a stopping muon will increase as its momentum decrease, while for through-going muons it is essentially constant 103 . By fitting the track profile with the MCS hypothesis in both direction (up-going and down-going), it is possible to verify if the cosmic muon is stopping in the detector.

The hits associated with the tagged reconstructed objects (and to their daughters) are removed from the collection of reconstructed hits. The remaining hits are then fed to the Pandora neutrino mode reconstruction path, which provides one or more neutrino interaction candidates per event.

\subsubsection{Optical selection}

The optical selection serves two purposes: (1) it ensures that the optical flash which triggered the detector readout is compatible with the neutrino candidates from the Pandora neutrino mode, and (2) it provides a way to discriminate between multiple Pandora neutrino candidate objects (most of which are of cosmic origin and failed the cosmic-removal steps) by selecting the one most compatible with the flash in the optical detection system in time with the beam-gate window.

The optical selection algorithm consists of three major stages: 
1. cuts applied to optical properties of the reconstructed flash object (number of photoelectrons and TPC charge/PMT photoelectrons ratio);

2. cuts on the compatibility of the reconstructed flash with the Pandora neutrino candidate (position of the flash compared with the position of the centre of the collected charge);

3. the Pandora neutrino candidate which is most compatible with the flash is selected using a likelihood method.

The effects of the optical selection have been studied in detail using the $\nu_{e} \mathrm{CC} 0 \pi$ $\mathrm{Np}+$ cosmic Monte Carlo sample, the signal, and the data beam-off sample, the cosmic background.

We first require a reconstructed flash in the optical system within the beam spill window of $1.6 \mu \mathrm{s}$. This requirement selects the $99.6 \%$ of the signal events $\left(\nu_{e} \mathrm{CC} 0 \pi-\mathrm{Np}\right)$ and $18.5 \%$ of the cosmic background events (data beam-off). The reconstructed flash must also have at least $50 \mathrm{PE}$ recorded by the optical system. This is a very conservative requirement and keeps $99.95 \%$ of the signal and $95.2 \%$ of the cosmic background (Figure 6.4).
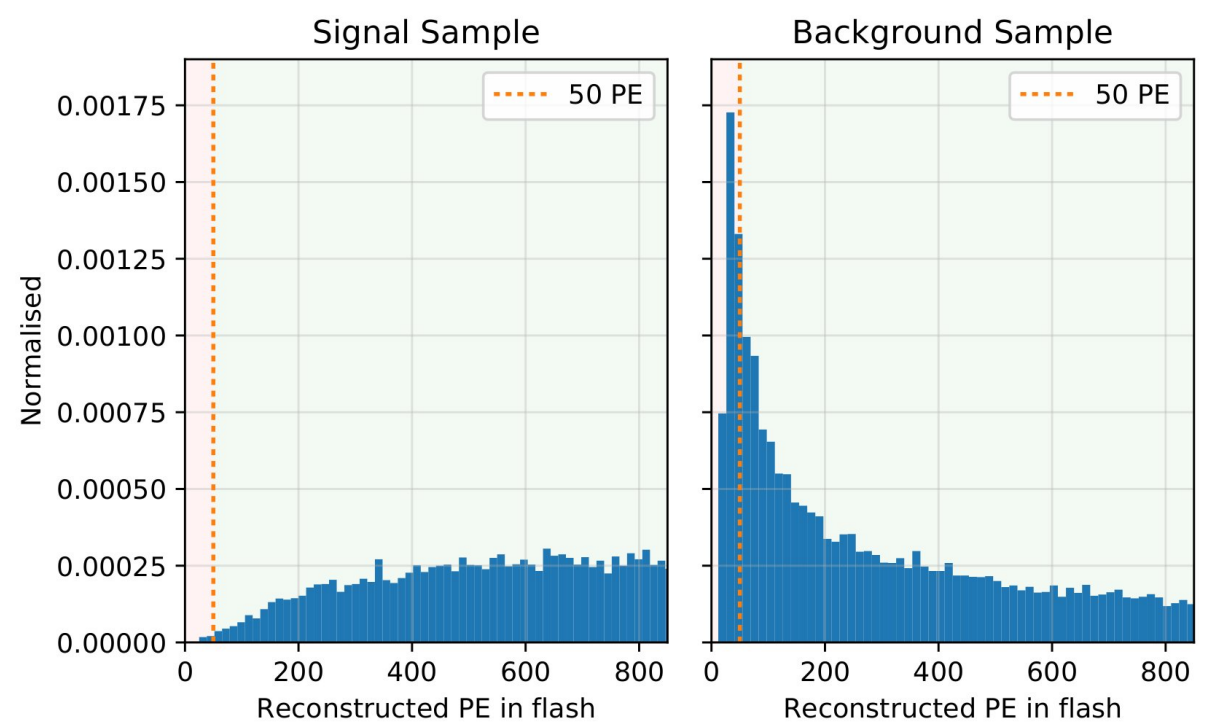

Figure 6.4: Reconstructed PE distribution for signal (left) and cosmic background (right) events. 
These two cuts ensure that the event has a properly reconstructed flash. A flash object has a time and a PE count for each of the 32 PMTs. From this information, two coordinates $z \pm \sigma_{z}$ and $y \pm \sigma_{y}$ are calculated, where the positive $z$ axis corresponds to the beam direction and $y$ coordinate corresponds to the detector height. These two values can be compared with the centre of the deposited charge of the reconstructed neutrino candidate in the TPC. This comparison has the implicit assumption that the light will be emitted in the same relative fraction as the charge deposited by the particles in the final state. This is not completely correct since the amount of scintillation light produced per deposited energy unit depends on the particle. Nevertheless, the coarse resolution given by the PMT grid allows to use this approximation.

A cut of $105 \mathrm{~cm}$ is placed on the difference between the reconstructed flash position and the centre of the deposited charge on the $z$ axis. This cut keeps at least one neutrino candidate in $98.1 \%$ of the signal events, and it removes all candidates in $20 \%$ of the background events (Figure 6.5). Similar cuts are placed taking into account the width of the flash and its position on the $y$ axis.
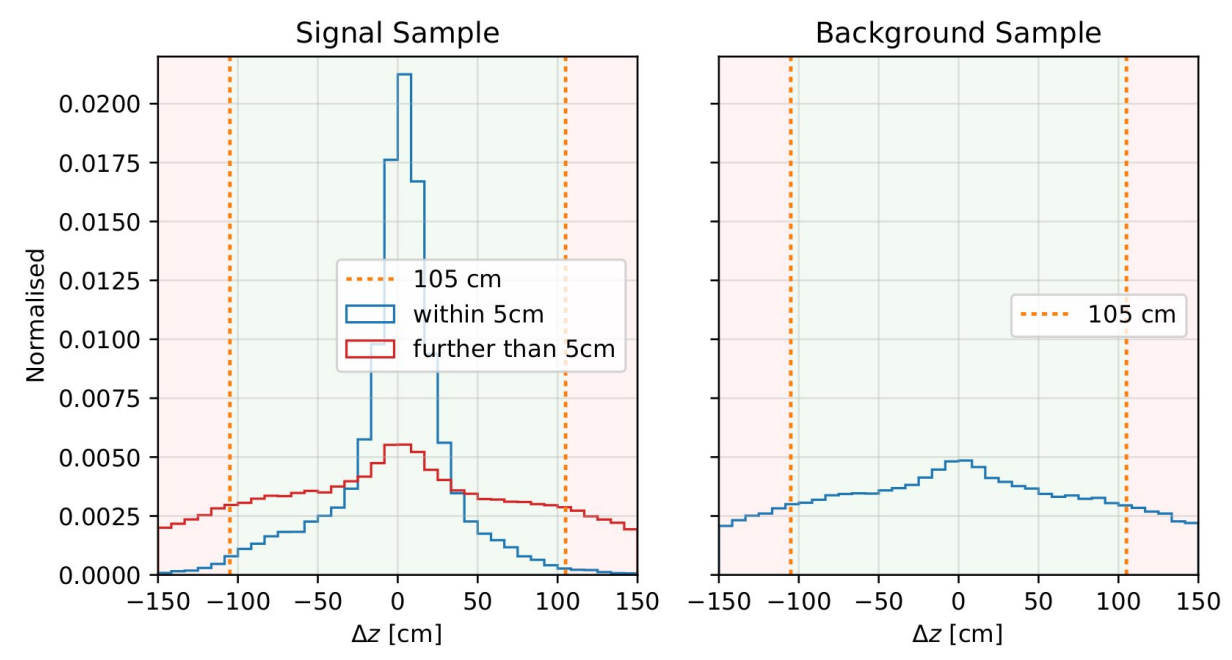

Figure 6.5: Distribution of the distance between the reconstructed flash position and the centre of the deposited charge on the $z$ axis for signal (left) and cosmic background (right). The within $5 \mathrm{~cm}$ and further than $5 \mathrm{~cm}$ categories refers to the distance between the reconstructed neutrino vertex and the true neutrino vertex.

The last rectangular cut exploits the fact that several neutrino candidates 
reconstructed by Pandora originate from remnants of cosmic activity which were not tagged by the cosmic-removal algorithms, since they will look like small isolated showers or tracks. Those neutrino candidates often consist in a small amount of fragmented charge, incompatible with the brightness of the flash. Placing a very conservative cut at 3.0 on the ratio between the charge in the collection plane associated to the neutrino candidate and the number of PEs reduces the signal events with a properly reconstructed flash by $1.7 \%$, while removing all candidates in $15.4 \%$ of the background events (Figure 6.6).
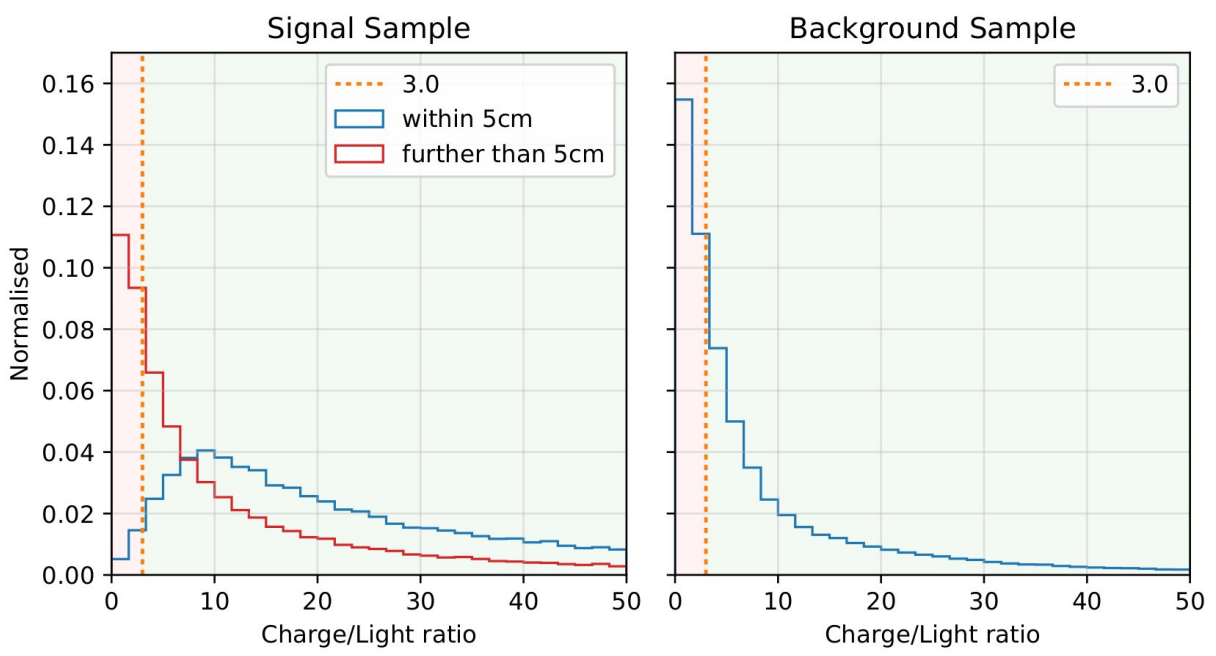

Figure 6.6: Distribution of the ratio between the charge in the collection plane associated to the neutrino candidate (in ADC counts) and the number of PEs collected by the PMTs. The within $5 \mathrm{~cm}$ and further than $5 \mathrm{~cm}$ categories refers to the distance between the reconstructed neutrino vertex and the true neutrino vertex.

After these rectangular cuts it is still possible to have more than one reconstructed neutrino candidate in the event. A more sophisticated flash-matching procedure allows to choose the one that best matches the collected light:

1. for every neutrino candidate, a spatial distribution of deposited charge is measured;

2. the spatial distribution of the deposited charge is translated into an estimation of the emitted scintillation light. These scintillation photons are then propagated towards the PMTs to construct a flash hypothesis using only TPC information; 
3. the flash-matching algorithm compares the reconstructed flash object as seen by the PMTs with the hypothetical flash for every reconstructed neutrino candidate and picks the best-matching candidate. This selection is achieved through a binned likelihood of the PMT spectrum.

An example of this procedure for a Monte Carlo generated $\nu_{e}$ event with 4 neutrino candidates is given in Figure 6.7.

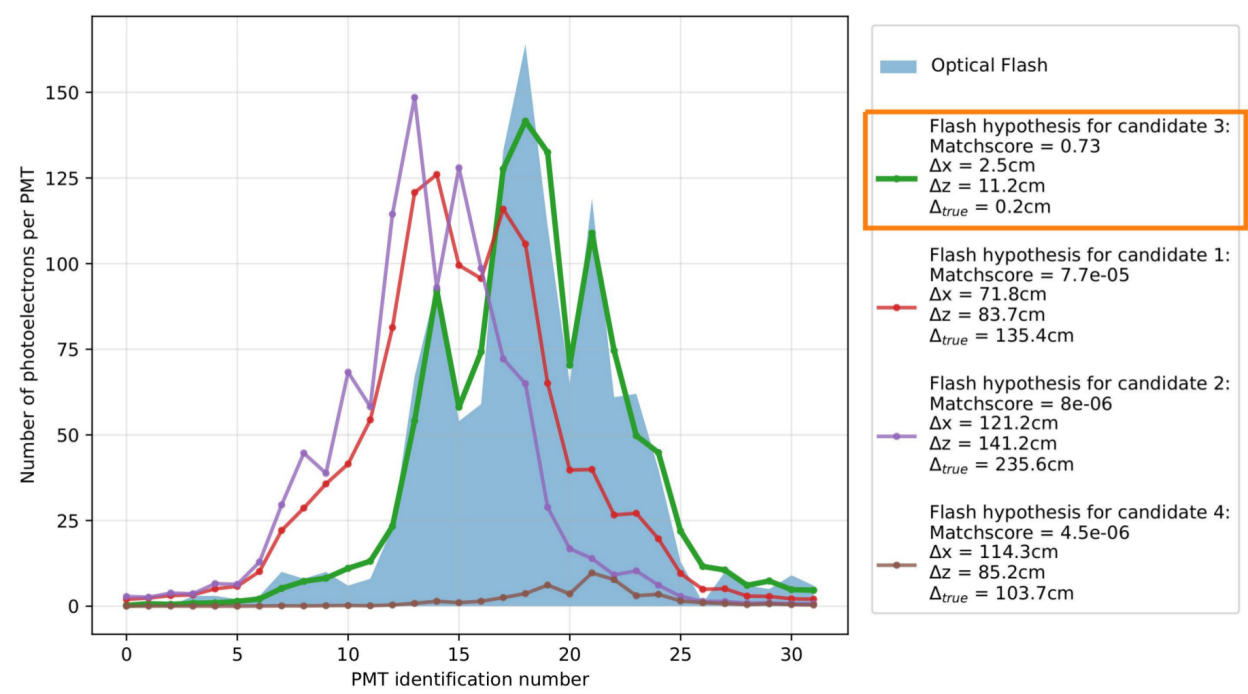

Figure 6.7: An example of flash-matching. The event has 4 neutrino candidates and we make a flash hypothesis for each one, shown in red, purple, green, and brown. The observed flash corresponds to the filled blue area. A minimum binned likelihood is calculated, varying the $x$ position of the interaction. The match score is the inverse of the likelihood. The candidate with the highest match score is chosen as neutrino interaction candidate (the green one in this case).

\subsubsection{Topological pre-selection}

A perfect reconstruction of a $\nu_{e} \mathrm{CC} 0 \pi$-Np event in a LArTPC will produce as many reconstructed tracks as the number of protons above the detection threshold in the final state and a single reconstructed shower (the electron), sharing a common vertex. However, mis-reconstruction and mis-classification issues can significantly lower the selection efficiency. The current status of the event reconstruction, which depends on the properties of the event (e.g. the number of hits 97]), affects the efficiency of selecting these events. For example, the presence of dead or unresponsive wires can affect the reconstruction by causing the splitting of an ionisation track or an 

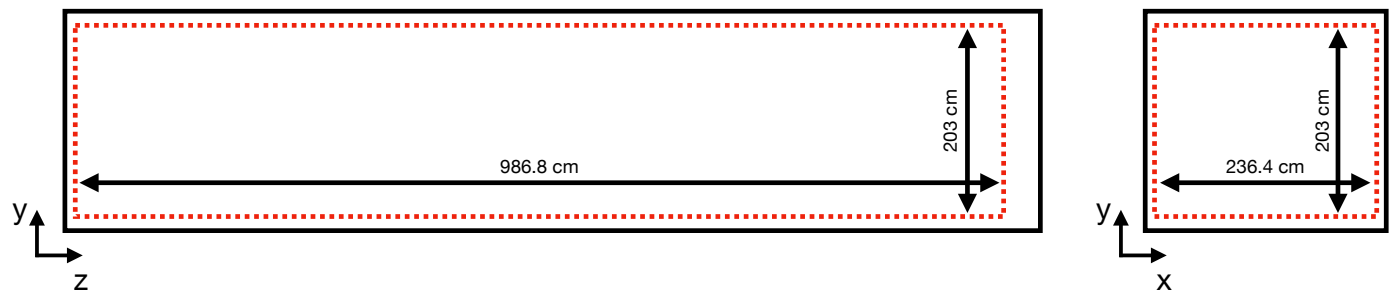

Figure 6.8: Schematic of the fiducial volume used in this analysis. The solid line corresponds to the TPC borders and the dashed red line corresponds to the fiducial volume borders.

electromagnetic shower into two distinct reconstructed objects. Also, the selection currently implemented relies on the classification of the reconstructed objects as track-like or shower-like, a separation that contains an inherent inefficiency, especially when the number of reconstructed hits is low.

In order to maximise our efficiency we currently require (1) at least one track and at least one shower sharing a common vertex, or (2) at least two showers sharing a common vertex, to account for proton mis-classification as a shower-like object. This is because it is much more common to have protons classified as showers than electrons classified as tracks. For these cases we measure the $\chi^{2}$ score of the $d E / d x$ vs. residual range profile of the reconstructed objects in the proton hypothesis, as described in Section 6.3.7. The object with the lowest proton $\chi^{2}$ score is classified as a track, while the other ones remain classified as showers.

\subsubsection{Minimum reconstruction quality requirements}

A minimal set of cuts is applied to the selected events, in order to ensure that they are well reconstructed. First, to avoid border effects, the reconstructed neutrino vertex, the start point of the reconstructed showers and the start and end points of the reconstructed tracks are required to lie within a fiducial volume. Our fiducial volume cut is $10 \mathrm{~cm}$ from each side on the $x$ axis, $15 \mathrm{~cm}$ from each side on the $y$ axis, and $10 \mathrm{~cm}(40 \mathrm{~cm})$ from the upstream (downstream) side on the $z$ axis (Figure 6.8). The fiducial volume corresponds to $76.4 \%$ of the total TPC volume.

Since electromagnetic showers develop mainly in the forward direction with respect to the beam, the asymmetric cut on the $z$ axis (which corresponds to the 


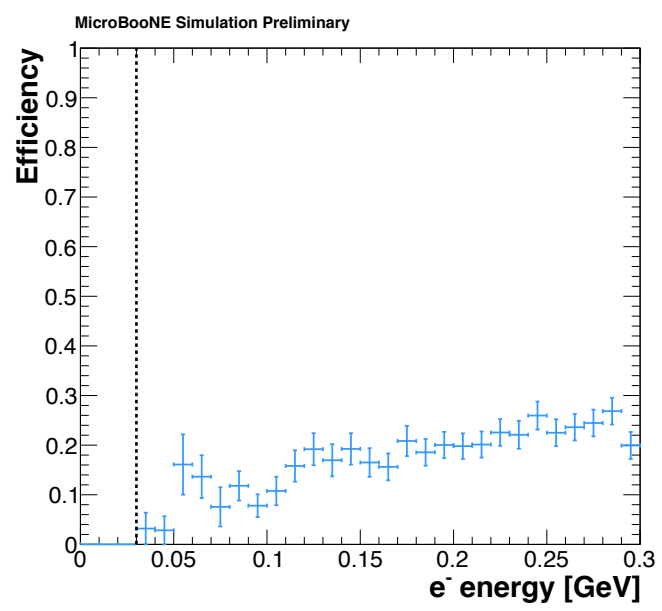

(a) Electron efficiency.

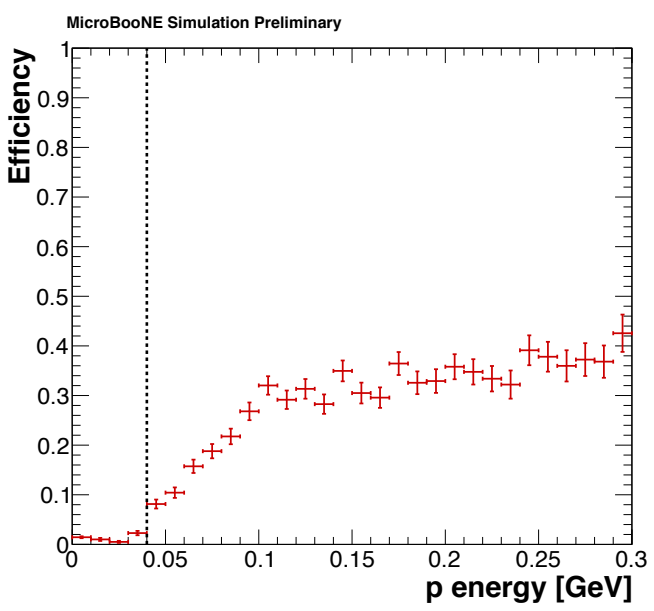

(b) Proton efficiency.

Figure 6.9: $\nu_{e} \mathrm{CC} 0 \pi-\mathrm{Np}$ selection efficiency on single particles as a function of the true electron (left) and proton (right) kinetic energy. The dashed lines correspond to the threshold applied at truth level.

beam direction) helps to reject non-fully contained events which begin too close to the downstream end of the TPC. We also require, for each event, (1) at least 5 hits in the three planes associated to shower-like objects, (2) at least 5 hits in the three planes associated to track-like objects, and (3) at least one hit in every plane.

\subsubsection{Selection efficiency and purity}

The selection efficiency of our algorithm is obtained by calculating the fraction of events selected in the $\nu_{e} \mathrm{CC} 0 \pi-\mathrm{Np}+$ cosmic Monte Carlo sample, where the true neutrino vertex, the start and end points of the protons, and the start point of the electron are fully contained in the fiducial volume.

In order to understand what energy thresholds are appropriate for reconstruction in the TPC, dedicated studies have been performed on proton tracks and electron showers, using the $\nu_{e} \mathrm{CC} 0 \pi-\mathrm{Np}+$ cosmic Monte Carlo sample, shown in Figure 6.9. We have found that we have no efficiency for reconstructing and classifying protons with a kinetic energy below $40 \mathrm{MeV}$ and electrons, photons, and charged pions with a kinetic energy below $30 \mathrm{MeV}$ following these optical, topological, and minimum quality pre-selections. Therefore, these energy thresholds are applied to the simulations to allow a fair comparison with the reconstructed particles. 


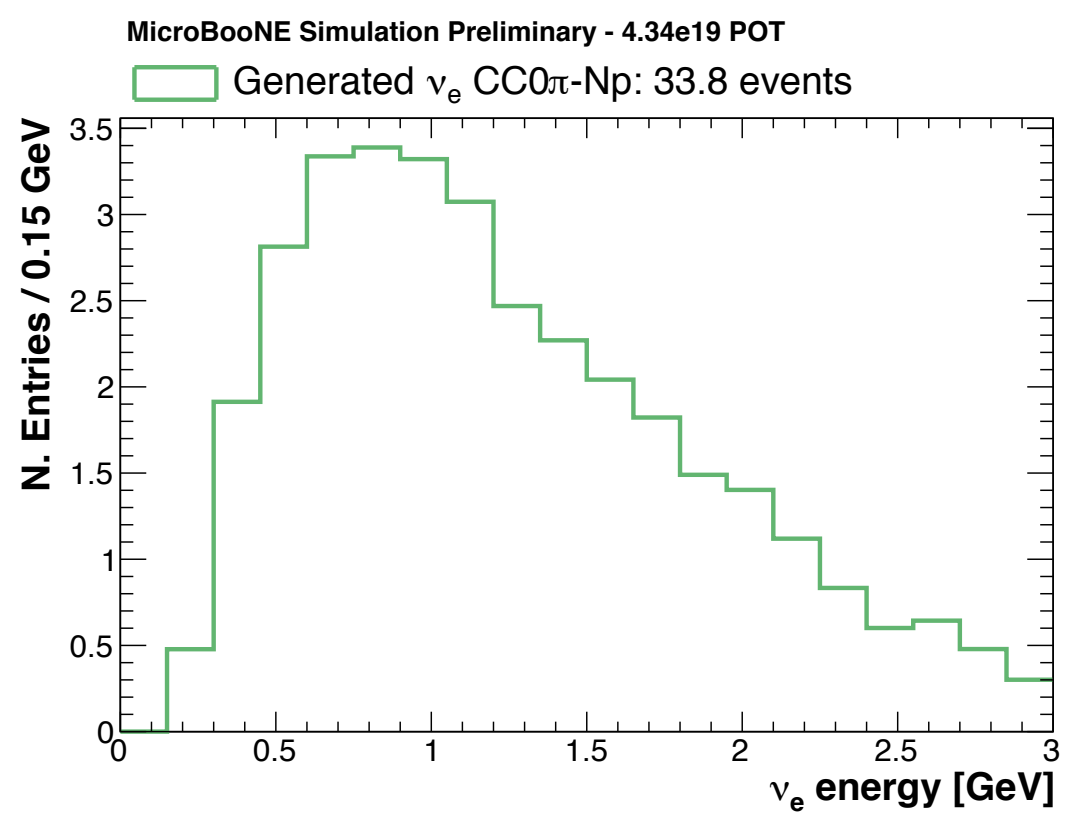

Figure 6.10: Simulated $\nu_{e} \mathrm{CC} 0 \pi-\mathrm{Np}$ true neutrino energy spectrum in the $0-3 \mathrm{GeV}$ range. Each true proton (true electron) in the final state is required to have a kinetic energy larger than $40 \mathrm{MeV}(30 \mathrm{MeV})$.

Our overall $\nu_{e} \mathrm{CC} 0 \pi-\mathrm{Np}$ selection efficiency $\epsilon$ is defined as:

$$
\epsilon=\frac{\mathrm{N} . \text { of selected } \nu_{e} \mathrm{CC} 0 \pi-\mathrm{Np} \text { events }}{\mathrm{N} . \text { of generated } \nu_{e} \mathrm{CC} 0 \pi-\mathrm{Np} \text { events }}
$$

where each selected event must pass the optical selection, satisfy the topology and minimum quality requirements, and not being vetoed by the independent $\mathrm{CC} \nu_{\mu}$ selection module.

The true neutrino energy spectrum of the simulated $\nu_{e} \mathrm{CC} 0 \pi$-Np events in the $[0,3] \mathrm{GeV}$ range is shown in Figure 6.10 .

Figure 6.11 shows the efficiency as a function of the true neutrino energy. The systematic uncertainties related to the cross-section and the neutrino beam flux, described in Chapter 7 are also included. The inner error bars represent the Monte Carlo statistical uncertainty, while the outer error bars are obtained summing in quadrature the statistical and the systematic uncertainties. The statistical uncertainty $\delta \epsilon$ corresponds to the binomial error, since the application of a selection can be considered a binomial process 104:

$$
\delta \epsilon=\frac{1}{N} \sqrt{k(1-k / N)}
$$


MicroBooNE Simulation Preliminary

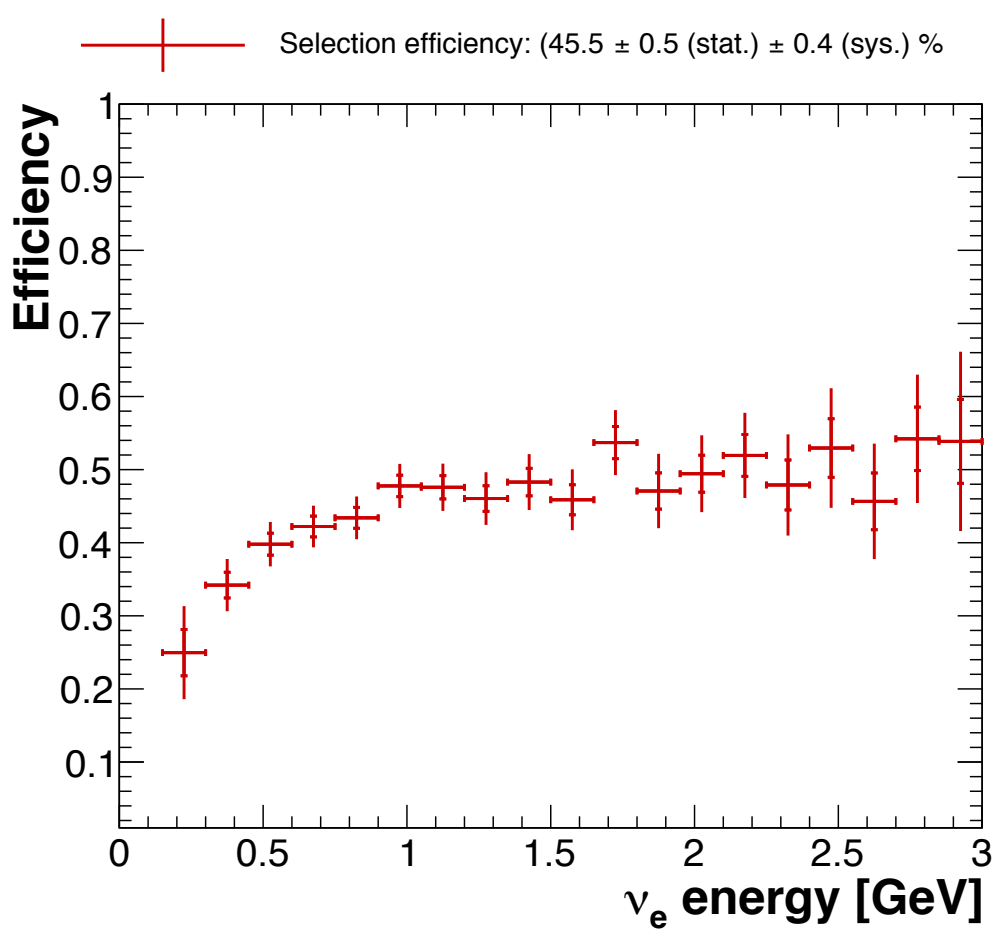

Figure 6.11: $\nu_{e} \mathrm{CC} 0 \pi-\mathrm{Np}$ selection efficiency as a function of the true $\nu_{e}$ energy. Each true proton (true electron) in the final state is required to have a kinetic energy larger than $40 \mathrm{MeV}(30 \mathrm{MeV})$. The inner error bars represent the Monte Carlo statistical uncertainty, while the outer error bars are obtained summing in quadrature the statistical and the systematic uncertainties.

where $k$ is the number of selected events and $N$ is the total number of events.

As expected, the efficiency increases with the neutrino energy, since high-energy neutrino interactions correspond in general to a larger number of hits in the TPC and the Pandora framework reconstruction performances increase with the number of reconstructed hits 97 .

\section{Inefficiencies breakdown}

Our current selection algorithm can fail for several reasons: in particular, we could have problems in the classification, such as an electron classified as a track-like object, or particles not reconstructed at all. We identified eight main causes for our selection inefficiency, whose contributions have been estimated with the same simulated sample described in Section 6.2.7. The different contributions can be 
visualised in Figure 6.12 and are described below.

Quality cuts (8.5\%). The selected neutrino candidate does not satisfy our minimum quality requirements, such as the number of shower hits and the number of track hits (Section 6.2.6).

CC $\nu_{\mu}$ selected $(\mathbf{4 . 3 \%})$. The event is tagged as a CC $\nu_{\mu}$ candidate by an independent selection module, described in 102 .

Not contained $\mathbf{( 1 0 . 1 \% )}$. One of the reconstructed tracks or the starting point of one of the reconstructed showers is not contained in the fiducial volume. As expected, this fraction increases with the neutrino energy.

Cosmic selected (7.9\%). The selected neutrino candidate has one or more reconstructed objects of cosmic origin.

1 shower $(3.5 \%)$. The selected neutrino candidate has only one associated reconstructed shower and no track objects.

No showers (13.7\%). The selected neutrino candidate has only reconstructed $\operatorname{track}(\mathrm{s})$ associated. This is the largest contribution to the inefficiency, especially at low energies, since low-energy electrons are very challenging to reconstruct and classify as showers.

No flash $\mathbf{( 5 . 7 \% )}$. The flash collected by the optical system does not satisfy our requirements, such as the minimum number of $\mathrm{PE}$, location of the flash and flash hypothesis (Section 6.2.4).

No data products $\mathbf{( 0 . 7 \% )}$. The Pandora pattern recognition did not identify any neutrino candidate.

Figure 6.12 shows a stacked histogram of the true neutrino energy for the $\nu_{e} \mathrm{CC} 0 \pi-\mathrm{Np}$ generated events, divided into the categories described above. The events without a reconstructed shower are the dominant inefficiency at low energy. Low-energy showers correspond in general to a smaller number of reconstructed 


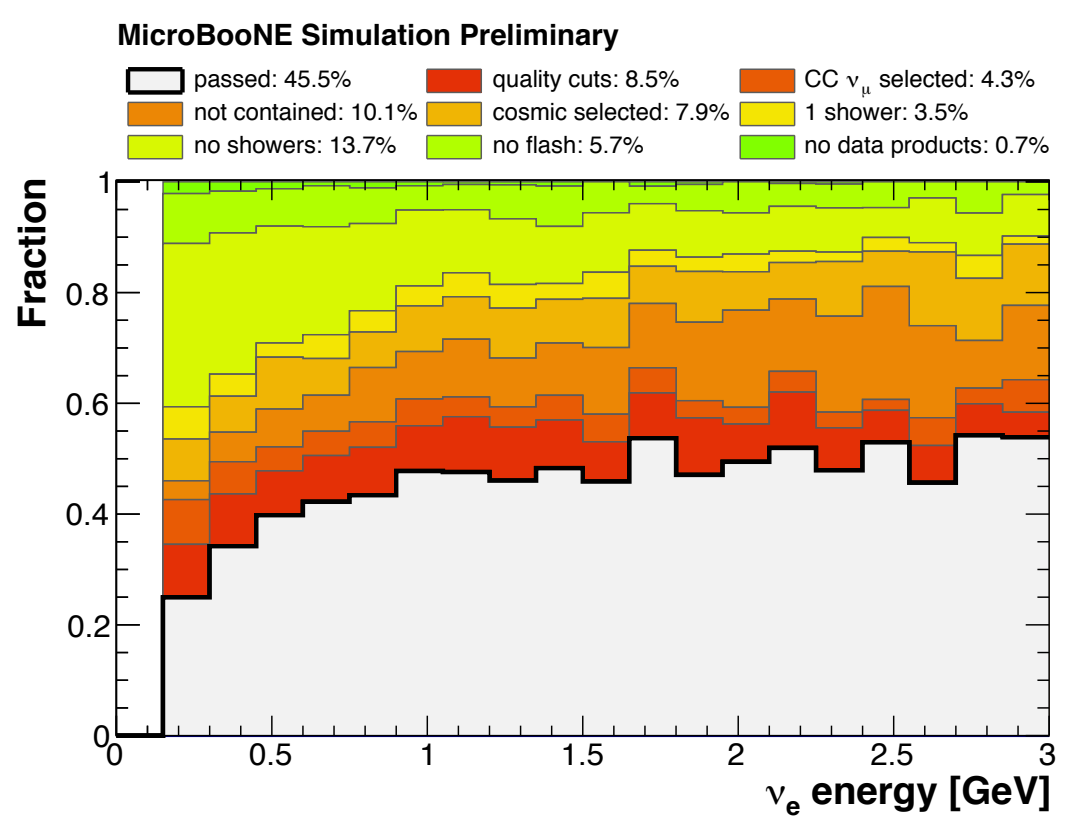

Figure 6.12: Stacked histogram of generated events as a function of the true neutrino energy, categorised into correctly identified signal events in grey (passed) and different reconstruction or identification failure modes in colour.

hits, which makes the pattern recognition more challenging [97]. The fraction of events where at least one reconstructed object is not contained in the detector (not contained in the legend) increases with the energy. This is expected, since the probability to have large electromagnetic showers split in two or more object increases, and these objects can have a vertex reconstructed outside the fiducial volume. The passed category (filled grey histogram) corresponds to the efficiency plot shown in Figure 6.11

For more detailed information, the selection outcomes as a function of the lepton true angular variables $\theta$ and $\phi$ are shown in Figure 6.13. The efficiency is mostly constant as a function of the azimuthal angle $\phi$, while the fraction of events without reconstructed showers increases as a function of the $\theta$ angle. This is because backwards-going electrons are more difficult to reconstruct, since they often have lower energy, and the pattern recognition tends to group the electron hits together with the forward-going proton track. 


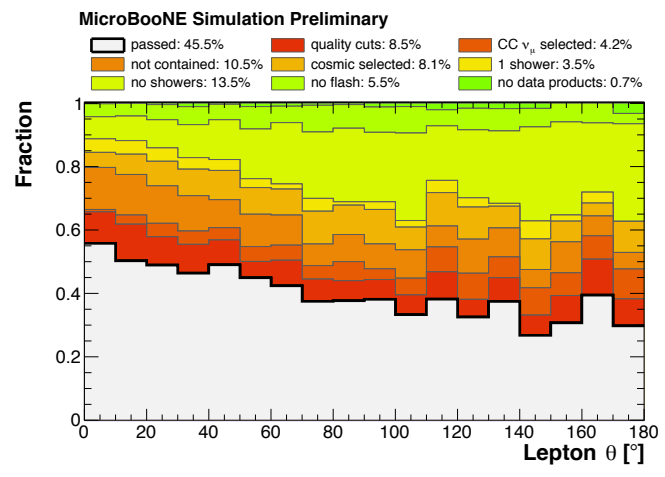

(a) $\theta$ efficiency.

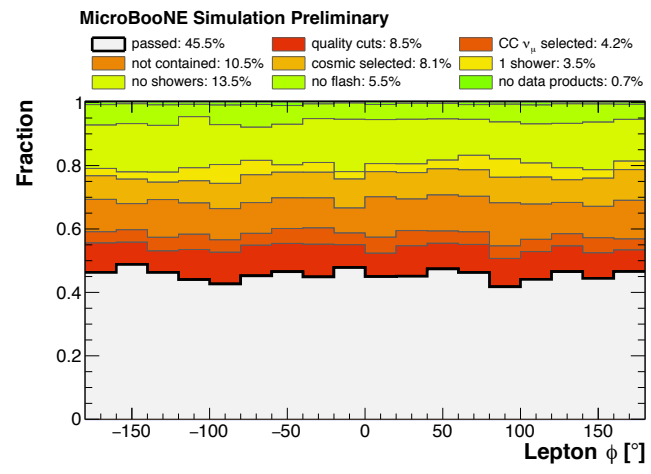

(b) $\phi$ efficiency.

Figure 6.13: Stacked histogram of generated events as a function of the electron $\theta$ (left) and $\phi$ (right) angles, categorised into correctly identified signal events (passed) in grey and different reconstruction or identification failure modes in colour.

\subsubsection{Selection performances in BNB events}

The previous selection efficiency results were performed on a dedicated $\nu_{e} \mathrm{CC} 0 \pi-\mathrm{Np}$ + cosmic sample. We now look at the selection performances when analysing events coming from the complete set of the events acquired by MicroBooNE from the Booster Neutrino Beam, including all flavours of neutrinos with different fraction (see Figure 4.6). In the $B N B+$ cosmic sample every event will have at least one neutrino interacting in the cryostat volume and triggering the detector, plus all the cosmic rays hitting the detector in the same readout window. In the data, however, this is not always true, since the detector can be triggered also by a cosmic ray producing a flash in the optical system during the beam window, without necessarily having a neutrino interaction. In order to estimate this background component, defined as in-time cosmic rays, we have used the data sample collected with the data EXT trigger. The background caused by the neutrino interactions happening outside the cryostat has been evaluated using the dirt sample. We divide the selected events (signal and background) into 8 categories:

\section{Signal}

Beam intrinsic $\nu_{e} \mathbf{C C} 0 \pi-\mathrm{Np}$ : charged-current $\nu_{e}$ neutrino interaction, at least one proton $(\mathrm{N}>1)$, one electron, and no other visible particles above 
detection threshold. This category represents the signal of our analysis.

\section{Backgrounds}

Beam intrinsic $\nu_{e} \mathbf{C C}$ : charged-current $\nu_{e}$ neutrino interaction that is not $\nu_{e} \mathrm{CC} 0 \pi-\mathrm{Np}$ or where the electron or protons were below the detection threshold defined above.

Beam intrinsic $\nu_{\mu}$ : charged-current $\nu_{\mu}$ neutrino interaction.

Beam intrinsic NC: neutral current neutrino interaction (both $\nu_{\mu}$ and $\nu_{e}$ ).

Outside fiducial volume: neutrino interaction which occurs outside the fiducial volume, but with one or more final-state particles inside in the fiducial volume.

Cosmic contaminated: neutrino interaction candidate with at least a cosmogenic track or shower, attached to a correctly reconstructed neutrino candidate.

Cosmic: cosmic ray interaction happening in the same readout window is mistakenly chosen instead of the neutrino interaction in the event.

Data beam-off : event with no neutrino interaction, but where a cosmic-ray interaction in time-coincidence with the beam-gate window triggered the event, and activity was selected as a neutrino candidate.

Table 6.1 shows a summary of the selection algorithm results, with the corresponding number of events for each category.

At this point, all selected events have a neutrino interaction candidate with one or more tracks and one or more showers associated with the interaction vertex. Since we use two different methods to measure the energy of the tracks and the energy of the showers (see Section 6.3), it is necessary to verify the agreement between the shower multiplicity and track multiplicity distributions in data and Monte 
Table 6.1: Summary of the selection results, showing the contribution of each event category, for a MicroBooNE exposure of $4.34 \times 10^{19}$ POT. Efficiency uncertainties are statistical only. For the Cosmic contaminated category the number of generated events correspond to the number of neutrino interactions inside the cryostat. For the Cosmic category, it corresponds to the total number of simulated neutrino interactions, both inside and outside the cryostat.

\begin{tabular}{lrrr}
\hline Category & Generated & Selected & Efficiency [\%] \\
\hline$\nu_{e}$ CC0 $\pi$-Np (signal) & 33.8 & 15.4 & $45.5 \pm 0.5$ \\
$\nu_{e}$ CC & 39.5 & 15.4 & $39.0 \pm 0.5$ \\
Beam intrinsic $\nu_{\mu}$ & 10905.9 & 488.9 & $4.5 \pm 0.2$ \\
Beam intrinsic NC & 3532.8 & 329.5 & $9.3 \pm 0.2$ \\
Outside fid. vol. & 36634.6 & 79.0 & $0.2 \pm 0.1$ \\
Data off-beam & 123070.2 & 1593.4 & $1.3 \pm 0.1$ \\
Cosmic contaminated & 14706.4 & 376.1 & $2.5 \pm 0.1$ \\
Cosmic & 51356.1 & 489.4 & $0.8 \pm 0.1$ \\
\hline
\end{tabular}

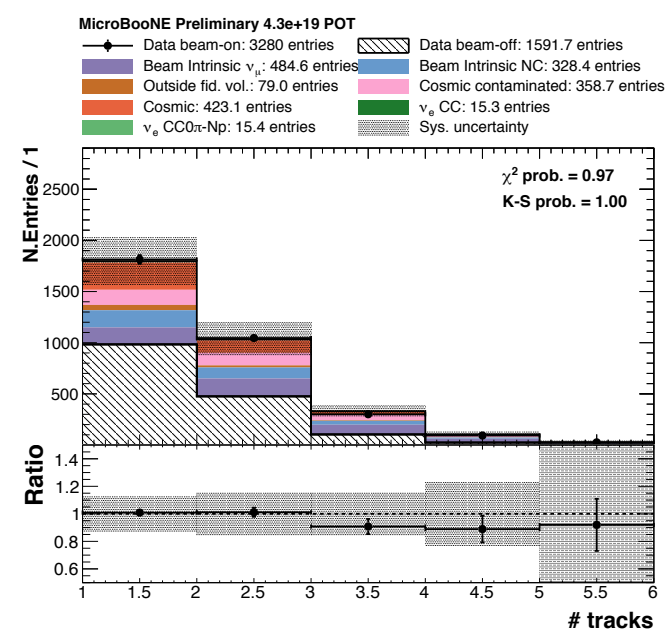

(a) Track multiplicity.

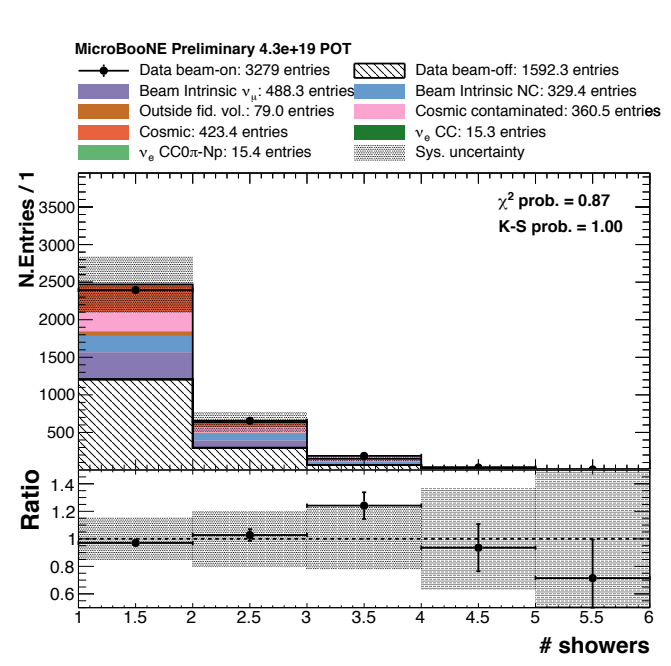

(b) Shower multiplicity.

Figure 6.14: Distributions of the track and shower multiplicities in data and Monte Carlo simulation.

Carlo. Figure 6.14 shows that the two distributions agree within the systematic uncertainties, whose evaluation is described in Chapter 7 .

The agreement between data and simulation is also verified in the angular distributions of the reconstructed showers objects, shown in Figure 6.15. As expected, the neutrino distributions are mostly constant as a function of the azimuthal angle $\phi$ and peaked at low inclination angle $\theta$ values, since the interactions are mostly forward going. The inclination angle $\theta$ distribution agrees within the uncertainties 
both for shape and normalisation. The azimuthal angle $\phi$ distribution shows a slight disagreement around $\phi=0^{\circ}$ and $\phi= \pm 180^{\circ}$. This is caused by an imprecise signal simulation that predominantly affects tracks moving exactly towards or away from the anode [105]. This effect is taken into account in the Dynamic Induced Charge detector systematic sample (Section 7).

Figure 6.16 shows the angular distributions classified according to the primary particle that generated the shower (in the case of a Michel electron the shower is placed in the muon category). Each entry in the histogram corresponds to a reconstructed shower, so it is possible to have more than one entry per event. As expected, the $\theta$ distribution is peaked at low angles, since neutrino interactions are mostly forward going. The $\phi$ distribution is mainly flat for neutrino-induced particles (electrons, photons) and with two peaks at $\pm 90^{\circ}$ for mostly-vertical cosmicinduced particles (mainly muons). In this case, the data points correspond to the bin-by-bin statistical subtraction of the beam-on and beam-off entries.

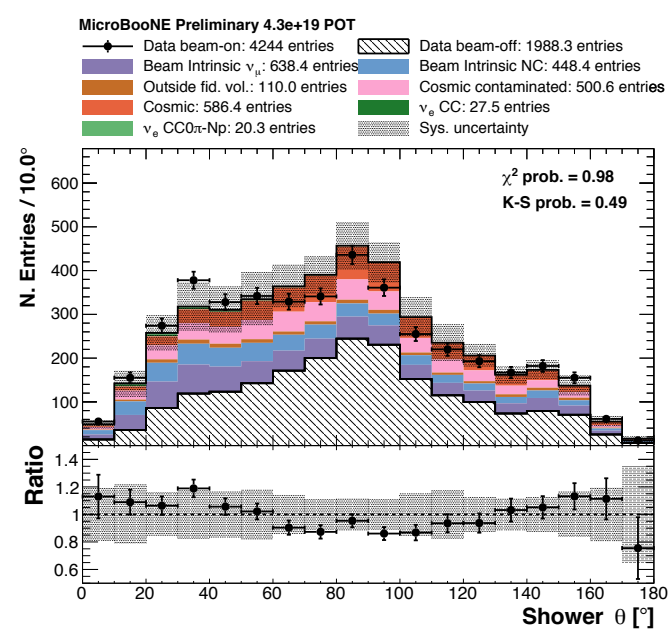

(a) Inclination angle $\theta$.

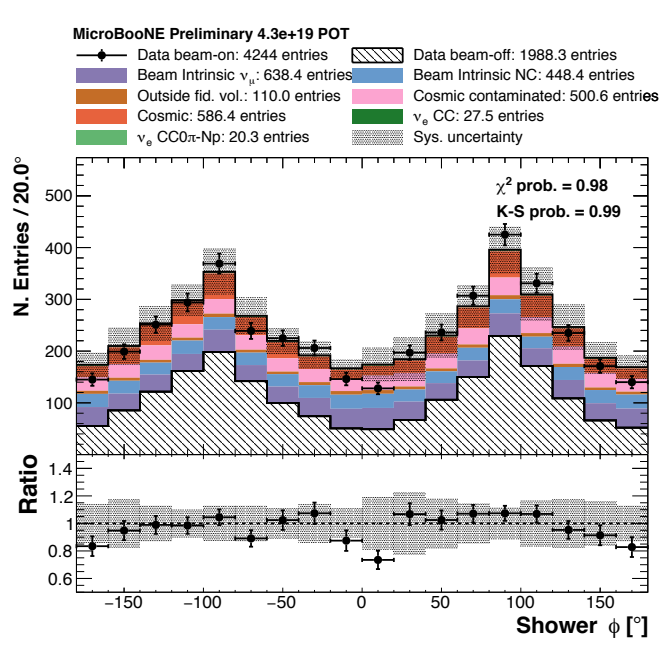

(b) Azimuth angle $\phi$.

Figure 6.15: Distributions of the inclination angle $\theta$ and the azimuthal angle $\phi$ of the reconstructed showers in the selected events for each event category. The black points represent the data with statistical uncertainties. The coloured stacked histograms represent the simulated events, with the hatched histogram corresponding to the data beam-off sample. The shaded area represents the systematic uncertainty. The bottom part of the plot shows the ratio between the data beam-on events and the stacked histograms.

A small fraction of the data events was also visually inspected: Figure 6.17 shows three event displays of data events compatible with a $\nu_{e} \mathrm{CC} 0 \pi-\mathrm{Np}$ interaction. 


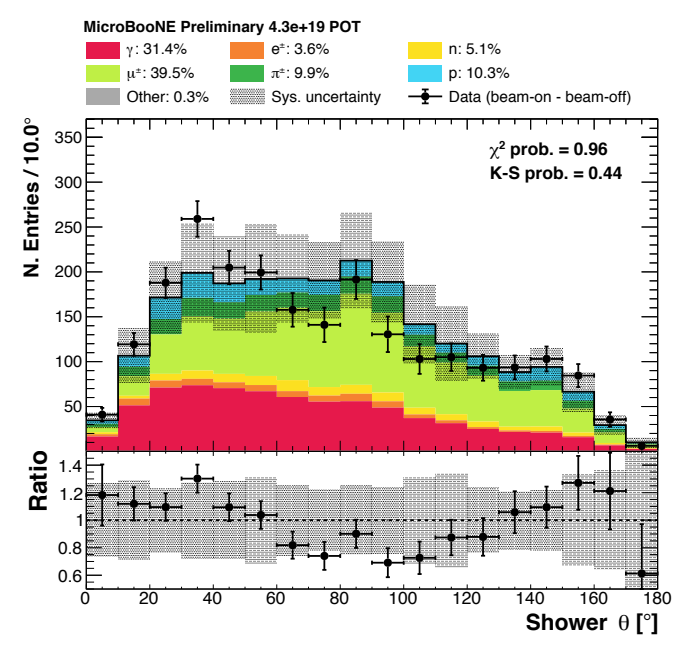

(a) Inclination angle $\theta$.

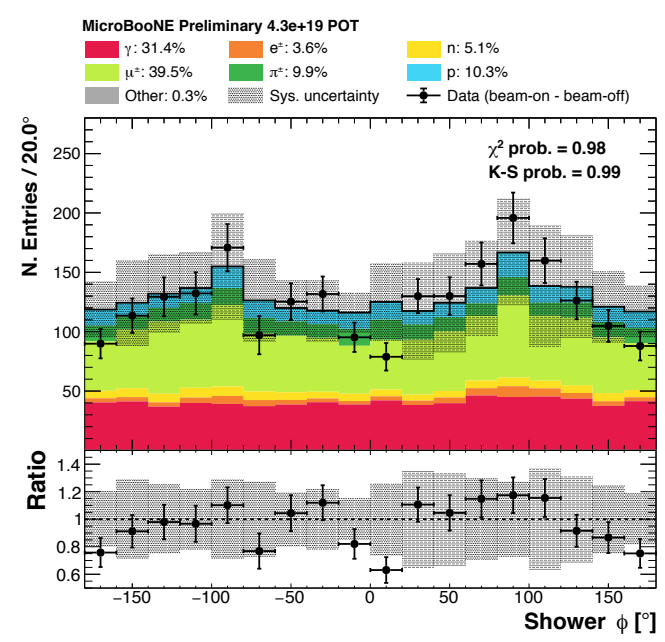

(b) Azimuth angle $\phi$.

Figure 6.16: Distributions of the inclination angle $\theta$ and the azimuthal angle $\phi$ of the reconstructed showers, classified according to the primary particle that generated them. The black points represent the statistically subtraction of the data beam-off events from the data beam-on events. The coloured stacked histograms represent the simulated events. The shaded area represents the systematic uncertainty. The bottom part of the plot shows the ratio between the statistical subtraction and the stacked histograms.

\subsection{Calorimetry}

\subsubsection{Scope of the energy reconstruction}

In this analysis we restrict ourselves to the measurement of the deposited energy in the TPC of the visible particles in the final state of the $\nu_{e} \mathrm{CC} 0 \pi-\mathrm{Np}$ neutrino interaction. We will not attempt to reconstruct the interacting neutrino energy. Our signal has in its final state, by definition, one electron and at least one proton, with no other visible particles. The energy of the showers, expected to be from an electron, is measured by converting the reconstructed charge of all the shower-like objects into deposited energy, as described in Section 6.3.2. The energy of the tracks, expected to be from protons instead, can be measured by converting the track length of the reconstructed tracks into deposited energy, using the tabulated stopping power of protons in the liquid argon, with the procedure described in Section 6.3.3. Thus, object classification issues, such as proton classified as showers or electrons classified as tracks, can have a direct effect on the energy reconstruction. 


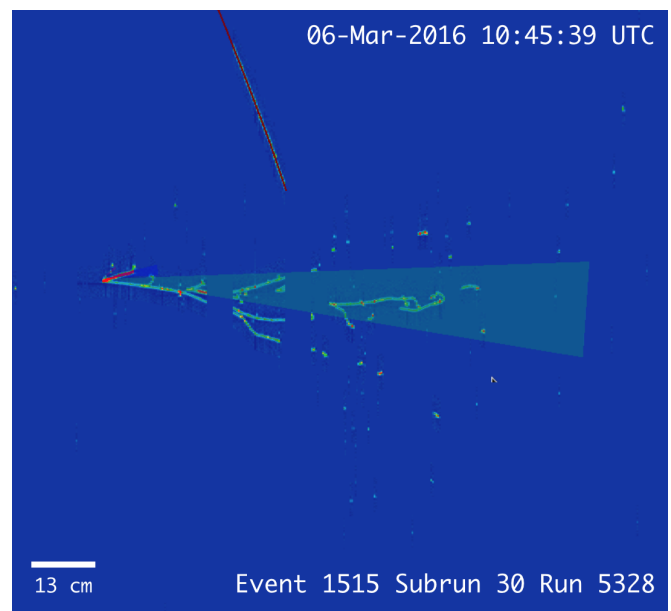

(a) Event 1515, Subrun 30, Run 5328

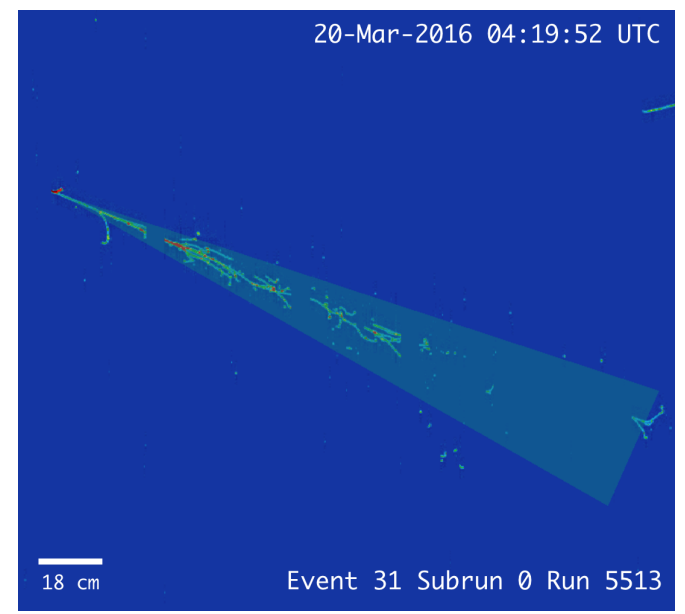

(b) Event 31, Subrun 0, Run 5513

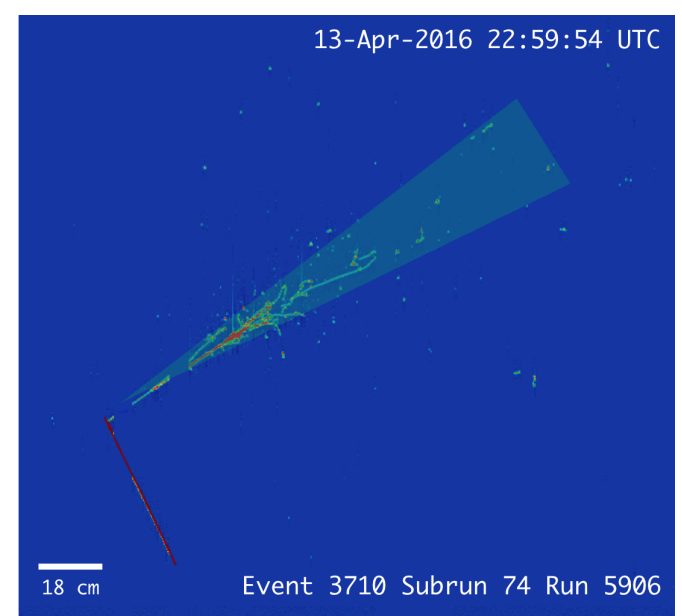

(c) Event 3710, Subrun 74, Run 5906

Figure 6.17: Event displays of the collection plane of three $\nu_{e}$-like data events selected by our algorithm. The gaps are caused by the presence of dead or unresponsive wires. The red lines correspond to reconstructed track-like objects and the green cones correspond to reconstructed shower-like objects.

The total reconstructed energy corresponds to the sum of the reconstructed energies, corrected by the calibration factors calculated below, and is referred to as $E_{\text {corr }}$. This quantity is then compared with the total kinetic energy of the particles above detection thresholds and corrected by a calibration factor to obtain an estimate of the deposited energy $E_{\text {deposited }}($ Section 6.3.4). 


\subsubsection{Electron energy reconstruction and calibration}

The reconstructed energy $E_{\text {reco }}^{e}$ of a shower-like object is measured by converting the charge of the associated hits into deposited energy in the TPC. It is calculated by multiplying the reconstructed charge $\left(e_{\text {reco }}^{-}\right)$from hits associated with the reconstructed shower by the calibration factor 106 :

$$
\frac{E_{\text {reco }}^{e}(\mathrm{MeV})}{e_{\text {reco }}^{-}}=1.01 \frac{e^{-}}{e_{\text {reco }}^{-}} \times \frac{23.6 \mathrm{eV}}{e^{-}} \times 10^{-6} \frac{\mathrm{MeV}}{\mathrm{eV}} \times \frac{1}{R}=3.85 \times 10^{-5},
$$

where:

- the correction factor $1.01 \frac{e^{-}}{e_{\text {reco }}^{-}}$is obtained measuring the true number of collected electrons $e^{-}$on the wires using a sample of stopping muons, fitting the $d E / d x$ vs. residual range to values for argon as tabulated by the PDG 19 ;

- $\frac{23.6 \mathrm{eV}}{e^{-}}$is the work function for ionising an argon atom 107;

- $R=0.62$ is the recombination factor obtained with the Modified Box Model 108 at MicroBooNE electric field of $270 \mathrm{~V} / \mathrm{cm}$ assuming an energy loss per length $d E / d x=2.3 \mathrm{MeV} / \mathrm{cm}$.

The reconstructed energy is obtained by summing the energy of each hit on the collection plane from reconstructed and electron showers with their starting point contained in the fiducial volume. Figure 6.19 shows the calibration slope necessary to convert the electron reconstructed energy $E_{\text {reco }}^{e}$ into true electron deposited energy $E^{e}$. The true energy spectrum has been divided into 10 bins of equal size in the 30-2030 MeV range. Since the reconstructed energy distributions in each true energy bin are asymmetric, the data points are obtained fitting the distributions with a GaussExp function [109, in order to estimate the most probable value (MPV). The GaussExp function consists of an exponential tail stitched to a Gaussian core and it is often used to measure lossy processes such as the energy reconstructed in a calorimeter. The coordinate on the $E^{e}$ axis is given by the mean of the true energy distribution for each bin. The vertical error bars correspond to the full width at 
half maximum (FWHM) of the fitted function. The true energy distribution and the reconstruction energy distribution for every bin are shown in Figure 6.18.

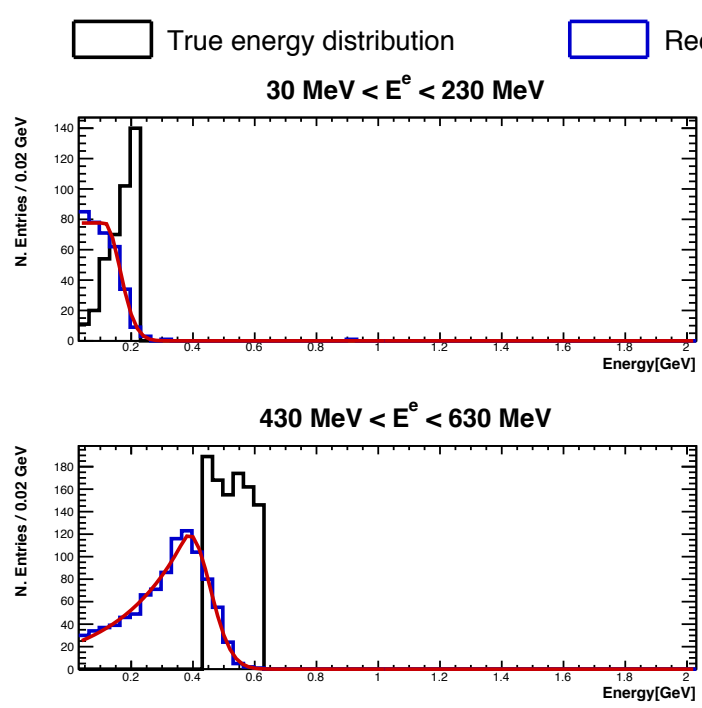

eco. energy distribution

GaussExp fit
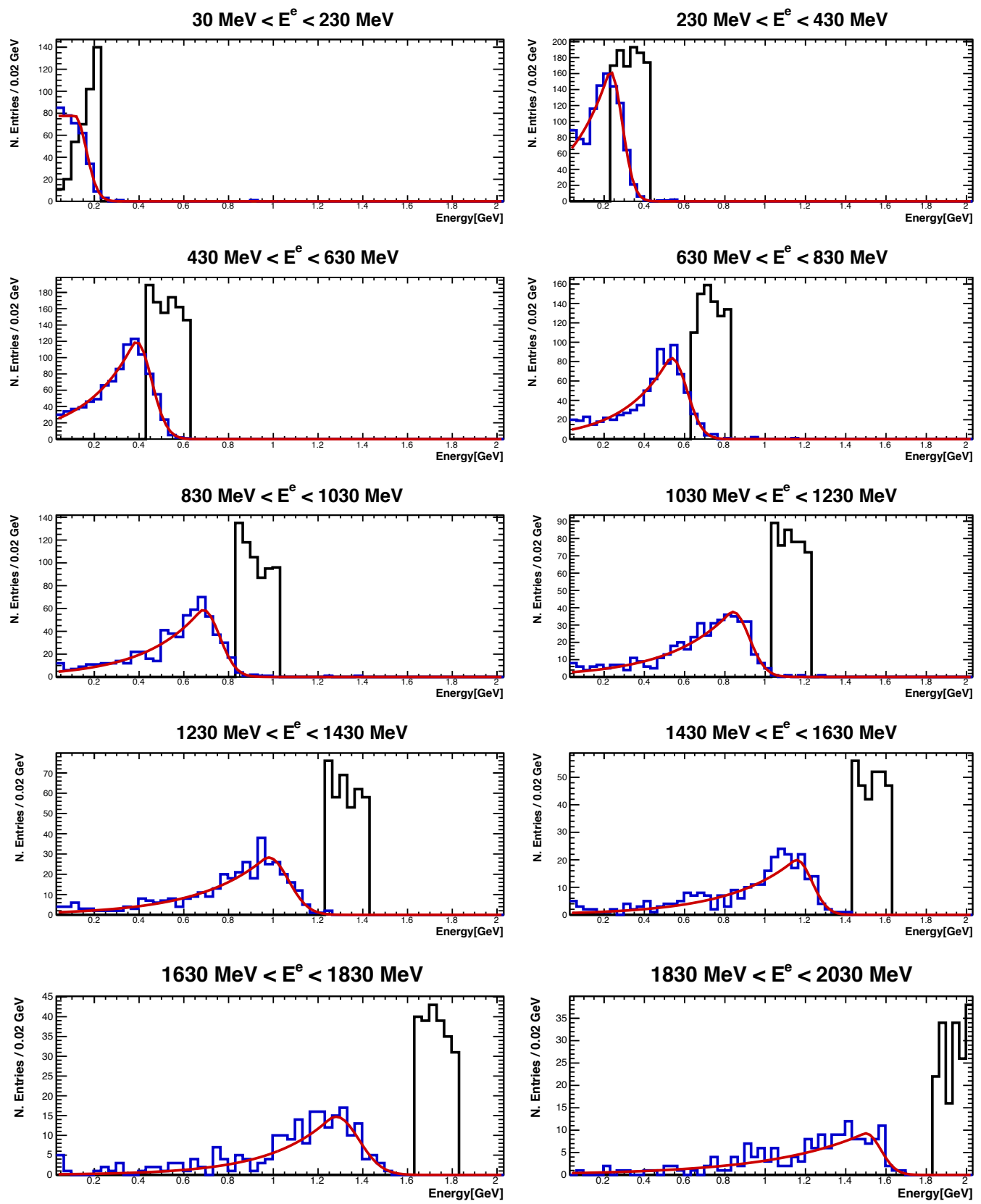

Figure 6.18: Reconstructed (blue) and true (black) energy distribution for 10 intervals of equal size in the 30-2030 MeV energy range. The reconstructed energy distribution have been fitted with a GaussExp function (red line).

The linear fit of the most probable value points, shown in Figure 6.19 gives:

$$
E_{\text {reco }}^{e}=0.77 E^{e}-34.7 \mathrm{MeV} \text {. }
$$


The energy of the shower, corrected by the calibration factor is then defined as:

$$
E_{\text {corr }}^{e}=\left(E_{\text {reco }}^{e}+34.7 \mathrm{MeV}\right) / 0.77 \text {. }
$$

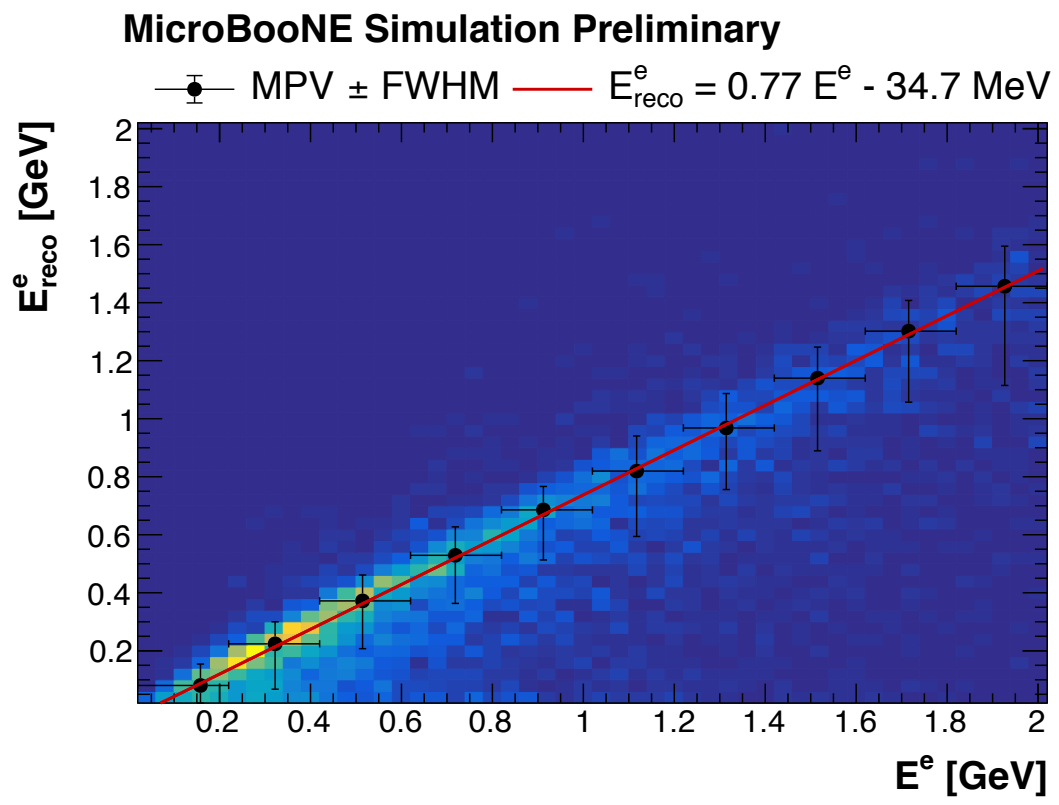

Figure 6.19: Bi-dimensional histogram of reconstructed electron energy $E_{\text {reco }}^{e}$ vs. true electron deposited energy $E^{e}$. The reconstructed electron energy is measured summing the energy of each hit associated to reconstructed showers produced by the simulated electron. The black points correspond to the most probable value of the $E_{\text {reco }}^{e}$ distribution for each $E^{e}$ bin, calculated with a GaussExp fit, as illustrated in Figure 6.18. The red line represents a linear fit of the black points.

It is also possible to measure the energy resolution in the simulation by calculating the normalised difference $E_{\text {frac }}$ between the corrected reconstructed energy $E_{\text {corr }}^{e}$ and the true electron energy $E^{e}$ :

$$
E_{\mathrm{frac}}=\frac{E_{\mathrm{corr}}^{e}-E^{e}}{E^{e}}
$$

Figure 6.20 shows the $E_{\text {frac }}$ distribution for 10 intervals of equal size between 30 and $2030 \mathrm{MeV}$ and the GaussExp fit for each distribution.

The fractional energy resolution can then be defined as the ratio between the standard deviation of the Gaussian core $\sigma$ of the GaussExp function and the true electron energy $E_{e}$. Figure 6.21 shows the fractional energy resolution as a 

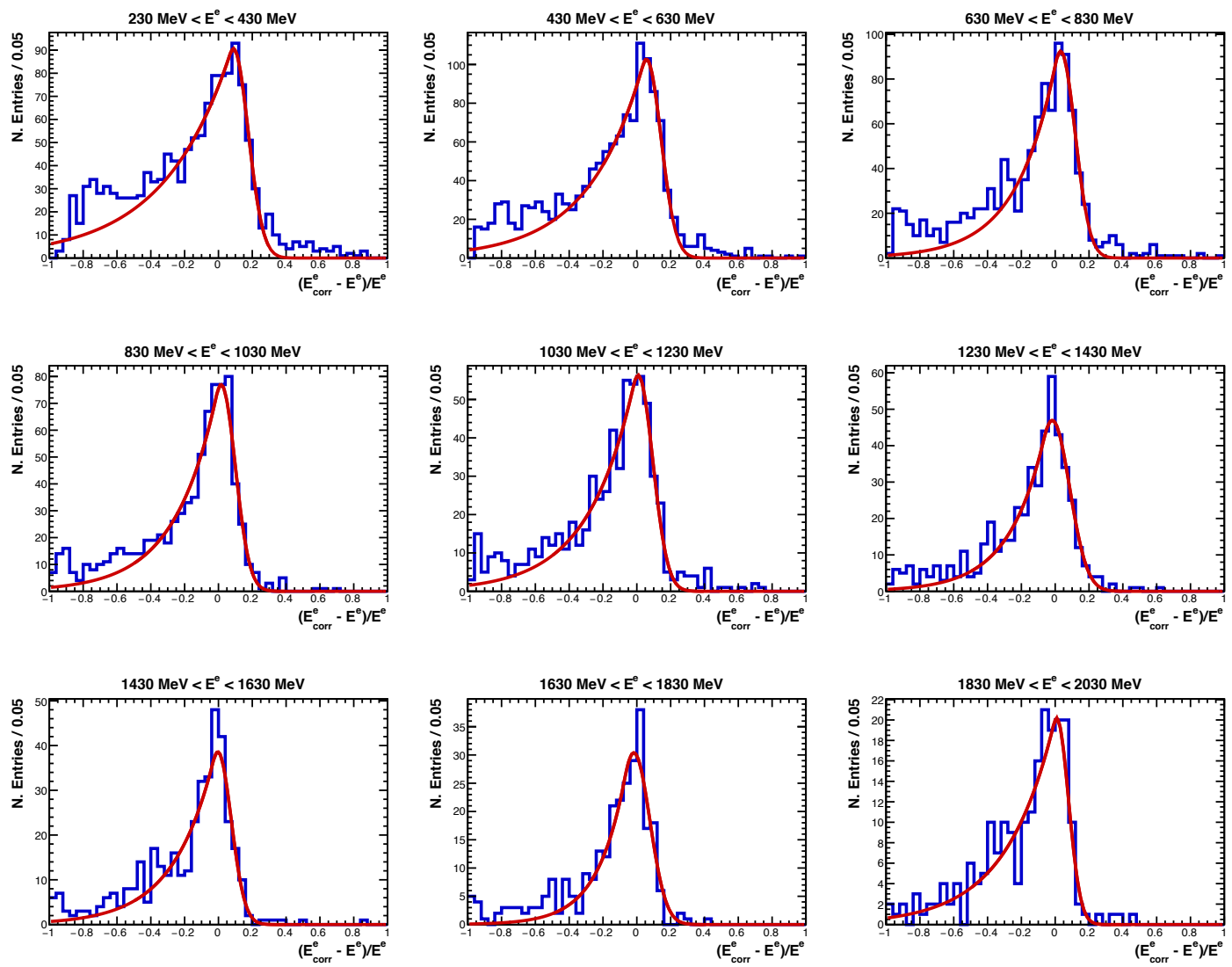

Figure 6.20: Normalised energy difference $E_{\text {frac }}$ for 10 intervals of equal size in the 30-2030 MeV energy range. The normalised energy difference distributions (blue) have been fitted with a GaussExp function (red line).

function of the true electron energy. The points can be fitted with the classic calorimeter resolution formula 110 :

$$
\frac{\sigma}{E}=\frac{a}{\sqrt{E}} \oplus \frac{b}{E} \oplus c
$$

where:

- $a=2.30 \%$ is the stochastic term, which is caused by the intrinsic fluctuations of the development of the electromagnetic shower;

- $b=7.53 \%$ is the noise term, which comes from the electronics noise of the readout chain and represents the dominant contribution;

- $c=1.21 \%$ is the constant term, which is caused by detector non-uniformities (e.g. the presence of unresponsive wires). 


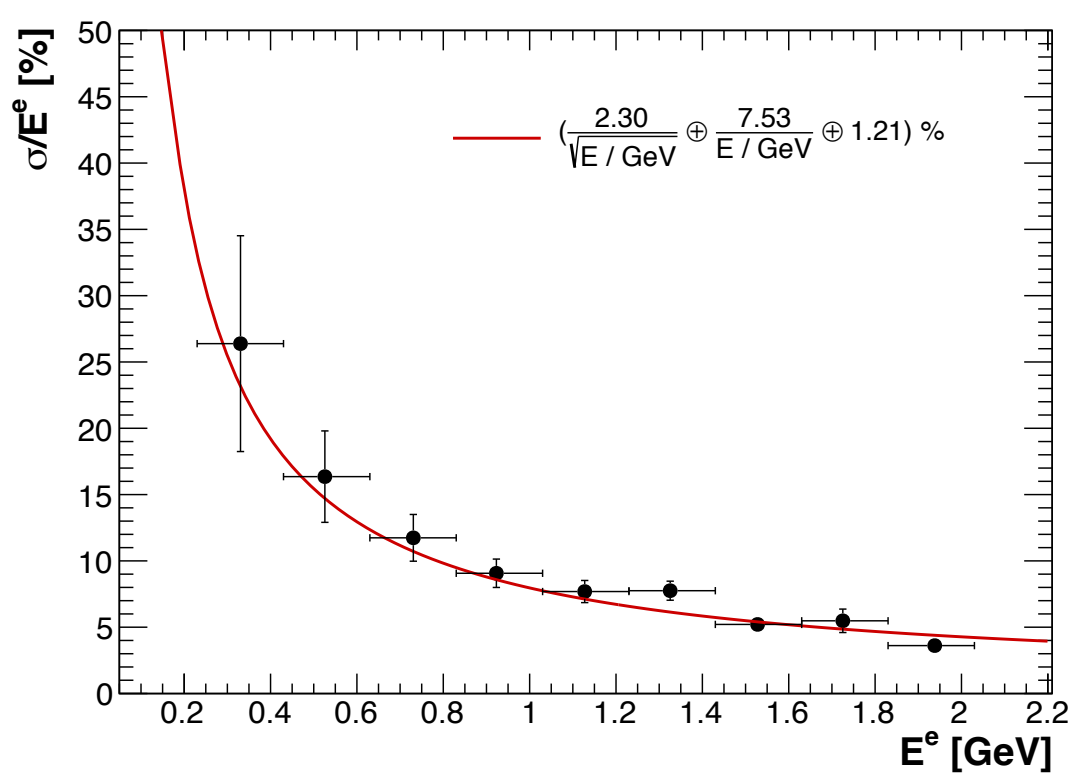

Figure 6.21: Fractional energy resolution for 10 intervals of equal size in the 30-2030 $\mathrm{MeV}$ energy range. The points, extracted from simulated events, have been fitted the classic calorimeter energy resolution equation 6.9 (red line).

Another effect which contributes to the broadening of the energy resolution in MicroBooNE is caused by wrong or sub-optimal clustering: if the pattern recognition fails to group together all the hits that correspond to the electromagnetic shower, or if it includes hits belonging to ionisation tracks (e.g. from cosmic muons), the reconstructed energy will be respectively smaller or larger than the true electron energy. It is also important to underline that the energy resolution quoted here does not correspond to the intrinsic MicroBooNE energy resolution, but only to the energy resolution for the selected $\nu_{e} \mathrm{CC} 0 \pi$-Np events.

\subsubsection{Single proton energy reconstruction and calibration}

Proton energy reconstruction is performed by converting the reconstructed track length $L$ into deposited energy using the proton stopping power in liquid argon tabulated in 111. Liquid argon density $\rho_{\mathrm{LAr}}$ is assumed to be constant at $1.4 \mathrm{~g} / \mathrm{ml}$. Figure 6.22 shows the proton kinetic energy as a function of the range of the proton in liquid argon (measured as $\left.L \times \rho_{\mathrm{LAr}}\right)$. 


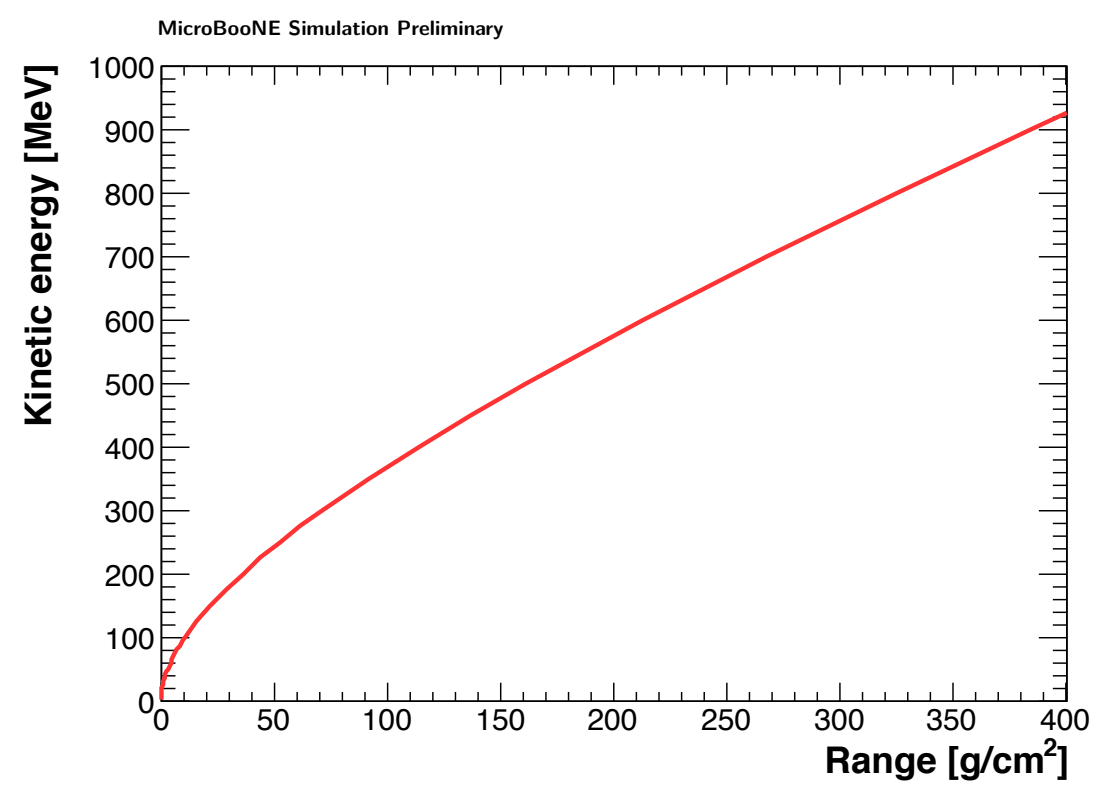

Figure 6.22: Proton kinetic energy as a function of the range of the proton in liquid argon, as tabulated in 111.

The calibration constant has been obtained comparing the reconstructed energy of the proton with the true kinetic energy of the simulated proton, in a $\mathrm{CC} \nu_{e}$ sample with only one proton in the final state. The true proton and the reconstructed tracks are required to be fully contained within the fiducial volume. Since protons are not minimum-ionising particles, in the case of two or more tracks (split tracks) associated to the same proton, the reconstructed length of the tracks has been summed before calculating the corresponding kinetic energy. Figure 6.23 shows the calibration slope necessary to convert the proton reconstructed energy $E_{\text {reco }}^{p}$ into true proton kinetic energy $E^{p}$. For each bin of the true proton energy, the most probable value of the corresponding proton reconstructed energy has been obtained with a GaussExp fit. A linear fit of the most probable values gives:

$$
E_{\mathrm{reco}}^{p}=1.00 E^{p}-2.9 \mathrm{MeV}
$$

The energy of the track, corrected by the calibration factor is then defined as:

$$
E_{\text {corr }}^{p}=\left(E_{\text {reco }}^{p}+2.9 \mathrm{MeV}\right) / 1.00 \text {. }
$$




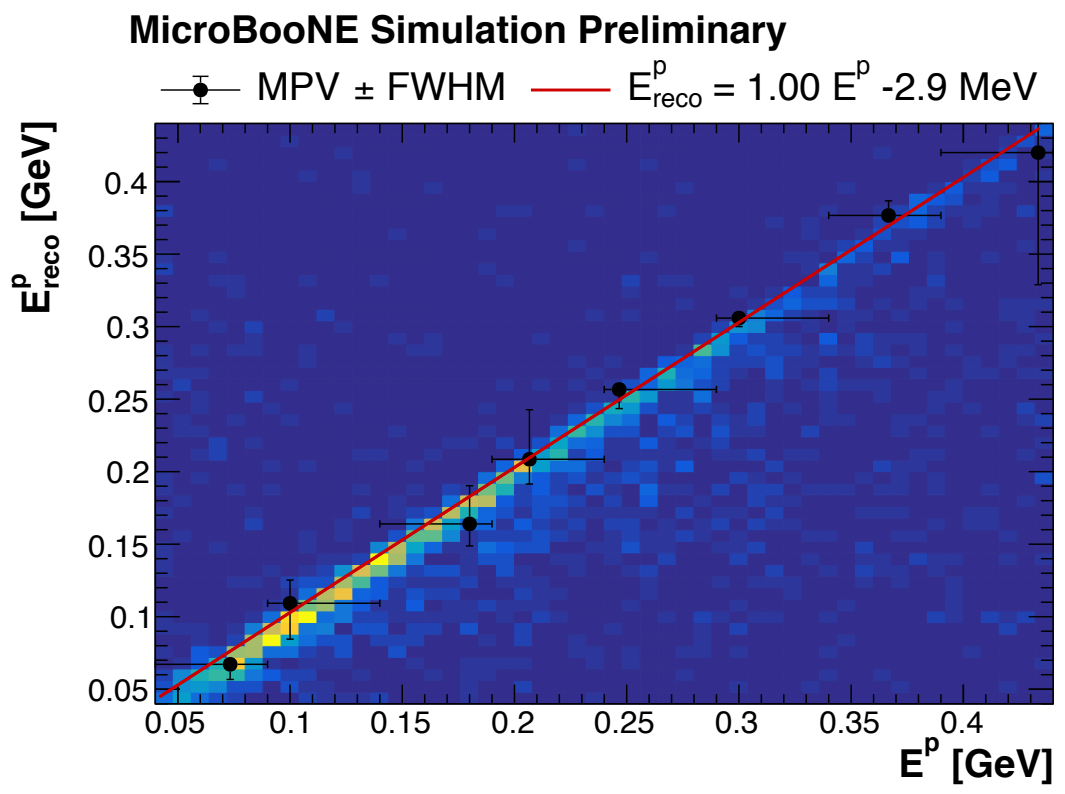

Figure 6.23: Bi-dimensional histogram of true proton energy $E^{p}$ vs. reconstructed proton energy $E_{\text {reco }}^{p}$. The calibration is calculated from a linear fit of the most probable values of the $E_{\text {reco }}^{p}$ distribution for each $E^{p}$ bin. The black points correspond to the most probable value of the $E_{\text {reco }}^{p}$ distribution for each $E^{p}$ bin, calculated with a GaussExp fit. The red line represents a linear fit of the black points.

\subsubsection{Deposited Energy Reconstruction}

It is possible to compare the total visible energy in the event $E_{\mathrm{k}}$, defined as the sum of the kinetic energies of the visible particles in the final state, with the sum of the reconstructed energies for shower-like $\left(E_{\text {corr }}^{e}\right)$ and track-like objects $\left(E_{\text {corr }}^{p}\right)$ for the selected $\nu_{e} \mathrm{CC} 0 \pi-\mathrm{Np}$ events. This quantity $E_{\text {corr }}$ is defined as:

$$
E_{\mathrm{corr}}=\sum^{N_{p}} E_{\mathrm{corr}}^{p}+\sum^{N_{e}} E_{\mathrm{corr}}^{e}
$$

where $N_{p}$ is the number of reconstructed tracks and $N_{e}$ is the number of reconstructed showers in the event. For events where we have two or more shower-like objects and no track-like objects, the shower-like object with the lowest proton $\chi^{2}$ score is chosen as proton candidate (see Section 6.3.7). In these cases we have $N_{p}=1$ by definition. The reconstructed energy does not include particles that do not interact in the liquid argon (such as neutrons) and charged particles with a kinetic energy below the detection threshold, defined in Section 6.2.7. Figure 6.24 shows the calibration slope necessary to convert the total reconstructed energy $E_{\text {corr }}$ into 
visible energy $E_{\mathrm{k}}$. The plot has been obtained using the $\nu_{e} \mathrm{CC} 0 \pi-\mathrm{Np}+\operatorname{cosmic}$ simulation sample. A linear fit of the data points gives:

$$
E_{\mathrm{k}}=0.98 E_{\text {corr }}-28.5 \mathrm{MeV} .
$$

The reconstructed visible energy, corrected by the calibration factor is then defined as:

$$
E_{\text {deposited }}=\left(E_{\text {corr }}+28.5 \mathrm{MeV}\right) / 0.98 \text {. }
$$

Several effects can contribute to this calibration factor: among others, the presence of regions with unresponsive wires can cause an underestimation of the deposited energy. In the future, this effect can be limited by the use of the other two planes for calorimetric measurements.

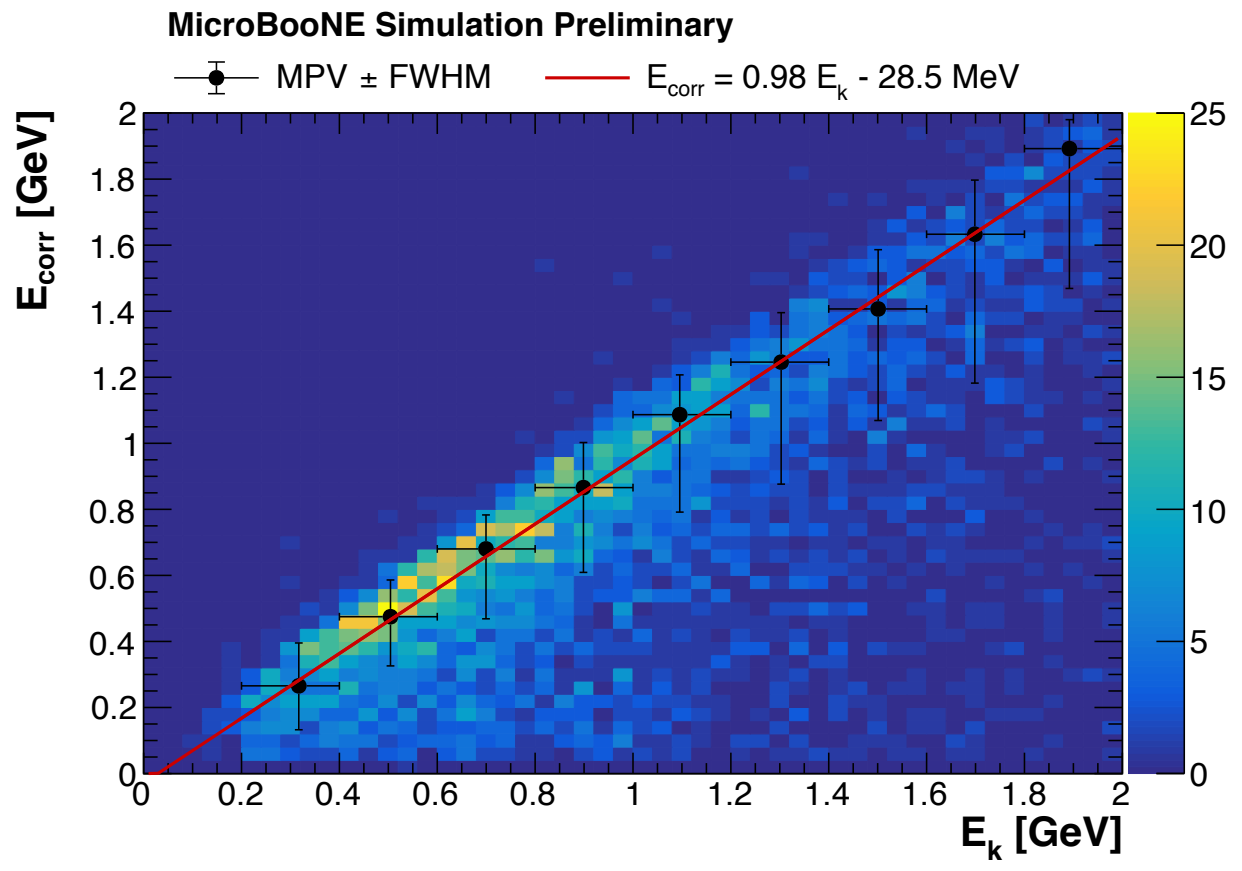

Figure 6.24: Bi-dimensional histogram of the total reconstructed energy $E_{\text {corr }}$ vs. the total visible energy $E^{\mathrm{k}}$ vs. Black points are obtained measuring the most probable value of the $E_{\text {corr }}$ distribution for each $E_{\mathrm{k}}$ bin, obtained with a GaussExp fit.

In this analysis, we will use the quantity $E_{\text {deposited }}$ defined in Equation 6.14 as estimate of the total visible energy in the event. 


\subsubsection{Deposited energy binning}

The binning of the deposited energy distribution must be carefully evaluated, since it is of fundamental importance in the calculation of the significance of an eventual excess. In this analysis, we require the events in a true $E_{\mathrm{k}}$ bin to fall in the same reconstructed $E_{\text {deposited }}$ bin in at least $50 \%$ of the cases for $\nu_{e} \mathrm{CC} 0 \pi-\mathrm{Np}$ events. A combination which satisfies this condition and maximises the number of bins in the $[0,3] \mathrm{GeV}$ range is:

$$
E_{\text {deposited }} \text { bins }=[0,0.2,0.4,0.6,0.9,1.25,1.9,3] \mathrm{GeV},
$$

which is then chosen as our binning for the $E_{\text {deposited }}$ distributions. Figure 6.25 shows the migration matrix between $E_{\text {deposited }}$ and $E_{\mathrm{k}}$. It shows the probability that an event with a true energy $E_{\mathrm{k}}$ in the $i$ bin has a reconstructed energy $E_{\text {deposited }}$ in the $j$ bin. Our binning criteria ensures that each element on the diagonal has a value larger than 0.50 .

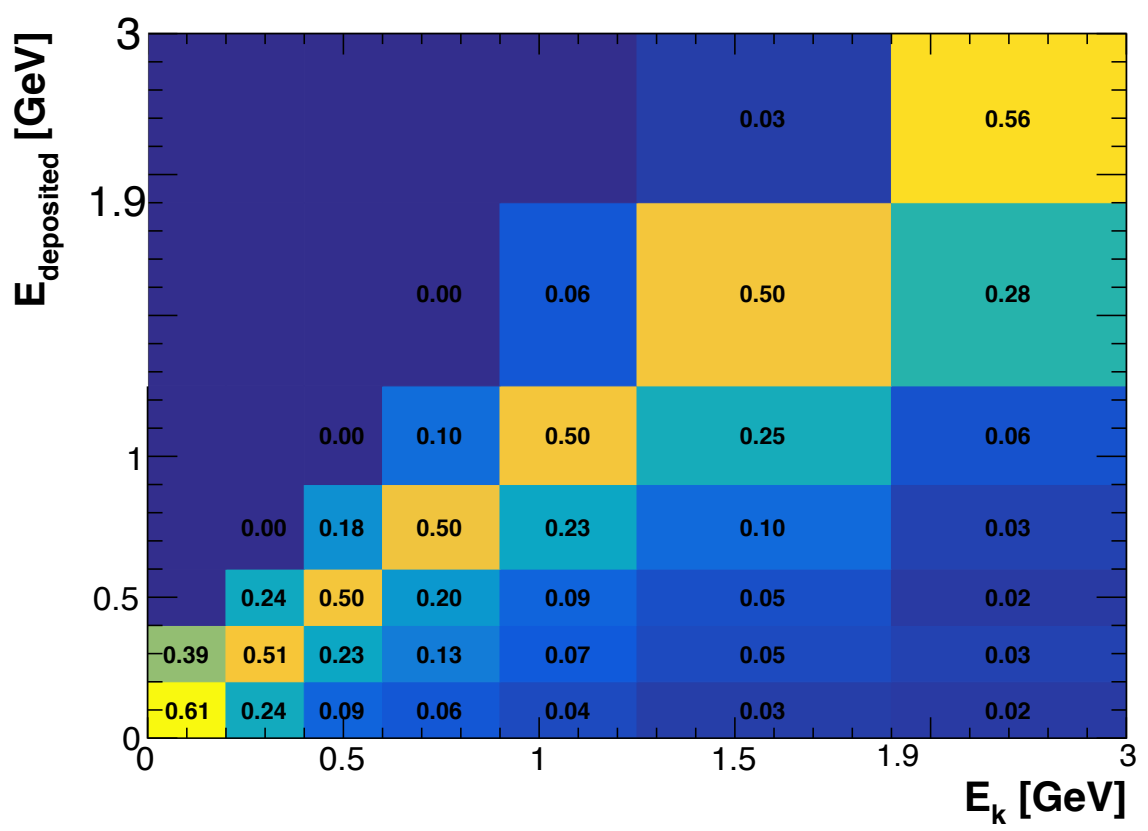

Figure 6.25: Migration matrix between $E_{\text {deposited }}$ and $E_{\mathrm{k}}$. It shows the probability that an event with a true energy $E_{\mathrm{k}}$ in the $i$ bin has a reconstructed energy $E_{\text {deposited }}$ in the $j$ bin. 


\subsubsection{Measurement of the electromagnetic shower energy loss}

The mean energy loss per length of charged particles $\langle d E / d x\rangle$ can be described by the Bethe equation as defined in $[19]$ :

$$
-\left\langle\frac{d E}{d x}\right\rangle=K z^{2} \frac{Z}{A} \frac{1}{\beta^{2}}\left[\frac{1}{2} \ln \frac{2 m_{e} c^{2} \beta^{2} \gamma^{2} T_{\max }}{I^{2}}-\beta^{2}-\frac{\delta(\beta \gamma)}{2}\right],
$$

where $T_{\max }$ is the maximum possible energy transfer in a single collision, $I$ is the mean excitation energy, and $\delta(\beta \gamma)$ is a density correction.

In materials of moderate thickness such as LAr, the energy loss probability distribution is described by the asymmetric Landau distribution [112, which drives the mean of the energy loss of Equation 6.16 into the tail of the distribution. For this reason, "the mean of the energy loss given by the Bethe equation [...] is thus ill-defined experimentally and is not useful for describing energy loss by single particles" 19. The most probable value of the Landau distribution, which should be used instead, is given by:

$$
\Delta_{p}=\xi\left[\ln \frac{2 m c^{2} \beta^{2} \gamma^{2}}{I}+\ln \frac{\xi}{I}+j-\beta^{2}-\delta(\beta \gamma)\right],
$$

where $\xi=(K / 2)\langle Z / A\rangle\left(x / b^{2}\right) \mathrm{MeV}, j=0.2$, and $x$ is the thickness of the material in $\mathrm{g} \cdot \mathrm{cm}^{2} 19$.

An important feature of the LArTPC technology is its ability to distinguish between electrons and photons in the final state by measuring their $d E / d x$. Photons that undergo pair-production, which is the dominant process above $10 \mathrm{MeV}$, produce a $e^{+} e^{-}$pair. If the pair is boosted, the trajectories of the positron and the electron overlap, producing an average $d E / d x$ which is twice the one of a single, minimum-ionising electron.

In MicroBooNE, the $d E / d x$ for electromagnetic showers is measured with a procedure analogous to the one developed by the ArgoNeuT collaboration and described in 67 .

In our electromagnetic shower study, as a first step, all the hits on the collection plane within a rectangle of $4 \mathrm{~cm}$ along the direction of the shower and $1 \mathrm{~cm}$ perpendicular to the shower are collected, as shown in the event display in Figure 6.26. 


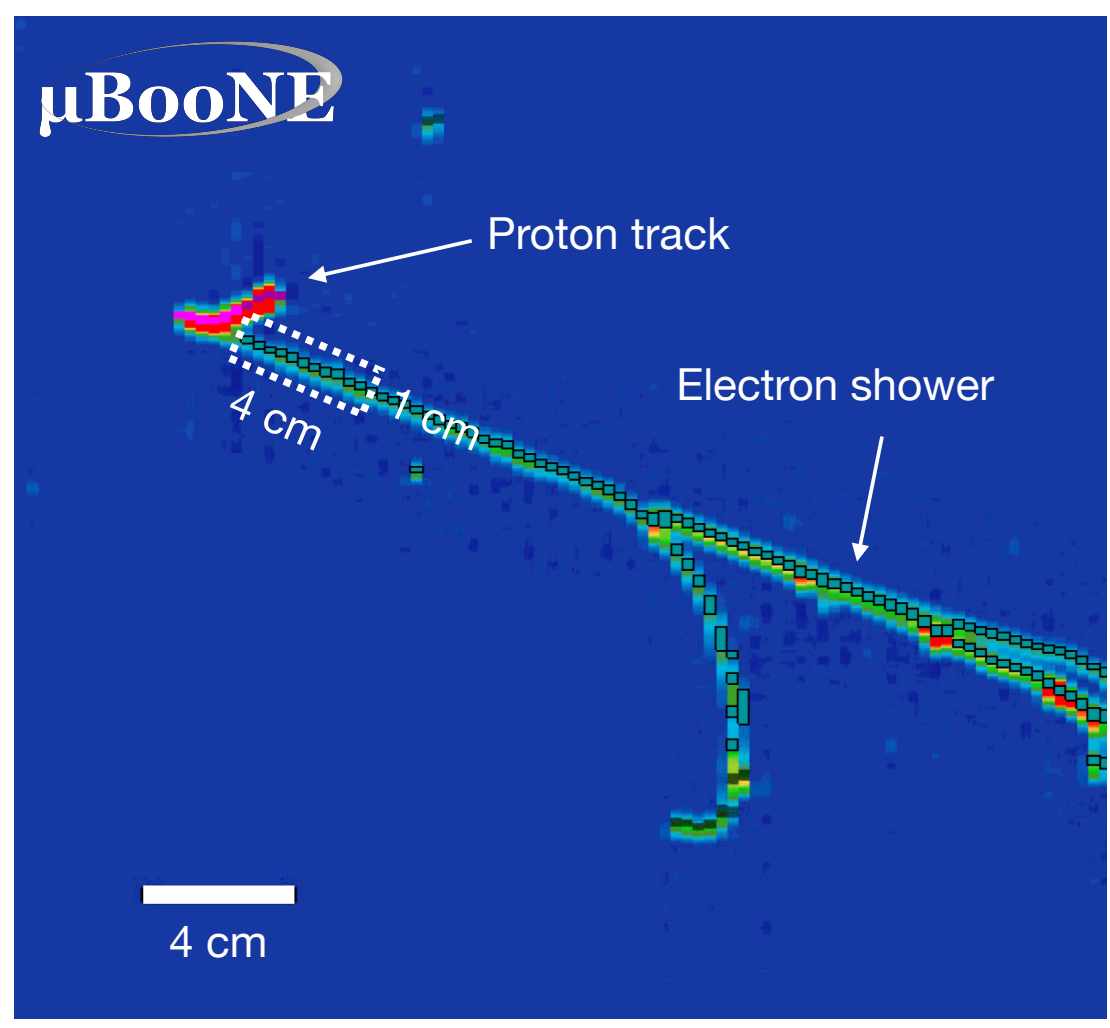

Figure 6.26: Event display of an electron shower candidate, showing the $1 \times 4 \mathrm{~cm}^{2}$ area used for the $d E / d x$ calculation. Each small black rectangle corresponds to a reconstructed shower hit.

Subsequently, the $d Q / d x$ for each hit is measured dividing the collected charge $(d Q)$ by the pitch $(d x)$ between each hit and the next one along the shower direction. The pitch corresponds to the distance in the TPC that a particle travels between its two projections on adjacent wires, which is at least the wire spacing $(3 \mathrm{~mm}$ for MicroBooNE [90|). Electromagnetic showers aligned with the wire direction correspond to a much larger value of the pitch.

The $d E / d x$ is calculated from the $d Q / d x$ using the calibration factor measured in Section 6.3.2, Equation 6.5. Since the Landau distribution of the $d E / d x$ hit values has an asymmetric tail, we assign to the shower the median (and not the mean) of the $d E / d x$ hit distribution, as an estimation of the most probable value. The median metric has been demonstrated in 67 to be the most robust over a variety of box lengths.

Figure 6.27 shows the area-normalised histograms of the median $d E / d x$ in the collection plane for simulated electron and photon showers. The events below 


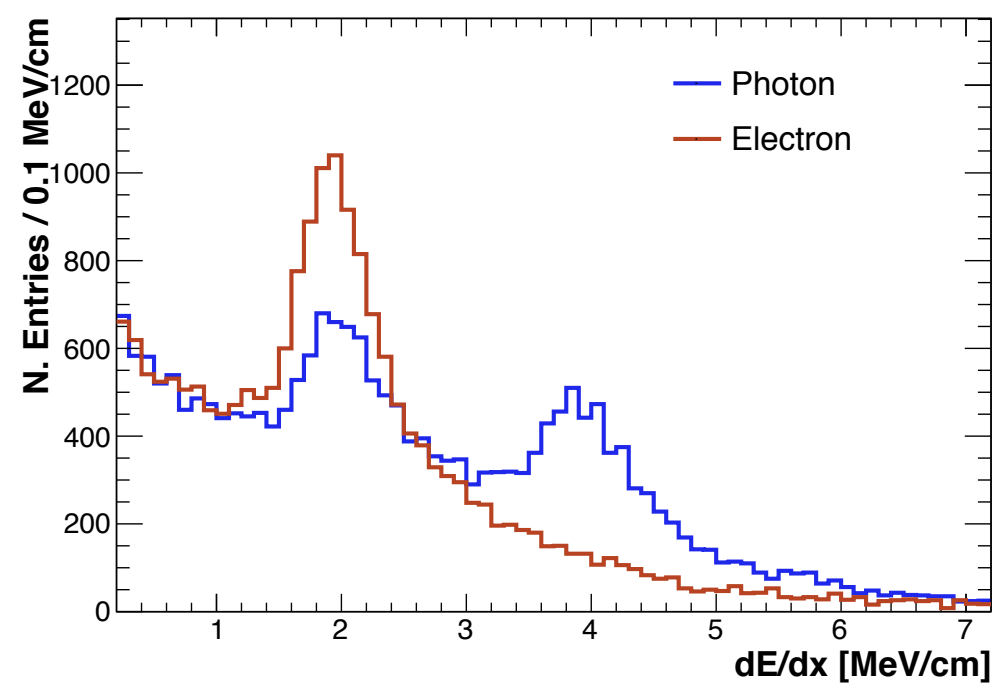

Figure 6.27: Area-normalised distributions of the $d E / d x$ median for simulated electrons (red) and photons (blue) produced in neutrino interactions.

$1 \mathrm{MeV} / \mathrm{cm}$ correspond for both distributions to showers with a low number of associated hits, or where the shower was mostly aligned with the wires of the collection plane (having as such a high pitch value). Figure 6.28 shows the pitch distribution in the collection plane and a bi-dimensional histogram of the $d E / d x$ vs. the pitch for photon showers: events with a $d E / d x$ below $1 \mathrm{MeV} / \mathrm{cm}$ mostly correspond to a high value of the pitch.

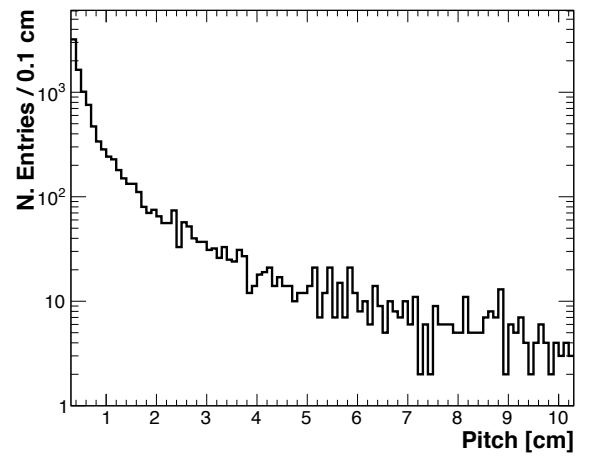

(a) Pitch distribution for simulated electron showers in the collection plane (log-scale).

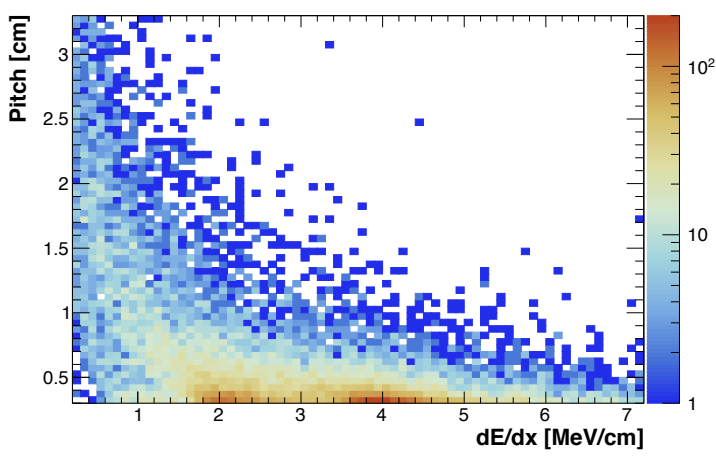

(b) Bi-dimensional histogram of the pitch vs. the measured $d E / d x$ photon showers.

Figure 6.28: Electromagnetic showers aligned with the wire orientation will have a large pitch value and their measured $d E / d x$ will be shifted towards low values.

The electron showers have a peak around $2 \mathrm{MeV} / \mathrm{cm}$ as this is the value for MIPs 
in LAr. The photon showers, instead, have a peak around $4 \mathrm{MeV} / \mathrm{cm}$ (as expected from the $e^{+} e^{-}$pairs) and a second peak around $2 \mathrm{MeV} / \mathrm{cm}$. The $2 \mathrm{MeV} / \mathrm{cm}$ peak for photons is caused by mainly two effects:

- photons that undergo Compton scattering will transfer most of their energy to the electron, producing a shower with the same $d E / d x$ of an electron. This effect decreases with the photon energy and is dominant below $10 \mathrm{MeV}$, as shown in Figure 6.29 .

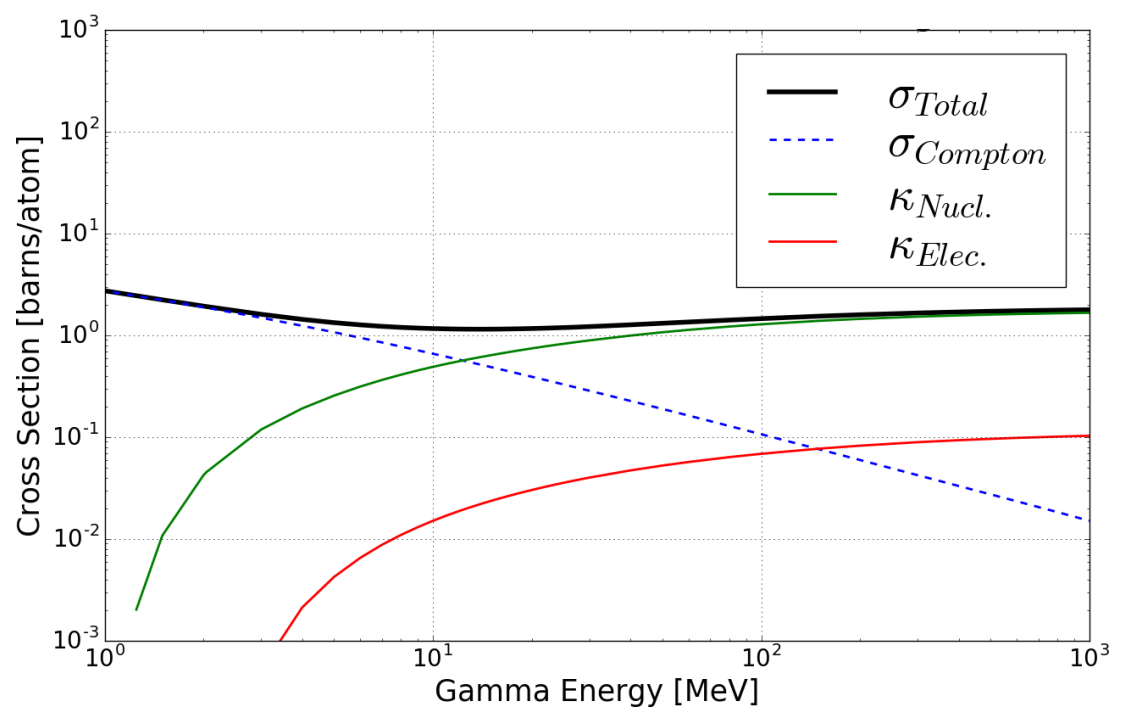

Figure 6.29: Cross section of gammas on argon between $1 \mathrm{MeV}$ and $1 \mathrm{GeV}$. Here, $\kappa$ refers to the pair production cross section for the nuclear field and electron field. Compton scattering is dominant below $10 \mathrm{MeV}$. From 67 .

- very asymmetric $\gamma \rightarrow e^{+} e^{-}$pair production. When the positron and the electron do not overlap, the measured $d E / d x$ distribution will be peaked around $2 \mathrm{MeV} / \mathrm{cm}$. The electron and the positron can also be produced with very different energies, as shown in Figure 6.30. In this case, the distribution of the $d E / d x$ values in the $1 \times 4 \mathrm{~cm}^{2}$ box will be shifted towards $2 \mathrm{MeV} / \mathrm{cm}$.

Figure 6.27 represents the limitation to the $e / \gamma$ separation capabilities in the current version of the analysis A comparison between the data and Monte Carlo distributions of the reconstructed showers $d E / d x$ is shown in Figure 6.37. 


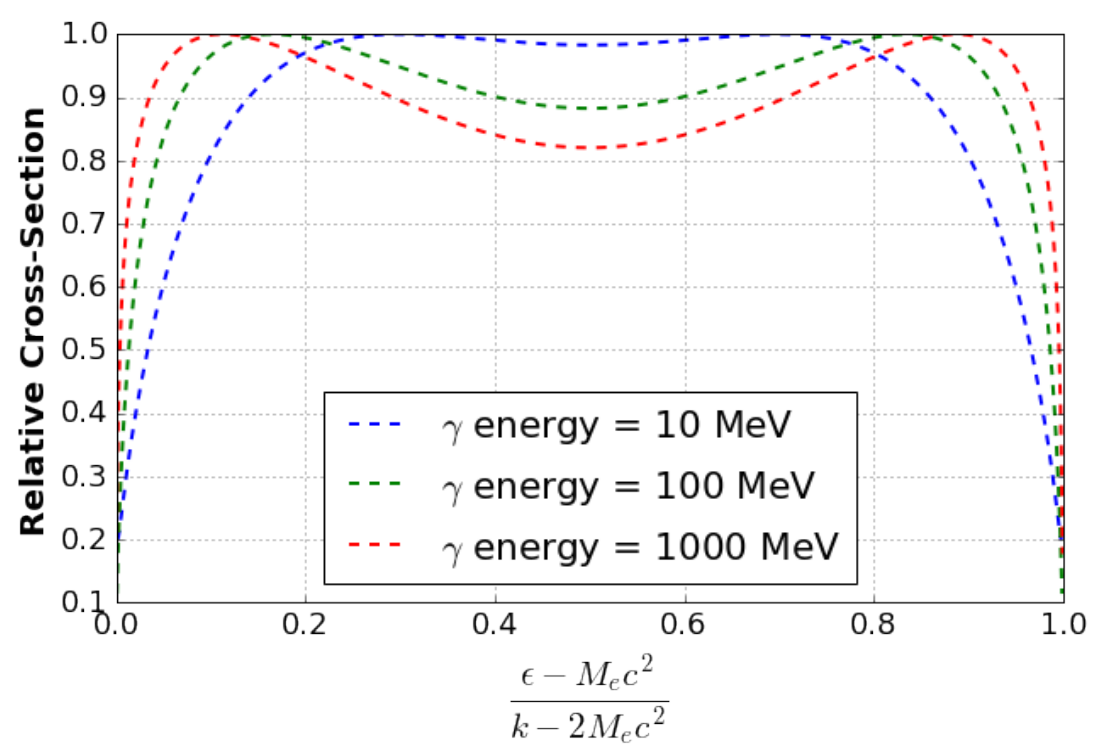

Figure 6.30: Pair production relative cross section as a function of the fraction of the photon energy $k$ transferred to either the electron or positron with energy $\epsilon$ for different photon energies. From 113.

\subsubsection{Particle identification of reconstructed tracks}

The measurement of the $d E / d x$ along a reconstructed track allows to perform powerful particle identification, as demonstrated by a the ArgoNeuT collaboration in 108 .

This capability is particularly important in the search of low-energy electron neutrinos. A $\nu_{e} \mathrm{CC} 0 \pi-1 \mathrm{p}$ neutrino interaction is topologically identical to a cosmic muon stopping in the LAr, since they both appear as a track with a small shower attached. However, the energy loss of a proton produced in a neutrino interaction is sensibly different from the one of a cosmic muon, for two main reasons:

- the Bragg peak of the stopping cosmic muon corresponds to the starting point of the Michel electron shower, while the Bragg peak of the proton track is far from the interaction vertex;

- the proton $d E / d x$ profile for protons differs from the $d E / d x$ profile for muons.

Being able to distinguish between proton and muon tracks is therefore essential in order to reject cosmogenic background. Events with a $\nu_{\mu}$ interaction with only a stopping muon in the final state can also be rejected with the same technique. The stopping power $d E / d x$ can be used to determine the range of the particle, by 
integrating the deposition until the particle is stationary, as derived in $[114$. It is then possible to parametrise the relation between the theoretical $d E / d x$ of a ionisation track and its residual range with a power-law function:

$$
\frac{d E}{d x}=A R^{b}
$$

where $A$, which has dimensions $\mathrm{MeV} / \mathrm{cm}^{1-b}$, and $b$, which is dimensionless, depend on the particle which produced the ionisation trail. The residual range $R$, in this case measured in centimetres, is the distance between a point on the track and its end ( $R=0$ correspond to the track end-point). Figure 6.31 shows the $d E / d x$ as a function of the residual range $R$ for several particle types. The values of $A$ and $b$ for each particle type are reported in Table 6.2 .

Table 6.2: Stopping power parametrisation for various particle types in liquid argon (from 108]).

\begin{tabular}{lcr}
\hline Particle type & $A\left[\mathrm{MeV} / \mathrm{cm}^{1-b}\right]$ & $b$ \\
\hline Proton & 17 & -0.42 \\
Kaon & 14 & -0.41 \\
Pion & 8 & -0.37 \\
Muon & 7 & -0.36 \\
\hline
\end{tabular}

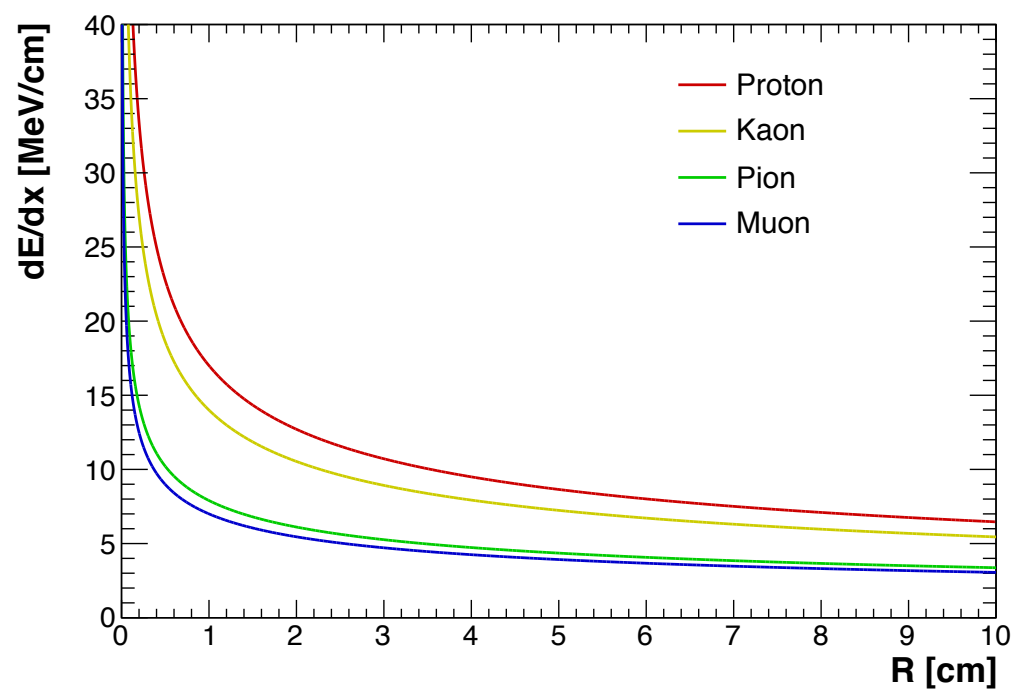

Figure 6.31: Parametrised $d E / d x$ in liquid argon for various particles as a function of the residual range $R[108$. Parameters values can be found in Table 6.2 
The measured $d E / d x$ vs. $R$ profile can be compared with the theoretical expectation and the score of the $\chi^{2}$ test can be computed for different particle hypothesis. Figure 6.32 shows the $\chi^{2}$ score in the proton hypothesis for reconstructed proton tracks and reconstructed muon tracks in a Monte Carlo simulation. As expected, proton tracks are peaked at low values of the $\chi^{2}$, while the muon tracks correspond to much larger $\chi^{2}$ scores.

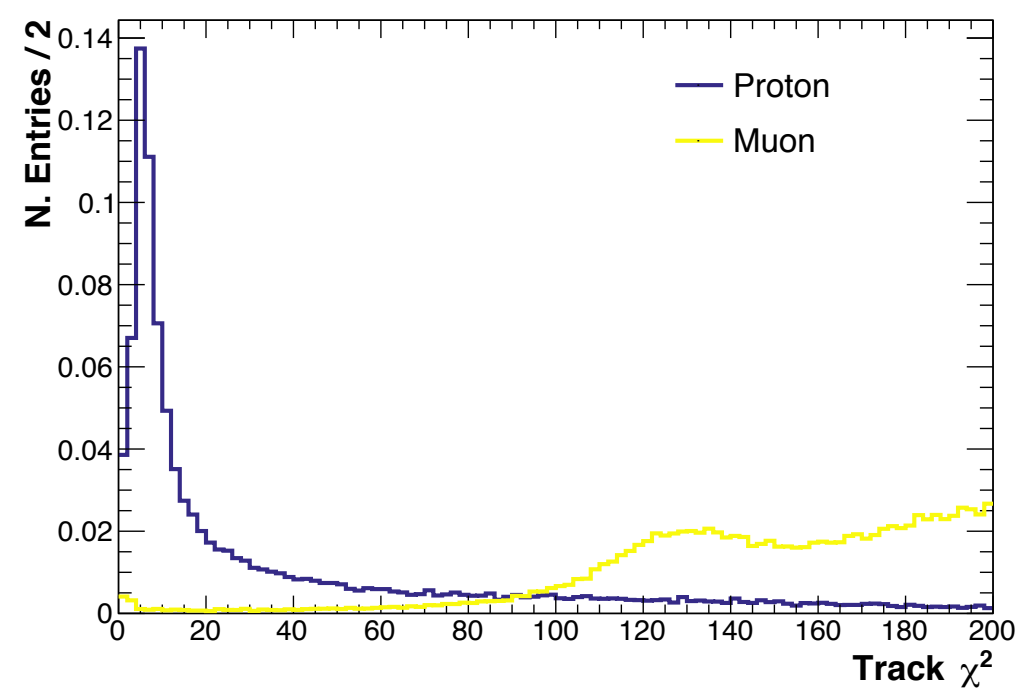

Figure 6.32: Area-normalised distributions for the $\chi^{2}$ score in the proton hypothesis for reconstructed proton tracks (blue) and reconstructed muon tracks (yellow) in Monte Carlo simulations.

A comparison between the data and Monte Carlo distributions of the $\chi^{2}$ score in the proton hypothesis is shown in Figure 6.40.

\section{Selected event spectrum}

Figure 6.33 shows the reconstructed deposited energy spectrum of the selected events. The reconstructed deposited energy $E_{\text {deposited }}$ has been measured with the procedure described in Section 6.3.5. Both the $\chi^{2}$ and Kolmogorov-Smirnov 115 tests give a probability of 1.00 (rounded up at the second decimal digit) of the null hypothesis, which is the data distribution being compatible with the Monte Carlo simulation. 


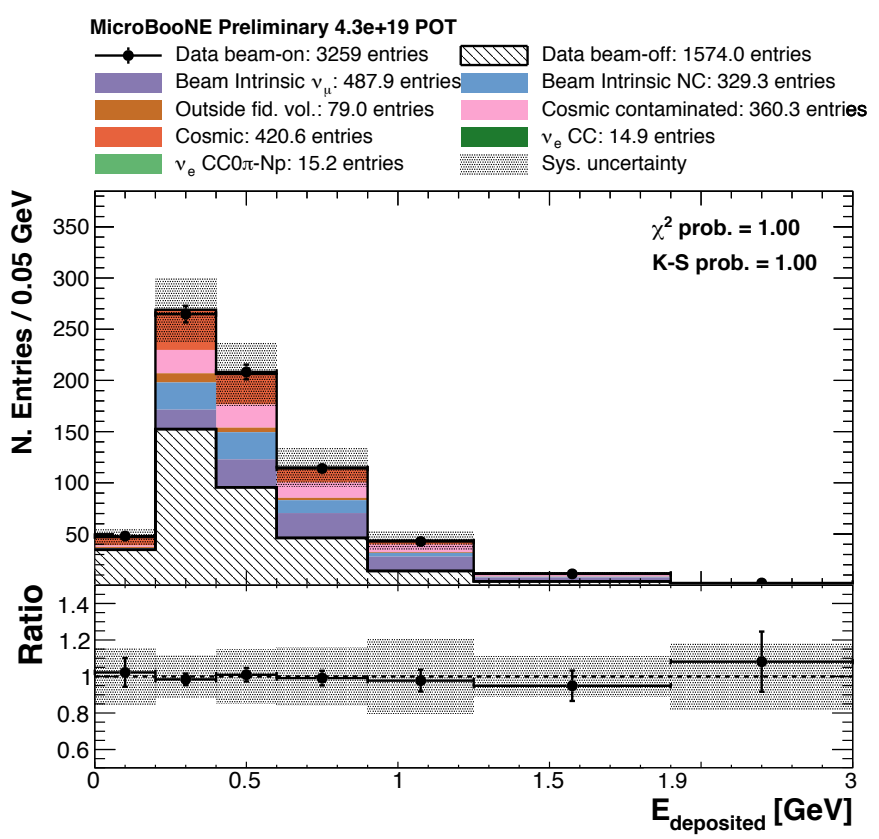

Figure 6.33: Reconstructed energy spectrum after the event selection algorithm and the veto of the events selected by the $\mathrm{CC} \nu_{\mu}$ module. The histograms of the event categories are stacked.

\subsection{Background Rejection}

In this section we will describe the cuts we can apply to our selected events in order to isolate the $\nu_{e} \mathrm{CC} 0 \pi$-Np event candidates. The cuts have been chosen to (1) reduce the background, and (2) ensure that the selected events are well reconstructed. The values of each cut have been chosen manually to maximise the $\nu_{e} \mathrm{CC} 0 \pi$-Np purity while retaining a sufficient efficiency. These cuts could be further optimised by using a larger data sample, which the collaboration will make available in the near future.

\subsubsection{Rectangular cuts}

The goal of the rectangular cuts is to isolate the $\nu_{e} \mathrm{CC} 0 \pi$-Np events and increase the purity of our selected sample. However, in order to validate our cuts and verify the agreement between data and Monte Carlo after this stage, it is necessary to select a sufficient number of data events. In particular, in this analysis we require at least one data event per bin in the $[0.2,1.9] \mathrm{GeV}$ range of the $E_{\text {deposited }}$ spectrum. 
In this section we will study all the variables used to apply the kinematic and calorimetric cuts. In particular, we will show, for each variable:

- the area-normalised Monte Carlo distributions for the signal $\left(\nu_{e} \mathrm{CC} 0 \pi-\mathrm{Np}\right.$ events), the cosmogenic background (cosmic, cosmic contaminated, and cosmic in-time), and the neutrino background $\left(\nu_{e} \mathrm{CC}\right.$, beam intrinsic $\nu_{\mu}$, beam intrinsic $\mathrm{NC}$, and outside fid. vol.), to verify the rejection power of each cut;

- the POT-normalised Monte Carlo and data distributions, to ensure that the collected data agree with the simulation with the collected data. The Monte Carlo events are categorised according to the event type;

- for shower and track variables, the POT-normalised data and Monte Carlo distributions, categorised according to the particle type.

Number of reconstructed hits $>50$

A large number of delta rays and cosmic-ray remnants not tagged by the cosmicray removal algorithm may fake a neutrino candidate with a low number of hits. Thus, we require at least 50 reconstructed hits in the collection plane to reduce this cosmogenic background. The area-normalised distributions in Figure 6.34 a show that a large fraction of the cosmogenic backgrounds has a very low number of reconstructed hits in the collection plane, while the signal and the neutrino components have a much broader distribution. Figure 6.34b shows a good data/Monte Carlo agreement for this variable.

\section{Showers energy $>50 \mathrm{MeV}$}

For the same reason as above, the energy of the showers associated with delta rays or Michel electrons will be peaked at low energies. We require the sum of the energies of the reconstructed showers to be above $50 \mathrm{MeV}$. In this way we reject a large fraction of the neutrino and cosmic background, without significantly affecting the number of $\nu_{e} \mathrm{CC} 0 \pi-\mathrm{Np}$ events, as shown in Figure 6.35a. 


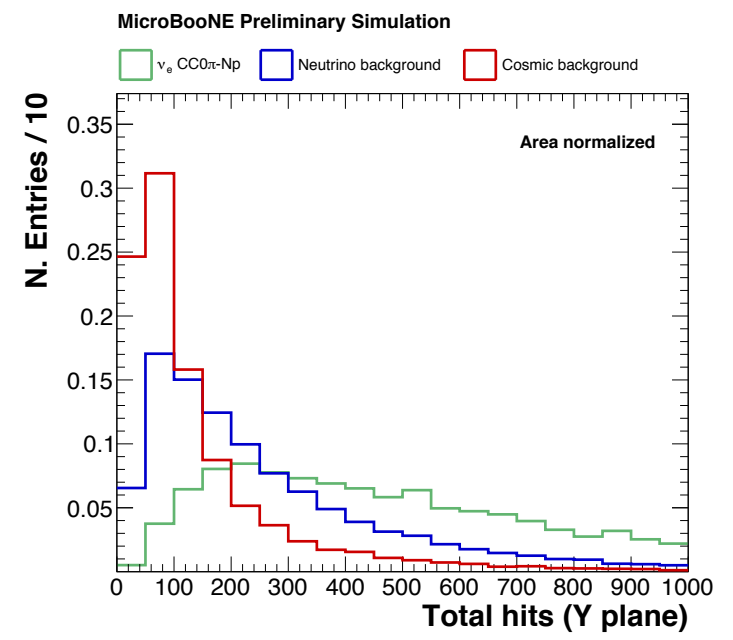

(a) Area-normalised.

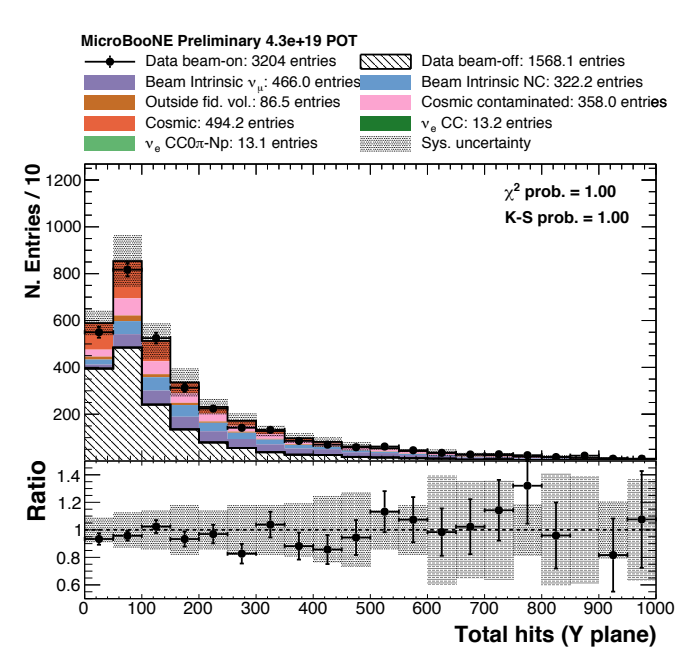

(b) POT-normalised.

Figure 6.34: Area (left) and POT-normalised (right) distributions of the number of reconstructed hits in the collection plane for all the objects in the event. The areanormalised distributions show the $\nu_{e} \mathrm{CC} 0 \pi-\mathrm{Np}$ signal (green), the cosmic background (red), and the neutrino background (blue). The black points in the POT-normalised plot represent the data with statistical uncertainties. The coloured stacked histograms represent the simulated events, with the hatched histogram corresponding to the data beam-off sample. The shaded area represents the systematic uncertainty. The bottom part of the plot shows the ratio between the data beam-on events and the stacked histograms.

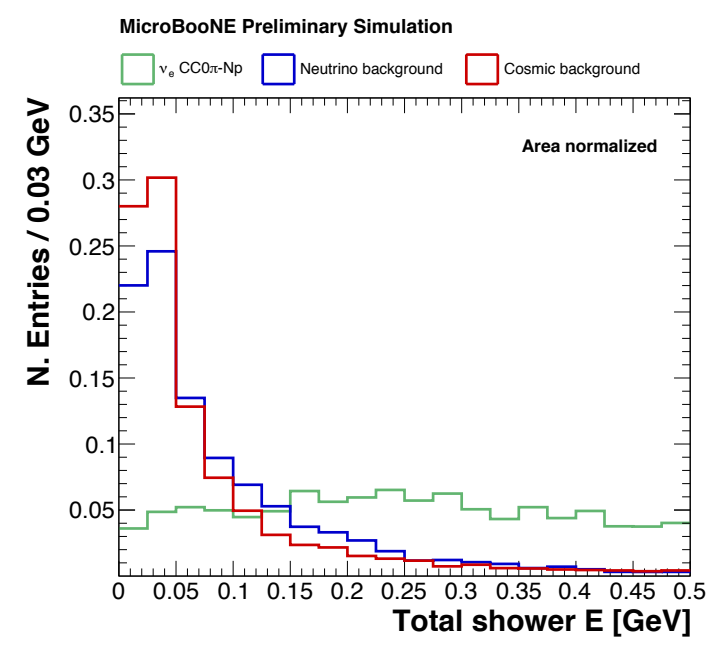

(a) Area-normalised.

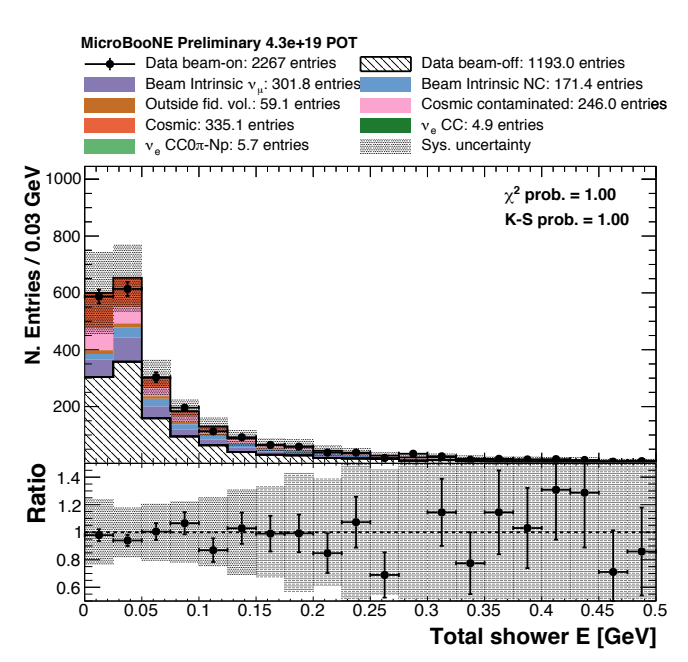

(b) POT-normalised.

Figure 6.35: Area (left) and POT-normalised (right) distributions of the sum of the energies of the reconstructed showers in each event. 


\section{Fraction of shower hits $>0.5$}

Cosmic-ray and $\mathrm{CC} \nu_{\mu}$ events faking a $\nu_{e} \mathrm{CC} 0 \pi-\mathrm{Np}$ candidate will have in general a long muon track and a small Michel electron at the end. In these cases, the hits associated with the reconstructed showers will represent a small fraction of the total number of hits, as shown in Figure 6.36a. Signal events, on the contrary, have a large fraction of hits associated to shower objects. We require the ratio between the number of shower hits and track hits to be larger than 0.5. Figure 6.36b shows the agreement between data and Monte Carlo for this quantity.

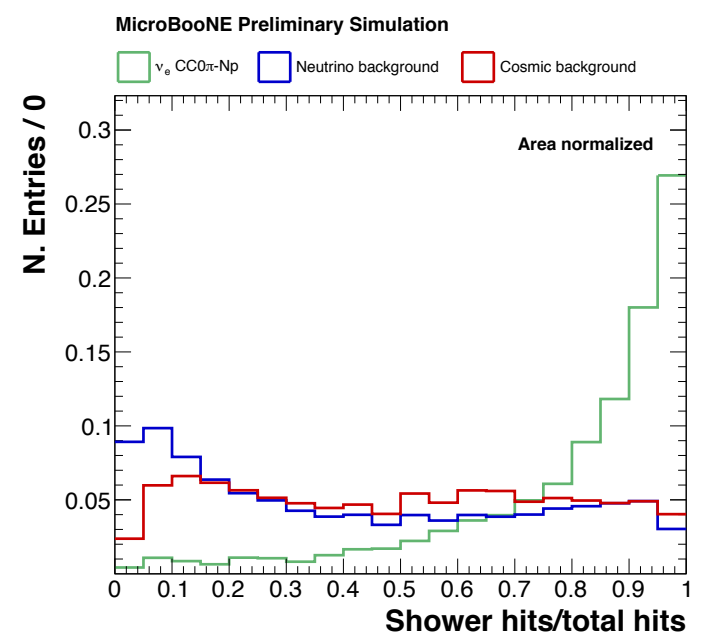

(a) Area-normalised.

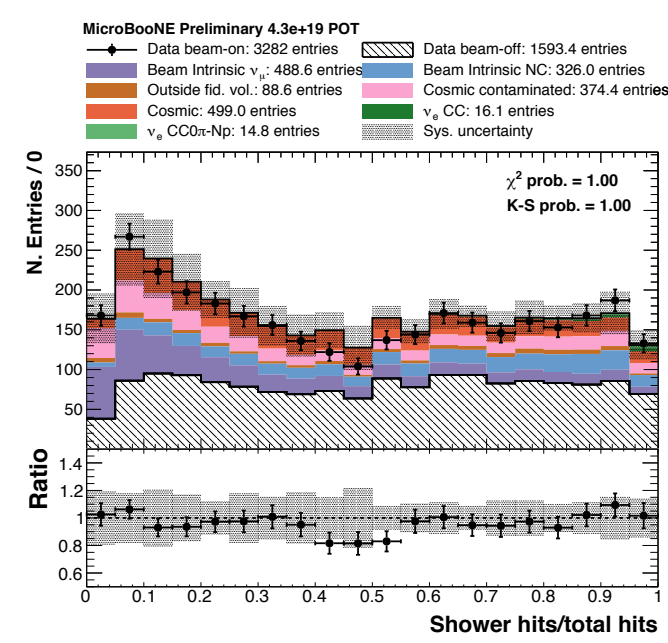

(b) POT-normalised.

Figure 6.36: Area and POT-normalised distributions of the ratio between the hits associated to reconstructed showers and the total number of reconstructed hits in the collection plane.

\section{Most energetic shower $1 \mathrm{MeV} / \mathrm{cm}<d E / d x<3.2 \mathrm{MeV} / \mathrm{cm}$}

Our signal will contain an electron shower, so we require the $d E / d x$ of the leading shower to be around the $2 \mathrm{MeV} / \mathrm{cm}$ peak.

Figure $6.37 \mathrm{c}$ shows that the signal distribution is peaked around $2 \mathrm{MeV} / \mathrm{cm}$, as expected. The beam intrinsic $\mathrm{NC}$ component has a second peak around $4 \mathrm{MeV} / \mathrm{cm}$, mainly caused by $\pi^{0} \rightarrow 2 \gamma$ decays. Figure 6.37 a shows that electromagnetic showers originated by photons have a peak around $4 \mathrm{MeV} / \mathrm{cm}$ and a second peak around $2 \mathrm{MeV} / \mathrm{cm}$, as explained in Section 6.3.6. 
The POT-normalised plots (Figure 6.37b, 6.37a) show a good agreement between data and Monte Carlo.

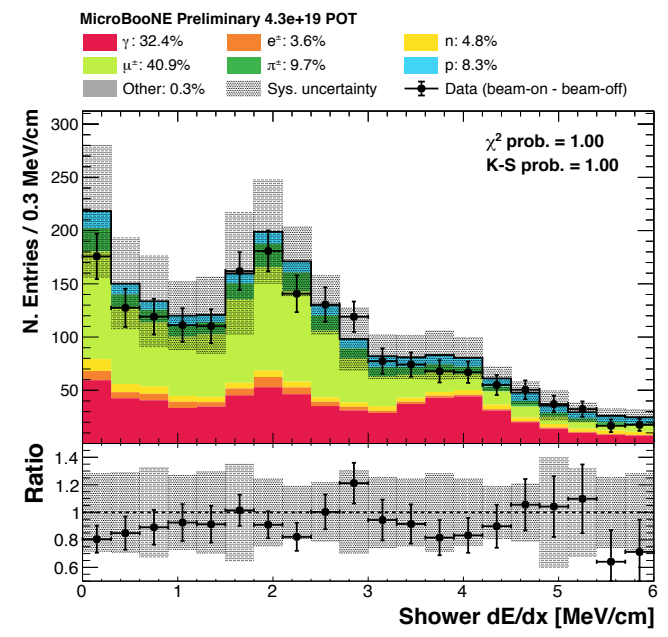

(a) POT-normalised, generating particle.

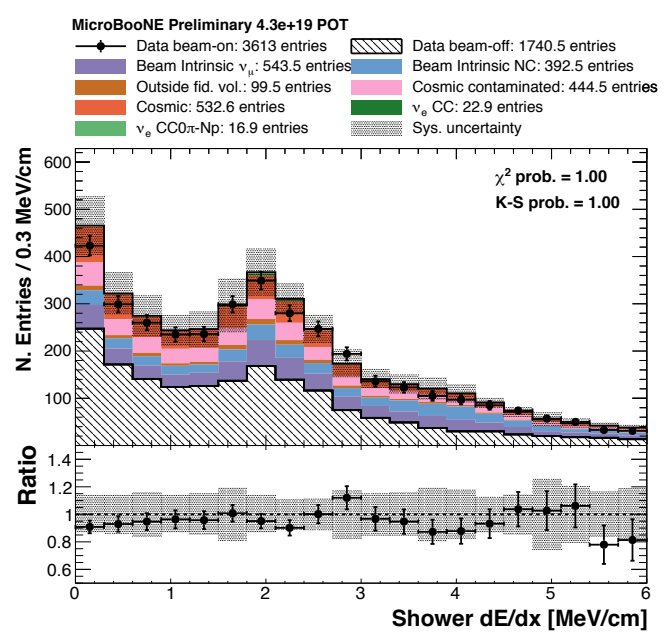

(b) POT-normalised, event category.

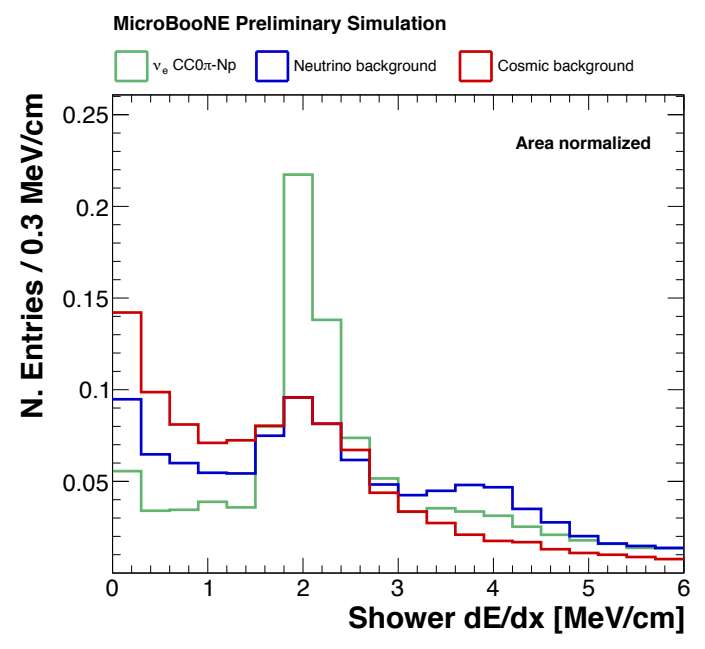

(c) Area-normalised.

Figure 6.37: Area and POT-normalised distributions for reconstructed showers $d E / d x$, classified according to the primary particle generating the shower (left) and to the event category (right). In the generating-particle histogram, the data points correspond to the statistical subtraction of the data beam-off events from the data beam-on events.

\section{Track distance $d_{t}<5 \mathrm{~cm}$}

A well-reconstructed event with a proton in the final state will have a reconstructed track attached to the reconstructed neutrino vertex. The track with the lowest proton $\chi_{p}^{2}$ score is required to be within $5 \mathrm{~cm}$ of the reconstructed neutrino vertex. This conservative cut can be tightened as the understanding of the spatial resolution 
improves. Figure 6.38c shows that the distributions of the distance between the start point of the reconstructed tracks and the reconstructed neutrino vertex for signal and background are very similar. The cut $d_{t}<5 \mathrm{~cm}$, then, mainly ensures that the event is well reconstructed. There is a slight disagreement in the first bin of Figures 6.38a and 6.38b, which is caused by a mis-modelling of the charge induced on neighbouring wires. This effect is taken into account by the Induced charge detector systematic sample (described in Section 7.4.)

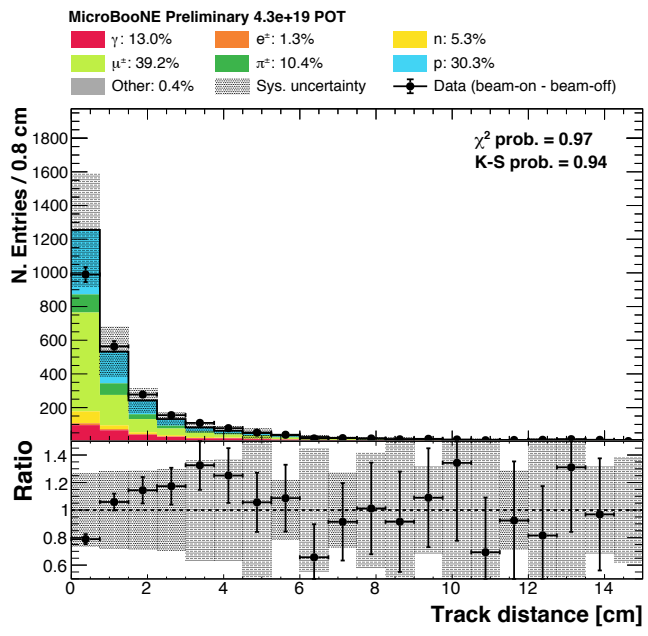

(a) POT-normalised, generating particle.

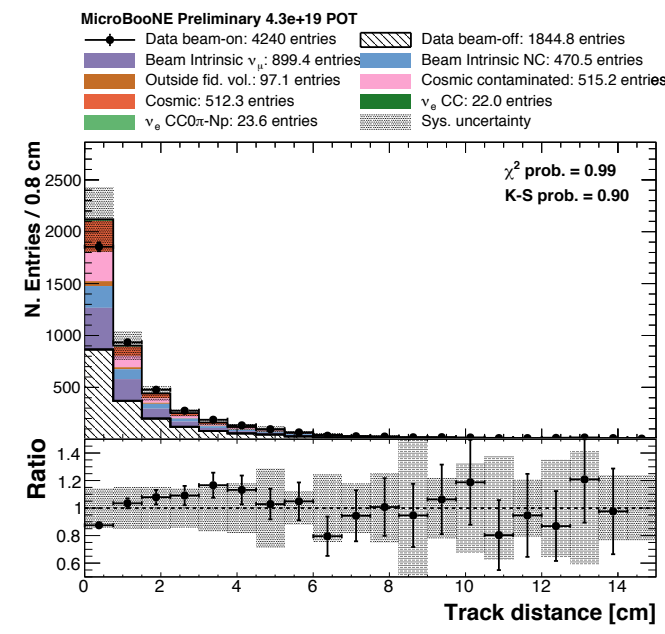

(b) POT-normalised, event category.

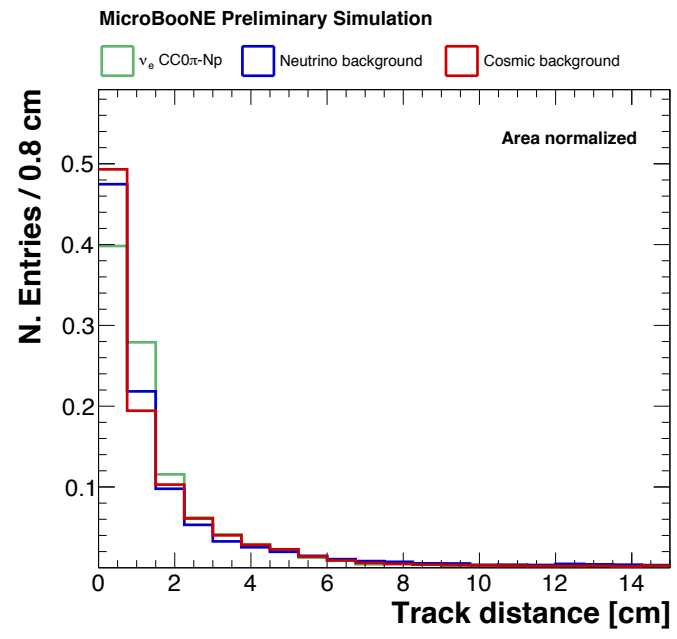

(c) Area-normalised.

Figure 6.38: Area (bottom) and POT-normalised distributions of the distance between the reconstructed tracks and the reconstructed neutrino vertex, classified according to the primary particle generating the shower (top left) and to the event category (top right). 


\section{Shower distance $d_{s}<5 \mathrm{~cm}$}

Shower initiated by a photon will have a gap between the interaction vertex and the start of the shower, given the interaction length in liquid argon $X_{0}=14 \mathrm{~cm}$. In order to suppress events with a photon in the final state, the most energetic shower starting point is required to be within $5 \mathrm{~cm}$ of the reconstructed neutrino vertex. Figure 6.39c shows the distributions of the distance between the start point of the reconstructed showers and the reconstructed neutrino vertex for signal and background events. As expected, background neutrino events (with photons) have a slightly larger tail than the signal events (with electrons). The agreement between data and Monte Carlo shown in Figure 6.39b and Figure 6.39a is good. Improvements currently implemented in the Pandora framework will allow for more appropriate cuts to further reduce the photon background.

\section{Track proton $\chi_{p}^{2}<80$}

It is possible to perform a $\chi^{2}$ test on the $d E / d x$ vs. the residual range of the reconstructed track under the hypothesis of a proton stopping in the detector, using the parametrisation of Section 6.3.7. Low values of the $\chi_{p}^{2}$ score will correspond to proton-like tracks, while a high value will correspond to a MIP-like track. Figure $6.40 \mathrm{c}$ shows the distributions of the $\chi_{p}^{2}$ score for background and signal events. Figure $6.40 \mathrm{a}$ shows that the protons reconstructed as tracks are peaked around 0 as expected, while the long tail includes muon tracks, photon and electron showers misclassified as tracks, and objects with a misplaced vertex. The comparison between data and Monte Carlo in Figure 6.40a and Figure 6.40b shows some discrepancies, especially for very low and very high $\chi_{p}^{2}$ scores. This quantity requires a careful simulation of the signal processing and a correct evaluation of the recombination effect. Our cut is in a region with a good data/Monte Carlo agreement once the systematic uncertainties are taken into account and it is as such deemed safe. 


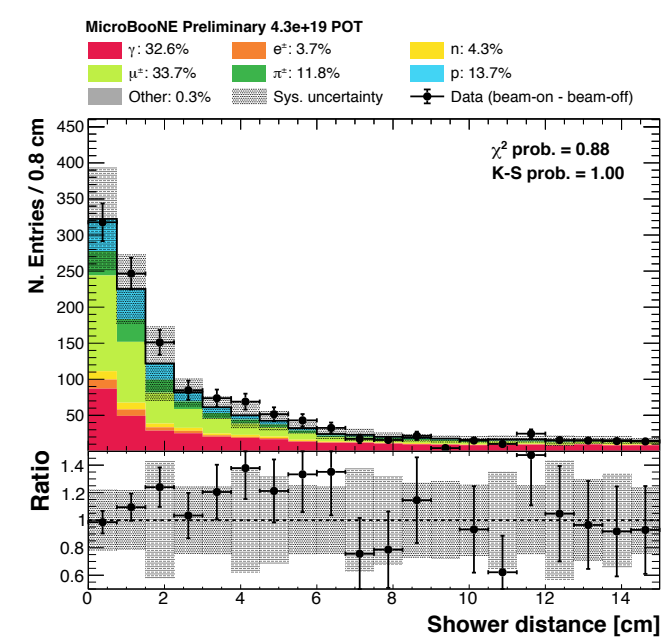

(a) POT-normalised, generating particle.

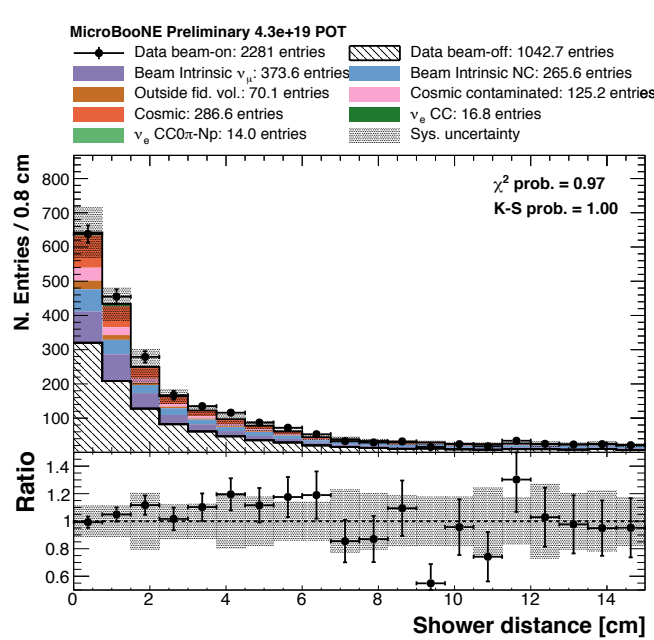

(b) POT-normalised, event category.

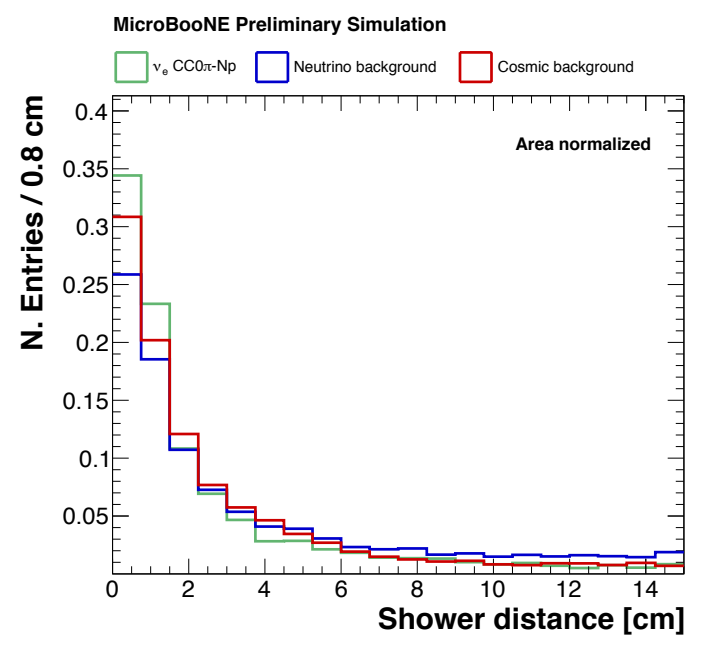

(c) Area-normalised.

Figure 6.39: Area (bottom) and POT-normalised distributions of the distance between the reconstructed showers and the reconstructed neutrino vertex, classified according to the primary particle generating the shower (top left) and to the event category (top right).

\section{Track-shower angle $\cos \alpha>-0.95$}

Electrons start producing an appreciable shower in the detector after several centimetres. In this case, the pattern recognition often identifies the first part of the shower as a track-like object and the latter part of the shower as a showerlike object. Furthermore, high-energy cosmic rays can produce a shower in the detector, which will be mostly aligned to a cosmic muon track. In order to remove these mis-reconstructed events and reduce this kind of cosmogenic background we require $\cos \alpha>-0.95$, where $\alpha$ is the angle between the most energetic shower 


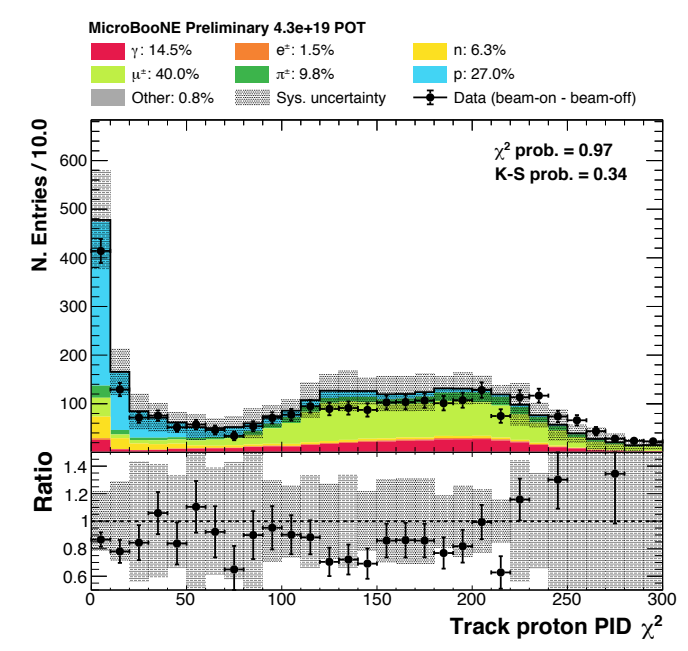

(a) POT-normalised, generating particle.

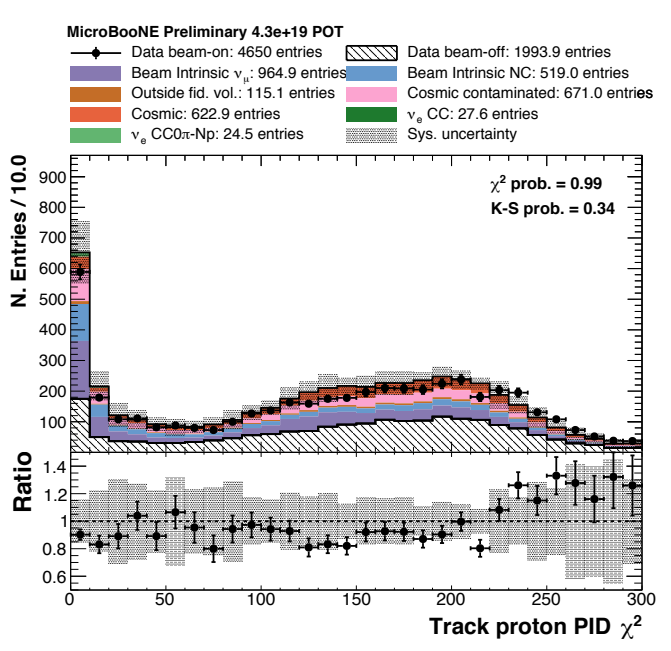

(b) POT-normalised, event category.

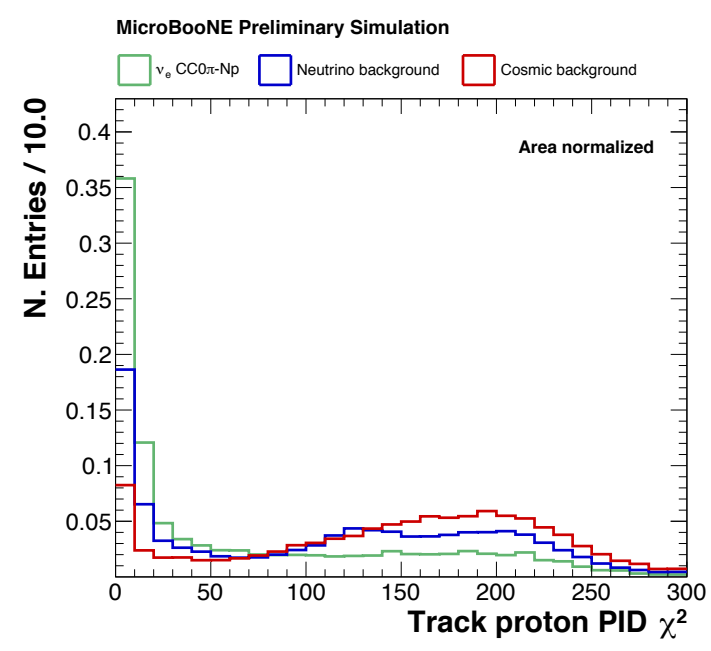

(c) Area-normalised.

Figure 6.40: Area (bottom) and POT-normalised distributions of the proton $\chi_{p}^{2}$ score of the reconstructed tracks, classified according to the primary particle generating the shower (top left) and to the event category (top right).

and the track with the lowest proton $\chi_{p}^{2}$ score. Figure $6.41 \mathrm{c}$ shows that there are, in proportion, more background events with a high angular separation between the tracks and the most energetic shower. This cut allows to reject these events while also ensuring that the signal events are well-reconstructed. In fact, signal events with $\cos \alpha \approx-1$ have almost always an electron shower reconstructed as a track-like object in the first part. The agreement shown in Figure 6.41b and Figure 6.41a is good. Future improvements in the shower reconstruction will allow for an increased selection efficiency. 


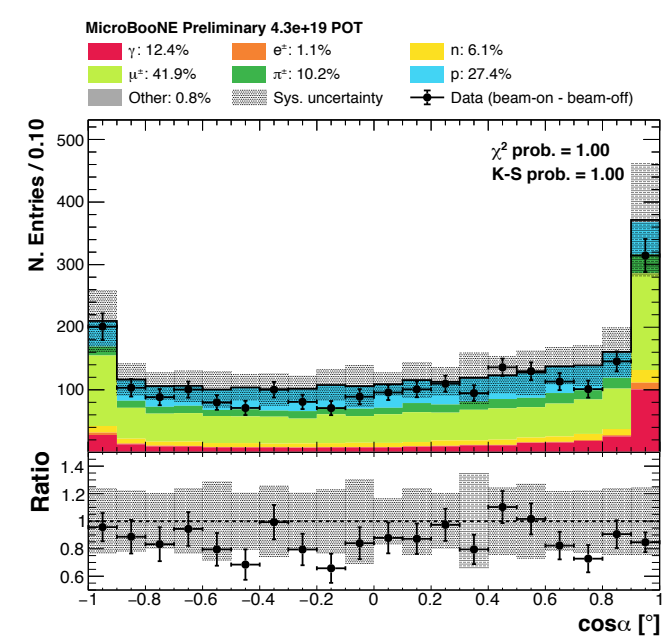

(a) POT-normalised, generating particle.

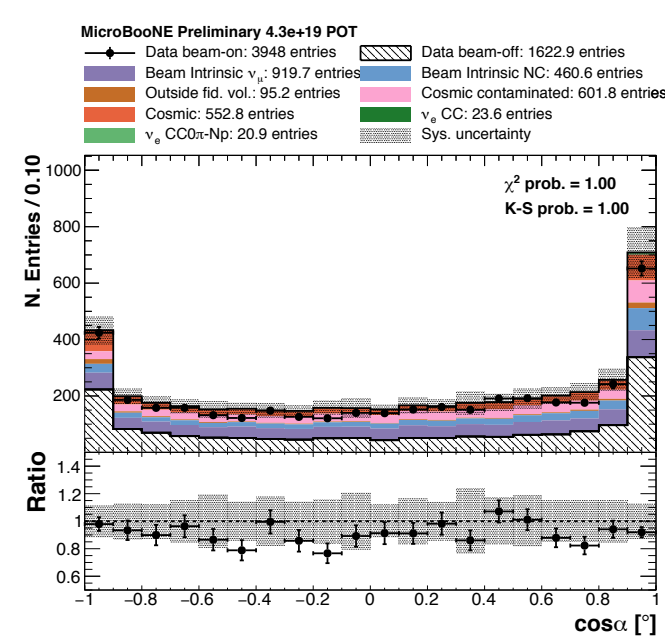

(b) POT-normalised, event category.

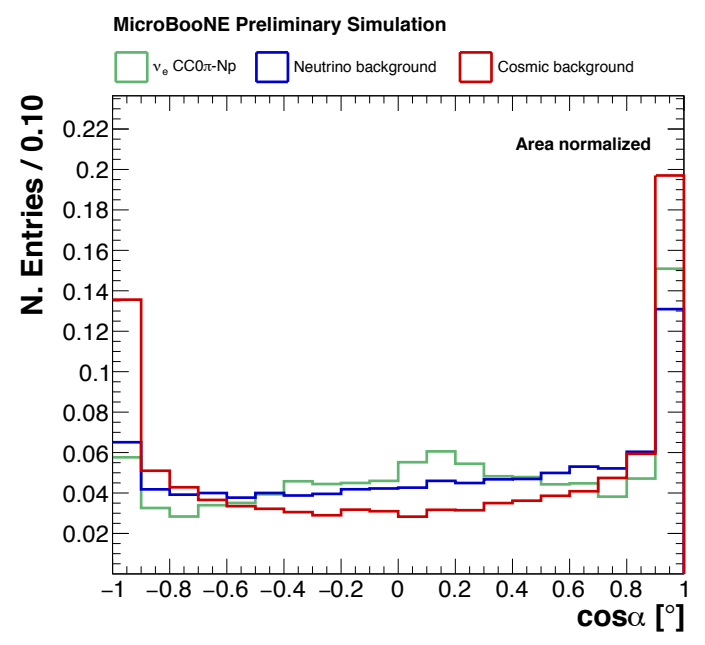

(c) Area-normalised.

Figure 6.41: Area (bottom) and POT-normalised distributions of the angle $\alpha$ between each reconstructed track and the leading shower, classified according to the primary particle generating the shower (top left) and to the event category (top right).

\section{Most proton-like track length $L<80 \mathrm{~cm}$}

The tracks in our $\nu_{e} \mathrm{CC} 0 \pi-\mathrm{Np}$ sample should correspond to protons. Protons in liquid argon have a higher stopping power than muons, which will correspond on average to shorter tracks. The track with the lowest $\chi_{p}^{2}$ proton score is required to be shorter than $80 \mathrm{~cm}$. This cut helps to reject mainly CC $\nu_{\mu}$ events with high-energy muons in the final state. Both neutrino and cosmic background events have on average longer reconstructed tracks than signal events, as shown in Figure 6.42c. The cut $L<80 \mathrm{~cm}$ increases the signal purity without significantly affecting 
the signal efficiency. The agreement between data and Monte Carlo distributions is good (Figures 6.42a, 6.42b).

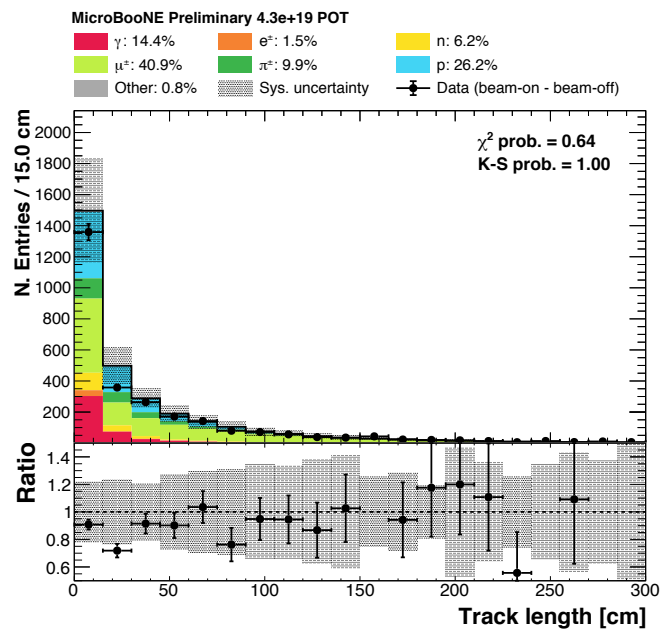

(a) POT-normalised, generating particle.

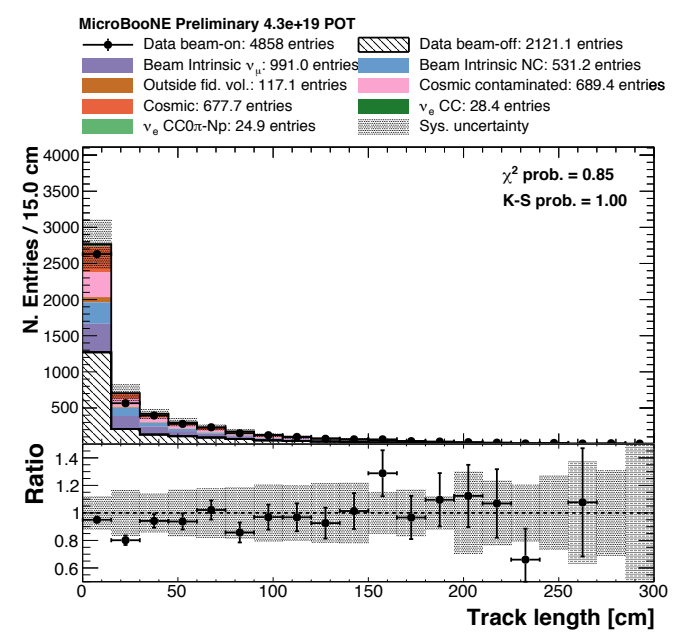

(b) POT-normalised, event category.

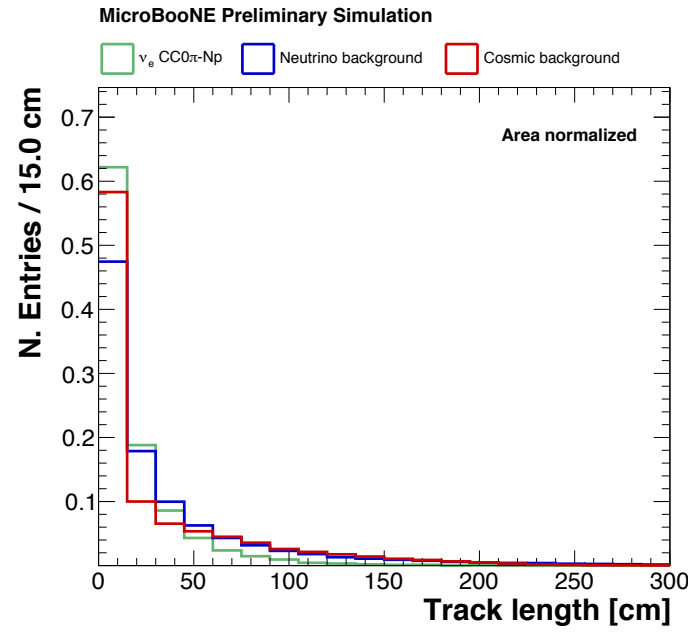

(c) Area-normalised.

Figure 6.42: Area (bottom) and POT-normalised distributions of the reconstructed tracks, classified according to the primary particle generating the shower (top left) and to the event category (top right).

\section{Rectangular cuts selection results}

The goal of the rectangular cuts is to isolate the $\nu_{e} \mathrm{CC} 0 \pi-\mathrm{Np}$ events and increase the purity of our selected sample. However, in order to validate our cuts and verify the agreement between data and Monte Carlo after this stage, it is necessary to select a sufficient number of data events. As mentioned before, in this analysis 
we require at least one data event in the bins in the $[0.2,1.9] \mathrm{GeV}$ range, which is the energy region we are most interested into.

We select 16 data beam-on events, $2.8 \pm 0.6$ beam-off events, and $12.4 \pm 3.0$ simulated events (including $\nu_{e}$ and $\nu_{\mu}$ interactions), corresponding to $4.34 \times 10^{19} \mathrm{POT}$. The number of selected $\nu_{e} \mathrm{CC} 0 \pi$-Np events is $3.2 \pm 0.8$, which gives a final efficiency of $(10.0 \pm 0.3$ (stat) \pm 0.5 (sys) $) \%$.

The purity of the sample is defined as:

$$
P=\frac{\mathrm{N} \text {. of selected } \nu_{\mathrm{e}} \mathrm{CC} 0 \pi-\mathrm{Np} \text { events }}{\mathrm{N} . \text { of selected events }} .
$$

Figure 6.43 shows the purity before and after the application of the rectangular cuts as a function of the reconstructed energy $E_{\text {deposited }}$. It increases by a factor of 40 , from $0.5 \%$ to $21.2 \%$.

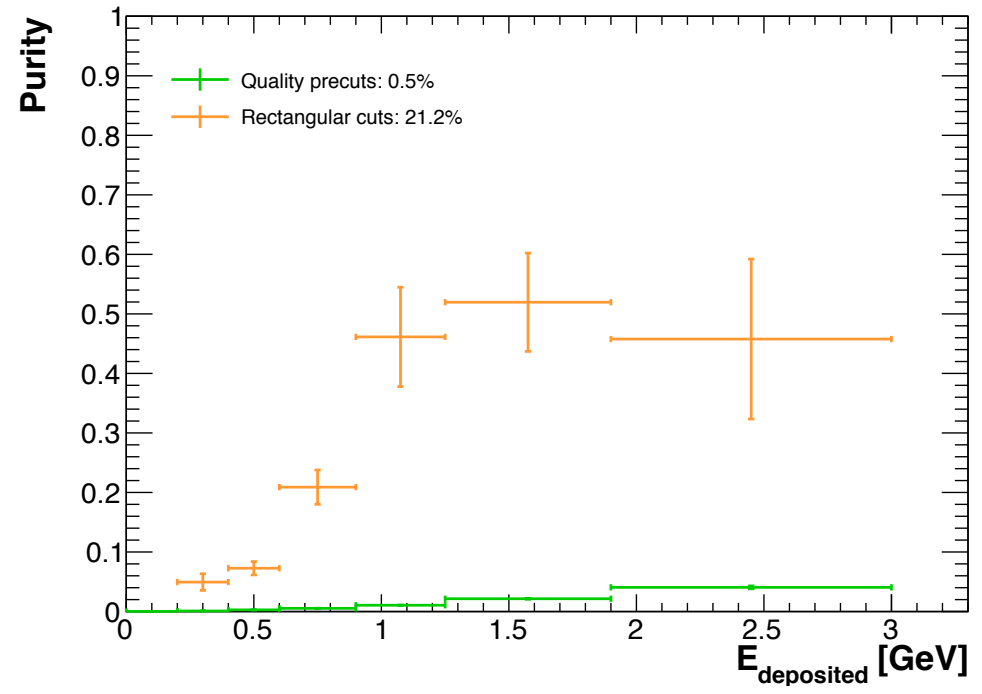

Figure 6.43: Purity of the selected sample before (green) and after (orange) the application of the rectangular cuts as a function of the reconstructed energy $E_{\text {deposited }}$. Error bars are statistical only.

It is also possible to calculate the overall purity and the efficiency after each cut, to analyse its effect (Figure 6.44). The largest purity increase is given by the application of the proton $\chi_{p}^{2}$ score cut.

Figure 6.45 shows the reconstructed energy spectrum $E_{\text {deposited }}$ after the application of the rectangular cuts. The data and simulation agree both in shape and in normalisation. 


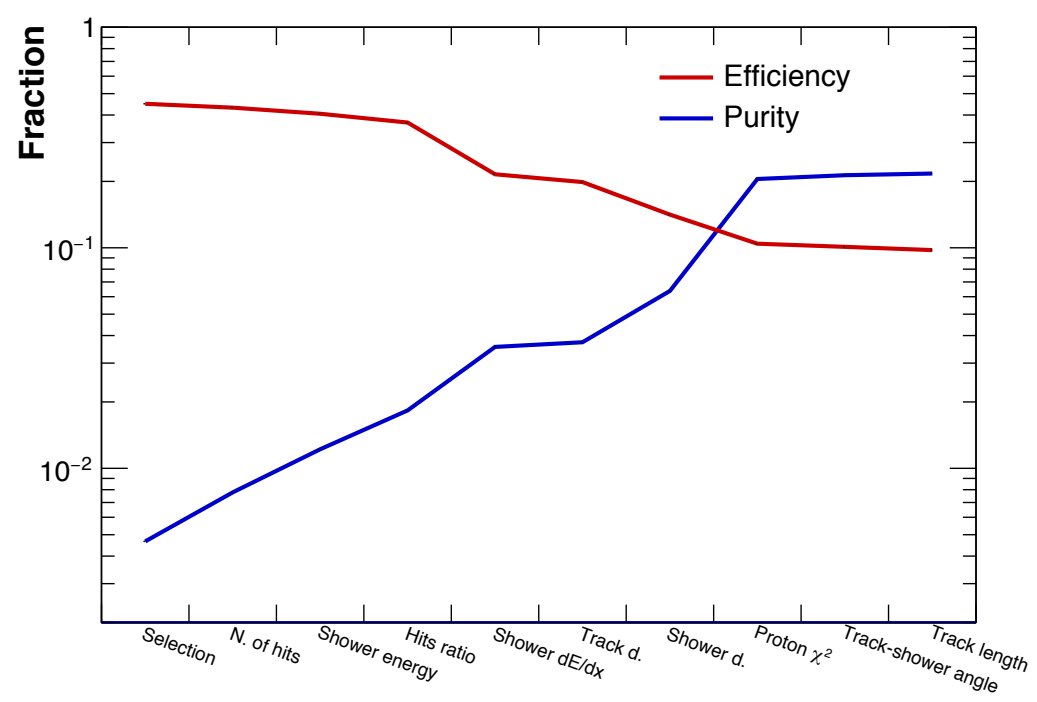

Figure 6.44: Selection efficiency (red) and purity (blue) of the selected sample after the application of each rectangular cut.

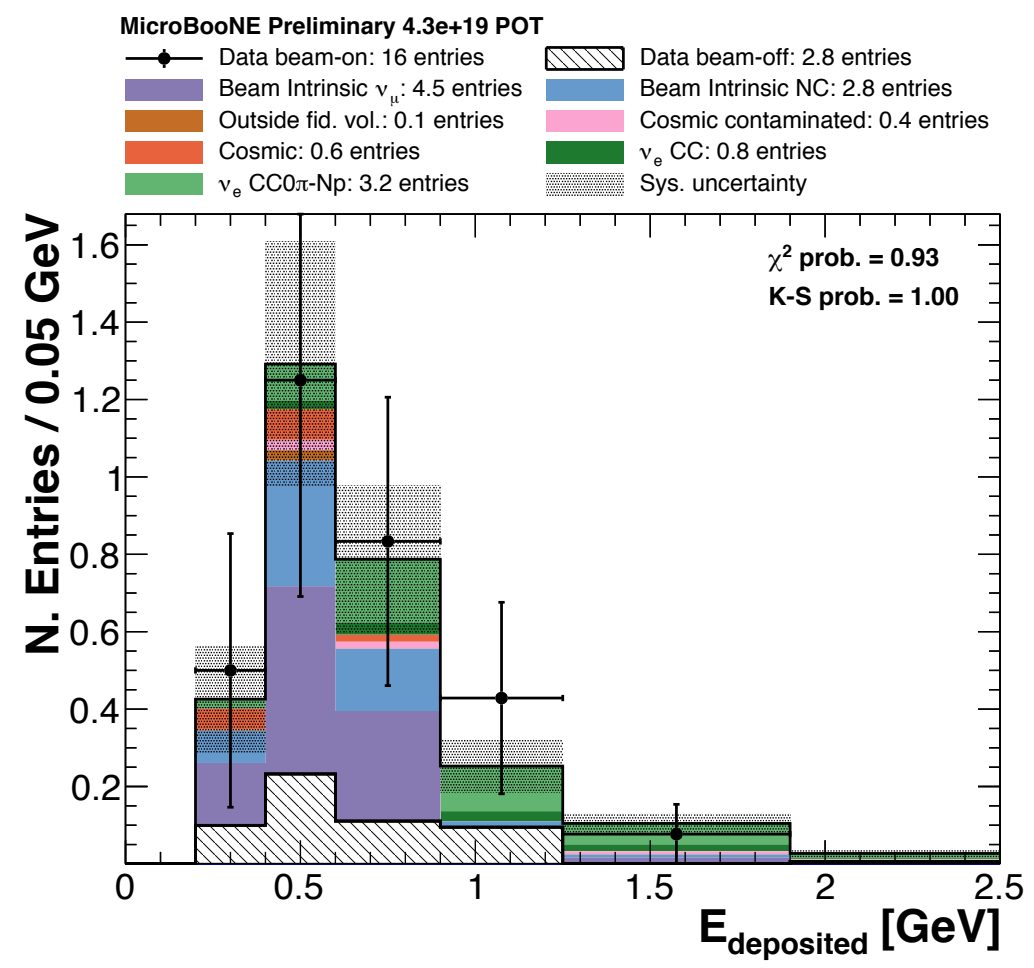

Figure 6.45: Reconstructed energy spectrum $E_{\text {deposited }}$ after the application of the rectangular cuts. The black points represent the data with statistical uncertainties. The coloured stacked histograms represent the simulated events, with the hatched histogram corresponding to the data beam-off sample. The shaded area represents the systematic uncertainty. 


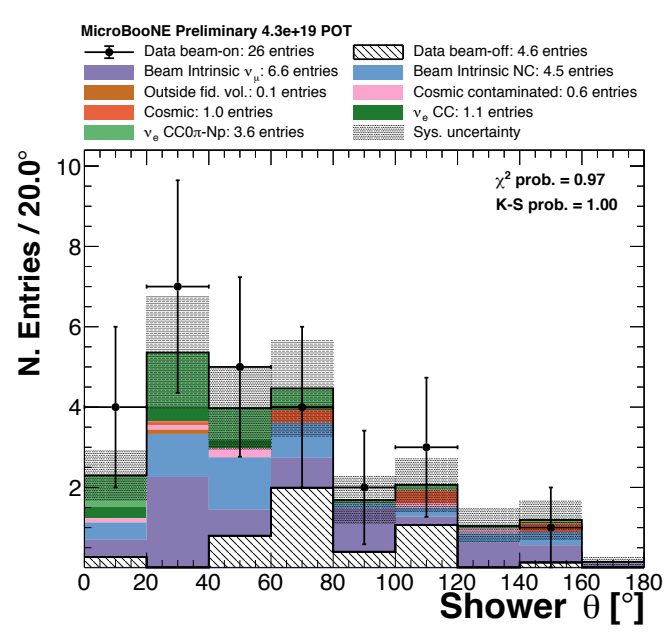

(a) $\theta$ angle.

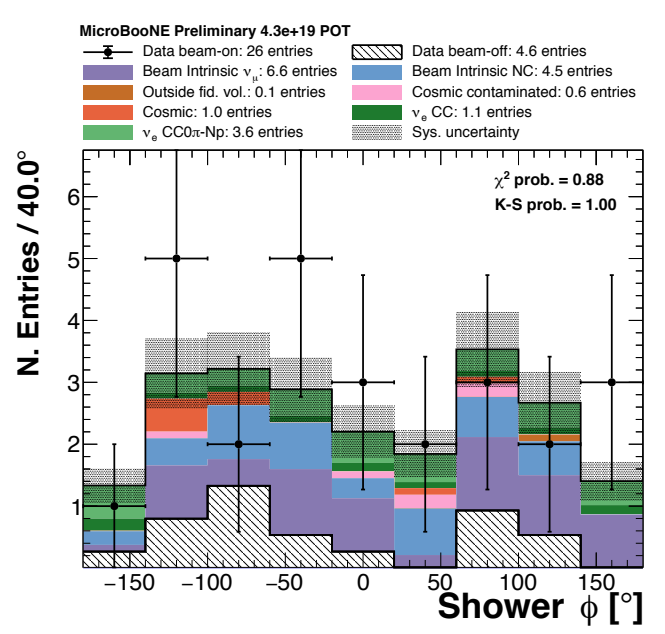

(b) $\phi$ angle.

Figure 6.46: Angular distribution of the selected Monte Carlo and data events after the application of the rectangular cuts. The black points represent the data with statistical uncertainties. The coloured stacked histograms represent the simulated events, with the hatched histogram corresponding to the data beam-off sample. The shaded area represents the systematic uncertainty.

The angular distributions of the reconstructed showers are shown in Figure 6.46 As expected, the reconstructed showers are peaked at low values of the $\theta$ angle, since the interactions are forward-boosted, and equally distributed on the $\phi$ angle. The agreement between data and Monte Carlo is good.

It is also possible to take a look at selected background events, in order to check where our rectangular cuts failed. Figure 6.47a shows a $\mathrm{CC} \pi^{0}$ selected event, which belongs to the Beam intrinsic $\nu_{\mu}$ category. In this case, the muon in the final state of the $\mathrm{CC}$ interaction stops after $\sim 20 \mathrm{~cm}$ and decays producing a Michel electron. The muon was classified by the pattern recognition as a shower: its $d E / d x$ is around $2 \mathrm{MeV} / \mathrm{cm}$ and it starts ionising at the interaction vertex, so it is not rejected by the shower-gap cut. Figure $6.47 \mathrm{~b}$ shows a selected $\mathrm{NC} \pi^{0}$ event (Beam intrinsic NC category). Here, one photon from the $\pi^{0} \rightarrow \gamma \gamma$ decay pair-produced within $3 \mathrm{~mm}$ the interaction vertex, so it shows as a shower attached to the interaction vertex. The pair-production was also very asymmetric, so the measured $d E / d x$ was around $2 \mathrm{MeV} / \mathrm{cm}$.

Both these events, which represent a small fraction of the simulated events passing our rectangular cuts, could be rejected by applying a cut on the number 


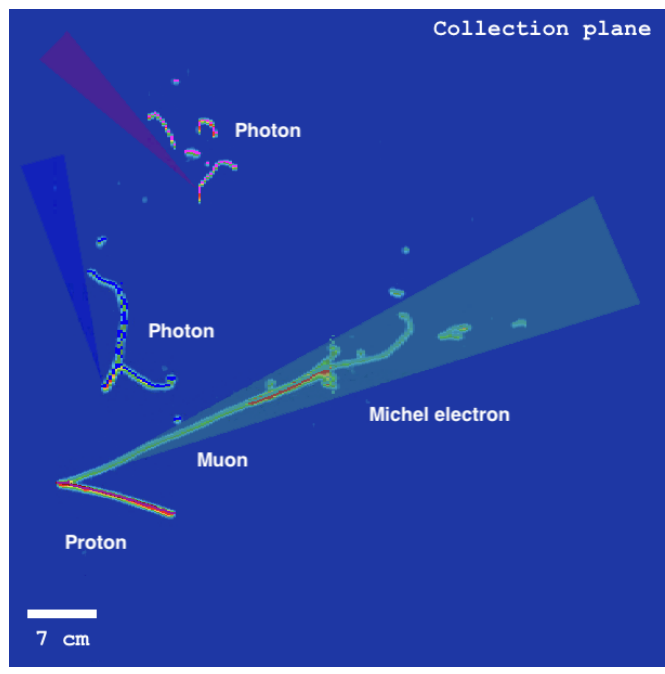

(a) Simulated $\mathrm{CC} \pi^{0}$ event

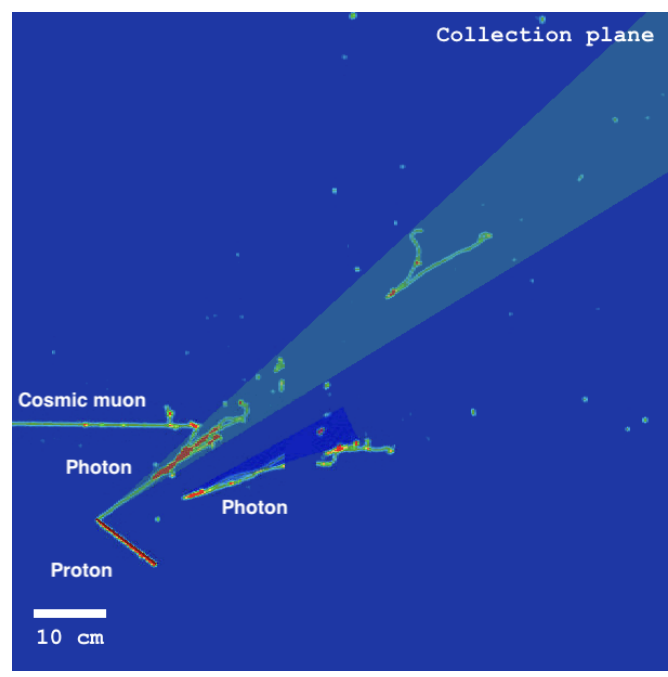

(b) Simulated $\mathrm{NC} \pi^{0}$ event.

Figure 6.47: Event displays of the collection plane of background events not rejected by the rectangular cuts listed in Section 6.4.1

of reconstructed showers or a cut on the $d E / d x$ of all the reconstructed showers. However, the pattern recognition tends to split also legit electron showers, so this kind of cuts would sensibly affect also our $\nu_{e} \mathrm{CC} 0 \pi$-Np selection efficiency.

\subsubsection{Boosted Decision Trees}

Selecting signal events with rectangular cuts on kinematic and calorimetric variables allows to assess with great precision the effect of every single cut. It is therefore a very clear approach and easy to cross-check. However, it presents several limitations: many signal events may fail only a single cut and still being rejected and it is difficult to optimise the combination of a large number of cuts.

A natural extension to the rectangular cuts approach is represented by the application of a decision tree, widely used in social sciences and also in highenergy physics. In particular, the MiniBooNE collaboration showed that Boosted Decision Trees (BDTs) could be used for particle identification in 116 , and the D0 collaboration used BDTs for the search of single top production in [117. A classical decision tree algorithm can be divided into four stages, as listed in 118 and described below.

1. Sort signal and background events by each variable. 
2. Select the variable and splitting value with the best separation.

3. Produce two nodes, one containing the events passing the selection and one the events failing the selection. Each node has a defined purity, measured as the fraction of signal events in the node.

4. Repeat iteratively until the stopping condition (described below) is reached. The same variable can be used multiple times. The terminal node is called signal leaf if it contains mostly signal events or background leaf if it contains mostly background events.

In this analysis, the separation is measured using the Gini coefficient $G$, defined as:

$$
G=P(1-P) \sum_{i} W_{i}
$$

where $P$ is the purity of the node and $W_{i}$ is the weight of the $i$ event. The best separation is chosen as the one which minimises the $G_{\mathrm{x}}+G_{\mathrm{y}}$ sum, where $x$ and $y$ are the two new nodes of the tree.

The decision to stop the iteration (the stopping condition) can depend on multiple conditions: (1) a minimum size of the leaf can be required for statistical significance, (2) the events are perfectly classified (purity of the leaf is 1 or 0 ), (3) the purity cannot be improved with any choice of the splitting value, or (4) the tree depth reaches a maximum value. Usually, the decision tree is produced with a set of simulated events called training sample and then applied to an independent set called test sample. For a single event in the test sample, the decision tree score corresponds to the purity of the leaf where the event ends up.

The use of a decision tree to perform background rejection presents several advantages:

- it is not affected by the curse of dimensionality: the computing consumption scales only linearly with the number of variables used;

- the result is insensitive to duplicate variables; 
- the presence of very similar variables does not decrease the classification power;

- the order of the training events is irrelevant;

- the result is insensitive to the transformation of the variables with any strictly monotone function (it will produce the same event ordering and, as such, the same decision tree).

A disadvantage of a classical decision tree is its intrinsic instability: a small change in the training sample can produce wildly different branches and leaves. In order to solve this problem, the so-called boosting technique was introduced. In the boosting, training events which are misclassified (a signal event ends up in a background leaf or vice-versa) get assigned an increased weight and a new tree is formed. This process is repeated iteratively and new $m$ trees are created. The score of the individual $k$ tree $T_{k}$ is taken as 1 if the event ends up in a signal leaf, or -1 if the event ends up in a background leaf. The final score $T(i)$ of the event $i$ is taken as the weighted sum of the scores of the individual trees:

$$
T(i)=\frac{1}{\sum_{k=1}^{m} \alpha_{k}} \sum_{k=1}^{m} \alpha_{k} T_{k}(i) .
$$

In this document, we will use the adaptive boosting, or AdaBoost [119. In this algorithm, the $\alpha$ coefficient is defined as:

$$
\alpha=\beta \log [(1-\epsilon) / \epsilon]
$$

where $\epsilon$ is the weighted sum of the misclassified events and $\beta$ is constant (in our case is set at 0.5$)$. Figure 6.48 shows function 6.22 for different values of $\beta$. The events in the $k$ tree have their weight multiplied by $e^{\alpha_{k}}$.

\section{Background rejection with Boosted Decision Trees}

In order to maximise our separation power, in this analysis we trained three different BDTs, each one tuned on a different background category: 


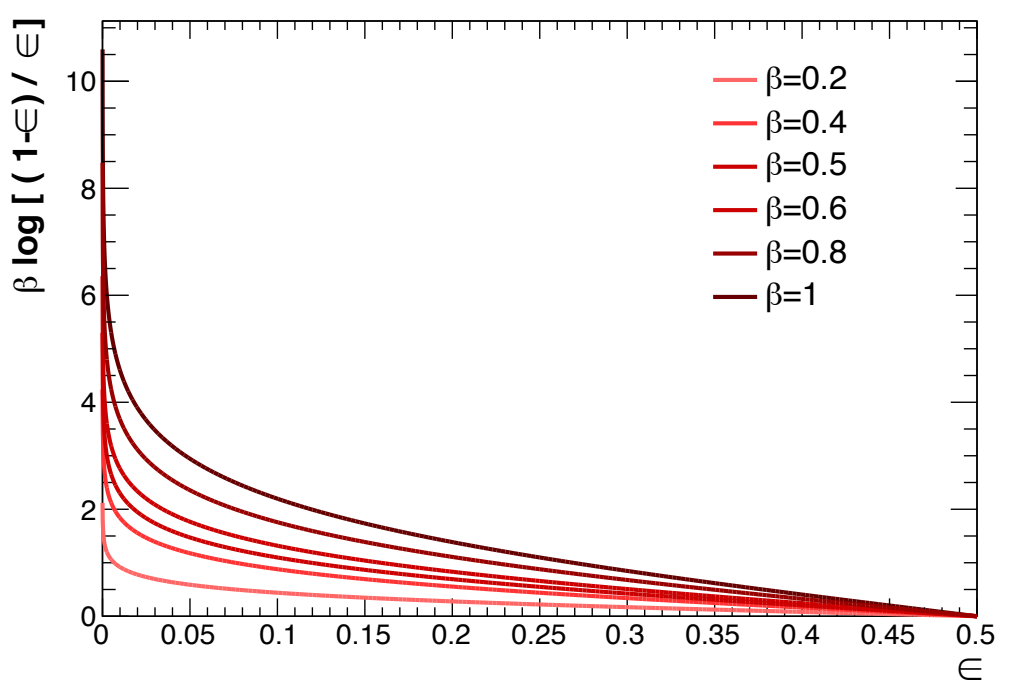

Figure 6.48: Coefficient function 6.22 of the AdaBoost boosting technique for different values of the $\beta$ parameter.

Cosmic BDT: trained with Cosmic, Cosmic contaminated and Data beam-off events as background and $\nu_{e} C C O \pi-N p$ events as signal.

Neutral-current BDT: trained with Beam intrinsic $N C$ events as background and $\nu_{e} C C O \pi-N p$ events as signal.

CC $\nu_{\mu}$ BDT: trained with Beam intrinsic $\nu_{\mu}$ events as background and $\nu_{e} C C 0 \pi$ $N p$ events as signal.

The variables used are the same in all three BDTs and they correspond to the ones described in Section 6.4.1. plus the angular and spatial distributions of the reconstructed tracks and showers. The BDT has been trained on 600 trees, using the Gini coefficient to measure the splitting power, requiring at least $5 \%$ of the training events in each terminal node, and using the AdaBoost boosting algorithm. The training has been performed with the TMVA toolkit 120].

The classification power of the BDTs can be compared by looking at the Receiver Operating Characteristic (ROC) curves, which show the background-rejection power as a function of the signal efficiency. Figure 6.49 shows the ROC curves for our three BDTs, in the case of samples with 1000 signal events and 1000 background events. The best-performing BDT is the Cosmic BDT. This is expected, since 
cosmic rays have greatly different angular, topological, and kinematic distributions when compared with neutrino interactions.

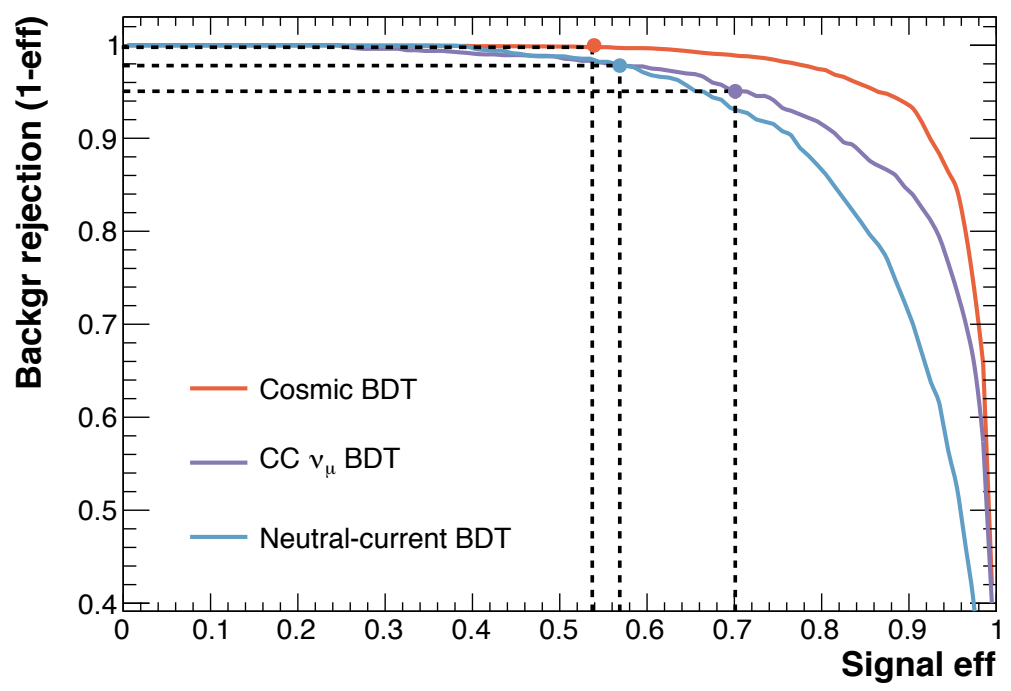

Figure 6.49: ROC curves for the three BDTs used in this analysis, showing the background-rejection power as a function of the signal efficiency in a sample with 1000 signal events and 1000 background events. The points correspond to the signal efficiency and background-rejection coordinates of the cut applied to each BDT score.

The least powerful BDT is the Neutral-current BDT. This is because NC events with $\pi^{0}$ production are the most difficult events to reject. As we have seen in Section 6.3.6, photons which undergo very asymmetric pair-production can have a $d E / d x$ around $2 \mathrm{MeV}$. When one of the photons of the $\pi^{0} \rightarrow \gamma \gamma$ decay escapes the detector or is not reconstructed, and the other one pair-produces asymmetrically near the interaction vertex, the event becomes basically indistinguishable from a $\nu_{e} \mathrm{CC} 0 \pi-\mathrm{Np}$ interaction.

The BDT is then applied to an independent Monte Carlo sample (the test sample) and to our data. It is very important to verify the agreement between the BDTs score distribution in data and Monte Carlo, since the presence of a bias in our training could create a fake excess.

Figure 6.50 shows the comparison between the data and Monte Carlo distributions for the Cosmic, Neutral-current, and CC $\nu_{\mu}$ BDTs. 


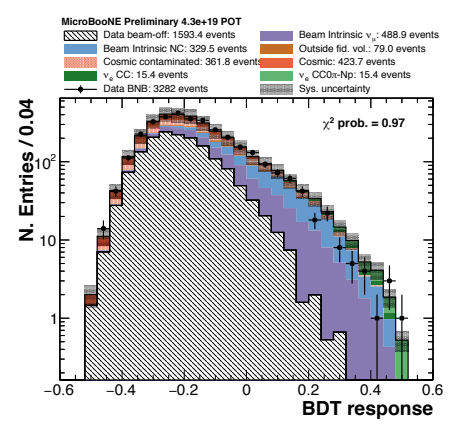

(a) Cosmic

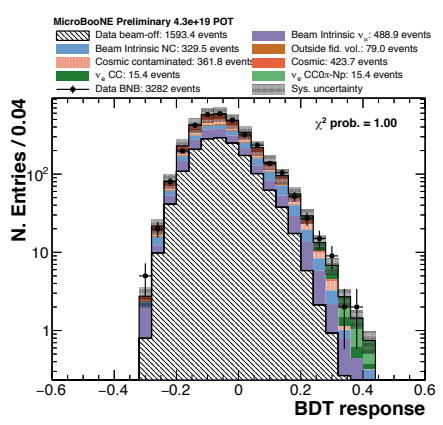

(b) Neutral-current

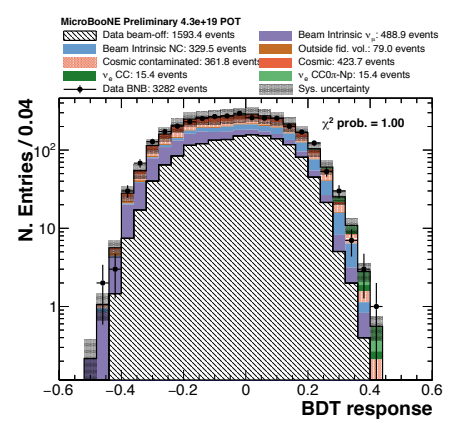

(c) $\mathrm{CC} \nu_{\mu}$

Figure 6.50: POT-normalised comparison between the data and Monte Carlo for distributions of the BDTs score. The black points represent the data with statistical uncertainties. The coloured stacked histograms represent the simulated events, with the hatched histogram corresponding to the data beam-off sample. The shaded area represents the systematic uncertainty. Vertical axis is in log-scale.

In order to maximise our $\nu_{e} \mathrm{CC} 0 \pi$-Np purity and have at least one event per bin in the $[0.2,1.9] \mathrm{GeV}$ range of the $E_{\text {deposited }}$ spectrum (same criterion used for the rectangular cuts), we applied the following cuts:

Cosmic $\mathrm{BDT}>0.21, \quad$ Neutral-current $\mathrm{BDT}>0.18, \quad \mathrm{CC} \nu_{\mu} \mathrm{BDT}>0.17 . \quad$ (6.23)

The background rejection power and the selection efficiency corresponding to each BDT cut are shown in Figure 6.49.

We select 11 data beam-on events, $0.5 \pm 0.13$ beam-off events, and $9.5 \pm 2.3$ simulated events (both $\nu_{e}$ and $\nu_{\mu}$ ), corresponding to $4.34 \times 10^{19}$ POT. The number of selected $\nu_{e} \mathrm{CC} 0 \pi$ - Np events is $4.4 \pm 1.2$, which corresponds to a final efficiency of $(13.0 \pm 0.3$ (stat) \pm 0.5 (sys) $) \%$. The final purity of the sample is $44.3 \%$, around twice the one obtained with the rectangular cuts (Figure 6.52). The final efficiency with the BDTs is $13.0 \%$, compared with the $9.5 \%$ obtained with the rectangular cuts. A comparison between the efficiency as a function of the true neutrino energy is shown in Figure 6.51. A summary of the selected events with both methods is shown in Table 6.3.

The distribution of the reconstructed energy spectrum $E_{\text {deposited }}$ after the application of the three BDT cuts is shown in Figure 6.53. The angular distributions of the reconstructed showers are shown in Figure 6.54. 


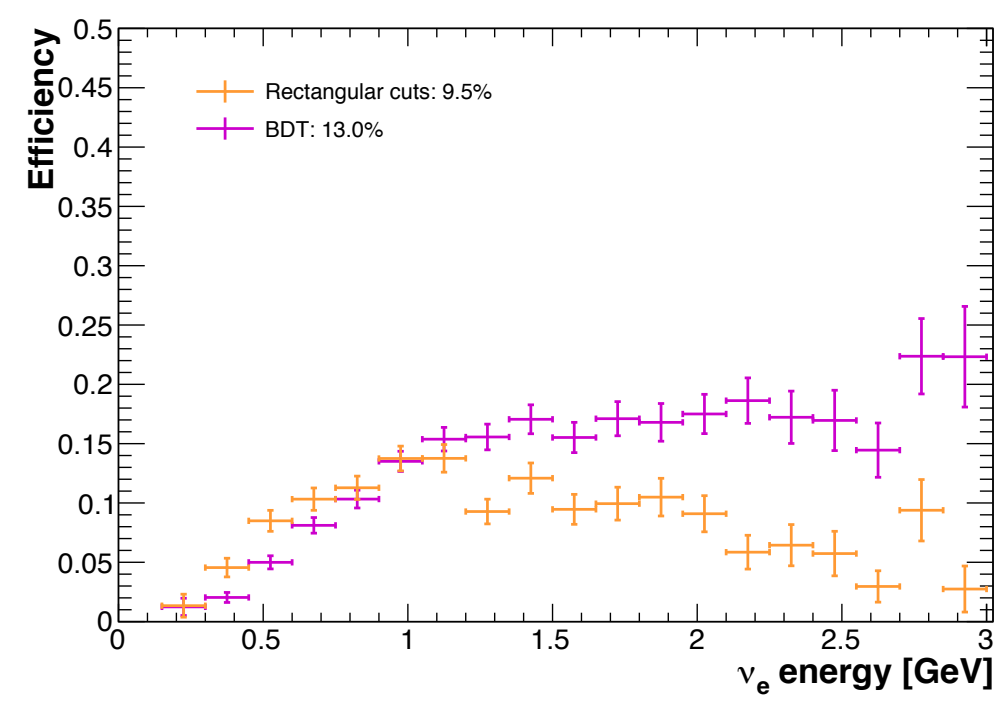

Figure 6.51: Selection efficiency as a function of true neutrino energy after the application of the rectangular cuts (orange), and after the application of the BDTs (purple). Error bars are statistical only.

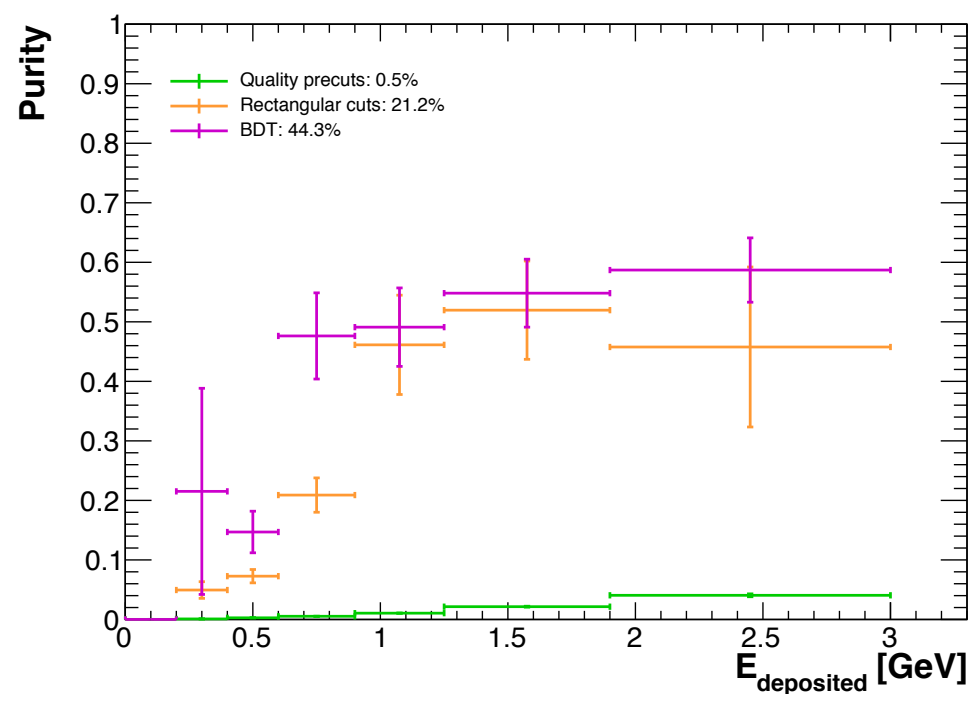

Figure 6.52: Purity of the sample after the event selection (green), after the application of the rectangular cuts (orange), and after the application of the BDTs (purple) as a

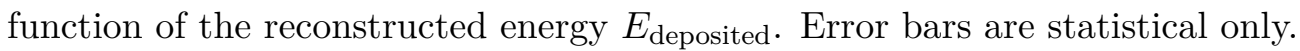


Table 6.3: Summary of selected events in simulation and data with rectangular and BDTs cuts for a MicroBooNE exposure of $4.34 \times 10^{19}$ POT. The uncertainties on the simulated events are caused by systematic effects described in Section 7 . Uncertainties on the data events are statistical only. Numbers are rounded up to the first decimal digit.

\begin{tabular}{lrr}
\hline Category & Rectangular & BDTs \\
\hline Data beam-on & $16 \pm 4$ & $11 \pm 3.3$ \\
Data beam-off & $2.8 \pm 0.6$ & $0.5 \pm 0.3$ \\
$\nu_{e}$ CC0 $\pi$-Np & $3.2 \pm 0.8$ & $4.4 \pm 1.2$ \\
$\nu_{e}$ CC & $0.8 \pm 0.2$ & $1.5 \pm 0.4$ \\
Beam intrinsic $\nu_{\mu}$ & $4.5 \pm 1.5$ & $1.7 \pm 0.6$ \\
Beam intrinsic NC & $2.8 \pm 0.9$ & $1.4 \pm 0.5$ \\
Outside fid. vol. & $0.1 \pm 0.0$ & $0.0 \pm 0.0$ \\
Cosmic & $0.6 \pm 0.1$ & $0.2 \pm 0.1$ \\
Cosmic contaminated & $0.4 \pm 0.1$ & $0.2 \pm 0.1$ \\
\hline Beam-on - beam-off & $13.2 \pm 4.0$ & $10.5 \pm 3.3$ \\
Total Monte Carlo & $12.4 \pm 3.0$ & $9.5 \pm 2.5$ \\
\hline
\end{tabular}

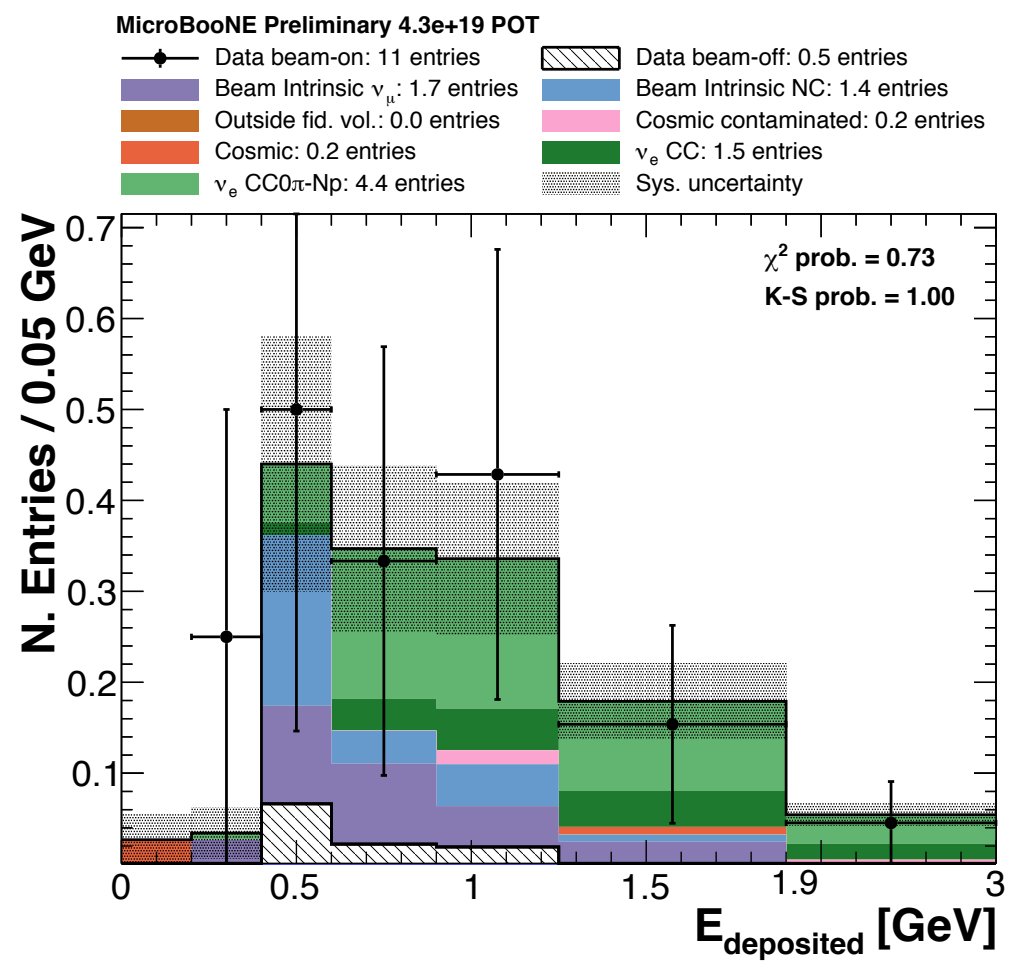

Figure 6.53: Reconstructed energy spectrum $E_{\text {deposited }}$ after the application of the BDTs cuts. The black points represent the data with statistical uncertainties. The coloured stacked histograms represent the simulated events, with the hatched histogram corresponding to the data beam-off sample. The shaded area represents the systematic uncertainty. 


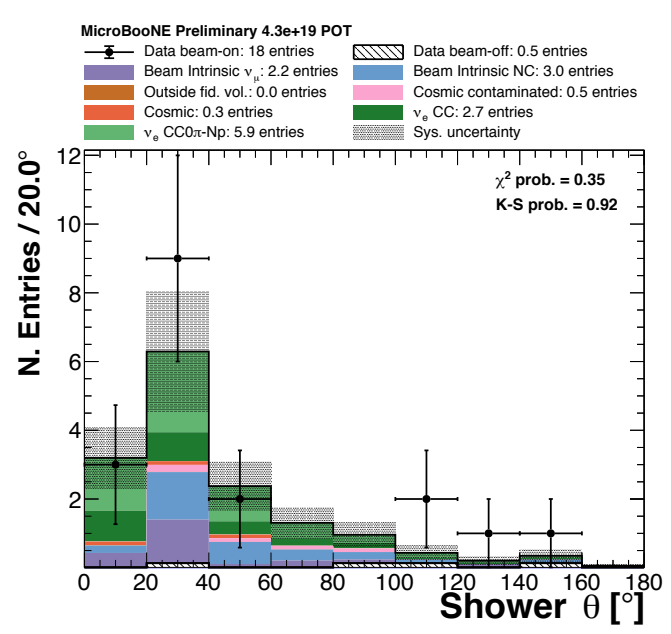

(a) $\theta$ angle.

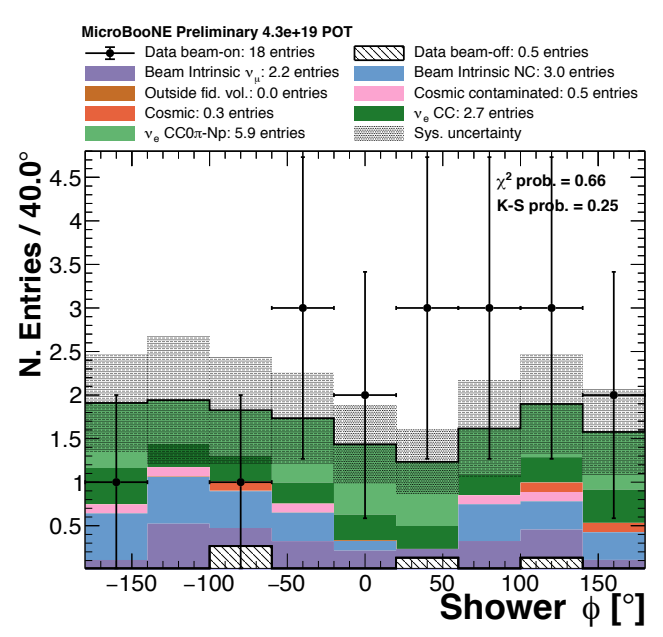

(b) $\phi$ angle.

Figure 6.54: Angular distribution of the selected Monte Carlo and data events after the application of the BDTs cuts. The black points represent the data with statistical uncertainties. The coloured stacked histograms represent the simulated events, with the hatched histogram corresponding to the data beam-off sample. The shaded area represents the systematic uncertainty.

\subsubsection{Interaction types}

It is also possible to categorise the selected events according to the neutrino interaction which produced the final-state particles. Figure 6.55 shows the selected events after the rectangular and BTDs cuts as a function of the GENIE interaction type. As expected, the majority of $\nu_{e} \mathrm{CC} 0 \pi$-Np events were produced by a quasielastic interaction, while the $\mathrm{NC}$ and $\mathrm{CC} \nu_{\mu}$ background come mainly from resonant interactions. A significant component of electron neutrinos also interacted via MesonExchange Current. This interaction involves the scatter between the neutrino and a correlated pair of nucleons and is accompanied by a 2-nucleon emission from the primary vertex (instead of a single nucleon emission from CCQE interactions). This phenomenological effect is often also called 2 particle-2 hole (2p-2h) [121.

\subsection{Validation}

\subsubsection{Electromagnetic shower energy loss}

A way to verify if we are effectively selecting electron neutrinos is to study the distribution of the electromagnetic showers energy loss per length $d E / d x$. If the 


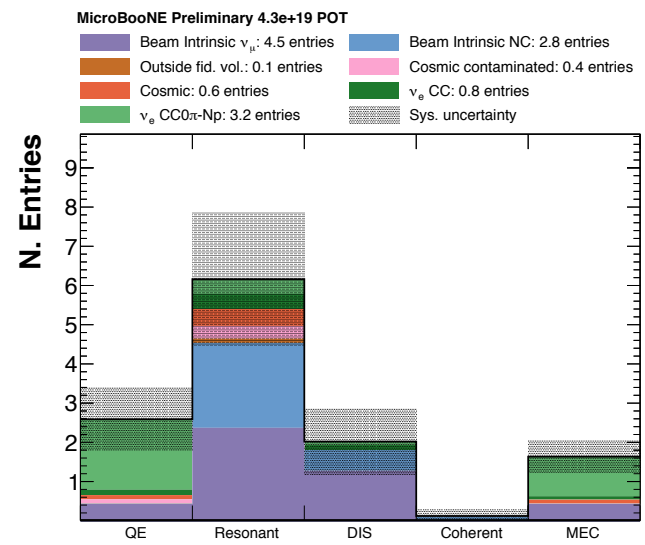

(a) Rectangular cuts.

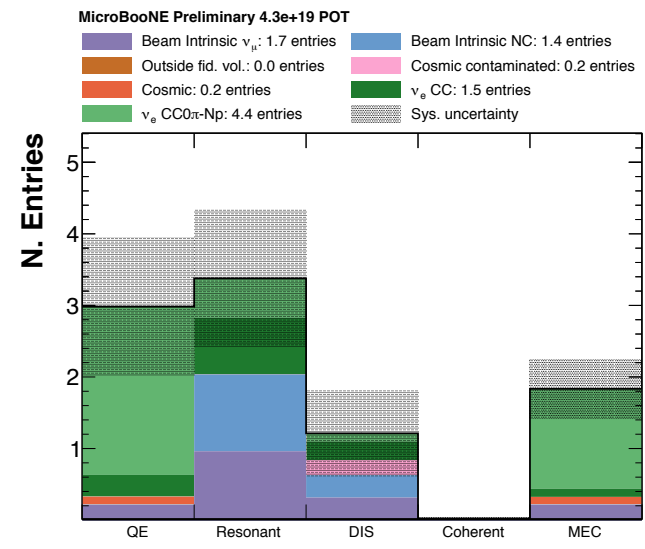

(b) BDTs cuts.

Figure 6.55: Selected events classified according to the type of neutrino interaction which generated them for different event type.

data events contain a well-reconstructed electron in the final state, the $d E / d x$ distribution will be peaked around $2 \mathrm{MeV} / \mathrm{cm}$. Figure 6.56 shows the $d E / d x$ distributions for data and Monte Carlo after the application of the rectangular cuts (left) and after the application of the BDTs cuts (right). As expected, the peak is in both cases around $2 \mathrm{MeV} / \mathrm{cm}$, meaning that the $d E / d x$ of the reconstructed showers in data are compatible with electrons in the final state.

\subsubsection{Side-bands checks}

In this section we will study the agreement between data and Monte Carlo for selected samples orthogonal to the $\nu_{e} \mathrm{CC} 0 \pi$-Np-enriched sample obtained in Section 6.4. In order to validate the analysis, some of the background-rejecting cuts are inverted or removed in order to enhance different background components.

\section{NC-enhanced selection}

It is possible to enhance the neutral-current component (defined as beam intrinsic $N C$ in our analysis) by (1) inverting the cut on the shower $d E / d x$, and (2) removing the cut on the shower distance (see Figures 6.37c, 6.39c). The $d E / d x$ of the most energetic shower must be within $3.2 \mathrm{MeV} / \mathrm{cm}$ and $5 \mathrm{MeV} / \mathrm{cm}$ to select electromagnetic cascades that were initiated by a photon. It also ensures that 


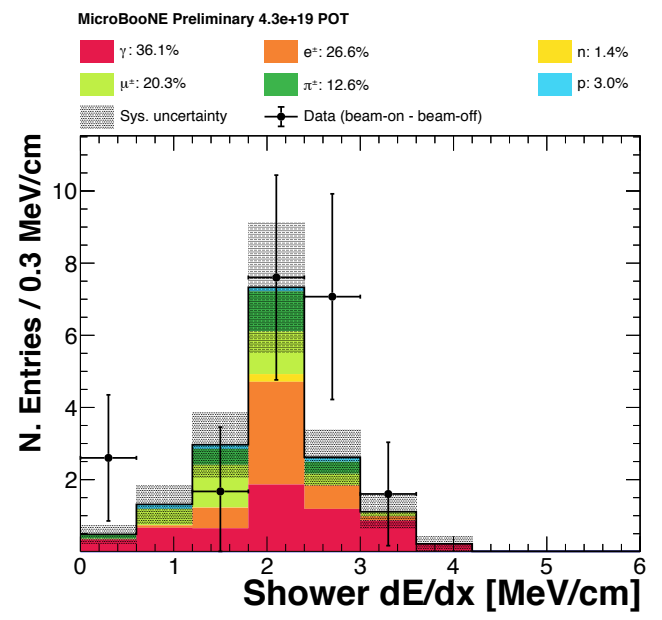

(a) Rectangular cuts.

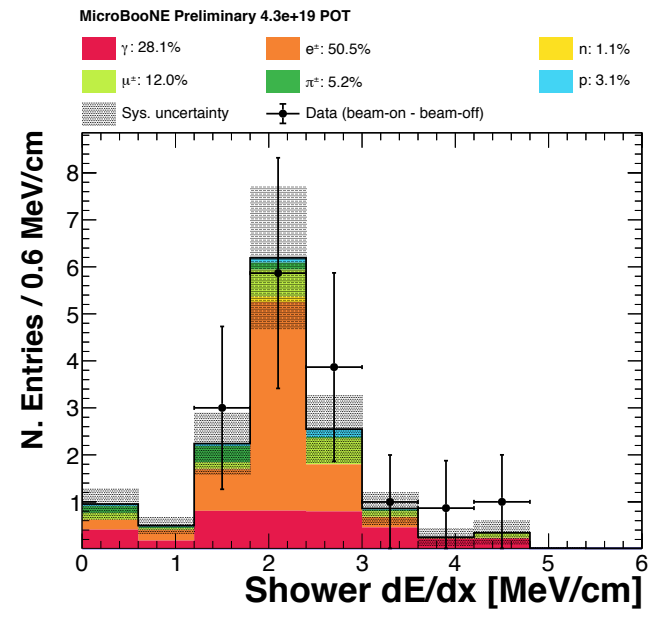

(b) BDTs.

Figure 6.56: Distribution of the reconstructed showers $d E / d x$ after rectangular cuts (left) and BDTs cuts (right). The black points represent the statistical subtraction of the data beam-off events from the data beam-on events. The coloured stacked histograms represent the simulated events, classified according to the particle which generated the shower.

this NC-enhanced sample is orthogonal to the $\nu_{e} \mathrm{CC} 0 \pi$-Np selected sample. The cut on the shower distance is removed to include events where the photon conversion is far from the neutrino interaction vertex. Thus, our final sample will mainly contain NC events, with some contamination of $\nu_{\mu} \mathrm{CC} \pi^{0}$ events where the muon track was tagged as a proton-like track.

Figure 6.57 shows the comparison between data and Monte Carlo for the reconstructed energy spectrum $E_{\text {deposited }}$ of the NC-enhanced event spectrum. The agreement is good both in shape and normalisation: the data points are within the systematic uncertainties of the simulation in every bin.

\section{CC $\nu_{\mu}$-enhanced selection}

It is possible to enhance the presence of the $\mathrm{CC} \nu_{\mu}$ background (defined as beam intrinsic $\nu_{\mu}$ in our analysis) by (1) removing the cut on the total number of hits in the collection plane, (2) removing the cut on the fraction of shower hits, (3) requiring a minimum track length, (4) requiring at least a track with $40<\chi_{p}^{2}<220$ (muon-like track), and (5) requiring that the event is selected by the external $\nu_{\mu}$ CC-inclusive analysis 102 (see Figures 6.34a, 6.36a, 6.40c. Also in this case the 


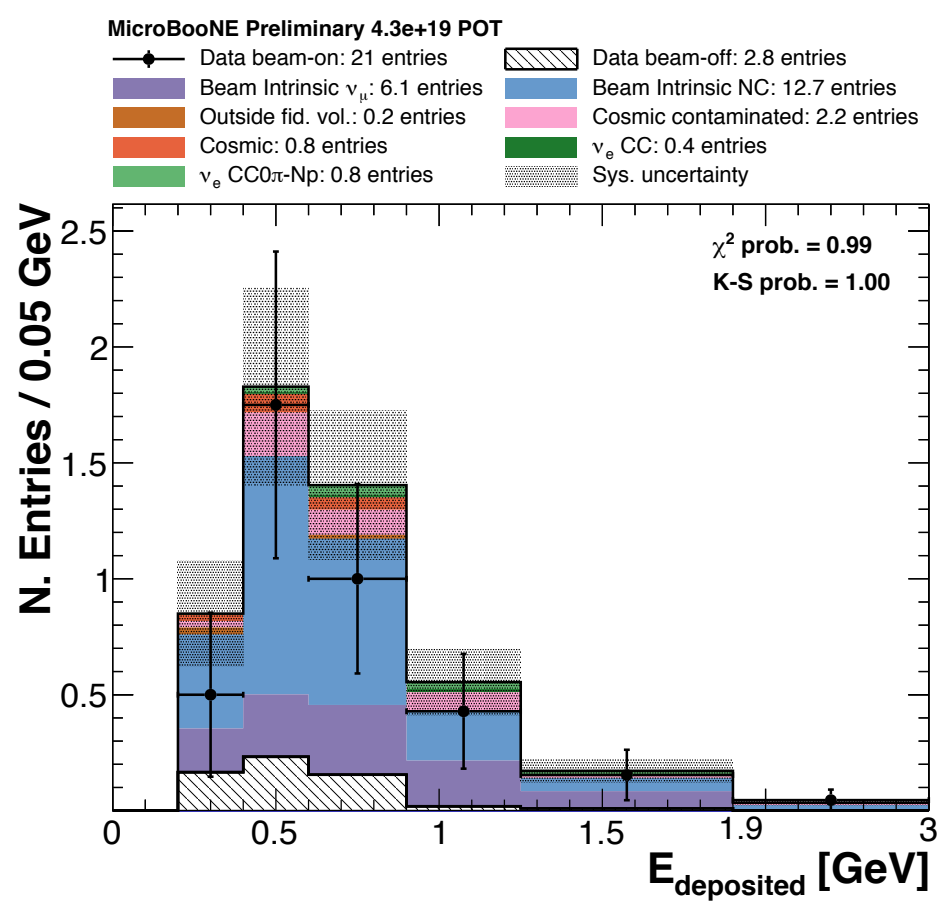

Figure 6.57: Reconstructed energy spectrum of the events selected with the NC-enhanced reverse cuts. The black points represent the data with statistical uncertainties. The coloured stacked histograms represent the simulated events, with the hatched histogram corresponding to the data beam-off sample. The shaded area represents the systematic uncertainty.

$\mathrm{CC} \nu_{\mu}$-enhanced sample will be orthogonal to the $\nu_{e} \mathrm{CC} 0 \pi-\mathrm{Np}$ selected sample. A CC $\nu_{\mu}$ event has, by definition, a muon in the final state: as such, requiring a track length larger than $20 \mathrm{~cm}$ and changing the cut on the proton $\chi_{p}^{2}$ score decreases our muon-rejection power. The goal of the external analysis is to select $\mathrm{CC} \nu_{\mu}$ events, so instead of vetoing those events as described in Section 6.2.8, we invert this requirement by allowing only these events.

Figure 6.58 shows the agreement between data and Monte Carlo for the reconstructed energy spectrum of the $\mathrm{CC} \nu_{\mu}$-enhanced event sample. The agreement is good both in shape and normalisation: the data points are within the systematic uncertainties of the simulation in every bin.

\subsubsection{NuMI beam event studies}

It is possible to run this analysis on the complementary and independent neutrino dataset, acquired with the NuMI beam trigger. The NuMI beam is created from 


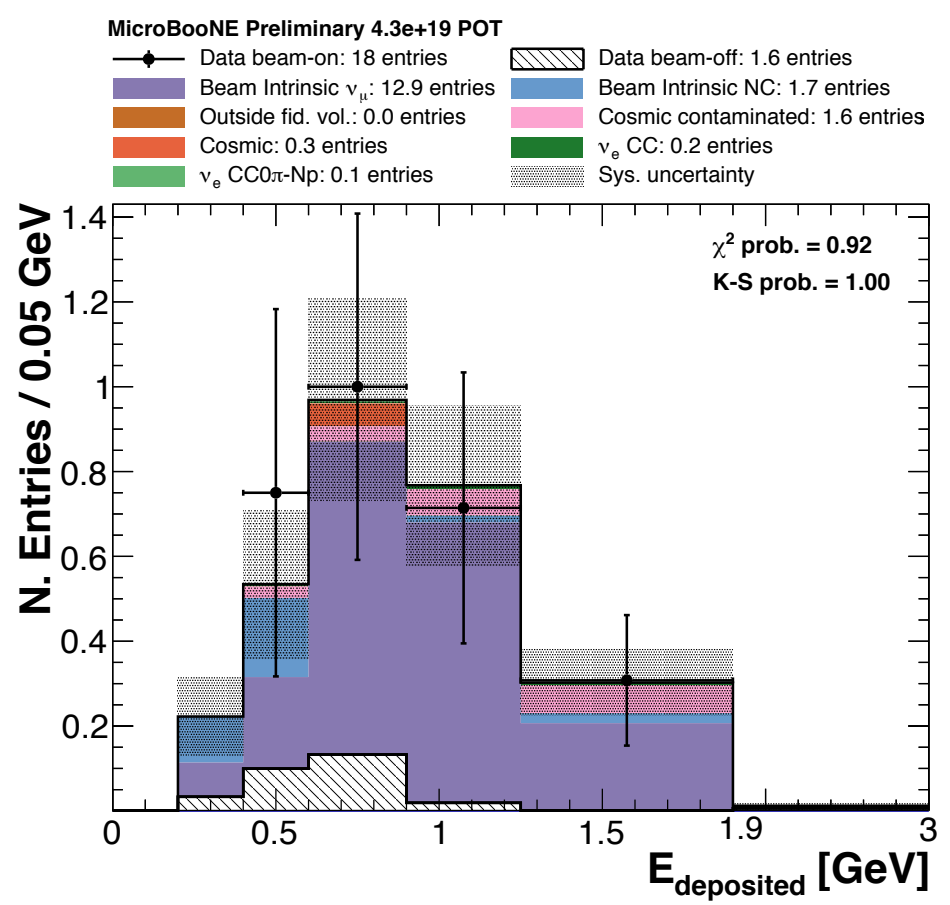

Figure 6.58: Reconstructed energy spectrum of the events selected with the CC $\nu_{\mu^{-}}$ enhanced reverse cuts. The black points represent the data with statistical uncertainties. The coloured stacked histograms represent the simulated events, with the hatched histogram corresponding to the data beam-off sample. The shaded area represents the systematic uncertainty. The bottom part of the plot shows the ratio between the data beam-on events and the stacked histograms.

$120 \mathrm{GeV}$ protons hitting a carbon target 122 , while the BNB is created from 8 $\mathrm{GeV}$ protons on a beryllium target. The NuMI beam has also a higher intrinsic $\nu_{e}$ component than the BNB (5\% vs. $\left.0.5 \%\right)$. Figure 6.59 shows a comparison of the NuMI and BNB beam fluxes for the MicroBooNE detector. Even though it is around $8^{\circ}$ off-axis, MicroBooNE still receives $\sim 2500 \nu_{e}$ interactions per year.

As such, a study of the events selected in the NuMI dataset is of fundamental importance to validate the $\nu_{e} \mathrm{CC} 0 \pi-\mathrm{Np}$ selection algorithm, since it provides a completely independent set of electron neutrinos with different energy and angular distributions. However, the price to pay is the poor understanding of the flux at such off-axis angle. This does not affect the validation, but it makes a search for an excess extremely challenging.

In order to run on this data sample, it is necessary to change the requirement on the reconstructed flash, since the beam-gate window is $[6,16]$ us after the 


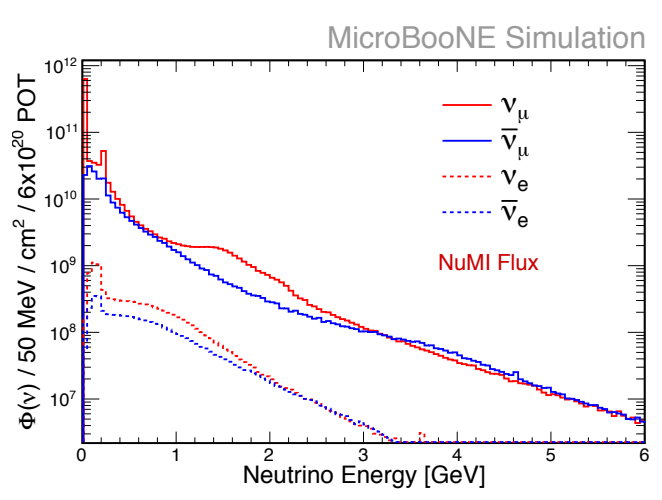

(a) NuMI beam flux.

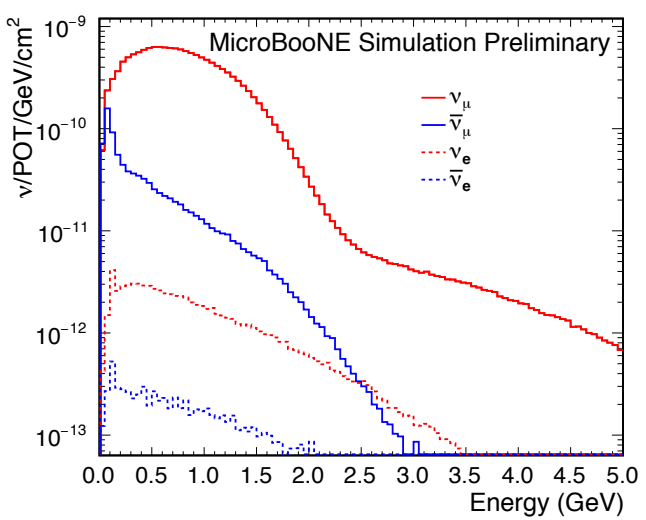

(b) BNB beam flux.

Figure 6.59: NuMI and BNB neutrino fluxes for each neutrino and antineutrino component, when the beams are in neutrino mode.

trigger time. We apply the same rectangular cuts described in Section 6.4.1. plus a threshold of $100 \mathrm{MeV}$ on the reconstructed energy of the leading shower. This last cut allows us to remove a large fraction of cosmogenic background without significantly affecting our $\nu_{e} \mathrm{CC} 0 \pi-\mathrm{Np}$ selection efficiency.

Figure 6.60 shows the number of selected events in data and Monte Carlo for $2.3 \times 10^{20}$ NuMI POT, collected between February and June 2016 in neutrino mode Proper systematic uncertainties for NuMI events still need to be assessed, since the off-axis positioning introduces a large uncertainty in the flux that needs to be carefully evaluated. The overall systematic uncertainty on the number of BNB selected events before any cut is $28.2 \%$, as described in detail in Chapter 7 . A conservative estimate gives an expected $30 \%$ systematic uncertainty in the number of selected NuMI events after the rectangular cuts.

We select 46 data events, $39.7 \pm 11.9$ signal $\left(\nu_{e} \mathrm{CC} 0 \pi\right.$-Np) events, and $23.1 \pm 6.9$ background events. In order to calculate the significance of the detection of $\nu_{e} \mathrm{CC} 0 \pi$ Np events, it is necessary to take into account the large size of the signal $s$ with respect to the background $b$. In this case, the usual formula:

$$
\sigma=\frac{s}{\sqrt{b}}
$$

is not valid. Thus, we adopt a profile-likelihood ratio test, as described in 123 . Assuming a $30 \%$ systematic uncertainty, the expected (observed) significance for 


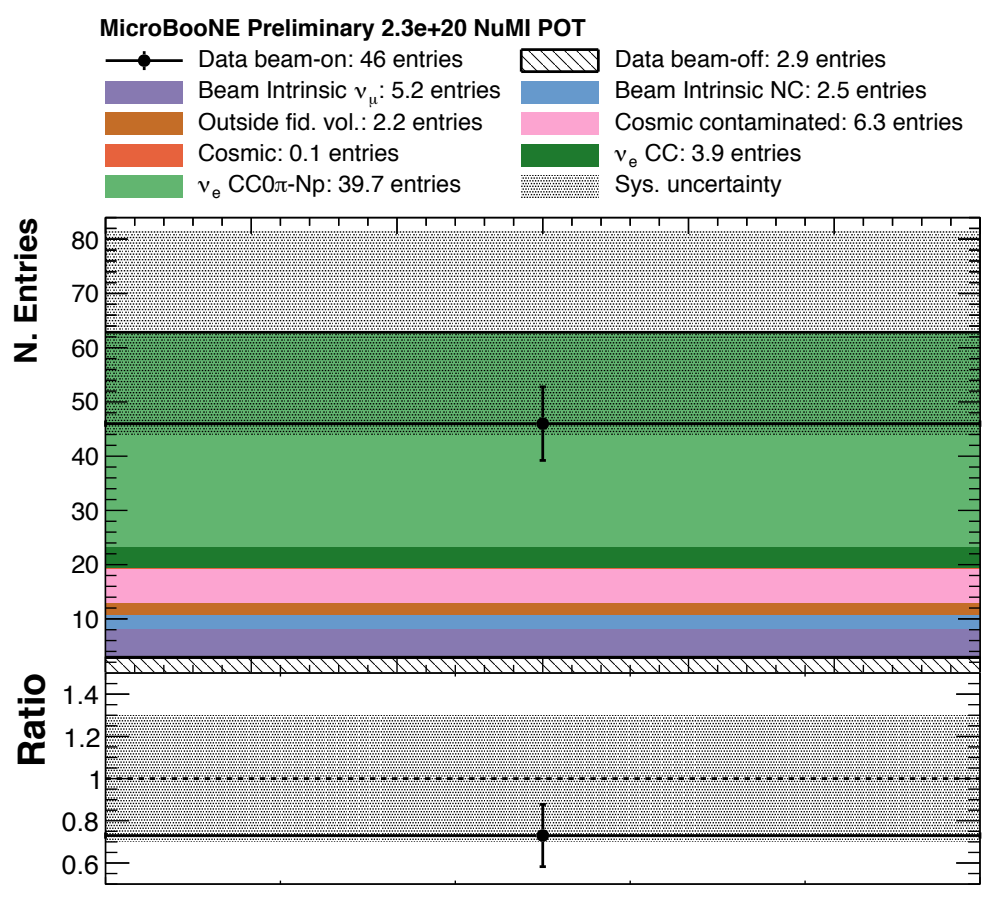

Selected events

Figure 6.60: Number of selected events after the selection and rectangular cuts described in Section 6.4.1, plus an additional threshold on the leading shower energy of $100 \mathrm{MeV}$. The simulated events are classified according to the event category. The number of events corresponds to $2.3 \times 10^{20} \mathrm{NuMI}$ POT in neutrino mode.

the detection of $\nu_{e} \mathrm{CC} 0 \pi$-Np events is $3.4 \sigma(2.2 \sigma)$. The statistical-only significance is $6.8 \sigma(4.2 \sigma)$.

This result represents a very important cross-check for the analysis, since it gives us confidence that we are indeed selecting $\nu_{e} \mathrm{CC} 0 \pi-\mathrm{Np}$ interactions. It also provides a larger sample of electron neutrinos, allowing us to study in detail the shower properties, which will lead to improved cuts and selection efficiencies. 


\section{7}

Systematic uncertainties

\section{Contents}

\begin{tabular}{|c|c|}
\hline 7.1 & Introduction \\
\hline 7.2 & Flux systematic uncertainties \\
\hline 7.3 & Cross-section systematic uncertainties \\
\hline 7.4 & Detector systematic uncertainties. . . \\
\hline 7.5 & Summary . . . . . . . . \\
\hline
\end{tabular}

In this chapter we will describe and evaluate the main systematic uncertainties which affect the selection of $\nu_{e} \mathrm{CC} 0 \pi-\mathrm{Np}$ events and the sensitivity to the lowenergy excess of electron-like events.

\subsection{Introduction}

In this analysis, the systematic uncertainties which affect the measurements of the quantities described in Section 6.2 can be divided into three main categories.

Flux simulation. The amount of $\nu_{\mu}$ and $\nu_{e}$ reaching the MicroBooNE detector is evaluated by an independent simulation of the neutrino flux of the BNB, used also in the MiniBooNE experiment. This simulation is affected by uncertainties which need to be taken into account. 
Cross-section modelling. Our Monte Carlo simulation relies on the neutrino generator provided by the GENIE collaboration [99]. This generator can be configured to use different physics models and interaction parameters (e.g. axial mass in a CCQE interaction, cross-section normalisation factors, etc.), which can affect the relative abundance and the energy of the particles in the final state. The parameters and the physics models are described in detail in the GENIE Physics Manual 124.

Detector simulation. The detector response (noise removal, signal processing, hit reconstruction) must be carefully simulated in order to achieve a good agreement between data and Monte Carlo in the reconstructed quantities used for background rejection. Several detector parameters are not perfectly understood and the effect of these uncertainties must be assessed.

The systematic uncertainties related to the flux and the neutrino generator are evaluated by simulating several universes, where the GENIE and flux parameters are varied within their uncertainties. If the parameter $p$ is known with uncertainty $\Delta p$, then the single universe will have the parameter varied by $c \Delta p$, where $c$ is randomly drawn from a Gaussian distribution with $\mu=0$ and $\sigma=1$.

The covariance matrix for the flux and cross-section uncertainties is defined as:

$$
E_{i j}^{\mathrm{flux}, \mathrm{GENIE}}=\frac{1}{N_{u}} \sum_{u=0}^{N_{u}}\left(x_{i}^{u}-x_{i}^{c v}\right)\left(x_{j}^{u}-x_{j}^{c v}\right),
$$

where $i, j$ are the bins of the $x$ reconstructed quantity, $N_{u}$ is the number of simulated universes (100 in our case), $x^{u}$ is the reconstructed quantity in the universe $u$ and $x^{c v}$ is the central value of the reconstructed quantity. The detector systematic uncertainties are instead evaluated by varying one parameter at the time. In this case, the covariance matrix is:

$$
E_{i j}^{\mathrm{det}}=\sum_{s=0}^{v}\left(x_{i}^{s}-x_{i}^{c v}\right)\left(x_{j}^{s}-x_{j}^{c v}\right),
$$

where $v$ is the number of detector variations samples and $x^{s}$ is the value of the reconstructed variable in the sample $s$. The total covariance matrix $E$ is defined as:

$$
E^{\mathrm{sys}}=E^{\mathrm{stat}}+E^{\mathrm{flux}}+E^{\mathrm{GENIE}}+E^{\mathrm{det}}
$$


where $E^{\text {stat }}$ corresponds to the statistical uncertainty of the Monte Carlo and data beam-off samples, given their limited size. The systematic uncertainty for the bin $i$, shown in the plots of Section 6.2, corresponds to square root of the diagonal elements of the covariance matrix $\sqrt{E_{i i}}$. The fractional covariance matrix $F$ is directly obtained from the covariance matrix and the central values as:

$$
F_{i j}=\frac{E_{i j}}{x_{i}^{c v} x_{j}^{c v}}
$$

The linear correlation matrix $\rho$ is defined as:

$$
\rho_{i j}=\frac{E_{i j}}{\sqrt{E_{i i} E_{j j}}} .
$$

This matrix provides a measure of the correlation of the uncertainty between the bin $i$ and the bin $j$. The value of $\rho_{i j}$ can be between -1 (completely anti-correlated) and +1 (completely correlated). The elements on the diagonal will have by definition $\rho_{i i}=+1$. Positive correlation is usually caused by effects which change the overall number of events (e.g. the magnitude of the neutrino flux): in this case the increase of events in the bin $i$ will correspond to an increase of events in the bin $j$ (and viceversa). Negative correlation is instead caused by effects which change the shape of the distribution and keep the number of events constant: in this case the increase of events in the bin $i$ will correspond to a decrease of events in the bin $j$ (and vice-versa).

\subsection{Flux systematic uncertainties}

MicroBooNE is using the same simulation of the BNB flux developed by the MiniBooNE collaboration. The different contributions to the systematic uncertainties of this simulation are thoroughly described in 46 and summarised in Section 4.3.2.

In this analysis, the systematic uncertainties related to the flux simulation are evaluated by generating 100 universes, where the flux parameters are varied within their uncertainties and their correlation is taken into account.

Figure $7.2 \mathrm{a}$ shows the central value of the $\nu_{e} \mathrm{CC} 0 \pi$-Np selection efficiency and the corresponding value for each flux variation universe. Also here, the variation in the efficiency is small, as expected. Figure $7.2 \mathrm{~b}$ shows a bias of the variation 
samples with respect to the nominal simulation, which does not correspond to the average of the universes. This is caused by an inconsistency of the pion production cross-section used in the generation of the universes. Figure 7.1 shows the pion production cross-section as measured by HARP 125 and the fit with the SanfordWang parametrisation [126, which are not in agreement at low momentum. The nominal Monte Carlo simulation uses the result of the Sanford-Wang fit, while the systematic uncertainties are evaluated with a spline interpolation of the HARP data.

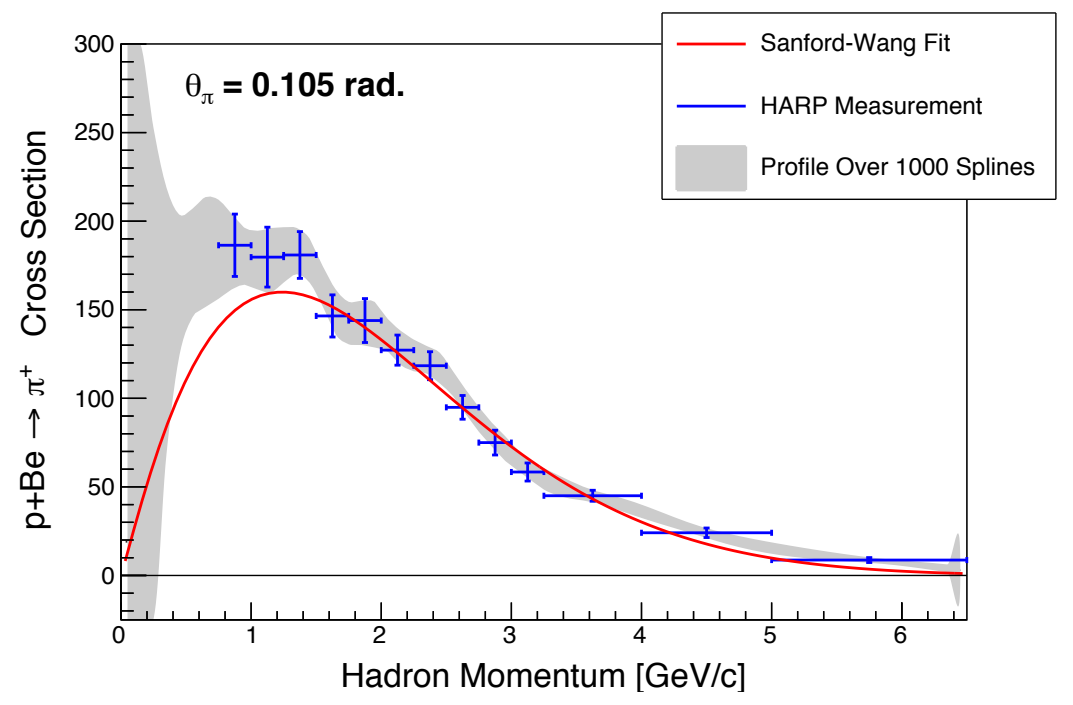

Figure 7.1: Pion production at a fixed angle as measured by the HARP experiment and fit with the Sanford-Wang parametrisation.

In this case, the nominal value is considered as the central value in the covariance matrix definition of Equation 7.1. The flux-related uncertainty in the number of simulated selected events (no beam-off data) before the background rejection is $12.3 \%$.

Also in this case the correlation matrix in Figure $7.2 \mathrm{~d}$ shows that the flux systematic effect are positively correlated in the $E_{\text {deposited }}$ bins, which means they generally increase of decrease the total number of neutrino interactions. 


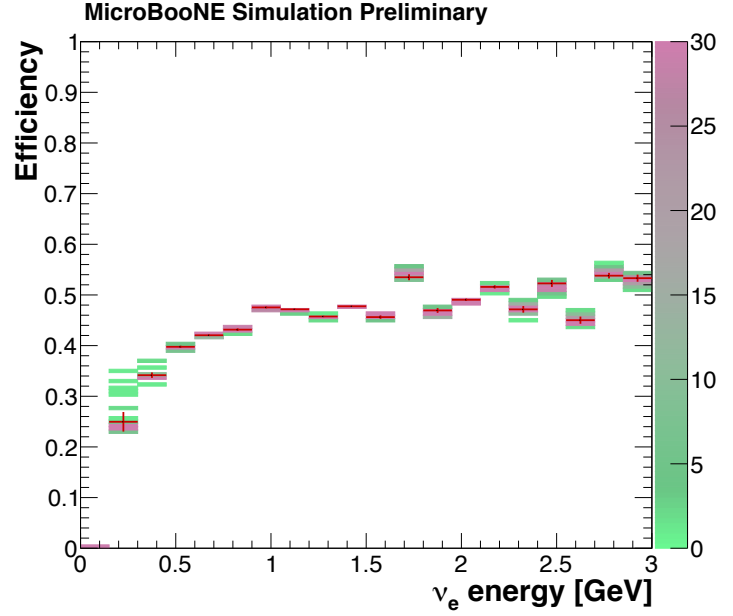

(a) $\nu_{e} \mathrm{CC} 0 \pi-\mathrm{Np}$ selection efficiency.

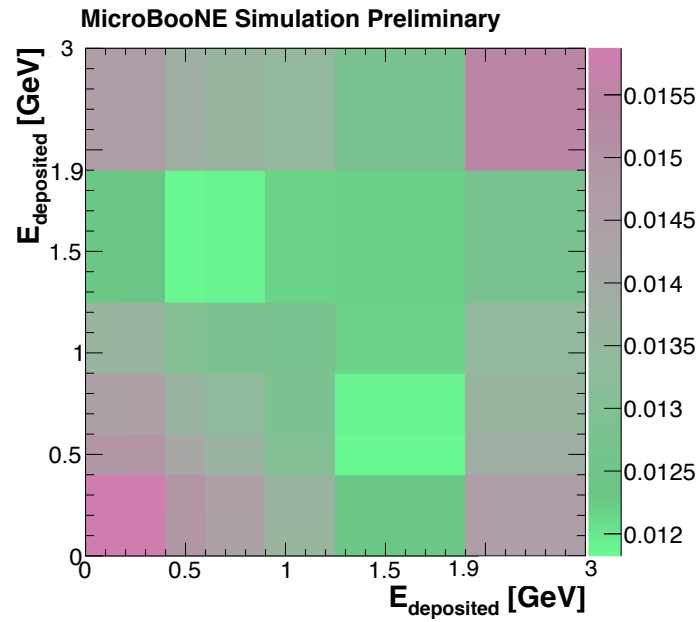

(c) Fractional covariance matrix.

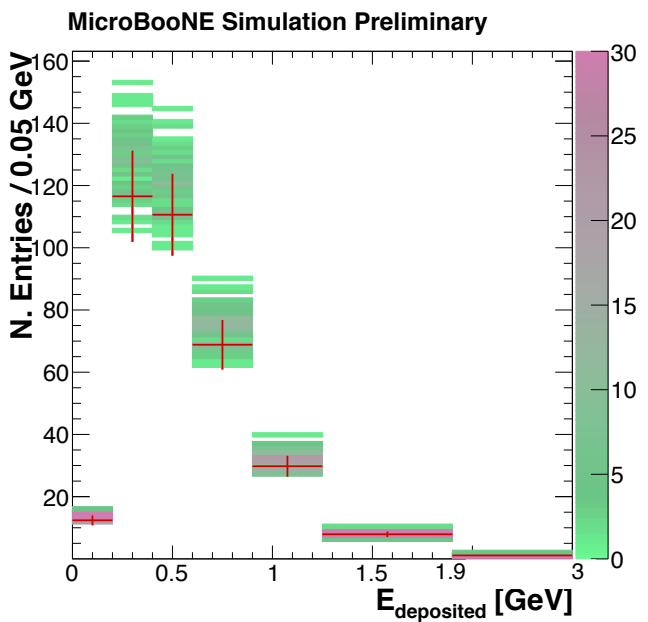

(b) Energy spectrum of the selected events.

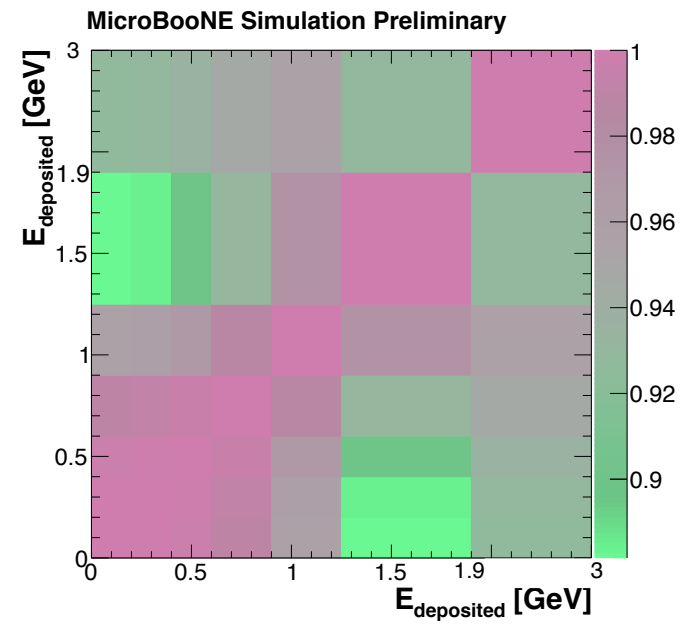

(d) Correlation matrix.

Figure 7.2: Selection efficiency, reconstructed energy spectrum, fractional covariance matrix, and correlation matrix obtained by varying the BNB flux parameters in 100 simulated universes. The colour scale for the selection efficiency and the energy spectrum corresponds to the number of universes. The red bars correspond to the central value and its flux systematic uncertainty only. The data beam-off sample is not included in these plots. 


\subsection{Cross-section systematic uncertainties}

In order to estimate the cross-section systematic uncertainties, the standard GENIE parameters described in the Chapter 9 of the GENIE User Manual 124 are simultaneously varied within their uncertainties in $N_{u}=100$ simulated universes. The correlation between the parameters is accounted for internally by GENIE. A calculated weight gets assigned to each universe, which is applied when filling the histograms of the reconstructed quantities. The uncertainties shown here do not include systematic effects associated with Random Phase Approximation (RPA) and with Meson Exchange Current (MEC) interactions. RPA refers to long-range multi-nucleon correlations which suppress the neutrino-nucleon cross-section at low-exchanged momentum $Q^{2}$ 127]. MEC interactions involve the scatter between the neutrino and a correlated pair of nucleons (2p-2h) [38]. These two systematic effects will be quantified in a future version of the analysis.

Figure $7.3 \mathrm{a}$ shows the central value of the $\nu_{e} \mathrm{CC} 0 \pi$-Np selection efficiency and the corresponding value for each GENIE variation universe. The variation in this case is expected to be small, since we are essentially dividing two distributions (passed and total) with similar weights. Figure $7.3 \mathrm{~b}$ and Figure $7.3 \mathrm{c}$ show that the variations in the reconstructed energy spectrum are larger at lower energies, which is the energy region most affected by the GENIE parameters uncertainties. The GENIE-related uncertainty in the number of simulated selected events (no beam-off data) before the background rejection is $7.9 \%$. The correlation matrix in Figure $7.3 \mathrm{~d}$ shows that the bins are mostly positively correlated. This means that the variations of the GENIE parameters have in our case mostly a normalisation effect (i.e. they change the total number of events).

\subsection{Detector systematic uncertainties}

The detector systematic uncertainties have been measured by simulating several samples where a single detector parameter is varied by its estimated $\pm 1 \sigma$ uncertainty 

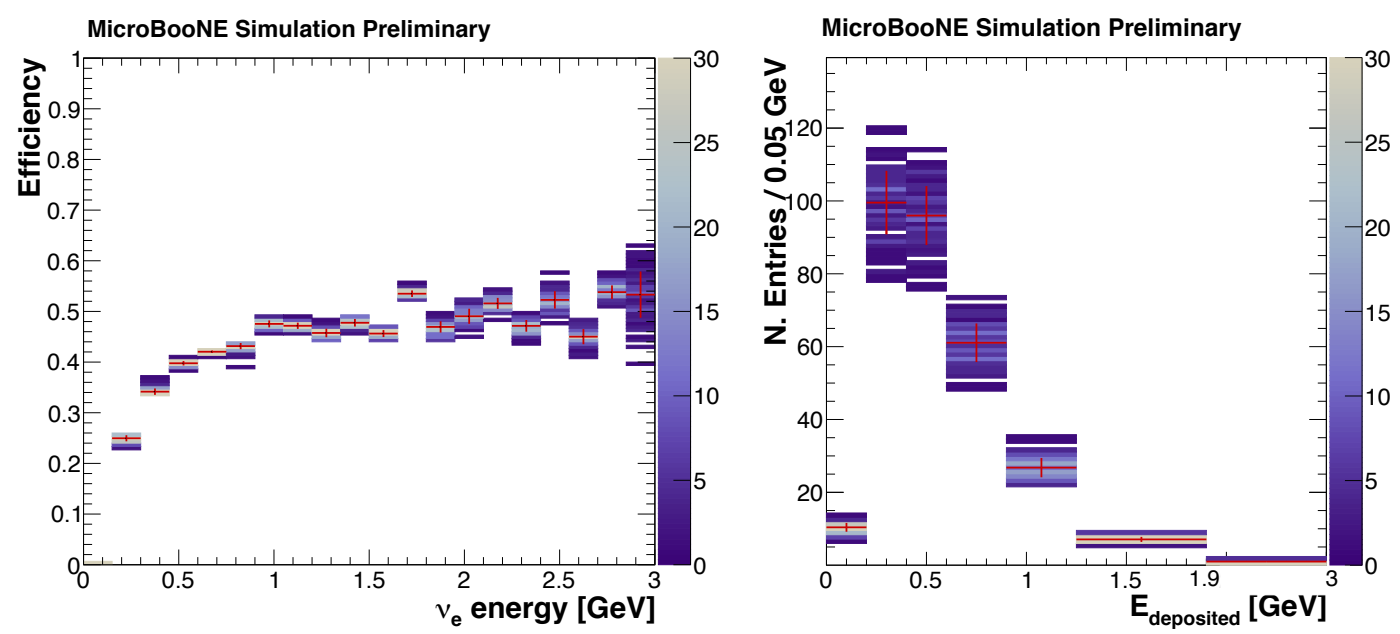

(a) $\nu_{e} \mathrm{CC} 0 \pi$-Np selection efficiency.

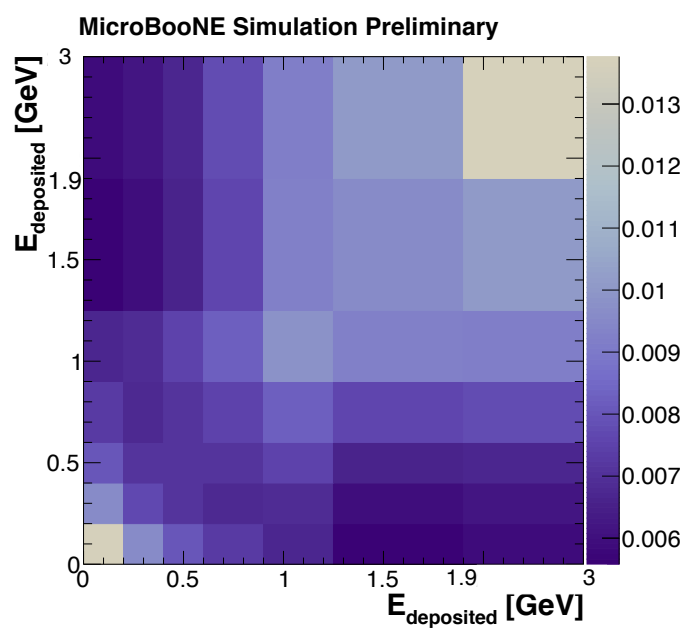

(c) Fractional covariance matrix. (b) Energy spectrum of the selected events.

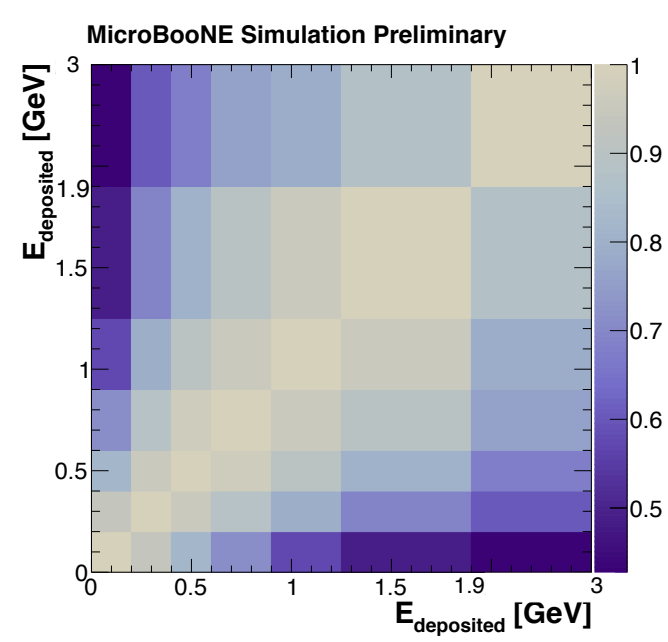

(d) Correlation matrix.

Figure 7.3: Selection efficiency, reconstructed energy spectrum, fractional covariance matrix, and correlation matrix obtained by varying the standard GENIE parameters in 100 simulated universes. The colour scale for the selection efficiency and the energy spectrum corresponds to the number of universes. The red bars correspond to the central value and its cross-section systematic uncertainty only. Events with MEC interactions are not included in these plots and the uncertainties do not include RPA effects. The data beam-off sample is not included in these plots. 
or where a different physics model is used. The detector variations taken into consideration are described below.

Space-charge effect. The $x$-dependence of the space-charge effect is estimated with a data-driven procedure and its magnitude is scaled by 0.7 .

Dynamic Induced Charge. Improved simulation of the induction of charge on the neighbouring wires.

Light simulation. Improved simulation of the light production in the detector.

Saturated channels. Channels that tend to saturate are turned off in the simulation.

Misconfigured channels. Channels with misconfigured ASIC gains and shaping are turned off in the simulation.

Electron lifetime. Lifetime of the electron in the detector is reduced to $10 \mathrm{~ms}$, which corresponds to a lower LAr purity.

Recombination model. The Birks model of recombination 80 is used instead of the modified box model 108 .

Longitudinal diffusion. The longitude diffusion is varied by $\pm 1 \sigma$ of its estimated uncertainty.

Transverse diffusion. The transverse diffusion is varied by $\pm 1 \sigma$ of its estimated uncertainty.

Wire noise. The amount of noise on the wires is varied by $\pm 1 \sigma$ of its estimated uncertainty.

PE noise. The amount of single-PE noise in the PMTs is varied by $\pm 1 \sigma$ of its estimated uncertainty.

Cryostat light. The light outside the TPC but inside the cryostat is increased by $20 \%$. 
Wire response. The wire response functions are squeezed by $20 \%$.

In this case, the covariance matrix is calculated using the definition in Equation 7.2. The fractional covariance matrix and the reconstructed energy spectrum are shown in Figure 7.4b and Figure 7.4a, respectively. The uncertainty related to the detector systematic effects in the number of simulated selected events is $24.0 \%$. The limited size of the detector variation samples does not allow us to calculate the covariance matrix after the rectangular or BDTs cuts. For this reason, a flat $24.0 \%$ detector uncertainty is applied to the simulated events not rejected by the rectangular or BDTs cuts. These high detector systematic uncertainties reflect our knowledge of the detector when the samples were generated (August 2018). However, we are now able to simulate the detector more precisely, by including e.g. a full data-driven map of the space-charge effect and the charge induction on neighbouring wires, which will lead us to significantly smaller systematic uncertainties.

The details of the detector systematic uncertainties are listed in Table 7.1. In this case, the detector variations $\sigma_{\text {det }}$ are defined as:

$$
\sigma_{\mathrm{det}}=\frac{x^{c v}-x^{s}}{x^{c v}}
$$

where $x^{c v}$ is the number of the selected events in the central value sample and $x^{s}$ is the number of selected events in the variation sample $s$. For the samples where one detector parameter was varied by its $\pm 1 \sigma$ uncertainty, the larger variation is quoted.

The components showing the largest variations are the Cosmic, Cosmic contaminated, and Outside fid. vol. background categories. In particular, the sample with a different simulation of the space-charge effect causes a variation of the Cosmic, Cosmic contaminated, and Outside fid. vol. components of $28.0 \%, 42.0 \%$, and $32.5 \%$, respectively. This is expected, since these events are mostly located near the borders of the TPC, so if the magnitude of the space-charge effect is decreased, more events will be shifted towards the borders and then removed by the fiducial volume cut.

The sample with an improved simulation of the charge induced on the neighbouring wires introduces a large variation in the Beam intrinsic $\nu_{\mu}$ component $(19.0 \%$ more events in the detector variation sample). This is because this simulation 
makes ionisation tracks look more like shower objects, increasing the number of events which satisfy our topology requirement.

The correlation matrix in Figure $7.4 \mathrm{c}$ shows a positive correlation among the $E_{\text {deposited }}$ bins, mainly dominated by the space-charge effect. As explained above, an increase (decrease) in the magnitude of this effect cause an increase (decrease) in the number of cosmogenic background events selected.

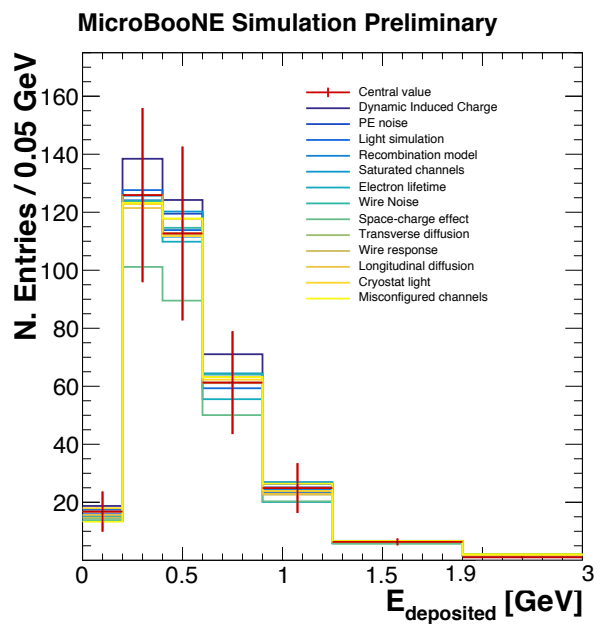

(a) Energy spectrum of selected events.

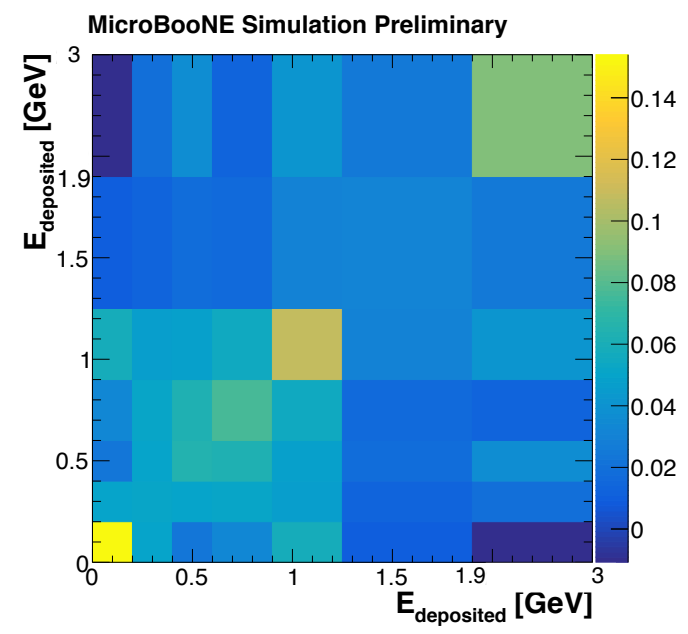

(b) Fractional covariance matrix.

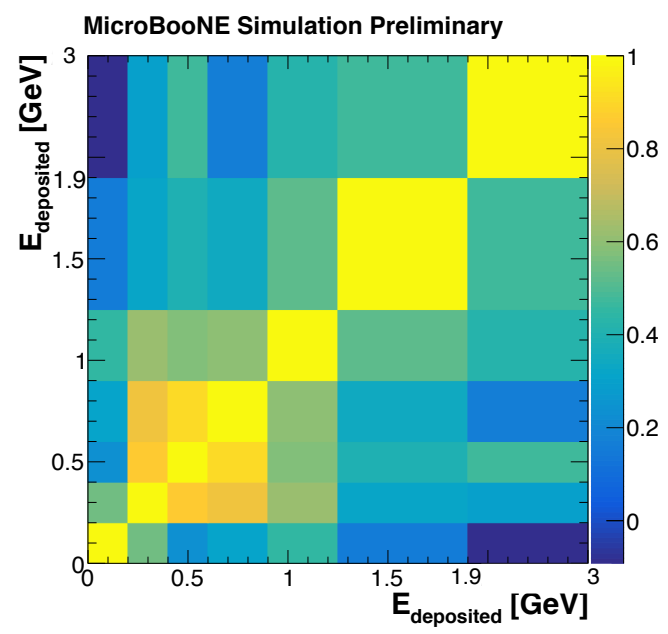

(c) Correlation matrix.

Figure 7.4: Reconstructed energy spectrum, fractional covariance matrix, and correlation matrix obtained with the detector variations samples. The red bars correspond to the central value and its detector systematic uncertainty only. The data beam-off sample is not included in these plots. 
Table 7.1: Summary of the detector uncertainties variations in the BNB+cosmic sample, broken down by event category.

\begin{tabular}{lrrrrrr}
\hline Sample & $\begin{array}{r}\text { Beam } \\
\text { intrinsic } \\
\nu_{\mu}[\%]\end{array}$ & $\begin{array}{r}\text { Beam } \\
\text { intrinsic } \\
\text { NC [\%] }\end{array}$ & $\begin{array}{r}\text { Outside } \\
\text { fid. vol. } \\
{[\%]}\end{array}$ & $\begin{array}{r}\text { Cosmic } \\
\text { contam. } \\
{[\%]}\end{array}$ & $\begin{array}{r}\text { Cosmic } \\
{[\%]}\end{array}$ & $\begin{array}{r}\text { Total } \\
{[\%]}\end{array}$ \\
\hline SCE & 8.5 & 2.9 & 32.5 & 42.0 & 28.0 & 20.3 \\
Reco. model & 3.2 & 4.9 & 14.4 & 4.0 & -3.5 & 2.5 \\
DIC & -19.0 & 2.8 & 15.9 & -7.3 & -11.5 & -8.8 \\
Light sim. & 3.6 & 0.4 & 7.0 & 20.1 & -4.9 & 4.5 \\
Sat. chan. & -6.9 & 3.6 & 4.2 & -0.4 & -5.5 & -1.4 \\
$e^{-}$lifetime & 9.1 & 5.2 & 21.1 & 4.0 & 6.3 & 7.0 \\
Long. diff. & 3.2 & 0.2 & 12.0 & 11.9 & -4.3 & -0.4 \\
Trans. diff. & 1.5 & 2.2 & 5.0 & 3.9 & -3.1 & 1.1 \\
Mis. chan. & -4.6 & 4.0 & 4.2 & 0.5 & -2.4 & -0.8 \\
Wire noise & 3.4 & 3.7 & 6.0 & 5.9 & -3.0 & 0.5 \\
PE noise & -0.7 & 2.4 & 14.4 & 5.1 & -8.6 & -0.2 \\
Cryo. light & 2.9 & 2.1 & 14.3 & 3.5 & -2.9 & 1.1 \\
Wire res. & 3.9 & 4.3 & 5.0 & 3.7 & -1.1 & 2.7 \\
\hline
\end{tabular}

\subsection{Summary}

Table 7.2 shows the uncertainty in the number of selected events before the background rejection for the cross-section, flux, and detector systematic variations, broken down by event category. There are no detector variations available for the $\nu_{e}+$ cosmic and dirt samples. As such, at this stage, the uncertainty on these samples is assumed to be the same one of the BNB+cosmic sample.

The total uncertainty on the number of selected events before the background rejection stage, obtained with Equation 7.3 , is $28.2 \%$. The statistical uncertainty on the data off-beam sample is $1.0 \%$.

The flux and cross-section systematic uncertainties do not show a large variation among the background categories of the BNB+cosmic sample. The smallest cross-section variation is $8.6 \%$ for the Cosmic contaminated and Outside fid. vol. component, while the largest is $10.4 \%$ for the Beam intrinsic $\nu_{\mu}$ category. The flux variations span from $9.3 \%$ for the Cosmic events to $12.7 \%$ for the Beam intrinsic NC component. The flux and cross-section variations for the Cosmic and the Cosmic 
Table 7.2: Summary of the systematic uncertainties variations in the BNB+cosmic sample, break down by event category.

\begin{tabular}{lrrr}
\hline Category & Cross-section [\%] & Flux [\%] & Detector [\%] \\
\hline$\nu_{e}$ CC0 $\pi$-Np & 16.8 & 13.0 & - \\
$\nu_{e}$ CC & 11.1 & 10.9 & - \\
\hline Beam intrinsic $\nu_{\mu}$ & 10.4 & 12.1 & 25.6 \\
Beam intrinsic NC & 9.5 & 12.7 & 11.9 \\
Outside fid. vol. & 8.6 & 11.0 & 51.9 \\
Cosmic & 9.3 & 9.3 & 34.0 \\
Cosmic contaminated & 8.6 & 11.0 & 49.5 \\
\hline Total & 7.9 & 12.3 & 24.0 \\
\hline
\end{tabular}

contaminated categories refer to the simulated neutrino interaction in the event, as the cosmic themselves are obviously not affected by the neutrino cross section.

The flux and cross-section uncertainties have been evaluated also for the events in the $\nu_{e}+$ cosmic and dirt samples. The flux (cross-section) uncertainties are $13.0 \%$ and $10.9 \%$ (16.8\% and $11.1 \%$ ) for the $\nu_{e} \mathrm{CC} 0 \pi-\mathrm{Np}$ and the $\nu_{e} \mathrm{CC}$ events, respectively. This higher uncertainties, compared with the $\mathrm{BNB}+$ cosmic sample, reflect the limited $\nu_{e}$ cross-section measurements available.

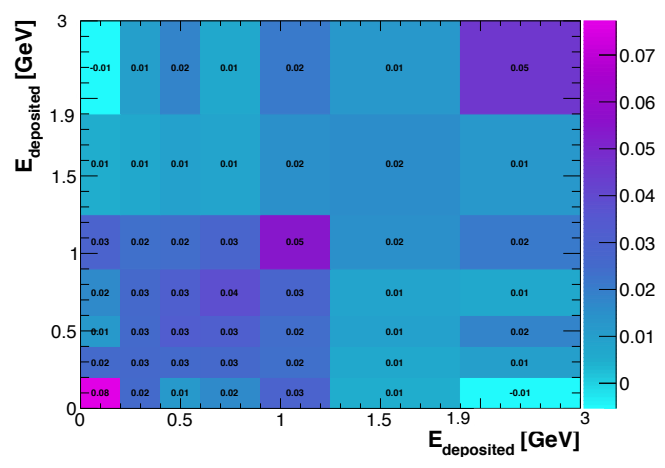

(a) Fractional covariance matrix.

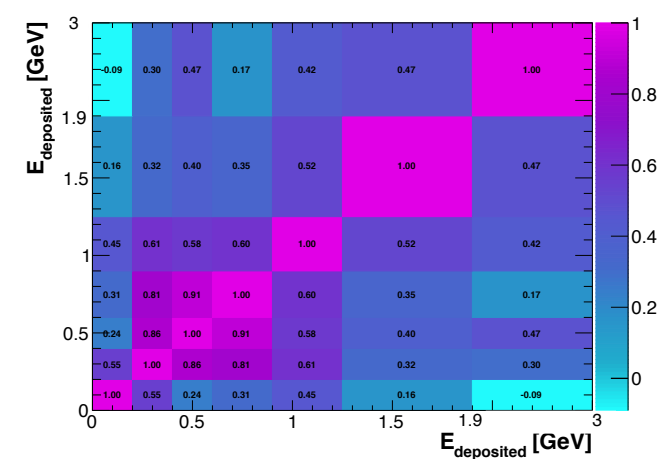

(b) Correlation matrix.

Figure 7.5: Full fractional covariance (left) and correlation (right) matrices obtained by combining the statistical, cross-section, flux, and detector uncertainties for the $E_{\text {deposited }}$ distribution before the background rejection.

Figure 7.5 shows the full fractional covariance and correlation matrices obtained by combining the statistical, cross-section, flux, and detector uncertainties for the 
$E_{\text {deposited }}$ distribution before the background rejection. The covariance matrix is used to calculate the systematic uncertainties shown in Figure 6.33. 


\section{Sensitivity to the MiniBooNE low-energy}

excess

\section{Contents}

\begin{tabular}{|c|c|}
\hline 8.1 & Estimation of the MiniBooNE signal in MicroBooNE \\
\hline 8.2 & Sensitivity to the excess $\ldots \ldots \ldots \ldots \ldots \ldots$ \\
\hline 8.3 & Future improvements . . . . . \\
\hline 8.4 & Improvements and sensitivity \\
\hline
\end{tabular}

In this chapter we will evaluate the current sensitivity of the $\nu_{e} \mathrm{CC} 0 \pi-\mathrm{Np}$ selection to the MiniBooNE low-energy excess in the electron hypothesis. First, the process to estimate the MiniBooNE excess in the MicroBooNE experiment will be described. Then, the sensitivity of the selection will be calculated, taking into account the systematic uncertainties. Thus, the improvements in the selection efficiency and background rejection needed to reach $5 \sigma$ sensitivity will be calculated.

\subsection{Estimation of the MiniBooNE signal in Mi- croBooNE}

In order to assess the sensitivity of our analysis to the MiniBooNE low-energy excess in the electron hypothesis, it is necessary to remove the effects of the MiniBooNE detector response, event reconstruction, and selection from the estimation of the 
excess. This procedure is usually defined as unfolding 128. Figure 8.1 shows the MiniBooNE low-energy excess result used in this section: it includes the $6.46 \times 10^{20}$ POT collected in neutrino mode and analysed in 129 (does not include the latest data run used for the result in 48 ).

\section{MiniBooNE excess, Electron-Like Model}

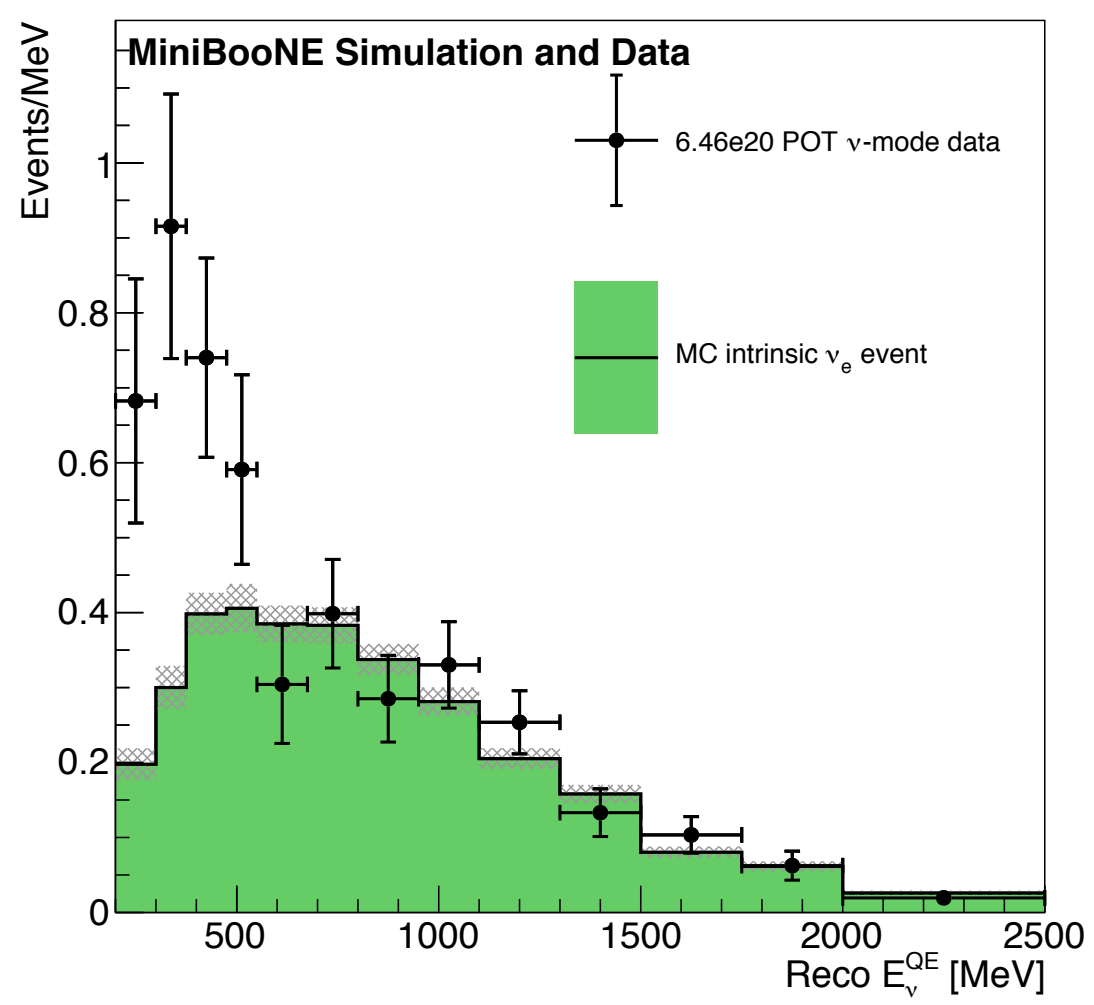

Figure 8.1: The MiniBooNE low-energy excess compared to the simulated MiniBooNE beam-intrinsic $\nu_{e}$ component. The error bars correspond to the data statistical uncertainties on observed data, and the shaded region corresponds to the Monte Carlo systematic uncertainties. From 128 .

The detector and selection effects are entirely described by a response matrix $C$, which transforms a true spectrum $t$, in our case of the true neutrino energy $E_{\nu}$, into a reconstructed spectrum $r$, which for us will be the reconstructed CCQE energy $E_{\nu}^{C C Q E}$ :

$$
t_{i}=C_{i j} r_{j}
$$

Figure 8.2 shows the response matrix $C$ for the MiniBooNE experiment which 
maps $E_{\nu}$ into $E_{\nu}^{C C Q E}$. In order to calculate this matrix, the MiniBooNE collaboration provided us full access to their Monte Carlo simulation and selection code.

\section{MiniBooNE Response Matrix, Electron-like Model}

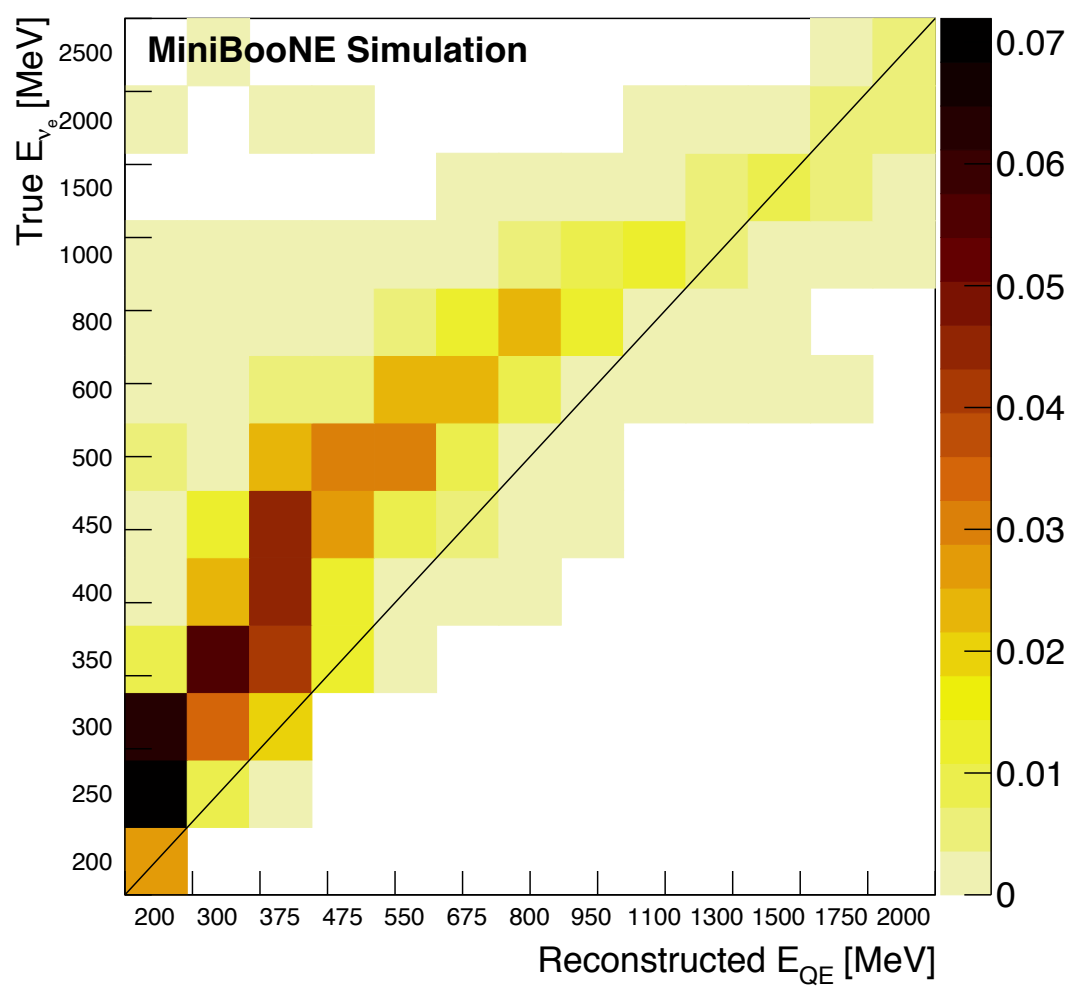

Figure 8.2: The MiniBooNE response matrix for the electron hypothesis of the low-energy excess. From 128.

Technically, the unfolding process consists in the creation of the inverse map $C^{-1}$ from the reconstructed variable $r$, in the MiniBooNE analysis, to the true variable $t$. We decided to use the same energy range of the MiniBooNE collaboration, so we are not looking at the MiniBooNE data and simulation below $200 \mathrm{MeV}$ to perform the unfolding.

However, the folding process (from true to reconstructed variables) usually causes partial loss of information and makes the unfolding procedure not straightforward. In particular, it is possible to have several true distributions corresponding to a single reconstructed distribution. This effect introduces a large uncertainty in our procedure, which can be reduced with the regularisation process. The regularisation 
introduces a small bias (usually motivated by physics arguments) in order to reduce the variance of our possible true distributions.

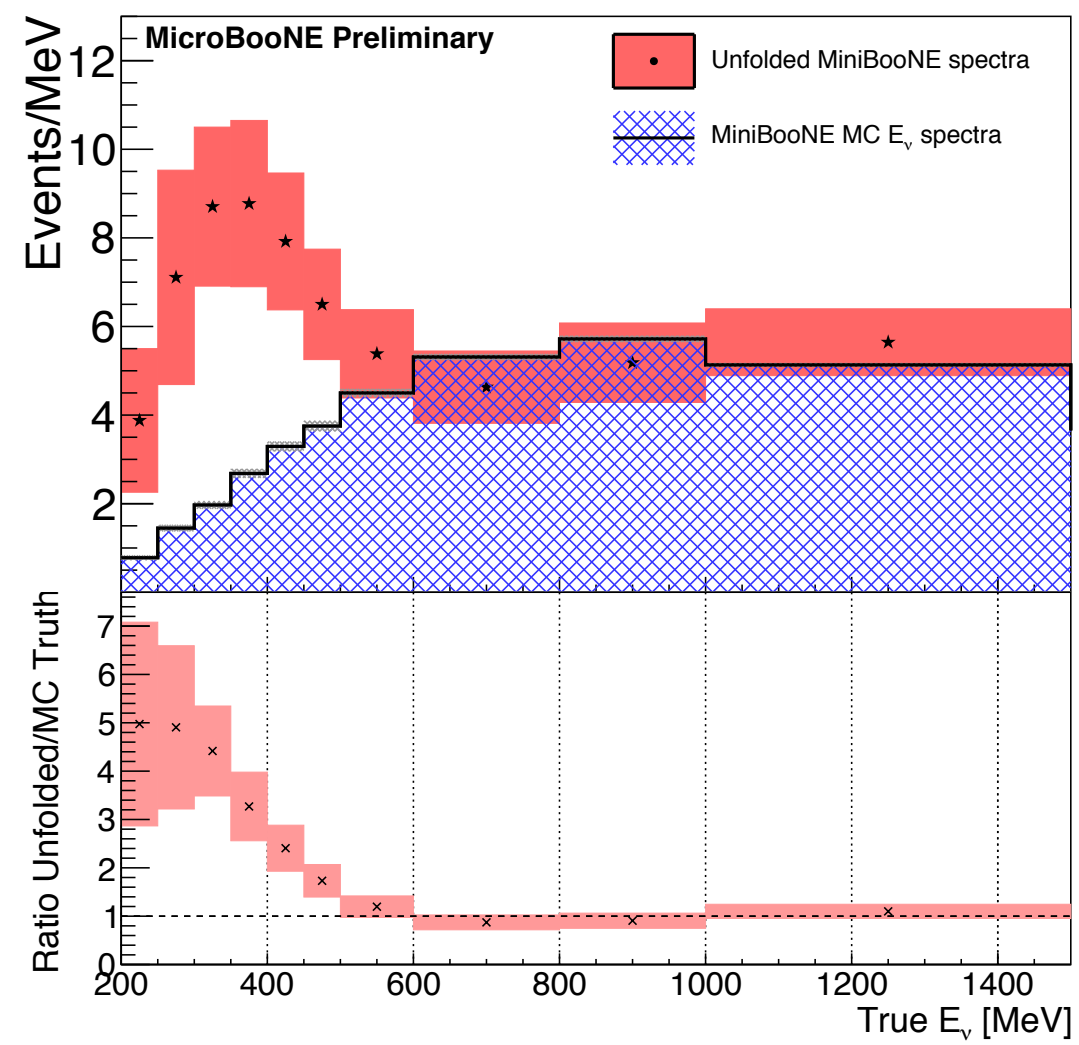

Figure 8.3: Unfolded MiniBooNE beam intrinsic $\nu_{e}$ Monte Carlo and data spectra in the electron hypothesis. It represents the result of the unfolding of Figure 8.1. The filled area correspond to the data unfolding uncertainty. From [128].

The result of the unfolding of the distributions in Figure 8.1 is shown in Figure 8.3. in the electron hypothesis, the MiniBooNE low-energy excess corresponds to a scaling of the beam intrinsic $\nu_{e}$ component as a function of the true $E_{\nu}$ energy. The ratio between the unfolded data spectrum and the unfolded Monte Carlo spectrum is slightly lower than 1 at $600 \mathrm{MeV}<E_{\nu}<1000 \mathrm{MeV}$. In this case, we do not scale our beam intrinsic $\nu_{e}$ component.

\subsection{Sensitivity to the excess}

Once we have unfolded the MiniBooNE low-energy excess, it is possible to process a sample of beam intrinsic $\nu_{e}$, scaled by the factors shown in Figure 8.3, through 
the entire MicroBooNE simulation and reconstruction chain. Figure 8.4 shows the reconstructed energy spectra $E_{\text {deposited }}$ after the application of the rectangular cuts (left) and of the BDTs cuts (right), including the simulated low-energy excess signal (in light green). We select 0.56 (0.40) low-energy excess events after the application of the rectangular cuts (BDTs cuts) for an exposure of the MicroBooNE detector of $4.34 \times 10^{19}$ POT. When scaled to the expected amount of collected POT $\left(13.2 \times 10^{20}\right)$, the selected events become $17.3(12.3)$.

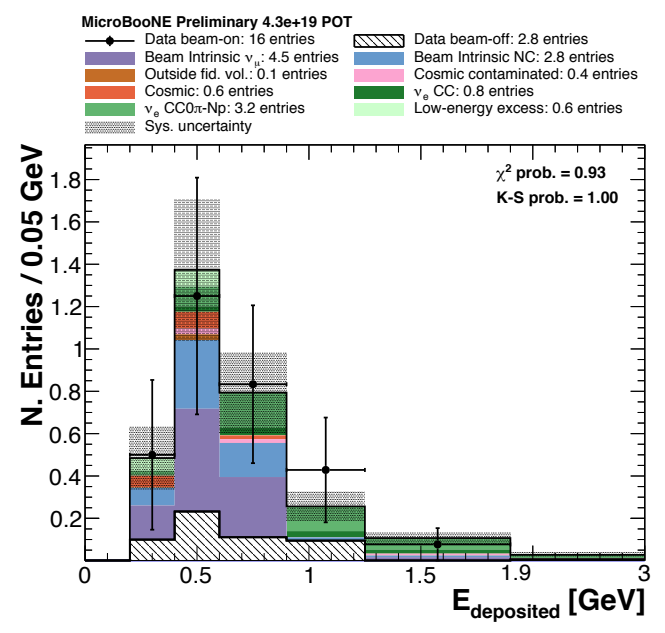

(a) Rectangular cuts.

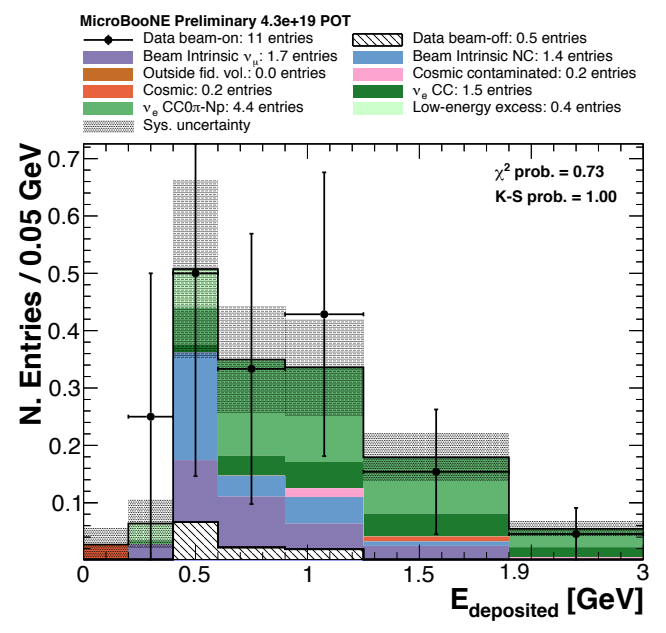

(b) BDTs cuts.

Figure 8.4: Energy spectrum $E_{\text {deposited }}$ of the selected events stacked with the MiniBooNE low-energy excess signal in the electron hypothesis (light green). The black points represent the data with statistical uncertainties. The coloured stacked histograms represent the simulated events, with the hatched histogram corresponding to the data beam-off sample. The shaded area represents the systematic uncertainty. These distributions are identical to the ones in Figure 6.45 and Figure 6.53, except for the low-energy excess signal component.

Table 8.1 shows the expected sensitivity to the MiniBooNE low-energy excess for $13.2 \times 10^{20}$ POT collected by MicroBooNE. The sensitivity has been measured with a $\Delta \chi^{2}$ test 130 as:

$$
\sigma=\sqrt{\Delta \chi^{2}}=\sqrt{\vec{S}^{T} E^{-1} \vec{S}}
$$

where $\vec{S}$ is a vector of length $n$ containing the number of signal events in the $n$ bins and $E$ is the covariance matrix as defined in Equation 7.1. In the case 
of statistical-only significance, the covariance matrix has only diagonal entries, corresponding to the number of background events.

Table 8.1: Summary of the sensitivities to the MiniBooNE low-energy excess in the electron hypothesis for an exposure of the MicroBooNE detector of $13.2 \times 10^{20} \mathrm{POT}$. The uncertainties in the number of events include the systematic effects described in Section 7.

\begin{tabular}{lrrrr}
\hline Method & $\begin{array}{r}\text { Exp. signal } \\
\text { events }\end{array}$ & $\begin{array}{r}\text { Exp. bkg. } \\
\text { events }\end{array}$ & $\begin{array}{r}\text { Stat. only } \\
\text { significance }[\sigma]\end{array}$ & $\begin{array}{r}\text { Sys. and stat. } \\
\text { significance }[\sigma]\end{array}$ \\
\hline Rectangular & $17.3 \pm 4.2$ & $462.7 \pm 111.1$ & 1.25 & 0.83 \\
BDTs & $12.3 \pm 3.0$ & $298.9 \pm 71.7$ & 2.08 & 1.76 \\
\hline
\end{tabular}

While being informative, these values must be anyway interpreted very carefully for several reasons, listed below.

- The values of the rectangular cuts were not optimised on the low-energy excess signal, but on the $\nu_{e} \mathrm{CC} 0 \pi-\mathrm{Np}$ component. This result reflects our choice to be as agnostic as possible on the shape of the eventual low-energy excess signal.

- For the same reason, the BDTs were not trained on the low-energy excess signal. Their performances are then not optimised to select low-energy electron neutrinos.

- The limited amount of data in the unblinded sample (corresponding to around $3 \%$ of the expected amount of POT collected by MicroBooNE) does not allow us to validate stricter cuts.

- The systematic uncertainties are not optimised. In particular, the detector uncertainties are in our case dominated by the uncertainty on the space-charge effect, which will be greatly reduced once a full data-driven map is available.

- The flux and cross-section systematic uncertainties can be reduced by performing a combined $\nu_{e}+\nu_{\mu}$ analysis. In this case, the covariance matrix will contain also the selected $\nu_{\mu}$ events and the off-diagonal elements will correlate $\nu_{e}$ and $\nu_{\mu}$ events. In this way, it is possible also the reduce the effect of the 
cross-section uncertainty on the sensitivity, since $\nu_{\mu}$ and $\nu_{e}$ cross sections are closely related.

- Several improvements in the signal reconstruction, cosmic-ray rejection, and pattern recognition have been implemented in the software or will be implemented soon. An overview of these changes will be given in Section 8.3 .

\subsection{Future improvements}

\section{Cosmic Ray Tagger}

As seen in Section 6.2.8, the dominant source of events passing the pre-selection is represented by cosmic-ray interactions. The Cosmic Ray Tagger (CRT), described extensively in [93], offers several ways to reject these events at the pre-selection stage. First, a coincidence veto of in-time flashes in the PMTs and CRT would allow us to reject a significant background of data beam-off events. There is some danger that neutrino interactions are also vetoed by this coincidence, but that is unlikely for $\nu_{e}$ events, since most of the particles which exit the TPC and can hit the CRT are muons.

Additionally, for events where an out-of-TPC neutrino interaction creates a flash in time with the beam, but a cosmic interaction is matched to that flash, the CRT can also be useful. TPC-to-CRT matching of muon tracks can mitigate this background by flagging a TPC neutrino candidate object and allow us to reject out-of-time cosmic rays matched to an in-time, out-of-TPC neutrino flash.

Cosmic-ray rejection is also particularly important at low energy, where a Michel electron can often mimic the topology of an electron neutrino.

The CRT was not used in this analysis because it was not yet installed when the data sample analysed here was recorded. At as of January 2019, there are $5 \times 10^{20}$ POT collected without the CRT and more than $6 \times 10^{20}$ POT collected with the CRT. With the full approved running of MicroBooNE $\left(1.32 \times 10^{21}\right.$ POT $)$, we anticipate we will collect $8.2 \times 10^{20}$ POT with the CRT. 


\section{Event reconstruction improvements}

As shown in Section 6.2.7, the selection inefficiency depends on several factors. In particular, a better object reconstruction will allow to recover the events where reconstruction issues did not allow to satisfy the topology requirement (13.7\% of the events do not have showers reconstructed and 3.5\% have only one shower) or in which a cosmic ray is wrongly selected as neutrino candidate (7.9\%). Further improvements in reconstruction and selection can be made to reduce the cosmic contamination in the selected events (cosmic contaminated background). In particular, changes in the Pandora framework have been implemented which will improve the cosmic rejection and the neutrino selection efficiency. A preliminary study on the data beam-off sample shows an improvement in the cosmic rejection by a factor of 5 .

\section{Proton and electron particle identification}

The current performances of the selection rely essentially on the measured $d E / d x$ of the reconstructed showers to identify the electron in the event and on the measured $d E / d x$ of the reconstructed tracks to identify the protons. An improvement in the shower clustering and vertexing will directly cause an increase of the signal efficiency, since more electron showers will have a correctly measured $d E / d x$. At the moment, only the collection plane is used for calorimetric measurements. However, when the showers or the tracks are aligned to the collection plane, the number of reconstructed hits is not sufficient for the measurement of the $d E / d x$. A preliminary study shows that, using the induction planes, it is possible to have $30 \%$ more showers with a correctly measured $d E / d x$.

\subsection{Improvements and sensitivity}

It is possible to quantify the effect of selection efficiency and background-rejection improvements on the sensitivity to the low-energy excess. In Figure 8.5 we show the expected sensitivity (with and without systematic uncertainties) obtained by 


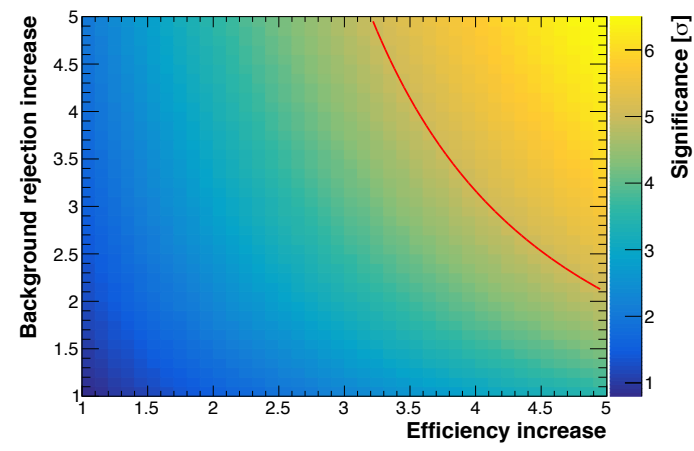

(a) Rectangular cuts, sys. uncertainties.

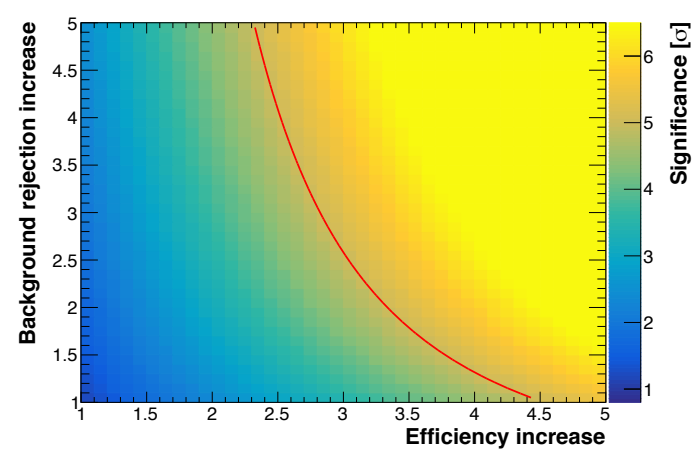

(c) Rectangular cuts, stat. uncertainties.

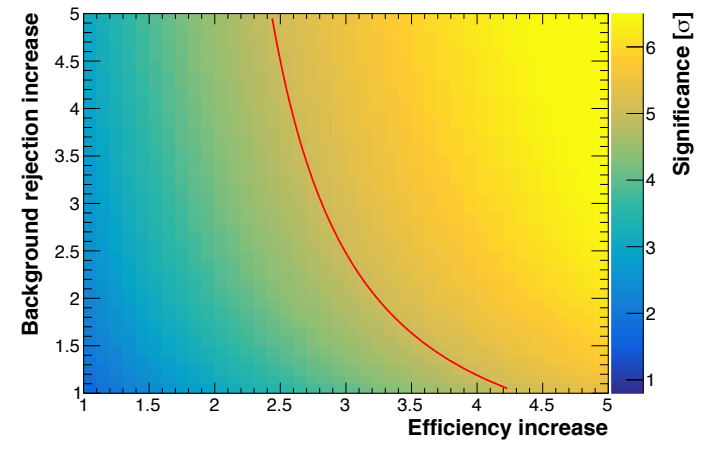

(b) BDTs cuts, sys. uncertainties.

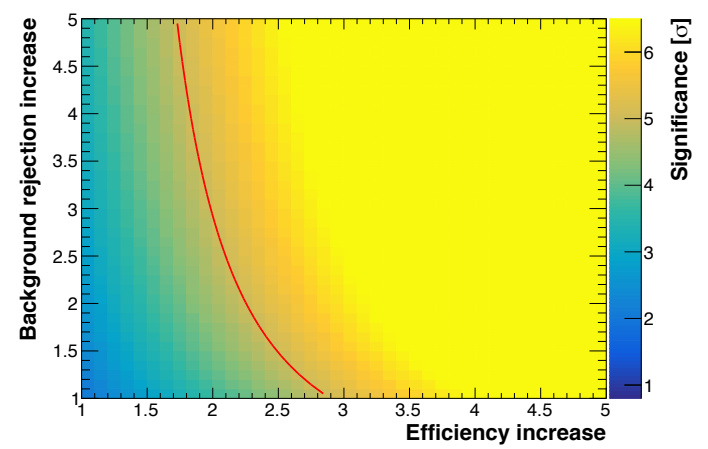

(d) BDTs cuts, stat. uncertainties.

Figure 8.5: Expected sensitivity to the low-energy excess in the electron hypothesis as a function of a constant scaling of our selection efficiency and background-rejection power, with (up) and without (bottom) systematic uncertainties. The red line corresponds to the $5 \sigma$ contour.

varying our efficiency and our background-rejection power by a constant amount, both for the rectangular and the BDTs cuts.

The distributions show that, with a reasonable improvement in our background rejection and selection efficiency, together with a better assessment of the systematic uncertainties, it is possible to achieve a sensitivity of $5 \sigma$ to the MiniBooNE lowenergy excess in the electron hypothesis.

A parallel analysis is currently being developed, which assumes that the excess is caused by an underestimation of the $\Delta \rightarrow N \gamma$ process. This process represents one of the main backgrounds of the MiniBooNE experiment, as described in Section 3.2 This analysis tries to select NC events with single photons in the final state 131. 


\section{9 \\ Conclusions}

The MicroBooNE experiment was designed to search and to clarify the nature of the low-energy excess of electron-like events observed by the MiniBooNE experiment.

We implemented an algorithm which employs the information coming from the optical system and the TPC of the MicroBooNE detector to select a sample enriched with $\nu_{e} \mathrm{CC} 0 \pi-\mathrm{Np}$ events. We showed that it is possible to reject the cosmic and neutrino backgrounds by applying rectangular cuts on kinematic and calorimetric variables or by exploiting the classification power of the Boosted Decision Trees. In particular, we showed that the energy loss per length $d E / d x$ can be used to distinguish between electrons and single photons interacting in a LArTPC.

The selection was applied on a small sub-sample of the data collected by triggering on the Booster Neutrino Beam, corresponding to $4.34 \times 10^{19}$ POT. In our Monte Carlo simulation we selected $3.2 \pm 0.8(4.4 \pm 1.2) \nu_{e} \mathrm{CC} 0 \pi$-Np events and $12.0 \pm 3.0(5.5 \pm 1.5)$ background events after the application of the rectangular cuts (BDTs cuts), in good agreement with the 16 (11) selected data events. The validation was performed on two orthogonal side-bands enriched with $\mathrm{NC}$ and $\mathrm{CC}$ $\nu_{\mu}$ interactions. The systematic uncertainties related to the cross-section, flux, and detector simulation were evaluated. The selection was also applied to an independent data sample acquired by triggering on the NuMI beam. The significance of the 
presence of electron neutrinos in the NuMI beam was calculated, giving solid confidence in the analysis presented.

We measured the current sensitivity to the low-energy excess with an expected exposure of the MicroBooNE detector of $13.2 \times 10^{20}$ POT. The performances needed to reach a $5 \sigma$ sensitivity were estimated.

The analysis showed here will serve as the foundation of the final low-energy excess search in the electron hypothesis. We have developed the full analysis framework, including the assessment of the systematic uncertainties, which can now be used with the improved simulations to measure the definitive MicroBooNE sensitivity to the low-energy excess.

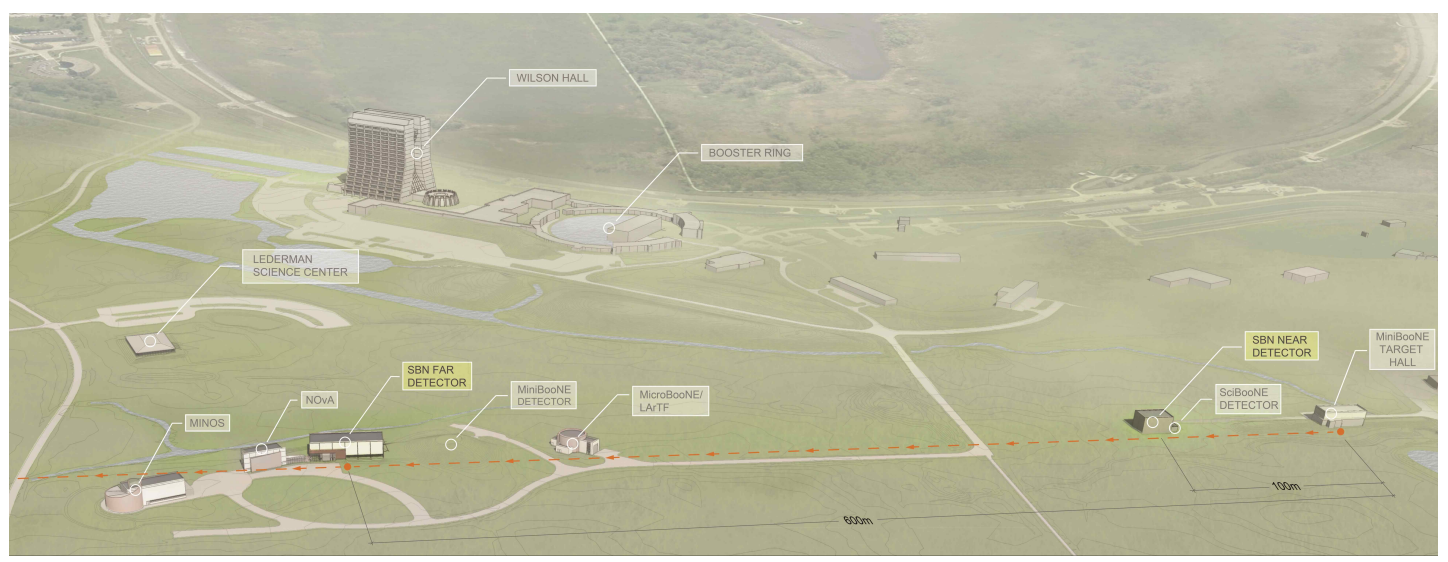

Figure 9.1: Aerial view of the Fermilab campus with the position of the three detectors forming the future Short Baseline Neutrino program. The neutrino beam target is placed on the right side of the picture. From $85 \mid$.

Regarding the future, MicroBooNE is the first stage of the broader Short Baseline Neutrino (SBN) program at Fermilab 85. In this program, two other LArTPCs will be placed on-axis with the Booster Neutrino Beamline. The Short Baseline Neutrino Detector (SBND, formerly known as LAr1-ND 132) will be placed close to the neutrino beam target and the refurbished ICARUS T600 detector [133] will be placed $600 \mathrm{~m}$ far from the target, $130 \mathrm{~m}$ farther than MicroBooNE (Figure 9.1).

The presence of three detectors on the same beamline employing the same technology will allow to constrain the flux, cross-section, and detector systematic uncertainties. The data coming from three detectors placed at different distances will allow to observe an eventual oscillation pattern and set the best limit on the search 


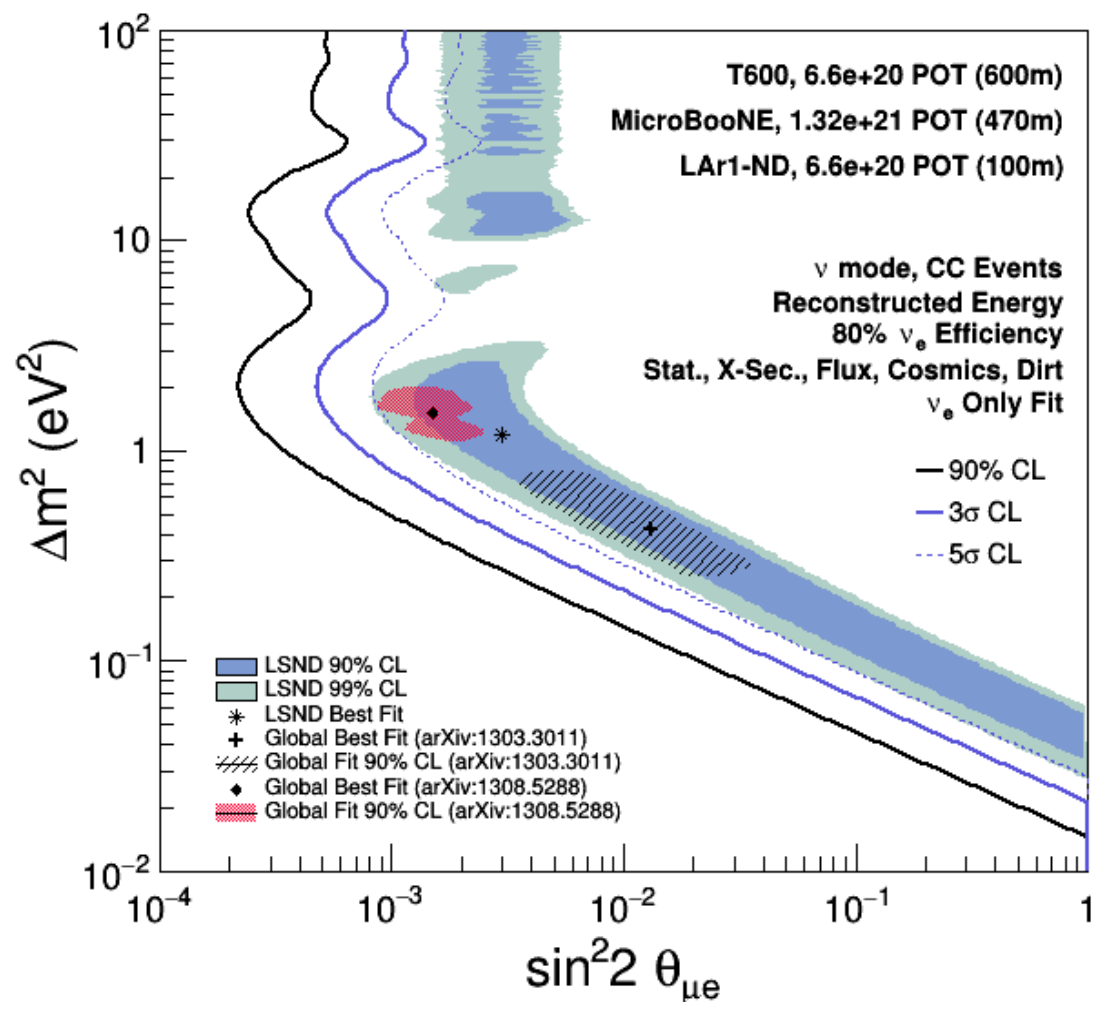

Figure 9.2: Sensitivity of the SBN Program to $\nu_{\mu} \rightarrow \nu_{e}$ oscillation signals. The filled areas correspond to the current allowed regions and the lines represent the sensitivity of the SBN program at different confidence levels. From 85 .

for sterile neutrino oscillations in the eV region. Figure 9.2 shows the sensitivity of the SBN program to $\nu_{\mu} \rightarrow \nu_{e}$ oscillations, assuming background rejection through topological cuts and with the aid of an external cosmic-ray tagger.

The next-generation large-scale neutrino experiment will be DUNE, which aims to precision measurement of the PMNS mixing angles and to identify the neutrino mass hierarchy. The ultimate goal is to verify the presence of $\mathrm{CP}$ violation in the leptonic sector 134. The design of DUNE employs 40 kton LArTPCs and the know-how with this technology acquired by MicroBooNE will be of fundamental importance for the success of the experiment. Addressing the presence of sterile neutrinos is also of the utmost importance for DUNE, as their existence would affect the interpretation of the oscillation patterns observed 135 . 
Appendices 


\section{Measurement of cosmic-ray reconstruction efficiencies in the MicroBooNE LArTPC using a small external cosmic-ray counter}




\section{Measurement of cosmic-ray reconstruction efficiencies in the MicroBooNE LArTPC using a small external cosmic-ray counter}

\section{The MicroBooNE collaboration}

R. Acciarri, ${ }^{g}$ C. Adams, ${ }^{c c, h}$ R. An, ${ }^{i}$ J. Anthony, ${ }^{c}$ J. Asaadi, ${ }^{z}$ M. Auger, ${ }^{a}$ L. Bagby, ${ }^{g}$ S. Balasubramanian, ${ }^{c c}$ B. Baller, ${ }^{g}$ C. Barnes, ${ }^{o}$ G. Barr, ${ }^{r}$ M. Bass, ${ }^{r}$ F. Bay, ${ }^{a}{ }^{a}$ M. Bishai, ${ }^{b}$ A. Blake, ${ }^{k}$ T. Bolton, ${ }^{j}$ L. Camilleri, ${ }^{f}$ D. Caratelli, ${ }^{f}$ B. Carls, ${ }^{g}$ R. Castillo Fernandez, ${ }^{g}$ F. Cavanna, ${ }^{g}$ H. Chen, ${ }^{b}$ E. Church, ${ }^{s}$ D. Cianci, ${ }^{m, f}$ E. Cohen, ${ }^{x}$ G.H. Collin, ${ }^{n}$ J.M. Conrad, ${ }^{n}$ M. Convery, ${ }^{v}$ J.I. Crespo-Anadón, ${ }^{f}$ M. Del Tutto, ${ }^{r}$ D. Devitt, ${ }^{k}$ S. Dytman, ${ }^{t}$ B. Eberly, ${ }^{v}$ A. Ereditato, ${ }^{a}$ L. Escudero Sanchez, ${ }^{c}$ J. Esquivel, ${ }^{w}$ A.A. Fadeeva, ${ }^{f}$ B.T. Fleming, ${ }^{c c}$ W. Foreman, ${ }^{d}$ A.P. Furmanski, ${ }^{m}$ D. Garcia-Gamez, ${ }^{m}$ G.T. Garvey, ${ }^{l}$ V. Genty, ${ }^{f}$ D. Goeldi, ${ }^{a}$ S. Gollapinni, ${ }^{j}, y$ N. Graf, ${ }^{t}$ E. Gramellini, ${ }^{c c}$ H. Greenlee, ${ }^{g}$ R. Grosso, ${ }^{e}$ R. Guenette, ${ }^{r, h}$ A. Hackenburg, ${ }^{c c}$ P. Hamilton, ${ }^{w}$ O. Hen, ${ }^{n}$ J. Hewes, ${ }^{m}$ C. Hill, ${ }^{m}$ J. Ho, ${ }^{d}$ G. Horton-Smith,${ }^{j}$ A. Hourlier, ${ }^{n}$ E.-C. Huang, ${ }^{l}$ C. James, ${ }^{g}$ J. Jan de Vries, ${ }^{c}$ C.-M. Jen, ${ }^{b b}$ L. Jiang, ${ }^{t}$ R.A. Johnson, ${ }^{e}$ J. Joshi, ${ }^{b}$ H. Jostlein, ${ }^{g}$ D. Kaleko, ${ }^{f}$ L.N. Kalousis, ${ }^{b b, 1}$ G. Karagiorgi, ${ }^{m, f}$ W. Ketchum,${ }^{g}$ B. Kirby, ${ }^{b}$ M. Kirby, ${ }^{g}$ T. Kobilarcik, ${ }^{g}$ I. Kreslo, ${ }^{a}$ G. Lange, ${ }^{b b}$ A. Laube, ${ }^{r}$ Y. Li, ${ }^{b}$ A. Lister, ${ }^{k}$ B.R. Littlejohn, ${ }^{i}$ S. Lockwitz, ${ }^{g}$ D. Lorca, ${ }^{a}$ W.C. Louis, ${ }^{l}$ M. Luethi, ${ }^{a}$ B. Lundberg, ${ }^{g}$ X. Luo, ${ }^{c c}$ A. Marchionni, ${ }^{g}$ C. Mariani, ${ }^{b b}$ J. Marshall, ${ }^{c}$ D.A. Martinez Caicedo, ${ }^{i}$ V. Meddage, ${ }^{j}$ T. Miceli, ${ }^{p}$ G.B. Mills, ${ }^{l}$ J. Moon, ${ }^{n}$ M. Mooney, ${ }^{b}$ C.D. Moore, ${ }^{g}$ J. Mousseau, ${ }^{o}$ R. Murrells, ${ }^{m}$ D. Naples, ${ }^{t}$ P. Nienaber, ${ }^{u}$ J. Nowak, ${ }^{k}$ O. Palamara, ${ }^{g}$ V. Paolone, ${ }^{t}$ V. Papavassiliou, ${ }^{p}$ S.F. Pate,${ }^{p}$ Z. Pavlovic, ${ }^{g}$ R. Pelkey, ${ }^{b}{ }^{b}$ E. Piasetzky, ${ }^{x}$ D. Porzio, ${ }^{m}$ G. Pulliam, ${ }^{w}$ X. Qian, ${ }^{b}$ J.L. Raaf, ${ }^{g}$ A. Rafique, ${ }^{j}$ L. Rochester, ${ }^{v}$ C. Rudolf von Rohr, ${ }^{a}$ B. Russell, ${ }^{c c}$ D.W. Schmitz, ${ }^{d}$ A. Schukraft, ${ }^{g}$ W. Seligman, ${ }^{f}$ M.H. Shaevitz, ${ }^{f}$ J. Sinclair, ${ }^{a}$ A. Smith, ${ }^{c}$ E.L. Snider, ${ }^{g}$ M. Soderberg, ${ }^{w}$ S. Söldner-Rembold, ${ }^{m}$ S.R. Soleti, ${ }^{r, 2}$ P. Spentzouris, ${ }^{g}$ J. Spitz, ${ }^{o}$ J.St. John, ${ }^{e}$ T. Strauss, ${ }^{g}$ A.M. Szelc, ${ }^{m}$ N. Tagg, ${ }^{q}$ K. Terao, ${ }^{f, v}$ M. Thomson, ${ }^{c}$ M. Toups, ${ }^{g}$ Y.-T. Tsai, ${ }^{v}$ S. Tufanli, ${ }^{c c}$ T. Usher, ${ }^{v}$ W. Van De Pontseele, ${ }^{r}$ R.G. Van de Water, ${ }^{l}$ B. Viren, ${ }^{b}$ M. Weber, ${ }^{a}$ D.A. Wickremasinghe, ${ }^{t}$ S. Wolbers, ${ }^{g}$ T. Wongjirad, ${ }^{n}$ K. Woodruff, ${ }^{p}$ T. Yang, ${ }^{g}$ L. Yates, ${ }^{n}$ G.P. Zeller, ${ }^{g}$ J. Zennamo ${ }^{d}$ and C. Zhang ${ }^{b}$

\footnotetext{
${ }^{a}$ Universität Bern, Bern CH-3012, Switzerland

${ }^{b}$ Brookhaven National Laboratory (BNL), Upton, NY, 11973, U.S.A.

${ }^{c}$ University of Cambridge, Cambridge CB3 OHE, United Kingdom
}

${ }^{1}$ Now at: Vrije Universiteit Brussel, 1050 Ixelles, Belgium.

${ }^{2}$ Corresponding author. 
${ }^{d}$ University of Chicago, Chicago, IL, 60637, U.S.A.

${ }^{e}$ University of Cincinnati, Cincinnati, OH, 45221, U.S.A.

${ }^{f}$ Columbia University, New York, NY, 10027, U.S.A.

${ }^{g}$ Fermi National Accelerator Laboratory (FNAL), Batavia, IL 60510, U.S.A.

${ }^{h}$ Harvard University, Cambridge, MA 02138, U.S.A.

${ }^{i}$ Illinois Institute of Technology (IIT), Chicago, IL 60616, U.S.A.

${ }^{j}$ Kansas State University (KSU), Manhattan, KS, 66506, U.S.A.

${ }^{k}$ Lancaster University, Lancaster LA1 4YW, United Kingdom

${ }^{l}$ Los Alamos National Laboratory (LANL), Los Alamos, NM, 87545, U.S.A.

${ }^{m}$ The University of Manchester, Manchester M13 9PL, United Kingdom

${ }^{n}$ Massachusetts Institute of Technology (MIT), Cambridge, MA, 02139, U.S.A.

${ }^{\circ}$ University of Michigan, Ann Arbor, MI, 48109, U.S.A.

${ }^{p}$ New Mexico State University (NMSU), Las Cruces, NM, 88003, U.S.A.

${ }^{q}$ Otterbein University, Westerville, OH, 43081, U.S.A.

${ }^{r}$ University of Oxford, Oxford OX1 3RH, United Kingdom

${ }^{s}$ Pacific Northwest National Laboratory (PNNL), Richland, WA, 99352, U.S.A.

${ }^{t}$ University of Pittsburgh, Pittsburgh, PA, 15260, U.S.A.

"Saint Mary's University of Minnesota, Winona, MN, 55987, U.S.A.

${ }^{v}$ SLAC National Accelerator Laboratory, Menlo Park, CA, 94025, U.S.A.

${ }^{w}$ Syracuse University, Syracuse, NY, 13244, U.S.A.

${ }^{x}$ Tel Aviv University, Tel Aviv, Israel, 69978

${ }^{y}$ University of Tennessee, Knoxville, TN, 37996, U.S.A.

${ }^{z}$ University of Texas, Arlington, TX, 76019, U.S.A.

${ }^{a}$ TUBITAK Space Technologies Research Institute, METU Campus, TR-06800, Ankara, Turkey

${ }^{b b}$ Center for Neutrino Physics, Virginia Tech, Blacksburg, VA, 24061, U.S.A.

${ }^{c c}$ Yale University, New Haven, CT, 06520, U.S.A.

E-mail: stefano.soleti@physics.ox.ac.uk

Abstract: The MicroBooNE detector is a liquid argon time projection chamber at Fermilab designed to study short-baseline neutrino oscillations and neutrino-argon interaction cross-section. Due to its location near the surface, a good understanding of cosmic muons as a source of backgrounds is of fundamental importance for the experiment. We present a method of using an external $0.5 \mathrm{~m}(\mathrm{~L}) \times 0.5 \mathrm{~m}(\mathrm{~W})$ muon counter stack, installed above the main detector, to determine the cosmic-ray reconstruction efficiency in MicroBooNE. Data are acquired with this external muon counter stack placed in three different positions, corresponding to cosmic rays intersecting different parts of the detector. The data reconstruction efficiency of tracks in the detector is found to be $\epsilon_{\text {data }}=(97.1 \pm 0.1$ (stat) \pm 1.4 (sys) $) \%$, in good agreement with the Monte Carlo reconstruction efficiency $\epsilon_{\mathrm{MC}}=(97.4 \pm 0.1) \%$. This analysis represents a small-scale demonstration of the method that can be used with future data coming from a recently installed cosmic-ray tagger system, which will be able to tag $\approx 80 \%$ of the cosmic rays passing through the MicroBooNE detector.

Keywords: Performance of High Energy Physics Detectors; Time projection chambers; Data reduction methods; Neutrino detectors

ArXiv ePrint: 1707.09903 


\section{Contents}

1 Introduction 1

2 The Muon Counter Stack 3

3 Data reduction and Monte Carlo simulation 3

3.1 Data sample reduction 3

3.2 Monte Carlo sample generation $\quad 6$

4 Reconstruction efficiencies $\quad 7$

4.1 Reconstruction efficiency measurement procedure $\quad 7$

4.2 Systematic uncertainties 10

4.2.1 Effect of the space-charge effect correction 11

4.2.2 Effect of the $d_{\max }$ requirement 11

4.2.3 Decay-in-flight or captured muons 11

4.2.4 Detector non-uniformities 11

$\begin{array}{lll}\text { 4.2.5 Energy sampling } & 12\end{array}$

4.3 Data/Monte Carlo comparison $\quad 12$

5 Conclusions $\quad 14$

\section{Introduction}

MicroBooNE (Micro Booster Neutrino Experiment) is a liquid argon time projection chamber (LArTPC) located at the Fermi National Accelerator Laboratory (Fermilab) [1]. The main physics goals of the experiment are to investigate the excess of low-energy events observed by the MiniBooNE collaboration [2] and to measure neutrino-argon interaction cross sections. MicroBooNE also provides important research and development contributions to detector technology and event reconstruction techniques for future LArTPC experiments, such as DUNE (Deep Underground Neutrino Experiment) [3]. The MicroBooNE detector is located $470 \mathrm{~m}$ downstream of the Booster Neutrino Beam (BNB) target. The BNB is predominantly composed of muon neutrinos $\left(v_{\mu}\right)$ with a peak neutrino energy at about $0.7 \mathrm{GeV}$.

The MicroBooNE detector consists of a rectangular time projection chamber (TPC) with dimensions of $256 \mathrm{~cm}$ (width) $\times 233 \mathrm{~cm}$ (height) $\times 1037 \mathrm{~cm}$ (length). The cylindrical cryostat contains a total of $170 \mathrm{t}$ of liquid argon, while the mass of liquid argon in the active volume, defined as the portion of the argon encompassed by the TPC, is $89 \mathrm{t}$. Figure 1 shows a graphical representation of the TPC in the MicroBooNE coordinate system. The $x$ direction corresponds to the drift coordinate, the $y$ direction is the vertical direction, and the $z$ direction points along the beam. 


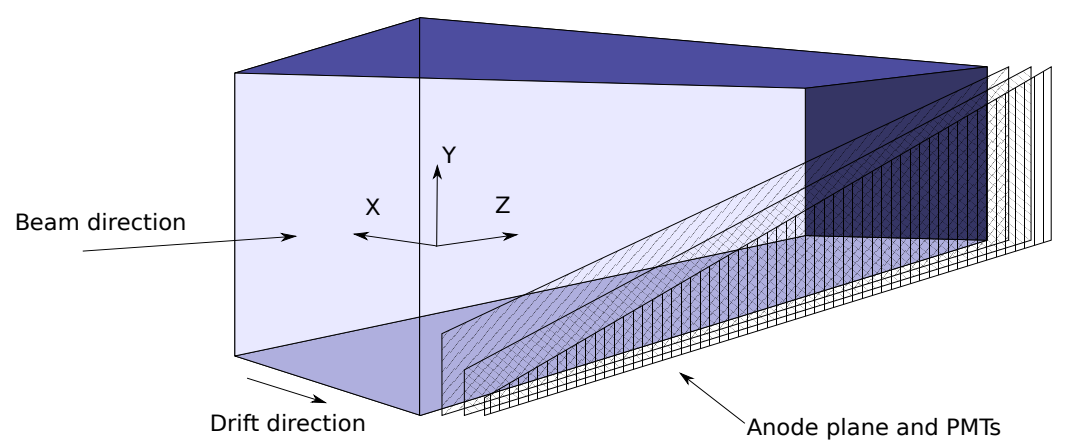

Figure 1. The MicroBooNE coordinate system. The three wire planes, shown in the right front face, are vertical (collection plane) and at $\pm 60^{\circ}$ to the vertical (induction planes). The dimensions of the TPC are $256 \mathrm{~cm} \times 233 \mathrm{~cm} \times 1037 \mathrm{~cm}$ in the $x, y$, and $z$ directions, respectively.

The TPC consists of three wire planes with $3 \mathrm{~mm}$ spacing at angles of $0^{\circ},+60^{\circ}$ and $-60^{\circ}$ with respect to the vertical. The cathode, made of a plane of stainless steel panels, operates at a voltage of $-70 \mathrm{kV}$. In a neutrino interaction, a neutrino from the beam interacts with an argon nucleus, and the secondary charged particles traverse the medium, losing energy and leaving an ionization trail. The resulting ionization electrons drift to the wire planes under an electric field of $273 \mathrm{~V} / \mathrm{cm}$. The distance between the cathode and anode is $2.56 \mathrm{~m}$. An ionization electron takes about $2.3 \mathrm{~ms}$ to travel the full drift distance, called the drift time window. Charge drifting past a wire plane induces a current that produces a bipolar signal in the electronics. The first two planes are referred to as induction planes. The wire plane furthest from the cathode has wires oriented vertically. Drifting electrons are collected on this plane producing a unipolar signal. Charge deposited in the TPC generates a signal used to create three distinct two-dimensional views (in terms of wire and time) of the event, which can be combined to reconstruct a three-dimensional image of the interaction. A set of 32 photomultipliers tubes (PMTs) is placed behind the anode plane to collect the argon scintillation light. Scintillation light provides timing information with few-ns precision, which provides the TPC start time of the event and can be used for background suppression. More details about the MicroBooNE detector can be found in ref. [1].

The detector is placed in a pit $6 \mathrm{~m}$ below the surface with no overburden. The muon cosmic-ray rate in the MicroBooNE detector is estimated to be $5.5 \mathrm{kHz}$, which corresponds to $\approx 13$ muons per TPC drift time window of $2.3 \mathrm{~ms}$. The abundant flux of cosmic muons is a source of background to neutrino events, and an optimal reconstruction of the cosmic rays in the TPC is therefore crucial.

In order to study the challenges of cosmic-ray background rejection in a surface neutrino experiment, the MicroBooNE detector was equipped with an external muon counter stack (MuCS) at the start of operations in 2015. We use this system to develop and demonstrate muon tagging. It also provides an external set of data to validate simulation and reconstruction. In the future, the method described in this paper will be applied to the data coming from the cosmic ray tagger (CRT) system [4], installed in March 2017. It is able to tag approximately $80 \%$ of the cosmic rays traversing the MicroBooNE LArTPC, which is an order of magnitude more than the coverage provided by the MuCS. This increased coverage of the incoming cosmic-ray flux the CRT provides allows to determine efficiencies over the full detector volume, to measure e.g. the cosmic-ray flux in the LArTPC. 


\section{The Muon Counter Stack}

The Muon Counter Stack, described in detail in ref. [5], consists of two sets of planar modules placed into two separate, light-tight boxes. The upper and lower boxes are placed $2.75 \mathrm{~m}$ and $2.03 \mathrm{~m}$ above the TPC, respectively. Their position is known to a precision of $0.5 \mathrm{~cm}$. Each planar module is constructed using 48 scintillator strips of $4 \mathrm{~cm}$ width, $48 \mathrm{~cm}$ length, and $2 \mathrm{~cm}$ thickness. The scintillator strips are arranged into a pair of bi-layers, each 12 strips wide and oriented perpendicular to each other. The overall setup is shown in figure 2 .

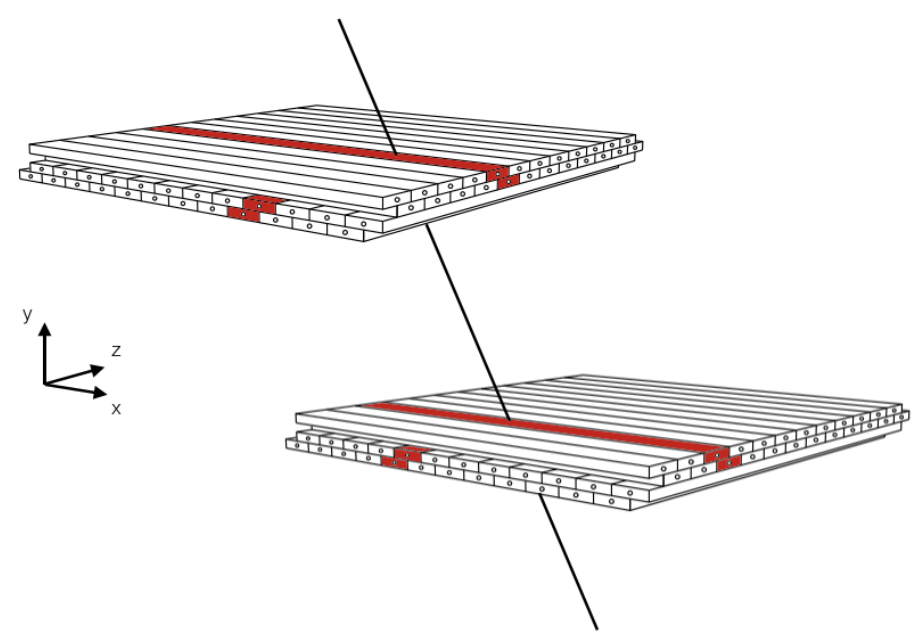

Figure 2. A cosmic ray passing through the MuCS boxes hits the scintillator strips. We have a MuCS hit when the signal corresponding to the strip is above a certain threshold. The position of the panels along the $y$ axis and the position of the hit strips (highlighted in red) are used to extrapolate the trajectory of the cosmic ray down to the TPC located below the counters.

Each strip contains a wavelength shifting optical fiber, connected to a multi-anode PMT, which is read out by a dedicated DAQ system that records the hit patterns of the scintillator strips. The MuCS is designed to provide a trigger on through-going muons that intersect all four bi-layers of scintillator strips.

The data used in our analysis has been acquired with the MuCS in three different geometrical configurations. The three configurations correspond to a setup with the two boxes placed above the TPC and: (1) at the upstream end, (2) at the center, and (3) at the downstream end of the MicroBooNE detector, keeping the box spacing and alignment identical. A three-dimensional drawing of the three MuCS configurations is shown in figure 3.

\section{Data reduction and Monte Carlo simulation}

\subsection{Data sample reduction}

The MuCS trigger is propagated to the MicroBooNE trigger board and provides the starting time $\left(t_{0}\right)$ of a track in the TPC associated with the MuCS. The MuCS triggers at a rate of nearly $3 \mathrm{~Hz}$. 


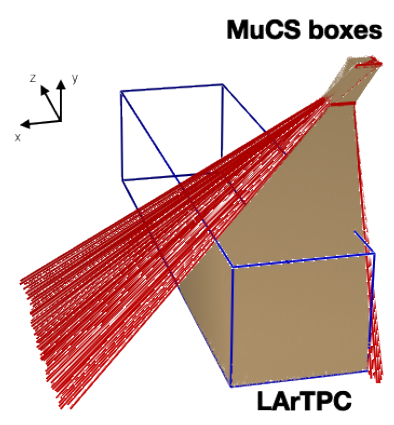

(a) Upstream

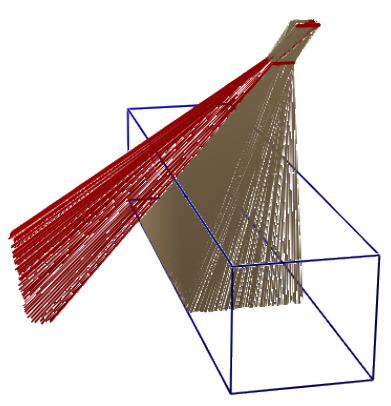

(b) Center

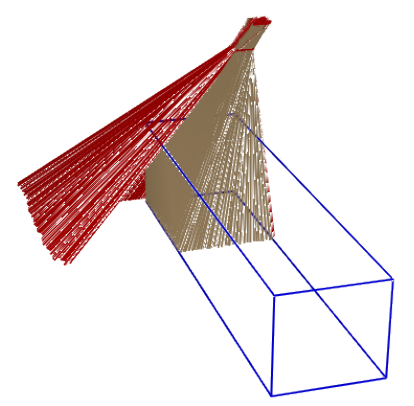

(c) Downstream

Figure 3. Illustration of the three MuCS configurations with a Monte Carlo simulation of the possible MuCS cosmic-ray trajectories. Brown tracks correspond to cosmic rays hitting both the MuCS boxes and TPC, while red tracks traverse only the MuCS and miss the TPC.

A software filter removes events with more than 4 hit strips per bi-layer from the data sample, discarding events with unclear hit patterns caused by electronic noise or air showers. After this filter, the MuCS data sample consists of $\approx 30000$ MuCS-triggered events, around 10000 for MuCS configuration, acquired in a total of $\sim 10$ hours of data taking. The probability that a second cosmic ray crosses the MuCS and hits the TPC during the same drift time window of $2.2 \mathrm{~ms}$ is negligible for this study, given our trigger rate of $3 \mathrm{~Hz}$. Therefore, we assume for this study that only one cosmic ray per drift time window is traversing the MuCS and the TPC. The data follow a processing path that merges the MuCS hit patterns and extrapolated trajectory information with the TPC to form a MuCS-merged dataset.

As illustrated in figure 2, when a signal in one strip is above a certain threshold we have a MuCS hit. By combining the MuCS hits in each bi-layer, we obtain two sets of position coordinates of the crossing points of the cosmic rays (the $z$ and $x$ coordinates in the MicroBooNE TPC reference frame shown in figure 1). The height at which the modules are positioned (corresponding to the $y$ coordinate for the MicroBooNE TPC reference frame) allows the extrapolation of a threedimensional trajectory of the cosmic ray from the MuCS down to the TPC, which is defined as a MuCS-extrapolated track.

The starting angle of the cosmic ray trajectory, in spherical coordinates, is defined by:

$$
\theta=\operatorname{acos}\left(\frac{z_{\text {bottom }}-z_{\text {top }}}{r}\right), \quad \phi=\operatorname{atan}\left(\frac{y_{\text {bottom }}-y_{\text {top }}}{x_{\text {bottom }}-x_{\text {top }}}\right),
$$

where $r$ is the distance between $\left(x_{\mathrm{top}}, y_{\mathrm{top}}, z_{\mathrm{top}}\right)$ and $\left(x_{\mathrm{bottom}}, y_{\mathrm{bottom}}, z_{\mathrm{bottom}}\right)$, given by the hit positions in the top and bottom MuCS box, respectively.

In the TPC, ionization electrons from cosmic ray muons passing through the MicroBooNE cryostat are drifted to the wires and TPC hits are extracted, which are then used by the track reconstruction algorithms provided by the Pandora framework [6] to form TPC reconstructed tracks.

The Pandora reconstruction produces as a first stage a list of two-dimensional clusters, that represent continuous, unambiguous lines of hits. Thus, cluster-merging algorithms identify associ- 


\section{A. Measurement of cosmic-ray reconstruction efficiencies in the MicroBooNE}

ations between multiple clusters. The three-dimensional track reconstruction then collects the twodimensional clusters from the three readout planes that represent individual, track-like particles [12].

The build-up of slow-moving positive ions in a detector due to ionization from cosmic rays leads to a small distortion of the electric field in the detector (space-charge effect [7]). This effect causes a displacement in the reconstructed position of signal ionization electrons in LArTPCs. We correct the reconstructed track end points in the TPC vertically to lie on the boundary of the TPC.

At the top and bottom boundary of the TPC, this distortion leads primarily to a vertical displacement of the ionization tracks. The vertical displacements are larger far from the anode, around $10 \mathrm{~cm}$, (due to the longer travel distance of the ionization electrons) and for positions far from the center of the TPC (where the built-up charge increases the distortion). The vertical displacement at the top and bottom boundary of the TPC has been measured in the data by reconstructing the start and end points of minimum-ionizing particles crossing the TPC from a sample of MuCS-triggering cosmic-rays, while the vertical displacement in the TPC bulk has been estimated by a dedicated simulation, described in ref. [11]. Knowing in this way the magnitude of the effect, it has been possible to correct it in the data, allowing direct comparison with the current Monte Carlo simulation.

The first intersection point between the MuCS-extrapolated track and the TPC is defined as ( $\left.x_{\mathrm{MuCS}}, y_{\mathrm{MuCS}}, z_{\mathrm{MuCS}}\right)$. However, because of multiple Coulomb scattering in the material between the MuCS boxes and the TPC, the starting point of the reconstructed track in the TPC corresponding to the MuCS-triggering cosmic ray does not coincide exactly with the extrapolated intersection point.

The TPC reconstructed track with the closest starting point to the MuCS-extrapolated track intersection point is selected for further analysis and is defined as a MuCS-tagged track. The distance $d$ between the extrapolated intersection point and the starting point of the MuCS-tagged track is defined as

$$
d=\sqrt{\left(x_{\mathrm{MuCS}}-x_{\mathrm{reco}}\right)^{2}+\left(y_{\mathrm{MuCS}}-y_{\mathrm{reco}}\right)^{2}+\left(z_{\mathrm{MuCS}}-z_{\mathrm{reco}}\right)^{2}},
$$

where $\left(x_{\text {reco }}, y_{\text {reco }}, z_{\text {reco }}\right)$ is the starting point of the MuCS-tagged track.

The data will then include two different sets of information:

- MuCS-extrapolated information: a line crossing the entire TPC is extrapolated from the two points given by the MuCS (one for each box). From this extrapolated line it is possible to obtain: (1) the two extrapolated starting angles $\theta$ and $\phi$ of the MuCS, defined in eq. (3.1), (2) the extrapolated start point ( $\left.x_{\mathrm{MuCS}}, y_{\mathrm{MuCS}}, z_{\mathrm{MuCS}}\right)$ described above, and (3) the extrapolated end point, corresponding to point where the MuCS-extrapolated track exits the TPC. The extrapolated track length $L$ is calculated by measuring the distance between the extrapolated start point and the extrapolated end point.

- Reconstructed TPC data information: for each MuCS-triggered event, the reconstructed starting point $\left(x_{\text {reco }}, y_{\text {reco }}, z_{\text {reco }}\right)$ of the MuCS-tagged track.

To remove events where the cosmic-ray muon triggered the MuCS but did not hit the TPC, or crossed it for a very short path, we require an extrapolated length in the TPC of $L>20 \mathrm{~cm}$. 

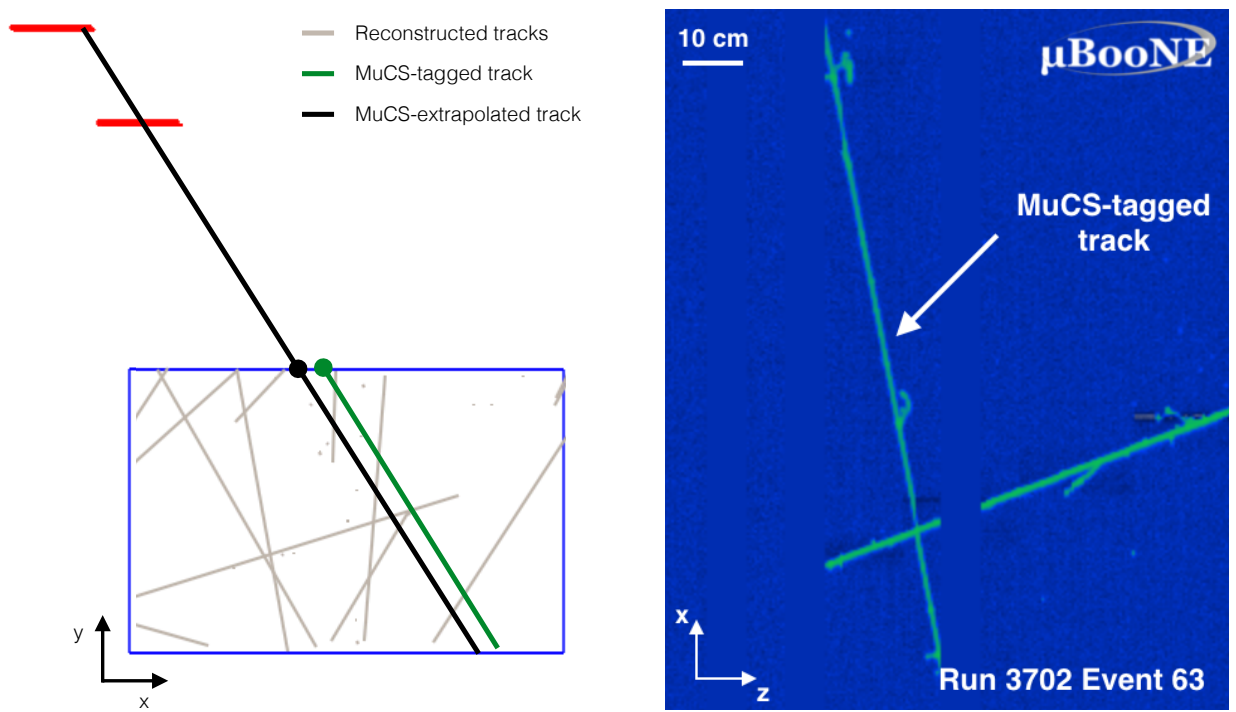

Figure 4. Left: two-dimensional schematic view of a MuCS event in MicroBooNE. The black line shows the MuCS-extrapolated track, while the green line corresponds to the MuCS-tagged track. The black and green dots correspond to the ( $\left.x_{\mathrm{MuCS}}, y_{\mathrm{MuCS}}, z_{\mathrm{MuCS}}\right)$ and $\left(x_{\mathrm{reco}}, y_{\text {reco }}, z_{\text {reco }}\right)$ coordinates, respectively. Right: example of a MicroBooNE event display for the collection plane, showing a MuCS-tagged track in a data event.

\subsection{Monte Carlo sample generation}

The Monte Carlo sample consists of a simulation of cosmic ray events in the MicroBooNE TPC. The cosmic rays are generated using the CORSIKA 7.4003 [8] simulation software. The muons are propagated through the detector using GEANT 4.9.6 [9] and passed through a detector simulation stage, developed using the LArSoft framework [10]. The detector simulation reproduces the electron drift, the induction and collection of signals on wires, and the electronics response. The simulation also includes information on the state of the detector readout. Noisy or unresponsive wires, for example, can complicate track reconstruction. The impact of their effect is discussed in section 4.2.4.

The direction of the simulated cosmic ray is given by its momentum when it enters the TPC, given by GEANT4. The starting angles $\theta$ and $\phi$ are defined in this case as

$$
\theta=\operatorname{acos}\left(\frac{p_{z}}{p}\right), \quad \phi=\operatorname{atan}\left(\frac{p_{y}}{p_{x}}\right),
$$

where $p_{x}, p_{y}, p_{z}$ are the $x, y, z$ components of the cosmic-ray momentum of magnitude $p$. The track length $L$ is calculated by extrapolating a straight line through the TPC in the $\theta, \phi$ direction and measuring the distance from the true entering point to the extrapolated exiting point in the TPC.

This Monte Carlo simulation provides cosmic rays entering the TPC from all possible directions, while the cosmic rays triggering the MuCS can have only $\theta, \phi$ starting angles within the geometrical constraints of the system. Thus, cosmic rays in the Monte Carlo dataset are selected to match the $(\theta, \phi)$ parameter space covered by the MuCS-extrapolated tracks in data and the Monte Carlo events have been weighted to match the data distributions. 


\section{Reconstruction efficiencies}

\subsection{Reconstruction efficiency measurement procedure}

The reconstruction efficiency $\epsilon$ is defined as the fraction of MuCS-triggered cosmic-ray events that have a reconstructed track in the TPC:

$$
\epsilon=\frac{\text { reco. MuCS cosmic-ray events }}{\text { MuCS triggered events }}=\frac{M_{e}}{T_{e}} .
$$

In data, the MuCS-tagged track is defined as the reconstructed track with the closest starting point $\left(x_{\text {reco }}, y_{\text {reco }}, z_{\text {reco }}\right)$ to the extrapolated MuCS starting point $\left(x_{\mathrm{MuCS}}, y_{\mathrm{MuCS}}, z_{\mathrm{MuCS}}\right)$, as shown in figure 4.

The efficiency here does not quantify the accuracy of the track reconstruction in the TPC, such as the correctness of the track length or angle.

In order to limit the accidental misassociation of MuCS-triggered cosmic-ray muons with other nearby reconstructed tracks in the TPC, a selection requirement is placed on the maximum distance $d_{\text {max }}$ between the two points, $\left(x_{\text {reco }}, y_{\text {reco }}, z_{\text {reco }}\right)$ and ( $\left.x_{\text {MuCS }}, y_{\text {MuCS }}, z_{\text {MuCS }}\right)$. To study the dependence of the number of MuCS-tagged tracks on $d_{\max }$, a dedicated Monte Carlo simulation of a MuCS run is performed, defined as MuCS Monte Carlo, which is different from the Monte Carlo sample described in section 3.2. Each event of this simulation has one cosmic-ray muon passing through the MuCS boxes overlaid on a full simulation of cosmic rays in the TPC.

We use the truth information in the MuCS Monte Carlo simulation to determine if the identified MuCS-tagged cosmic ray corresponds to the true track from the cosmic muon or if it is an incorrectly associated cosmic ray, to which the extrapolated starting point distance is closer than $d_{\max }$ because of multiple Coulomb scattering. In the MuCS Monte Carlo the distance $d$ is defined as

$$
d=\sqrt{\left(x_{\text {sim }}-x_{\text {reco }}\right)^{2}+\left(y_{\text {sim }}-y_{\text {reco }}\right)^{2}+\left(z_{\text {sim }}-z_{\text {reco }}\right)^{2}}
$$

where $\left(x_{\text {sim }}, y_{\text {sim }}, z_{\text {sim }}\right)$ and $\left(x_{\text {reco }}, y_{\text {reco }}, z_{\text {reco }}\right)$ are the coordinates of the intersection of the simulated cosmic-ray trajectory with the TPC and of the closest reconstructed track, respectively. Figure 5 shows the distribution of the distance $d$ between the extrapolated starting point and the closest reconstructed starting point, for both data and MuCS Monte Carlo. In the reconstruction efficiency definition for the MuCS Monte Carlo sample, we replace the number of MuCS-triggered events in eq. (4.1) with the number of simulated MuCS events.

The efficiency $\epsilon_{\mathrm{tag}}$ of the $d_{\max }$ requirement is defined as

$$
\epsilon_{\mathrm{tag}}=\frac{\text { events with a reco. cosmic ray within } d_{\max }}{\text { MuCS triggered events }}=\frac{R_{e}\left(d_{\max }\right)}{T_{e}} .
$$

It is calculated for the data sample $\left(\epsilon_{\mathrm{tag}}^{\mathrm{data}}\right)$ and for the MuCS Monte Carlo sample $\left(\epsilon_{\mathrm{tag}}^{\mathrm{MuCS}-\mathrm{MC}}\right)$ by replacing the number of MuCS-triggered events with the number of simulated MuCS events.

The purity $P$ of the Monte Carlo MuCS sample, which represents the fraction of correctly tagged MuCS cosmic rays, is defined as the ratio between the number of events with a reconstructed MuCS cosmic ray correctly identified within $d_{\max }$ and the number of events with a reconstructed cosmic ray within $d_{\max }$ (MuCS-tagged cosmic rays):

$$
P=\frac{\text { events with a reco. MuCS cosmic ray within } d_{\max }}{\text { events with a reco. cosmic ray within } d_{\max }}=\frac{M_{e}\left(d_{\max }\right)}{R_{e}\left(d_{\max }\right)} .
$$




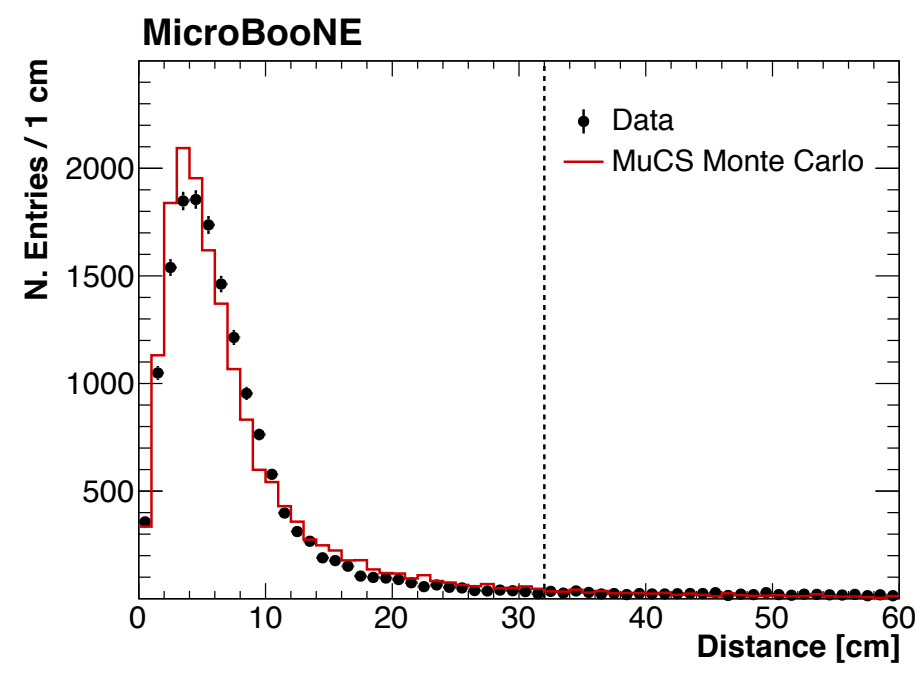

Figure 5. Data and MuCS Monte Carlo distributions of the distance $d$ between the extrapolated starting point and the closest reconstructed starting point for cosmic ray tracks passing through both the MuCS and the MicroBooNE TPC. The dashed line correspond to the $d_{\max }$ requirement $(32 \mathrm{~cm})$ chosen for this analysis.

The acceptance $A$ of the $d_{\max }$ requirement, which represents the portion of MuCS cosmic rays within $d_{\max }$, is defined as the ratio between the number of events with a reconstructed MuCS cosmic ray within $d_{\max }$ range and the total number of events with a reconstructed MuCS cosmic ray:

$$
A=\frac{\text { events with a reco. MuCS cosmic ray within } d_{\max }}{\text { events with a reco. MuCS cosmic ray }}=\frac{M_{e}\left(d_{\max }\right)}{M_{e}} .
$$

The acceptance of the $d_{\max }$ requirement is mainly affected by the multiple Coulomb scattering in the material between the MuCS and the TPC.

The reconstruction efficiency, as defined in eq. (4.1), is obtained, both for data $\left(\epsilon_{\text {data }}\right)$ and for MuCS Monte Carlo ( $\left.\epsilon_{\mathrm{MuCS}-\mathrm{MC}}\right)$, by

$$
\epsilon=\frac{M_{e}}{T_{e}}=\frac{R_{e}\left(d_{\max }\right)}{T_{e}} \times \frac{M_{e}\left(d_{\max }\right)}{R_{e}\left(d_{\max }\right)} \times \frac{M_{e}}{M_{e}\left(d_{\max }\right)}=\epsilon_{\mathrm{tag}} \times \frac{P}{A},
$$

where the $P / A$ ratio is taken only from the MuCS Monte Carlo simulation, while $\epsilon_{\mathrm{tag}}$ is measured with the data, $\epsilon_{\text {tag }}^{\mathrm{data}}$, or with the MuCS Monte Carlo simulation, $\epsilon_{\mathrm{tag}}^{\mathrm{MuCS}-\mathrm{MC}}$.

Figure 6 shows the tagging efficiency both for data $\left(\epsilon_{\mathrm{tag}}^{\mathrm{data}}\right)$ and MuCS Monte Carlo $\left(\epsilon_{\mathrm{tag}}^{\mathrm{MuCS}-\mathrm{MC}}\right)$, the purity $P$ and the acceptance $A$ as a function of $d_{\max }$. The reconstruction efficiencies for data $\left(\epsilon_{\text {data }}\right)$ and MuCS Monte Carlo $\left(\epsilon_{\text {MuCS-MC }}\right)$ are also shown.

Using eq. (4.1), the MuCS Monte Carlo reconstruction efficiency $\epsilon_{\text {MuCS-MC }}$ will not depend, by construction, on the chosen value of $d_{\max }$. Since the $P / A$ correction factor is determined by a Monte Carlo simulation, the data reconstruction efficiency $\epsilon_{\text {data }}$ has a small dependence on $d_{\max }$ (see figure 6), because of the small difference between $\epsilon_{\mathrm{tag}}^{\mathrm{data}}$ and $\epsilon_{\mathrm{tag}}^{\mathrm{MuCS}-\mathrm{MC}}$. The difference between the lowest and the highest value of $\epsilon_{\text {data }}$ is $0.2 \%$. This value is used to estimate the systematic uncertainty related to the $P / A$ correction factor, as further discussed in section 4.2. Figure 5 and 


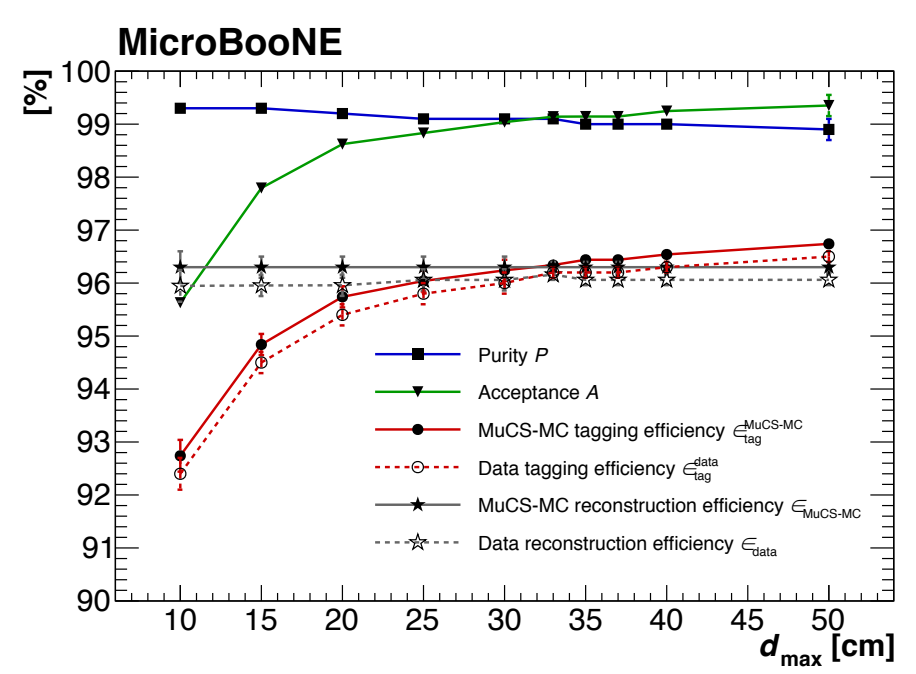

Figure 6. Data $\left(\epsilon_{\mathrm{tag}}^{\mathrm{data}}\right)$ and Monte Carlo $\left(\epsilon_{\mathrm{tag}}^{\mathrm{MC}}\right)$ tagging efficiency (red), purity $P$ (blue) and acceptance $A$ (green), as a function of $d_{\max }$. The MuCS reconstruction efficiencies for data $\left(\epsilon_{\text {data }}\right)$ and MuCS Monte Carlo $\left(\epsilon_{\mathrm{MuCS}-\mathrm{MC}}\right)$ are also shown as a reference (grey).

figure 6 show that the $d, P$ and $A$ distributions are constant around $d_{\max }=32 \mathrm{~cm}$, and the ratio $P / A$ is $\approx 1$. Therefore, we choose $32 \mathrm{~cm}$ as the value of $d_{\max }$. However, different values could be used.

To verify if the data reconstruction efficiency, measured in specific locations of the detector, is valid throughout the detector, we perform a direct comparison of the MuCS data with the Monte Carlo sample, using the Monte Carlo distribution generated as described in section 3.2. The Monte Carlo cosmic-ray reconstruction efficiency is defined as

$$
\epsilon_{\mathrm{MC}}=\frac{\text { reco. cosmic-ray tracks }}{\text { generated cosmic rays }} \text {. }
$$

This Monte Carlo sample contains cosmic rays generated over the entire TPC volume and therefore averages over any dependence of $\epsilon_{\mathrm{MC}}$ on the position of the cosmic ray in the TPC. The MuCS dataset, however, covers only three regions of the detector shown in figure 3.

A cosmic ray with a longer path in the TPC will correspond in general to a larger number of hit wires and thus to a higher reconstruction efficiency [12]. The reconstruction efficiency depends also on the direction of the cosmic ray, since cosmic rays parallel to the wires of one plane $\left(0^{\circ}, \pm 60^{\circ}\right.$ with respect to the $y$ axis $)$ will generate fewer hits in that particular plane, making the track reconstruction algorithm less efficient. We therefore express the data and the Monte Carlo reconstruction efficiencies, $\epsilon_{\mathrm{data}}$ and $\epsilon_{\mathrm{MC}}$, as a function of the starting spherical angles $\theta, \phi$ and of the expected track length $L$ in the TPC, as described in section 3.

The efficiency is plotted as a three-dimensional histogram in figure 7 , where each bin corresponds to a particular combination of the $\theta, \phi$, and $L$ variables. Bin width is chosen large enough to have a statistical uncertainty of less than $10 \%$ for every $(\theta, \phi, L)$ bin. The same $P / A$ correction factor is applied to every bin.

The data reconstruction efficiency $\epsilon_{\text {data }}$ does not take into account muons triggering the MuCS that decay or are captured before reaching the TPC. These events are counted in the denominator 
of eq. (4.1) and therefore lower the reconstruction efficiency since they have not reached the TPC and cannot be reconstructed. The muons travel on average $\approx 3 \mathrm{~m}$ between the top MuCS panel and the TPC: they cross approximately $\approx 10 \mathrm{~cm}$ of scintillator materials, $\approx 2.5 \mathrm{~m}$ of air, $\approx 1 \mathrm{~cm}$ of steel and $\approx 0.5 \mathrm{~m}$ of liquid argon. The fraction $D$ of cosmic rays that traverse the MuCS but decay or are captured before reaching the TPC is calculated from the MuCS Monte Carlo simulation as

$$
D=\frac{\text { decayed } / \text { captured muons }}{\text { MuCS triggered events }}=(1.0 \pm 0.1) \% \text {. }
$$

This correction factor does not show a dependence of $\theta, \phi$, and $L$ with the present level of statistics. The corrected data reconstruction efficiency is given by

$$
\epsilon_{\text {data }}^{\text {corr }}=\frac{\epsilon_{\text {data }}}{1-D} .
$$

Figure 7 shows both the corrected data and the Monte Carlo reconstruction efficiency in the $3 \mathrm{D}$ phase space $\theta, \phi, L$, calculated as described in section 3 . The average reconstruction efficiencies for the data and Monte Carlo samples considering only statistical uncertainties, are

$$
\begin{aligned}
& \epsilon_{\text {data }}^{\text {corr }}=(97.1 \pm 0.1) \% \\
& \epsilon_{\mathrm{MC}}=(97.4 \pm 0.1) \%
\end{aligned}
$$

for data and Monte Carlo, respectively.

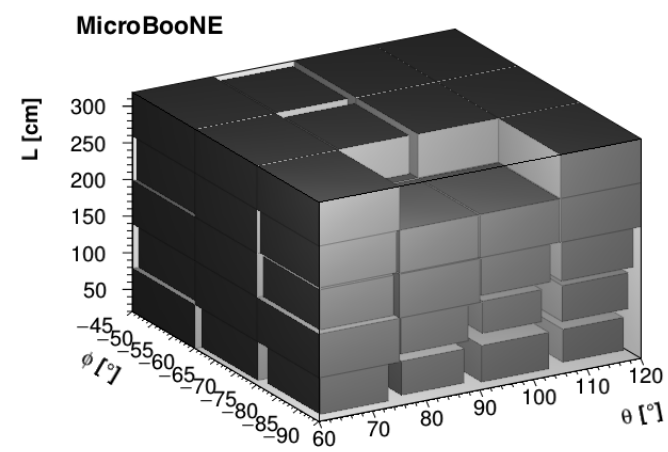

(a) Monte Carlo

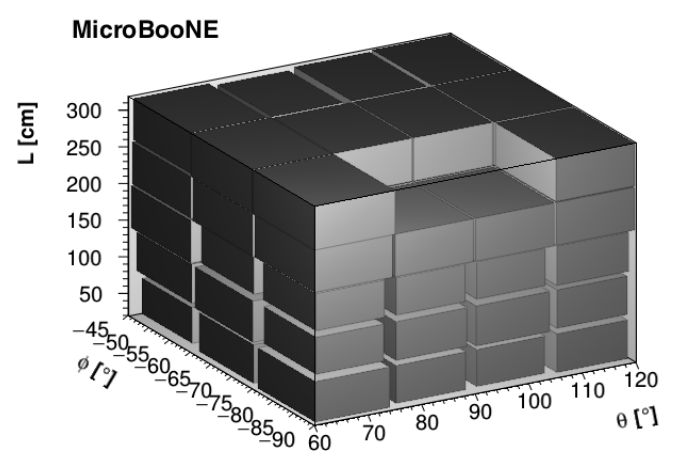

(b) Data

Figure 7. Three-dimensional representation of reconstruction efficiency as a function of the starting angles $\theta, \phi$ and the extrapolated track length $L$ for (a) Monte Carlo and (b) data. The size of the box represents the efficiency. The empty region in the upper part of the plot corresponds to a region of the parameter space not covered by the data sample.

\subsection{Systematic uncertainties}

The measurement of the reconstruction efficiency can be affected by several systematic effects. In particular, the datasets are taken with the MuCS placed in three different geometrical configurations and the MuCS-triggered cosmic muons undergo multiple Coulomb scattering. In this section, the details of all the systematic effects studied are given. 


\subsubsection{Effect of the space-charge effect correction}

The space-charge effect causes a displacement of the ionization tracks. We correct the reconstructed track end points in the TPC vertically to lie on the boundary of the TPC. The error related to this correction is verified to have a negligible effect $(<0.1 \%)$ on the data reconstruction efficiency measurement.

\subsubsection{Effect of the $d_{\max }$ requirement}

As shown in figure 6, the value of $\epsilon_{\text {data }}$ has a small dependence on $d_{\max }$. The difference $0.2 \%$ between the highest and lowest value of $\epsilon_{\text {data }}$ is taken as the systematic uncertainty due to the $d_{\max }$ requirement. This difference could be caused by the multiple Coulomb scattering of the cosmic rays in the box material, which is not included in the MuCS simulation.

\subsubsection{Decay-in-flight or captured muons}

The Monte Carlo statistical uncertainty of the correction factor $D$, as defined in eq. (4.9), is $0.1 \%$ and is taken as the systematic uncertainty related to this correction. Cosmic rays can also be captured or decay inside the detector. Therefore, in these cases, the extrapolated length $L$ will not correspond to the real length of the cosmic-ray path in the TPC.

\subsubsection{Detector non-uniformities}

The presence of potential detector non-uniformities can introduce a systematic uncertainty in the measurement of the reconstruction efficiency. In particular, the presence of noisy or unresponsive wires in specific regions of the detector can lower the reconstruction efficiency. The three different MuCS configurations (shown in figure 3) cover different regions of the TPC, providing information on potential non-uniformities.

To check if these non-uniformities introduce a systematic effect, the significance $\sigma$ of the differences between the data reconstruction efficiencies measured for two different configurations with the following definition is calculated as

$$
\sigma=\frac{\epsilon_{a}-\epsilon_{b}}{\sqrt{\left(\Delta \epsilon_{a}\right)^{2}+\left(\Delta \epsilon_{b}\right)^{2}}}
$$

where $\epsilon_{a}\left(\epsilon_{b}\right)$ is the reconstruction efficiency in the arbitrary $a(b)$ configuration studied and $\Delta \epsilon_{a}\left(\Delta \epsilon_{b}\right)$ is the corresponding statistical uncertainty. This significance is measured for each corresponding $(\theta, \phi, L)$ bin and for each possible combination of central, downstream, and upstream configurations. In the presence of a systematic effect, the standard deviation of the significances distribution would be larger than unity. A Gaussian fit of the distribution gives a standard deviation of $1.54 \pm 0.12$, suggesting that detector non-uniformities are indeed present.

We study cosmic rays corresponding to bins with larger $\sigma$ in more detail and two contributing factors that lead to non-uniformities: regions of the detector with unresponsive wires and highly inclined tracks. The MuCS-extrapolated tracks from the bins with a $\sigma>3$, which drive the broadening of the distribution, show that for the upstream configuration the cosmic rays go through regions with noisy or unresponsive wires in one of the induction planes (figure 8), which are the source of the detector non-uniformities. In addition, the MuCS-extrapolated tracks in these bins 


\section{A. Measurement of cosmic-ray reconstruction efficiencies in the MicroBooNE}

have an orientation as shown in figure 8, implying that the cosmic rays are aligned with the wires of the collection plane, parallel to the $y$ axis. These tracks, therefore, have few hits in two of the three planes, affecting the reconstruction efficiency. Figure 9 shows an event display of a nonreconstructed MuCS cosmic ray going through the region with noisy or unresponsive wires and parallel to the collection plane wires.

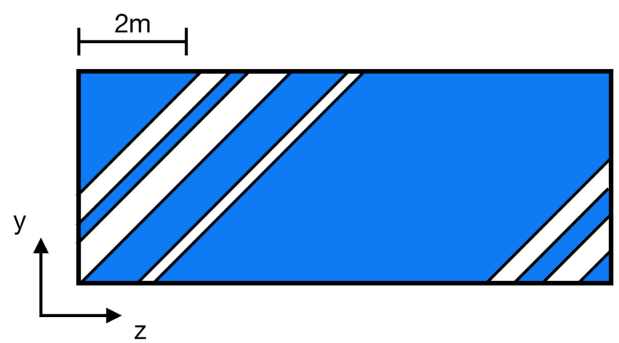

(a) Noisy or unresponsive wires regions

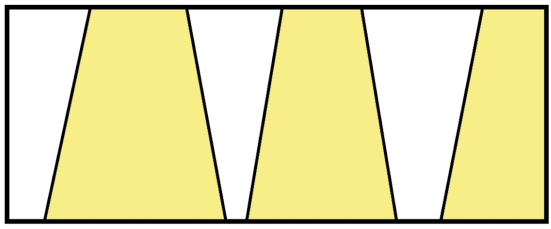

(b) Extrapolated tracks

Figure 8. (a): two-dimensional display of one of the induction planes in the MicroBooNE detector, showing in white the regions with noisy or unresponsive wires. (b): in yellow, the regions of the detector covered by the extrapolated tracks corresponding to bins with $\sigma>3$, as described in the text. As shown in (b), the tracks in the upstream part of the detector (low $z$ ) traverse a region with several noisy or unresponsive wires.

The systematic uncertainty related to the detector non-uniformities for each $\theta, \phi, L$ bin is calculated as the difference between the best reconstruction efficiency of the three configurations and the averaged reconstruction efficiency obtained by merging the three datasets. The systematic uncertainty for the integrated 3D efficiency is $1.1 \%$.

\subsubsection{Energy sampling}

The multiple Coulomb scattering of cosmic muons depends on the energy of the cosmic ray [13]. Thus, low energy cosmic rays scatter more and have a higher probability to be outside the $d_{\max }$ region. They are also more difficult to reconstruct, since their path in the TPC is not a straight line. In the $(\theta, \phi, L)$ bins where the data reconstruction efficiency is measured with low statistics, the MuCS cosmic rays can be distributed in a small region of the energy spectrum [8]. This systematic bias of the reconstruction efficiency, estimated with a dedicated Monte Carlo simulation, is found to be negligible with the present level of statistics $(<0.1 \%)$.

\subsection{Data/Monte Carlo comparison}

The reconstruction efficiencies for the Monte Carlo and data samples are calculated as described in section 4.1.

Figure 10 shows the efficiencies computed for the two-dimensional planes $(\theta, \phi),(\theta, L)$, and $(\phi, L)$, and figure 11 shows the efficiencies computed as a function of $\theta, \phi$, and $L$ individually.

The reconstruction efficiency increases with the expected track length $L$ in the TPC, since longer tracks correspond, in general, to a larger number of hit wires that are easier to reconstruct. The only requirement in the reconstruction efficiency measurement is an extrapolated length in the TPC of $L>20 \mathrm{~cm}$. 
A. Measurement of cosmic-ray reconstruction efficiencies in the MicroBooNE LArTPC using a small external cosmic-ray counter

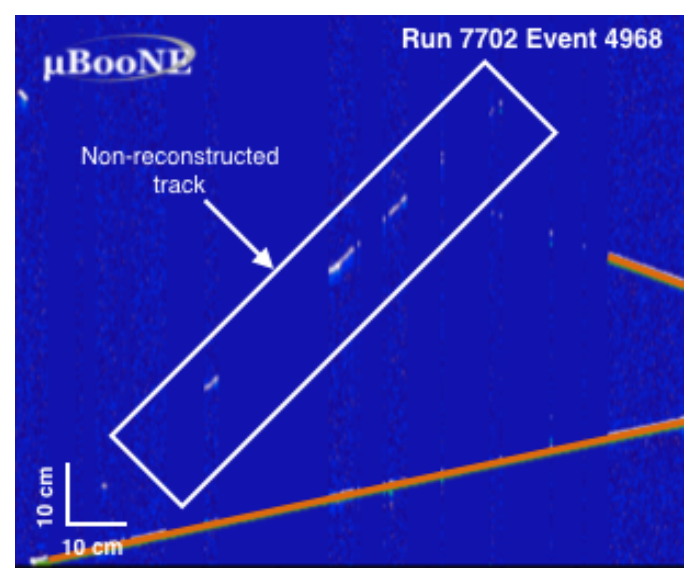

(a) Induction $\left(+60^{\circ}\right)$ plane

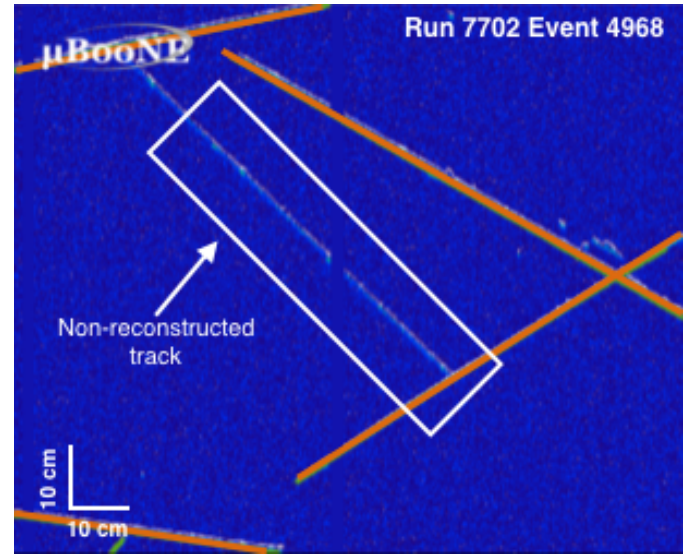

(b) Induction $\left(-60^{\circ}\right)$ plane

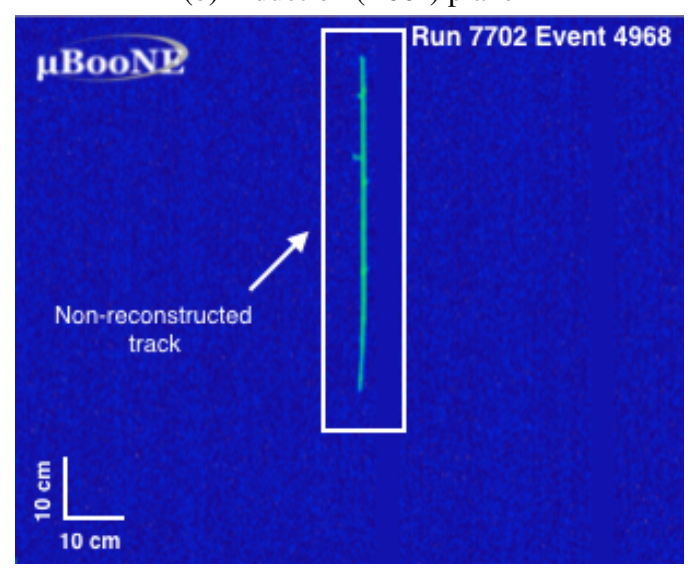

(c) Collection $\left(0^{\circ}\right)$ plane

Figure 9. Event display of a non-reconstructed MuCS cosmic-ray track in the three wire planes, indicated in the white boxes. Orange lines correspond to other TPC reconstructed tracks. Figure 9a shows that the cosmic ray is going through a region with missing or unresponsive wires, while in figure $9 \mathrm{c}$ the cosmic ray is parallel to the collection plane wires. The number of hits was not enough for the algorithm to reconstruct a track. 


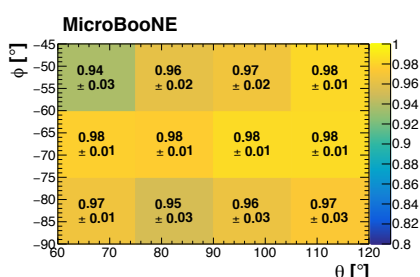

(a) $(\theta, \phi)$-data

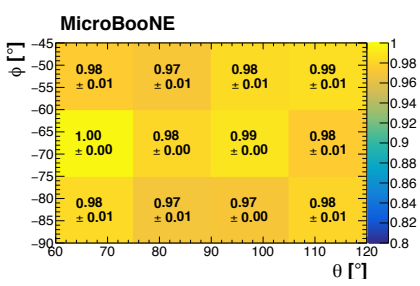

(b) $(\theta, \phi)$-Monte Carlo

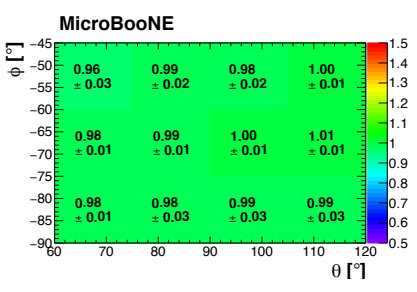

(c) $(\theta, \phi)$-data/Monte Carlo

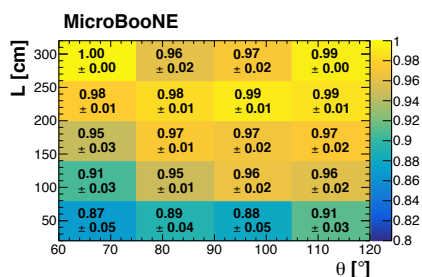

(d) $(\theta, L)$-data

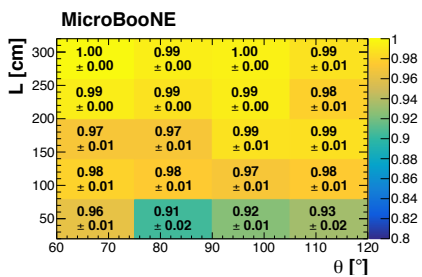

(e) $(\theta, L)$-Monte Carlo

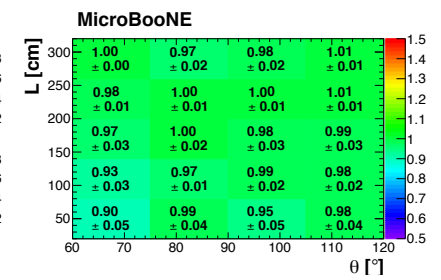

(f) $(\theta, L)$-data/Monte Carlo

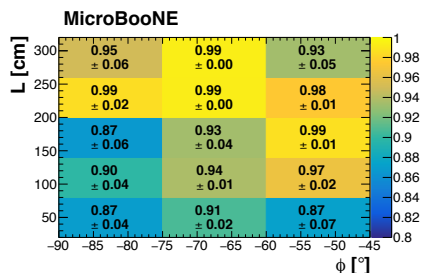

(g) $(\phi, L)$-data

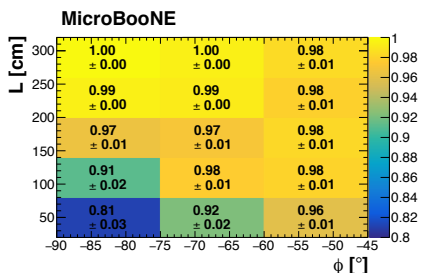

(h) $(\phi, L)$-Monte Carlo

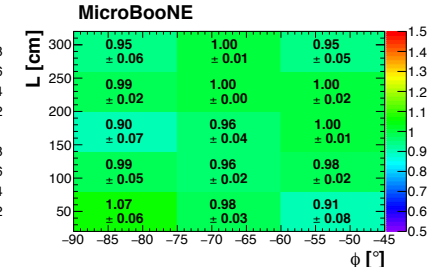

(i) $(\phi, L)$-data/Monte Carlo

Figure 10. Two-dimensional representation of reconstruction efficiencies for data (left), Monte Carlo (center) and their ratio (right). Data uncertainties include systematic effects, while Monte Carlo uncertainties are statistical-only.

The average reconstruction efficiencies for the data and Monte Carlo samples considering the systematic uncertainties, added in quadrature, in the analysis of the data are

$$
\begin{aligned}
\epsilon_{\text {data }}^{\text {corr }} & =(97.1 \pm 0.1 \text { (stat) } \pm 1.4(\text { sys })) \%, \\
\epsilon_{\mathrm{MC}} & =(97.4 \pm 0.1 \text { (stat) }) \%,
\end{aligned}
$$

for data and Monte Carlo, showing good agreement within uncertainties.

\section{Conclusions}

Cosmic muons traversing a LArTPC detector located on the surface can produce a source of backgrounds to the analyses of neutrino interactions. Measuring the reconstruction efficiency of such cosmic rays in the detector is of fundamental importance for the assessment of the detector performance and the suppression of cosmic-ray background.

We present results using data from a small muon counter (the MuCS), placed above the MicroBooNE TPC, to measure the data reconstruction efficiency and compare it with the Monte Carlo reconstruction efficiency. A method to evaluate the number of reconstructed MuCS cosmic rays is studied using a dedicated Monte Carlo simulation. 
A. Measurement of cosmic-ray reconstruction efficiencies in the MicroBooNE LArTPC using a small external cosmic-ray counter

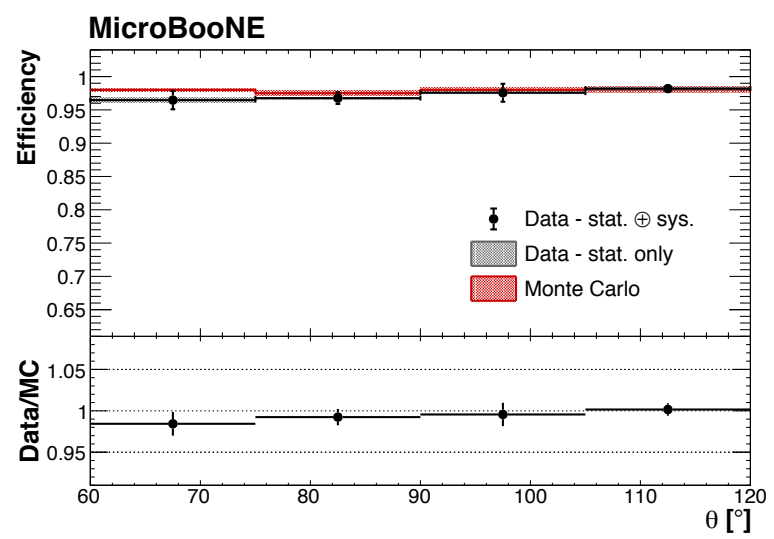

(a) $\theta$

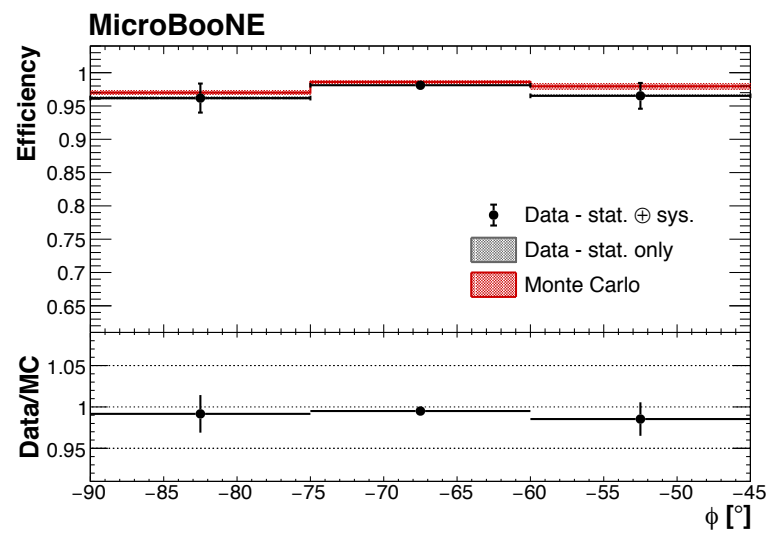

(b) $\phi$

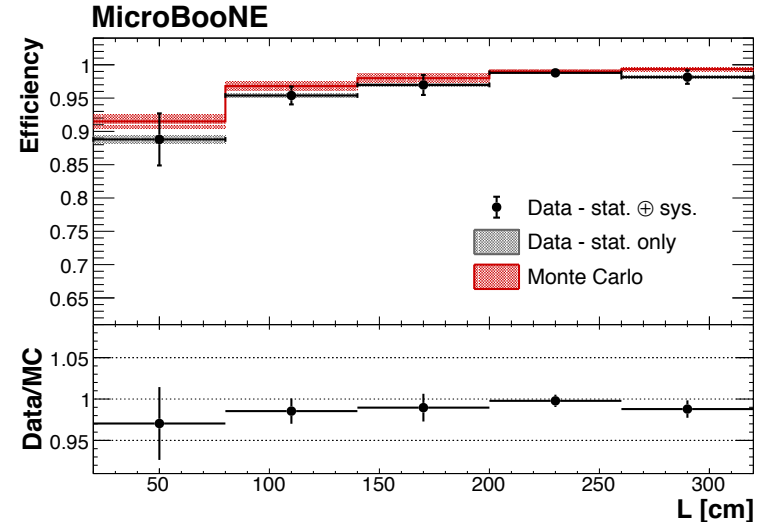

(c) $L$

Figure 11. Monte Carlo (red line) and data (black points) reconstruction efficiency as a function of the starting angles $\theta, \phi$ and the extrapolated track length $L$. Data uncertainty bars include statistical uncertainties and systematic effects, while Monte Carlo uncertainties are statistical-only. 


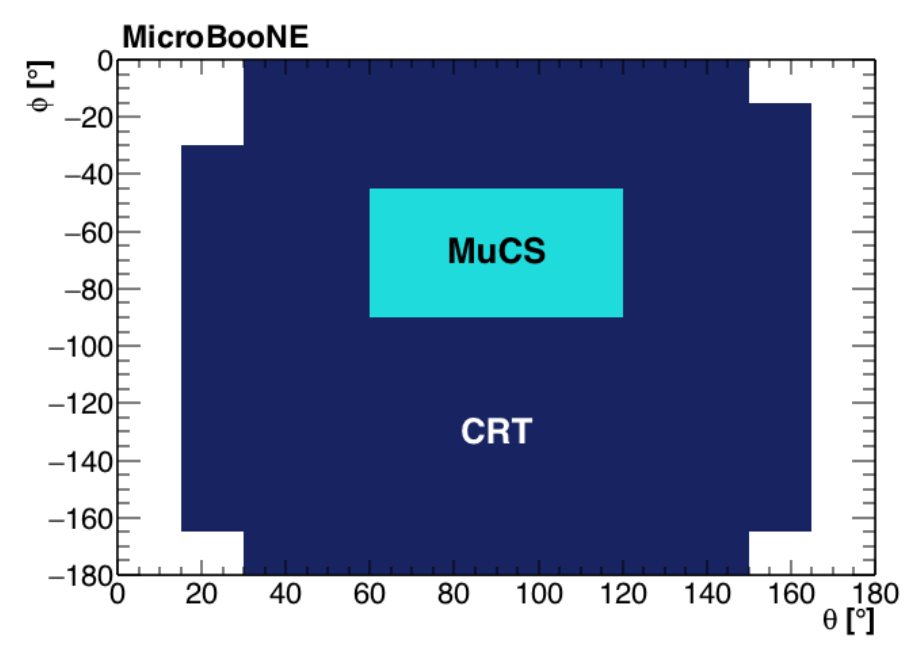

Figure 12. Monte Carlo simulation of the coverage in the $(\theta, \phi)$ plane for both the MuCS (light blue), as presented in this article, and the CRT system (dark blue).

The reconstruction efficiency presented here only assesses whether a track is found and reconstructed. The efficiency here does not quantify the overall quality and accuracy of the track reconstruction in the TPC, such as the correctness of the track length or angle; this will be addressed in a future publication.

The data reconstruction efficiency, calculated by comparing the number of MuCS-triggered events with the number of events with a reconstructed MuCS cosmic ray, is measured as a function of the cosmic-ray starting angles $\theta, \phi$ and the expected length in the TPC, $L$. The overall reconstruction efficiency obtained is $\epsilon_{\text {data }}=(97.1 \pm 0.1$ (stat) \pm 1.4 (sys) $) \%$ and $\epsilon_{\mathrm{MC}}=(97.4 \pm 0.1$ (stat) $) \%$ for data and Monte Carlo, respectively. The two values are consistent within the uncertainties.

We also analyzed systematic uncertainties that affect the data reconstruction efficiency, which amount to $1.4 \%$. The fraction of muons triggering the MuCS that decay or are captured before reaching the TPC is $\approx 1.0 \%$, according to the Monte Carlo simulation described in section 4.2.3. This factor is taken into account in the measurement of the data reconstruction efficiency.

In our analysis, we mention only cosmic-muons. However, the results apply to any minimumionizing particles such as pions. The MuCS setup has a gap of $80 \mathrm{~cm}$ between the two boxes, and our trigger requires a particle to go through both boxes with a clean hit topology. This requirement is not satisfied by photons and neutrons. The fraction of cosmic protons and pions triggering the MuCS and reaching the TPC is, compared to muons, $0.04 \%$ and $0.02 \%$, respectively, and therefore negligible in our analysis.

This article describes a proof of principle method of using an external muon counter to measure the cosmic-ray reconstruction efficiency in a LArTPC. The $(\theta, \phi, L)$ parameter space covered by the MuCS will be significantly expanded using the data coming from a larger cosmic ray tagger system (CRT), installed in March 2017, as illustrated in figure 12. This detector will be able to tag $\approx 80 \%$ of the cosmic rays hitting the TPC and study the presence of non-uniformities in a larger portion of the MicroBooNE detector. The data coming from the CRT will allow the measurement of efficiency-corrected quantities, such as the cosmic-ray flux, and the reconstruction efficiencies will be directly applicable to physics measurements. 
A. Measurement of cosmic-ray reconstruction efficiencies in the MicroBooNE LArTPC using a small external cosmic-ray counter

\section{Acknowledgments}

This material is based upon work supported by the following: the U.S. Department of Energy, Office of Science, Offices of High Energy Physics and Nuclear Physics; the U.S. National Science Foundation; the Swiss National Science Foundation; the Science and Technology Facilities Council of the United Kingdom; and The Royal Society (United Kingdom). Fermilab is operated by Fermi Research Alliance, LLC under Contract No. DE-AC02-07CH11359 with the United States Department of Energy. The Muon Counter Stack and its dedicated DAQ were provided by the Virginia Polytechnic Institute and State University externally to the MicroBooNE collaboration using spare electronics from the Double Chooz experiment provided by Columbia University's Nevis Laboratories.

\section{References}

[1] MicroBooNE collaboration, R. Acciarri et al., Design and Construction of the MicroBooNE Detector, 2017 JINST 12 P02017 [arXiv: 1612 . 05824].

[2] MiniBooNE collaboration, A.A. Aguilar-Arevalo et al., Improved Search for $\bar{v}_{\mu} \rightarrow \bar{v}_{e}$ Oscillations in the MiniBooNE Experiment, Phys. Rev. Lett. 110 (2013) 161801 [arXiv: 1303 . 2588].

[3] DUNE collaboration, R. Acciarri et al., Long-Baseline Neutrino Facility (LBNF) and Deep Underground Neutrino Experiment (DUNE), arXiv: 1601.05471.

[4] M. Auger et al., A Novel Cosmic Ray Tagger System for Liquid Argon TPC Neutrino Detectors, Instruments 1 (2017) 2 [arXiv: 1612.04614].

[5] S.R. Soleti, The Muon Counter System for the MicroBooNE experiment, arXiv: 1604.07858.

[6] J.S. Marshall and M.A. Thomson, The Pandora Software Development Kit for Pattern Recognition, Eur. Phys. J. C 75 (2015) 439 [arXiv: 1506. 05348].

[7] S. Palestini and K. McDonald, Space Charge in Ionization Detectors, accessed 19 October 2017, http://physics.princeton.edu/ mcdonald/examples/spacecharge.pdf.

[8] D. Heck et al., CORSIKA: A Monte Carlo code to simulate extensive air showers, FZKA-6019 (1998).

[9] GEANT4 collaboration, S. Agostinelli et al., GEANT4: A simulation toolkit, Nucl. Instrum. Meth. A 506 (2003) 250.

[10] E.D. Church, LArSoft: A Software Package for Liquid Argon Time Projection Drift Chambers, arXiv: 1311.6774 .

[11] M. Mooney, The MicroBooNE Experiment and the Impact of Space Charge Effects, arXiv: 1511.01563.

[12] MicroBooNE collaboration, R. Acciarri et al., The Pandora multi-algorithm approach to automated pattern recognition of cosmic-ray muon and neutrino events in the MicroBooNE detector, submitted to Eur. Phys. J. C (2017) [arXiv: 1708.03135].

[13] Particle Data Group collaboration, C. Patrignani et al., Review of Particle Physics, Chin. Phys. C 40 (2016) 100001. 


\section{References}

[1] E. Fermi, "Tentativo di una teoria dell'emissione dei raggi beta", Ric. Sci., vol. 4, pp. 491-495, 1933.

[2] W. Pauli, "Dear radioactive ladies and gentlemen", Phys. Today, vol. 31N9, p. 27, 1978.

[3] C. L. Cowan, F. Reines, F. B. Harrison, et al., "Detection of the free neutrino: A Confirmation", Science, vol. 124, pp. 103-104, 1956. DOI: 10.1126/science.124.3212.103.

[4] R. P. Feynman and M. Gell-Mann, "Theory of Fermi interaction", Phys. Rev., vol. 109, pp. 193-198, 1958, [,417(1958)]. DOI: 10.1103/PhysRev.109.193.

[5] M. Goldhaber, L. Grodzins, and A. W. Sunyar, "Helicity of Neutrinos", Phys. Rev., vol. 109, pp. 1015-1017, 1958. DOI: 10.1103/PhysRev.109.1015.

[6] A. de Gouvea, "TASI lectures on neutrino physics", in Physics in $D>=4$. Proceedings, Theoretical Advanced Study Institute in elementary particle physics, TASI 2004, Boulder, USA, June 6-July 2, 2004, 2004, pp. 197-258. arXiv: hep-ph/0411274 [hep-ph].

[7] R. N. Cahn, D. A. Dwyer, S. J. Freedman, et al., "White Paper: Measuring the Neutrino Mass Hierarchy", in Proceedings, 2013 Community Summer Study on the Future of U.S. Particle Physics: Snowmass on the Mississippi (CSS2013): Minneapolis, MN, USA, July 29-August 6, 2013, 2013. arXiv: 1307.5487 [hep-ex]. [Online]. Available: http://www.slac.stanford.edu/econf/ C1307292/docs/submittedArxivFiles/1307.5487.pdf.

[8] G. Danby, J.-M. Gaillard, K. Goulianos, et al., "Observation of high-energy neutrino reactions and the existence of two kinds of neutrinos", Phys. Rev. Lett., vol. 9, pp. 36-44, 1 Jul. 1962. DOI: 10.1103/PhysRevLett.9.36. [Online]. Available: https://link.aps.org/doi/10.1103/PhysRevLett.9.36.

[9] K. Kodama et al., "Observation of tau neutrino interactions", Phys. Lett., vol. B504, pp. 218-224, 2001. DOI: 10.1016/S0370-2693(01)00307-0 arXiv: hep-ex/0012035 [hep-ex].

[10] S. Schael et al., "Precision electroweak measurements on the $Z$ resonance", Phys. Rept., vol. 427, pp. 257-454, 2006. DOI: 10.1016/j.physrep.2005.12.006. arXiv: hep-ex/0509008 [hep-ex].

[11] R. Davis Jr., D. S. Harmer, and K. C. Hoffman, "Search for neutrinos from the sun", Phys. Rev. Lett., vol. 20, pp. 1205-1209, 1968. DOI:

10.1103/PhysRevLett.20.1205 
[12] J. N. Bahcall, M. H. Pinsonneault, and S. Basu, "Solar models: Current epoch and time dependences, neutrinos, and helioseismological properties", Astrophys. J., vol. 555, pp. 990-1012, 2001. DOI: 10.1086/321493, arXiv: astro-ph/0010346 [astro-ph].

[13] K. S. Hirata et al., "Observation of B-8 Solar Neutrinos in the Kamiokande-II Detector", Phys. Rev. Lett., vol. 63, p. 16, 1989. DOI: 10.1103/PhysRevLett.63.16.

[14] D. Abdurashitov et al., "Results from SAGE", Phys. Lett., vol. B328, pp. 234-248, 1994. DOI: 10.1016/0370-2693(94)90454-5

[15] W. Hampel et al., "GALLEX solar neutrino observations: Results for GALLEX IV", Phys. Lett., vol. B447, pp. 127-133, 1999. DOI: 10.1016/S0370-2693(98)01579-2.

[16] Q. R. Ahmad et al., "Direct evidence for neutrino flavor transformation from neutral current interactions in the Sudbury Neutrino Observatory", Phys. Rev. Lett., vol. 89, p. 011301, 2002. DOI: 10.1103/PhysRevLett.89.011301. arXiv: nucl-ex/0204008 [nucl-ex].

[17] L. Wolfenstein, "Neutrino Oscillations in Matter", Phys. Rev., vol. D17, pp. 2369-2374, 1978, [,294(1977)]. DOI: 10.1103/PhysRevD.17.2369.

[18] Y. Fukuda et al., "Evidence for oscillation of atmospheric neutrinos", Phys. Rev. Lett., vol. 81, pp. 1562-1567, 1998. DOI: 10.1103/PhysRevLett.81.1562 arXiv: hep-ex/9807003 [hep-ex].

[19] M. Tanabashi et al., "Review of particle physics", Phys. Rev. D, vol. 98, p. 030 001, 3 Aug. 2018. DOI: 10.1103/PhysRevD.98.030001. [Online]. Available: https://link.aps.org/doi/10.1103/PhysRevD.98.030001

[20] A. Gando et al., "Constraints on $\theta_{13}$ from A Three-Flavor Oscillation Analysis of Reactor Antineutrinos at KamLAND", Phys. Rev., vol. D83, p. 052 002, 2011. DOI: 10.1103/PhysRevD.83.052002, arXiv: 1009.4771 [hep-ex]

[21] I. Esteban, M. C. Gonzalez-Garcia, A. Hernandez-Cabezudo, et al., "Global analysis of three-flavour neutrino oscillations: synergies and tensions in the determination of $\theta_{2} 3, \delta_{C} P$, and the mass ordering", 2018. arXiv: 1811.05487 [hep-ph].

[22] M. Fukugita and T. Yanagida, "Baryogenesis Without Grand Unification", Phys. Lett., vol. B174, pp. 45-47, 1986. DOI: 10.1016/0370-2693(86)91126-3

[23] A. Yu. Smirnov, "Neutrino mass hierarchy and matter effects", PoS, vol. Neutel2013, p. 027, 2014. DOI: 10.22323/1.196.0027. arXiv: 1312.7309 [hep-ph].

[24] N. Aghanim et al., "Planck 2018 results. VI. Cosmological parameters", 2018. arXiv: 1807.06209 [astro-ph.CO].

[25] E. W. Otten and C. Weinheimer, "Neutrino mass limit from tritium beta decay", Rept. Prog. Phys., vol. 71, p. 086 201, 2008. DOI: 10.1088/0034-4885/71/8/086201. arXiv: 0909.2104 [hep-ex].

[26] A. Osipowicz et al., "KATRIN: A Next generation tritium beta decay experiment with sub-eV sensitivity for the electron neutrino mass. Letter of intent", 2001. arXiv: hep-ex/0109033 [hep-ex]. 
[27] C. Arnaboldi et al., "CUORE: A Cryogenic underground observatory for rare events", Nucl. Instrum. Meth., vol. A518, pp. 775-798, 2004. DOI: 10.1016/j.nima.2003.07.067, arXiv: hep-ex/0212053 [hep-ex]

[28] S. Andringa et al., "Current Status and Future Prospects of the SNO+ Experiment", Adv. High Energy Phys., vol. 2016, p. 6194 250, 2016. DOI: 10.1155/2016/6194250, arXiv: 1508.05759 [physics.ins-det]

[29] A. Gando et al., "Search for Majorana Neutrinos near the Inverted Mass Hierarchy Region with KamLAND-Zen", Phys. Rev. Lett., vol. 117, no. 8, p. 082 503, 2016, [Addendum: Phys. Rev. Lett.117,no.10,109903(2016)]. DOI: 10.1103/PhysRevLett.117.109903,10.1103/PhysRevLett.117.082503 arXiv: 1605.02889 [hep-ex]

[30] M. Auger et al., "Search for Neutrinoless Double-Beta Decay in ${ }^{136}$ Xe with EXO-200", Phys. Rev. Lett., vol. 109, p. 032 505, 2012. DOI: 10.1103/PhysRevLett.109.032505, arXiv: 1205.5608 [hep-ex]

[31] V. Alvarez et al., "NEXT-100 Technical Design Report (TDR): Executive Summary", JINST, vol. 7, T06001, 2012. DOI: 10.1088/1748-0221/7/06/T06001 arXiv: 1202.0721 [physics.ins-det].

[32] M. Agostini et al., "Results on Neutrinoless Double- $\beta$ Decay of ${ }^{76}$ Ge from Phase I of the GERDA Experiment", Phys. Rev. Lett., vol. 111, no. 12, p. 122 503, 2013. DOI: 10.1103/PhysRevLett.111.122503, arXiv: 1307.4720 [nucl-ex].

[33] Y. Grossman, "TASI 2002 lectures on neutrinos", in Particle physics and cosmology: The quest for physics beyond the standard model(s). Proceedings, Theoretical Advanced Study Institute, TASI 2002, Boulder, USA, June 3-28, 2002, 2003, pp. 5-48. arXiv: hep-ph/0305245 [hep-ph]

[34] J. A. Formaggio and G. P. Zeller, "From eV to EeV: Neutrino Cross Sections Across Energy Scales", Rev. Mod. Phys., vol. 84, pp. 1307-1341, 2012. DOI: 10.1103/RevModPhys.84.1307, arXiv: 1305.7513 [hep-ex]

[35] C. H. Llewellyn Smith, "Neutrino Reactions at Accelerator Energies", Phys. Rept., vol. 3, pp. 261-379, 1972. DOI: 10.1016/0370-1573(72)90010-5.

[36] R. A. Smith and E. J. Moniz, "Neutrino Reactions on Nuclear Targets", Nucl. Phys., vol. B43, p. 605, 1972, [Erratum: Nucl. Phys.B101,547(1975)]. DOI: 10.1016/0550-3213(75)90612-4,10.1016/0550-3213(72)90040-5.

[37] O. Benhar, N. Farina, H. Nakamura, et al., "Electron- and neutrino-nucleus scattering in the impulse approximation regime", Phys. Rev., vol. D72, p. 053005 , 2005. DOI: 10.1103/PhysRevD.72.053005, arXiv: hep-ph/0506116 [hep-ph]

[38] A. Bodek, H. S. Budd, and M. E. Christy, "Neutrino Quasielastic Scattering on Nuclear Targets: Parametrizing Transverse Enhancement (Meson Exchange Currents)", Eur. Phys. J., vol. C71, p. 1726, 2011. DOI: 10.1140/epjc/s10052-011-1726-y. arXiv: 1106.0340 [hep-ph].

[39] D. Akimov et al., "Observation of Coherent Elastic Neutrino-Nucleus Scattering", Science, vol. 357, no. 6356 , pp. 1123-1126, 2017. DoI: $10.1126 /$ science.aao0990 arXiv: 1708.01294 [nucl-ex]. 
[40] D. Rein and L. M. Sehgal, "Neutrino Excitation of Baryon Resonances and Single Pion Production", Annals Phys., vol. 133, pp. 79-153, 1981. DOI: 10.1016/0003-4916(81)90242-6.

[41] U.-K. Yang and A. Bodek, "Parton distributions, $d / u$, and higher twist effects at high x", Phys. Rev. Lett., vol. 82, pp. 2467-2470, 1999. DOI: 10.1103/PhysRevLett.82.2467, arXiv: hep-ph/9809480 [hep-ph].

[42] T. Golan, C. Juszczak, and J. T. Sobczyk, "Final State Interactions Effects in Neutrino-Nucleus Interactions", Phys. Rev., vol. C86, p. 015 505, 2012. DOI: 10.1103/PhysRevC.86.015505, arXiv: 1202.4197 [nucl-th].

[43] A. Aguilar-Arevalo et al., "Evidence for neutrino oscillations from the observation of anti-neutrino(electron) appearance in a anti-neutrino(muon) beam", Phys. Rev., vol. D64, p. 112 007, 2001. DOI: 10.1103/PhysRevD.64.112007, arXiv: hep-ex/0104049 [hep-ex].

[44] C. Athanassopoulos et al., "Evidence for $\mathrm{nu}(\mathrm{mu}) \rightarrow \mathrm{nu}(\mathrm{e})$ neutrino oscillations from LSND", Phys. Rev. Lett., vol. 81, pp. 1774-1777, 1998. DOI: 10.1103/PhysRevLett.81.1774. arXiv: nucl-ex/9709006 [nucl-ex].

[45] K. Eitel, "Latest results of the KARMEN2 experiment", Nucl. Phys. Proc. Suppl., vol. 91, pp. 191-197, 2001, [,191(2000)]. DOI: 10.1016/S0920-5632(00) 00940-3 arXiv: hep-ex/0008002 [hep-ex].

[46] A.A. Aguilar-Arevalo et al., "The Neutrino Flux prediction at MiniBooNE", Phys. Rev., vol. D79, p. 072 002, 2009. DOI: 10.1103/PhysRevD.79.072002 arXiv: 0806.1449 [hep-ex].

[47] T. Katori, "Cross section analyses in MiniBooNE and SciBooNE experiments", AIP Conf. Proc., vol. 1663, p. 020 001, 2015. DOI: 10.1063/1.4919461. arXiv: 1304.5325 [hep-ex]

[48] A. A. Aguilar-Arevalo et al., "Significant Excess of ElectronLike Events in the MiniBooNE Short-Baseline Neutrino Experiment", Phys. Rev. Lett., vol. 121, p. 221801,2018 . DOI: 10.1103/PhysRevLett.121.221801. arXiv: 1805.12028 [hep-ex].

[49] G. S. Karagiorgi, "Searches for New Physics at MiniBooNE: Sterile Neutrinos and Mixing Freedom", PhD thesis, MIT, 2010. DOI: 10.2172/1000269. [Online]. Available: http://lss.fnal.gov/cgi-bin/find_paper.pl?thesis-2010-39

[50] N. Agafonova et al., "Final results of the search for $\nu_{\mu} \rightarrow \nu_{e}$ oscillations with the OPERA detector in the CNGS beam", JHEP, vol. 06, p. 151, 2018. DOI: 10.1007/JHEP06 (2018) 151, arXiv: 1803.11400 [hep-ex]

[51] C. Giunti and M. Laveder, "Statistical Significance of the Gallium Anomaly", Phys. Rev., vol. C83, p. 065 504, 2011. DOI: 10.1103/PhysRevC.83.065504 arXiv: 1006.3244 [hep-ph].

[52] J. N. Abdurashitov et al., "Measurement of the solar neutrino capture rate with gallium metal", Phys. Rev., vol. C60, p. 055801,1999 . DOI: 10.1103/PhysRevC.60.055801, arXiv: astro-ph/9907113 [astro-ph].

[53] T. A. Mueller et al., "Improved Predictions of Reactor Antineutrino Spectra", Phys. Rev., vol. C83, p. 054 615, 2011. DOI: 10.1103/PhysRevC.83.054615. arXiv: 1101.2663 [hep-ex]. 
[54] F. P. An et al., "Measurement of the Reactor Antineutrino Flux and Spectrum at Daya Bay", Phys. Rev. Lett., vol. 116, no. 6, p. 061 801, 2016, [Erratum: Phys. Rev. Lett.118,no.9,099902(2017)]. DOI: 10.1103/PhysRevLett.116.061801,10.1103/PhysRevLett.118.099902, arXiv: 1508.04233 [hep-ex].

[55] F. P. An et al., "Evolution of the Reactor Antineutrino Flux and Spectrum at Daya Bay", Phys. Rev. Lett., vol. 118, no. 25, p. 251801 , 2017. DoI: 10.1103/PhysRevLett.118.251801. arXiv: 1704.01082 [hep-ex]

[56] P. Huber, "NEOS Data and the Origin of the $5 \mathrm{MeV}$ Bump in the Reactor Antineutrino Spectrum", Phys. Rev. Lett., vol. 118, no. 4, p. 042 502, 2017. DOI: 10.1103/PhysRevLett.118.042502, arXiv: 1609.03910 [hep-ph].

[57] J. Ashenfelter et al., "The PROSPECT Physics Program", J. Phys., vol. G43, no. 11, p. 113 001, 2016. DOI: 10.1088/0954-3899/43/11/113001. arXiv: 1512.02202 [physics.ins-det].

[58] Y. Abreu et al., "A novel segmented-scintillator antineutrino detector", JINST, vol. 12, no. 04, P04024, 2017. DOI: 10.1088/1748-0221/12/04/P04024 arXiv: 1703.01683 [physics.ins-det].

[59] H. Almazán et al., "Sterile Neutrino Constraints from the STEREO Experiment with 66 Days of Reactor-On Data", Phys. Rev. Lett., vol. 121, no. 16, p. 161801 , 2018. DOI: 10.1103/PhysRevLett.121.161801, arXiv: 1806.02096 [hep-ex].

[60] P. Adamson et al., "Limits on Active to Sterile Neutrino Oscillations from Disappearance Searches in the MINOS, Daya Bay, and Bugey-3 Experiments", Phys. Rev. Lett., vol. 117, no. 15, p. 151 801, 2016, [Addendum: Phys. Rev. Lett.117,no.20,209901(2016)]. DOI: 10.1103/PhysRevLett.117.151801,10.1103/PhysRevLett.117.209901. arXiv: 1607.01177 [hep-ex].

[61] M. G. Aartsen et al., "Searches for Sterile Neutrinos with the IceCube Detector", Phys. Rev. Lett., vol. 117, no. 7, p. 071801,2016 . DOI: 10.1103/PhysRevLett.117.071801. arXiv: 1605.01990 [hep-ex]

[62] M. Dentler, Á. Hernández-Cabezudo, J. Kopp, et al., "Updated Global Analysis of Neutrino Oscillations in the Presence of eV-Scale Sterile Neutrinos", JHEP, vol. 08, p. 010, 2018. DOI: 10.1007/JHEP08(2018)010. arXiv: 1803.10661 [hep-ph].

[63] J. M. Conrad, C. M. Ignarra, G. Karagiorgi, et al., "Sterile Neutrino Fits to Short Baseline Neutrino Oscillation Measurements", Adv. High Energy Phys., vol. 2013, p. 163897,2013 . DOI: 10.1155/2013/163897, arXiv: 1207.4765 [hep-ex]

[64] A. Kostelecky and M. Mewes, "Neutrinos with Lorentz-violating operators of arbitrary dimension", Phys. Rev., vol. D85, p. 096 005, 2012. DOI: 10.1103/PhysRevD.85.096005, arXiv: 1112.6395 [hep-ph]

[65] J. Asaadi, E. Church, R. Guenette, et al., "New light Higgs boson and short-baseline neutrino anomalies", Phys. Rev., vol. D97, no. 7, p. 075 021, 2018. DOI: 10.1103/PhysRevD.97.075021, arXiv: 1712.08019 [hep-ph].

[66] J. R. Jordan, Y. Kahn, G. Krnjaic, et al., "Severe Constraints on New Physics Explanations of the MiniBooNE Excess", 2018. arXiv: 1810.07185 [hep-ph]. 
[67] R. Acciarri et al., "First Observation of Low Energy Electron Neutrinos in a Liquid Argon Time Projection Chamber", Phys. Rev., vol. D95, no. 7, p. 072 005, 2017. DOI: 10.1103/PhysRevD.95.072005 arXiv: 1610.04102 [hep-ex].

[68] A. A. Aguilar-Arevalo et al., "First Observation of Coherent $\pi^{0}$ Production in Neutrino Nucleus Interactions with $E_{\nu}<2 \mathrm{GeV",} \mathrm{Phys.} \mathrm{Lett.,} \mathrm{vol.} \mathrm{B664,}$ pp. 41-46, 2008. DOI: 10.1016/j.physletb.2008.05.006 arXiv: 0803.3423 [hep-ex].

[69] K. Hiraide et al., "Search for Charged Current Coherent Pion Production on Carbon in a Few-GeV Neutrino Beam", Phys. Rev., vol. D78, p. 112 004, 2008. DOI: 10.1103/PhysRevD.78.112004, arXiv: 0811.0369 [hep-ex],

[70] H. Tanaka, "K2K coherent pion production in SciBar", Nucl. Phys. Proc. Suppl., vol. 159, pp. 38-43, 2006, [,38(2006)]. DOI: $10.1016 / \mathrm{j}$.nuclphysbps.2006.08.075.

[71] R. Acciarri et al., "Long-Baseline Neutrino Facility (LBNF) and Deep Underground Neutrino Experiment (DUNE)", 2016. arXiv: 1601.02984 [physics.ins-det].

[72] K. Abe et al., "Search for proton decay via $p \rightarrow \nu K^{+}$using 260 kiloton·year data of Super-Kamiokande", Phys. Rev., vol. D90, no. 7, p. 072 005, 2014. DOI: 10.1103/PhysRevD.90.072005, arXiv: 1408.1195 [hep-ex]

[73] P. Antonioli et al., "SNEWS: The Supernova Early Warning System", New J. Phys., vol. 6, p. 114, 2004. DOI: 10.1088/1367-2630/6/1/114. arXiv: astro-ph/0406214 [astro-ph].

[74] S. Amerio et al., "Design, construction and tests of the ICARUS T600 detector", Nucl. Instrum. Meth., vol. A527, pp. 329-410, 2004. DOI: 10.1016/j.nima.2004.02.044.

[75] M. Antonello et al., "Search for anomalies in the $\nu_{e}$ appearance from a $\nu_{\mu}$ beam", Eur. Phys. J., vol. C73, p. 2599, 2013. DOI: 10.1140/epjc/s10052-013-2599-z. arXiv: 1307.4699 [hep-ex].

[76] C. Anderson et al., "The ArgoNeuT Detector in the NuMI Low-Energy beam line at Fermilab", JINST, vol. 7, P10019, 2012. DOI: 10.1088/1748-0221/7/10/P10019, arXiv: 1205.6747 [physics.ins-det]

[77] W. J. Willis and V. Radeka, "Liquid Argon Ionization Chambers as Total Absorption Detectors", Nucl. Instrum. Meth., vol. 120, pp. 221-236, 1974. DOI: 10.1016/0029-554X(74)90039-1

[78] C. Rubbia, "The Liquid Argon Time Projection Chamber: A New Concept for Neutrino Detectors", 1977.

[79] C. Rubbia, "The ICARUS Liquid Argon TPC: A neutrino 'bubble chamber' after Gargamelle", J. Phys. Conf. Ser., vol. 308, p. 012 002, 2011. DOI: 10.1088/1742-6596/308/1/012002.

[80] S. Amoruso et al., "Study of electron recombination in liquid argon with the ICARUS TPC", Nucl. Instrum. Meth., vol. A523, pp. 275-286, 2004. DOI: 10.1016/j.nima.2003.11.423. 
[81] J. B. Birks, "Scintillations from Organic Crystals: Specific Fluorescence and Relative Response to Different Radiations", Proc. Phys. Soc., vol. A64, pp. 874-877, 1951. DOI: $10.1088 / 0370-1298 / 64 / 10 / 303$

[82] J. Thomas and D. A. Imel, "Recombination of electron-ion pairs in liquid argon and liquid xenon", Phys. Rev., vol. A36, pp. 614-616, 1987. DOI: 10.1103/PhysRevA.36.614.

[83] R. Acciarri et al., "Study of Space Charge Effects in MicroBooNE", online: MICROBOONE-NOTE-1018-PUB

[84] V. Meddage, "Electron Attenuation Measurement using Cosmic Ray Muons at the MicroBooNE LArTPC", in Proceedings, Meeting of the APS Division of Particles and Fields (DPF 2017): Fermilab, Batavia, Illinois, USA, July 31 - August 4, 2017, 2017. arXiv: 1710.00396 [physics.ins-det]. [Online]. Available: http://lss.fnal.gov/archive/2017/conf/fermilab-conf-17-517-e.pdf.

[85] M. Antonello et al., "A Proposal for a Three Detector Short-Baseline Neutrino Oscillation Program in the Fermilab Booster Neutrino Beam", 2015. arXiv: 1503.01520 [physics.ins-det].

[86] A. A. Aguilar-Arevalo et al., "The MiniBooNE Detector", Nucl. Instrum. Meth., vol. A599, pp. 28-46, 2009. DOI: 10.1016/j.nima.2008.10.028 arXiv: 0806.4201 [hep-ex]

[87] M. G. Catanesi et al., "Measurement of the production cross-section of positive pions in p-Al collisions at 12.9-GeV/c", Nucl. Phys., vol. B732, pp. 1-45, 2006. DOI: $10.1016 / j \cdot n u c l$ physb.2005.10.016. arXiv: hep-ex/0510039 [hep-ex].

[88] G. Cheng et al., "Measurement of $K^{+}$production cross section by $8 \mathrm{GeV}$ protons using high energy neutrino interactions in the SciBooNE detector", Phys. Rev., vol. D84, p. 012 009, 2011. DOI: 10.1103/PhysRevD.84.012009, arXiv: 1105.2871 [hep-ex]

[89] R. Brun, F. Bruyant, F. Carminati, et al., "GEANT Detector Description and Simulation Tool", 1994. DOI: 10.17181/CERN.MUHF.DMJ1

[90] R. Acciarri et al., "Design and Construction of the MicroBooNE Detector", JINST, vol. 12, no. 02, P02017, 2017. DOI: 10.1088/1748-0221/12/02/P02017. arXiv: 1612.05824 [physics.ins-det].

[91] H. Chen, K. Chen, G. De Geronimo, et al., "Readout electronics for the MicroBooNE LAr TPC, with CMOS front end at 89K", JINST, vol. 7, p. C12004, 2012. DOI: $10.1088 / 1748-0221 / 7 / 12 / \mathrm{C} 12004$

[92] R. Acciarri et al., "Measurement of cosmic-ray reconstruction efficiencies in the MicroBooNE LArTPC using a small external cosmic-ray counter", JINST, vol. 12, no. 12, P12030, 2017. DOI: $10.1088 / 1748-0221 / 12 / 12 / \mathrm{P} 12030$ arXiv: 1707.09903 [hep-ex].

[93] M. Auger et al., "A Novel Cosmic Ray Tagger System for Liquid Argon TPC Neutrino Detectors", Instruments, vol. 1, no. 1, p. 2, 2017. DOI: 10.3390/instruments1010002, arXiv: 1612.04614 [physics.ins-det].

[94] R. Acciarri et al., "Noise Characterization and Filtering in the MicroBooNE Liquid Argon TPC", JINST, vol. 12, no. 08, P08003, 2017. DOI: 10.1088/1748-0221/12/08/P08003 arXiv: 1705.07341 [physics.ins-det] 
[95] C. Adams et al., "Ionization electron signal processing in single phase LArTPCs. Part I. Algorithm Description and quantitative evaluation with MicroBooNE simulation", JINST, vol. 13, no. 07, P07006, 2018. DOI: 10.1088/1748-0221/13/07/P07006, arXiv: 1802.08709 [physics.ins-det].

[96] J. S. Marshall and M. A. Thomson, "The Pandora Software Development Kit for Pattern Recognition", Eur. Phys. J., vol. C75, no. 9, p. 439, 2015. DOI: 10.1140/epjc/s10052-015-3659-3 arXiv: 1506.05348 [physics.data-an]

[97] R. Acciarri et al., "The Pandora multi-algorithm approach to automated pattern recognition of cosmic-ray muon and neutrino events in the MicroBooNE detector", Eur. Phys. J., vol. C78, no. 1, p. 82, 2018. DOI: 10.1140/epjc/s10052-017-5481-6, arXiv: 1708.03135 [hep-ex]

[98] D. Perevalov and R. Tayloe, "Measurement of Neutrino-Nucleon Neutral Current Elastic Scattering in MiniBooNE", AIP Conf. Proc., vol. 1189, pp. 175-180, 2009. DOI: 10.1063/1.3274151, arXiv: 0909.4617 [hep-ex].

[99] C. Andreopoulos et al., "The GENIE Neutrino Monte Carlo Generator", Nucl. Instrum. Meth., vol. A614, pp. 87-104, 2010. DOI: 10.1016/j.nima.2009.12.009 arXiv: 0905.2517 [hep-ph].

[100] D. Heck, J. Knapp, J. N. Capdevielle, et al., "CORSIKA: A Monte Carlo code to simulate extensive air showers", 1998.

[101] E. D. Church, "LArSoft: A Software Package for Liquid Argon Time Projection Drift Chambers", 2013. arXiv: 1311.6774 [physics.ins-det].

[102] R. Acciarri et al., "First Muon-Neutrino Charged-Current Inclusive Differential Cross Section Measurement for MicroBooNE Run 1 Data", online: MICROBOONE-NOTE-1045-PUB.

[103] P. Abratenko et al., "Determination of muon momentum in the MicroBooNE LArTPC using an improved model of multiple Coulomb scattering", JINST, vol. 12 , no. 10 , P10010, 2017. DOI: 10.1088/1748-0221/12/10/P10010 arXiv: 1703.06187 [physics.ins-det].

[104] M. Paterno, "Calculating efficiencies and their uncertainties", 2004. DOI: $10.2172 / 15017262$.

[105] C. Adams et al., "Ionization electron signal processing in single phase LArTPCs. Part II. Data/simulation comparison and performance in MicroBooNE", JINST, vol. 13, no. 07, P07007, 2018. DOI: 10.1088/1748-0221/13/07/P07007, arXiv: 1804.02583 [physics.ins-det].

[106] R. Acciarri et al., "Michel Electron Reconstruction Using Cosmic-Ray Data from the MicroBooNE LArTPC", JINST, vol. 12, no. 09, P09014, 2017. DOI: 10.1088/1748-0221/12/09/P09014 arXiv: 1704.02927 [physics.ins-det]

[107] E. Shibamura, A. Hitachi, T. Doke, et al., "Drift velocities of electrons, saturation characteristics of ionization and $\mathrm{W}$-values for conversion electrons in liquid argon, liquid argon-gas mixtures and liquid xenon", Nucl. Instrum. Meth., vol. 131, pp. 249-258, 1975. DOI: 10.1016/0029-554X (75)90327-4.

[108] R. Acciarri et al., "A study of electron recombination using highly ionizing particles in the ArgoNeuT Liquid Argon TPC", JINST, vol. 8, P08005, 2013. DOI: 10.1088/1748-0221/8/08/P08005. arXiv: 1306.1712 [physics.ins-det]. 
[109] S. Das, "A simple alternative to the Crystal Ball function", 2016. arXiv: 1603.08591 [hep-ex].

[110] C. W. Fabjan and F. Gianotti, "Calorimetry for particle physics", Rev. Mod. Phys., vol. 75, pp. 1243-1286, 2003. DOI: 10.1103/RevModPhys.75.1243.

[111] M. Berger et al., "ESTAR, PSTAR, and ASTAR: Computer Programs for Calculating Stopping-Power and Range Tables for Electrons, Protons, and Helium Ions (version 1.2.3)", National Institute of Standards and Technology, Gaithersburg, MD, 2005. online: http://physics.nist.gov/Star.

[112] L. Landau, "On the energy loss of fast particles by ionization", J. Phys.(USSR), vol. 8, pp. 201-205, 1944.

[113] D. Caratelli, "Study of Electromagnetic Interactions in the MicroBooNE Liquid Argon Time Projection Chamber", PhD thesis, Columbia U., 2018. DOI: 10.2172/1420402. [Online]. Available: http: //lss.fnal.gov/archive/thesis/2000/fermilab-thesis-2018-02.pdf

[114] D. C. Swift and J. M. McNaney, "Approximate, analytic solutions of the Bethe equation for charged particle range", 2008. arXiv: 0901.4145 [cond-mat.other]

[115] F. J. Massey Jr, "The kolmogorov-smirnov test for goodness of fit", Journal of the American statistical Association, vol. 46, no. 253, pp. 68-78, 1951.

[116] H.-J. Yang, B. P. Roe, and J. Zhu, "Studies of boosted decision trees for MiniBooNE particle identification", Nucl. Instrum. Meth., vol. A555, pp. 370-385, 2005. DOI: $10.1016 / j$.nima.2005.09.022 arXiv: physics/0508045 [physics].

[117] V. M. Abazov et al., "Evidence for production of single top quarks and first direct measurement of |Vtb|", Phys. Rev. Lett., vol. 98, p. 181802, 2007. DOI: 10.1103/PhysRevLett.98.181802, arXiv: hep-ex/0612052 [hep-ex].

[118] Y. Coadou, "Boosted Decision Trees and Applications", EPJ Web Conf., vol. 55, p. 02 004, 2013. DOI: 10.1051/epjconf/20135502004.

[119] Y. Freund and R. Schapire, "A short introduction to boosting", Journal-Japanese Society For Artificial Intelligence, vol. 14, no. 771-780, p. 1612, 1999.

[120] A. Hocker et al., "TMVA - Toolkit for Multivariate Data Analysis", 2007. arXiv: physics/0703039 [physics.data-an]

[121] T. Katori, "Meson Exchange Current (MEC) Models in Neutrino Interaction Generators", AIP Conf. Proc., vol. 1663, p. 030 001, 2015. DOI: 10.1063/1.4919465, arXiv: 1304.6014 [nucl-th].

[122] P. Adamson et al., "The NuMI Neutrino Beam", Nucl. Instrum. Meth., vol. A806, pp. 279-306, 2016. DOI: $10.1016 / \mathrm{j}$.nima.2015.08.063 arXiv: 1507.06690 [physics.acc-ph].

[123] G. Cowan, K. Cranmer, E. Gross, et al., "Asymptotic formulae for likelihood-based tests of new physics", Eur. Phys. J., vol. C71, p. 1554, 2011, [Erratum: Eur. Phys. J.C73,2501(2013)]. DOI: 10.1140/epjc/s10052-011-1554-0,10.1140/epjc/s10052-013-2501-z arXiv: 1007.1727 [physics.data-an].

[124] C. Andreopoulos, C. Barry, S. Dytman, et al., "The GENIE Neutrino Monte Carlo Generator: Physics and User Manual", 2015. arXiv: 1510.05494 [hep-ph] 
[125] M. G. Catanesi et al., "Measurement of the production cross-section of positive pions in the collision of $8.9-\mathrm{GeV} / \mathrm{c}$ protons on beryllium", Eur. Phys. J., vol. C52, pp. 29-53, 2007. DOI: $10.1140 / \mathrm{epjc} / \mathrm{s} 10052-007-0382-8$, arXiv: hep-ex/0702024 [hep-ex].

[126] J. R. Sanford and C. L. Wang, "EMPIRICAL FORMULAS FOR PARTICLE PRODUCTION IN P - Be COLLISION BETWEEN 10-GeV/c AND 35-GeV/c", 1967.

[127] J. Nieves, I. Ruiz Simo, and M. J. Vicente Vacas, "Inclusive Charged-Current Neutrino-Nucleus Reactions", Phys. Rev., vol. C83, p. 045 501, 2011. DOI: 10.1103/PhysRevC.83.045501, arXiv: 1102.2777 [hep-ph].

[128] R. Acciarri et al., "MicroBooNE low-energy excess signal prediction from unfolding MiniBooNE Monte-Carlo and data", online: MICROBOONE-NOTE-1043-PUB.

[129] A. A. Aguilar-Arevalo et al., "A Search for electron neutrino appearance at the $\Delta m^{2} \sim 1 \mathrm{eV}^{2}$ scale", Phys. Rev. Lett., vol. 98, p. 231801, 2007. DOI: 10.1103/PhysRevLett.98.231801, arXiv: 0704.1500 [hep-ex].

[130] M. Blennow, P. Coloma, P. Huber, et al., "Quantifying the sensitivity of oscillation experiments to the neutrino mass ordering", JHEP, vol. 03, p. 028, 2014. DOI: 10.1007/JHEP03(2014)028, arXiv: 1311.1822 [hep-ph]

[131] R. Acciarri et al., "The MicroBooNE Search for Single Photon Events", online: MICROBOONE-NOTE-1041-PUB.

[132] C. Adams et al., "LAr1-ND: Testing Neutrino Anomalies with Multiple LArTPC Detectors at Fermilab", in Proceedings, 2013 Community Summer Study on the Future of U.S. Particle Physics: Snowmass on the Mississippi (CSS2013): Minneapolis, MN, USA, July 29-August 6, 2013, 2013. arXiv: 1309.7987 [physics.ins-det]. [Online]. Available: http://www.slac.stanford.edu/ econf/C1307292/docs/submittedArxivFiles/1309.7987.pdf

[133] F. Varanini, "ICARUS detector: present and future", EPJ Web Conf., vol. 164, p. 07 017, 2017. DOI: 10.1051/epjconf/201716407017.

[134] R. Acciarri et al., "Long-Baseline Neutrino Facility (LBNF) and Deep Underground Neutrino Experiment (DUNE)", 2015. arXiv: 1512.06148 [physics.ins-det].

[135] R. Gandhi, B. Kayser, M. Masud, et al., "The impact of sterile neutrinos on CP measurements at long baselines", JHEP, vol. 11, p. 039, 2015. DOI:

10.1007/JHEP11(2015) 039, arXiv: 1508.06275 [hep-ph] 